\title{
Perception in a Variable but Structured World: The Case of Speech Perception by
}

Dave F. Kleinschmidt

Submitted in Partial Fulfillment of the

Requirements for the Degree

Doctor of Philosophy

Supervised by

Professor T. Florian Jaeger

Department of Brain and Cognitive Sciences

Arts, Sciences and Engineering

School of Arts and Sciences

University of Rochester

Rochester, New York 
For Dad and Dave. 


\section{Biographical Sketch}

The author was born in Boston, Massachusetts on March 19th, 1986. He attended Williams College from 2005 to 2009, and graduated summa cum laude with a Bachelor of Arts degree in Mathematics, and a concentration in Cognitive Science (highest honors). From the fall of 2009 until the fall of 2010, he served as a Baggett Fellow at the University of Maryland Department of Linguistics. In the fall of 2010, he began his graduate studies at the University of Rochester in the Department of Brain and Cognitive Sciences, where he was advised by Professor T. Florian Jaeger. He was awarded a Master of Arts degree in Brain and Cognitive Sciences from the University of Rochester in 2013. He was awarded a Sproull Fellowship from the University of Rochester in 2010.

The following manuscripts published or under review, have resulted from the author's work at the University of Rochester:

- Croft, W., Bhattacharya, T., Kleinschmidt, D. F., Smith, D. E., \& Jaeger, T. F. (2011). Greenbergian universals, diachrony, and statistical analyses. Linguistic Typology, 15(2), 433-453. doi:10.1515/LITY.2011.029

- Kleinschmidt, D. F. \& Jaeger, T. F. (2011). A Bayesian belief updating model of phonetic recalibration and selective adaptation. In Proceedings of the 2nd acl workshop on cognitive modeling and computational linguistics. Stroudsburg, PA: Association for Computational Linguistics 
- Kleinschmidt, D. F. \& Jaeger, T. F. (2012). A continuum of phonetic adaptation: Evaluating an incremental belief-updating model of recalibration and selective adaptation. In N. Miyake, D. Peebles, \& R. P. Cooper (Eds.), Proceedings of the 34th annual conference of the cognitive science society (pp. 605-10). Austin, TX: Cognitive Science Society

- Kleinschmidt, D. F., Fine, A. B., \& Jaeger, T. F. (2012). A belief-updating model of adaptation and cue combination in syntactic comprehension. In N. Miyake, D. Peebles, \& R. P. Cooper (Eds.), Proceedings of the 34th annual conference of the cognitive science society (pp. 599-604). Austin, TX: Cognitive Science Society

- Salverda, A. P., Kleinschmidt, D. F., \& Tanenhaus, M. K. (2014). Immediate effects of anticipatory coarticulation in spoken-word recognition. Journal of Memory and Language, 71(1), 145-163. doi:10.1016/j.jml.2013.11.002

- Zaki, S. R. \& Kleinschmidt, D. F. (2014). Procedural memory effects in categorization: Evidence for multiple systems or task complexity? Memory E cognition, 42(3), 508-24. doi:10.3758/s13421-013-0375-9

- Kleinschmidt, D. F. \& Jaeger, T. F. (2015). Robust speech perception: Recognize the familiar, generalize to the similar, and adapt to the novel. Psychological Review, 122(2), 148-203. doi:10.1037/a0038695

- Kleinschmidt, D. F., Raizada, R., \& Jaeger, T. F. (2015). Supervised and unsupervised learning in phonetic adaptation. In R. Dale, C. Jennings, P. Maglio, T. Matlock, D. Noelle, A. Warlaumont, \& J. Yoshimi (Eds.), Proceedings of the 37th annual conference of the cognitive science society. Austin, TX: Cognitive Science Society

- Kleinschmidt, D. F. \& Jaeger, T. F. (2016a). Re-examining selective adaptation: Fatiguing feature detectors, or distributional learning? Psychonomic 
Bulletin \& Review, 23(3), 678-691. doi:10.3758/s13423-015-0943-z

- Kleinschmidt, D. F. \& Jaeger, T. F. (2016b). What do you expect from an unfamiliar talker? In J. Trueswell, A. Papafragou, D. Grodner, \& D. Mirman (Eds.), Proceedings of the 38th annual meeting of the cognitive science society. Austin, TX: Cognitive Science Society

- Pajak, B., Fine, A. B., Kleinschmidt, D. F., \& Jaeger, T. F. (2016). Learning Additional Languages as Hierarchical Probabilistic Inference: Insights From First Language Processing. Language Learning, e-pub ahea. doi:10. 1111/lang.12168

- Weatherholtz, K., Seifeldin, M., Kleinschmidt, D. F., Kurumada, C., \& Jaeger, T. F. (2016). Speech perception as probabilistic inference under uncertainty based on social-indexical knowledge. Manuscript submitted for publication 


\section{Acknowledgments}

This thesis marks a particularly significant point in a long personal, academic, and intellectual journey. There have been so many people who have helped me along the way-many in ways I'm likely unaware of at this pointthat the task of properly acknowledging them is a little overwhelming.

I first want to thank the members of my committee: Florian Jaeger, Dick Aslin, Lori Holt, and Jeff Runner. Florian in particular has pushed me to take the ideas I've explored here much further than I thought they could go. I can't think of anyone with more enthusiasm for ideas, intellectual honesty, and broad curiosity.

The Rochester BCS community provided me a better intellectual home than I'd have imagined was possible. The kind of cross-disciplinary inquiry that first attracted me to cognitive science is embodied here in a truly special way. I particularly want to thank Robbie Jacobs for providing invaluable input on my projects at many points, despite the fact that I am a "language person" and he is a "vision person". I also want to thank Rajeev Raizada, who taught me everything I know about functional neuroimaging, and communicating my work in terms of clear, concrete metaphors.

Just as importantly, I would never have arrived at Rochester in the first place without the guidance of the many people who have mentored me over the years. During my fellowship at UMD, Bill Idsardi encouraged my curiosity 
and recognized, in a way that I didn't, that there's not as big a gulf between linguistics, Bayesian nonparametrics, and computational neuroscience as there might seem.

Further back, the Williams cognitive science community drew me into this field in the first place. Andrea Danyluk and Joe Cruz's captivating seminar introduced me to the combination of rigorous computational thinking applied to philosophically interesting questions that are grounded in human behavior that to this day exemplifies the intellectual potential of the field of cognitive science. Safa Zaki took a chance on an enthusiastic frosh who knew absolutely nothing about cognitive psychology as a summer research intern. Safa taught me that it's okay to mix behavioral and computational approaches. Moreover, without Safa's firm encouragement I doubt I would have ended up in grad school at all, and I consider myself deeply fortunate to count her as a friend and mentor.

Before that, a great many others have guided, supported, and mentored me. In the interest of space, I'll only thank Susan Thibodeau for telling me to shut up and listen more. A tough pill to swallow at the time, but it's advice that has served me extremely well.

None of this work would have been possible without the staff who keeps things running. Thanks to Kathy Corser, Jen Gillis, Chris Dambra, and Chris Freemesser in the BCS department, and to Andrew Watts in the HLP Lab. Thanks also to Judy Ripton, Pat Weber, and Evi Vanoost at the RCBI, and Donias Doko in the Raizada Lab.

Finally, my friends and family have had a role in this work in ways that are both tangible and intangible. Thanks, in no particular order, to Alex, Judith, Masha, Esteban, Dan, Ilker, Amanda, Lauren, Ruth, Liz, Greg, Lizz, Patty, Davis, Kevin, Tal, Keturah, Sarah, Irene, Cory, Klinton, Bozena, Darcy, Katie, Travis, Elika, Zach, Jue, Liz, Mom, Bob, Steph, Jeff, Eric, and Alyssa. I'm sure 
I've forgotten someone, please forgive me and chalk it up to sleep deprivation. Thanks, finally, to everyone at Joe Bean, Pour (Glen Edith), and Ugly Duck for keeping me sufficiently caffeinated, and to Morgan for running regular therapy sessions for years. 


\section{Abstract}

Perceptual systems have to make sense out of a world that is not only noisy and ambiguous, but that also varies from situation to situation. Human speech perception is a perceptual domain where this problem has long been acknowledged: individual talkers vary substantially in how they produce linguistic units using acoustic cues. Yet, how the speech system solves this problem of talker variability remains poorly understood. This thesis presents a computational framework - the ideal adapter-for understanding this problem and how the speech perception system solves it. The basic insight of this framework is that variability in speech is not arbitrary but rather structured: talkers are reasonably consistent in the way they produce cues, and individual talkers tend to cluster into groups by gender, regional background, etc. This structure means that listeners can use their previous experience with other talkers to guide perception of unfamiliar talkers, as well as familiar talkers that they encounter again. This framework unifies a large and messy literature on how listeners cope with talker variability, leads to quantitative models that provide good fits to human behavior in a variety of situations, and makes specific, testable predictions that open up new frontiers in understanding speech perception. This framework also applies to perception in general, and highlights how speech perception can serve as a model organism for understanding how perceptual systems cope with a variable but structured world. 


\section{Contributors and Funding Sources}

This thesis was supervised by a committee of Professors T. Florian Jaeger (advisor) and Richard Aslin of the Department of Brain and Cognitive Sciences, and Professor Lori Holt of the Department of Psychology at Carnegie Melon University. I am the primary author of the text of this dissertation. The work reported herein was completed in close collaboration with Florian Jaeger. Chapter 2 was published as Kleinschmidt and Jaeger (2015), and Chapter 3 was published as Kleinschmidt and Jaeger (2016a). Experiment 1 and the modeling work reported in Chapter 4 overlaps substantially with Kleinschmidt and Jaeger (2016b). The work reported in Chapter 5 was completed in collaboration with Florian Jaeger and Kodi Weatherholtz.

This research was supported by a Sproull Fellowship from the University of Rochester, an NSF GRFP (starting in 2011), and an NRSA F-31 grant from the National Institute of Child Health and Development (NICHD; starting in 2015) to the author, as well as NICHD R01 HD075797 and an Alfred P. Sloan Fellowship to T. Florian Jaeger. 


\section{Table of Contents}

Abstract

ix

Contributors and Funding Sources

$\mathbf{x}$

List of Tables

xiv

List of Figures

XV

1 Introduction $\quad 1$

1.1 Speech perception as a model organism . . . . . . . . . . 4

1.2 Summary of chapters . . . . . . . . . . . . 5

2 The ideal adapter: A framework for robust speech perception $\quad 8$

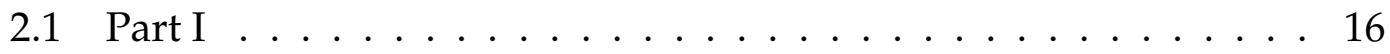

2.2 Part II .............................. 75

2.3 Part III . . . . . . . . . . . . . . . . . . 118 
3 Re-examining selective adaptation: Fatiguing feature detectors, or distributional learning?

3.1 Sensory adaptation as distributional learning . . . . . . . . . 147

3.2 Phonetic selective adaptation as distributional learning . . . . . 152

3.3 Challenges to this view $\ldots \ldots \ldots \ldots \ldots$

3.4 Discussion . . . . . . . . . . . . . . . 162

4 What do you expect from an unfamiliar talker? Inferring listeners' $\begin{array}{ll}\text { priors beliefs } & 171\end{array}$

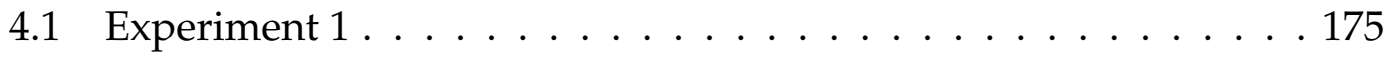

4.2 Inferring prior beliefs about talker variability $\ldots \ldots \ldots \ldots$. . . 182

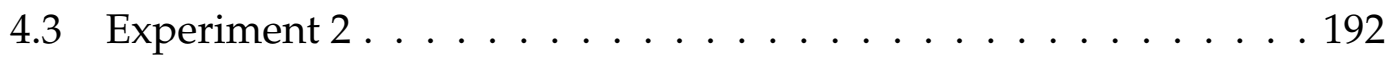

4.4 General discussion . . . . . . . . . . . . . . . . . 202

4.5 Conclusion . . . . . . . . . . . . . . . . . . . 209

5 Quantifying the role of socio-indexical structure in talker variation 211

5.1 General methods . . . . . . . . . . . . . . . . . . . . . 221

5.2 Study 1: Informativity of socio-indexical groupings about cue

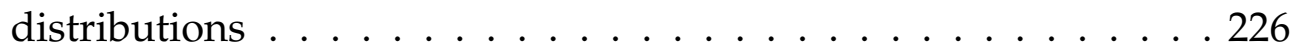

5.3 Study 2: utility of socio-indexical groupings for speech recognition 233

5.4 Study 3: Inferring socio-indexical variables from cue distributions 242

5.5 General Discussion . . . . . . . . . . . . . . . . . . . 245

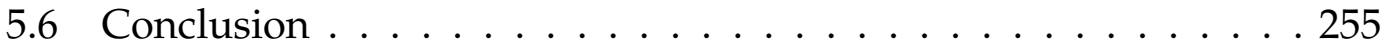

6 Conclusion 257

$\begin{array}{ll}\text { References } & 260\end{array}$ 
A Ideal adapter modeling methods and assumptions

A.1 Model specification . . . . . . . . . . . . . . . . . . 298

A.2 Audio-visual cue integration . . . . . . . . . . . . . 302

A.3 Model fitting and parameter estimation . . . . . . . . . . . 308

A.4 Model assumptions . . . . . . . . . . . . . . . . . . . . 314 


\section{List of Tables}

4.1 Percentage of boundary shift from typical talker to each exposure talker (see Figure 4.3), averaged over subjects with 95\% bootstrapped confidence intervals. . . . . . . . . . . . . . 181

4.2 Expected values and 95\% highest posterior density intervals for the prior parameters, given the adaptation data. . . . . . . . . . 190

A.1 Assumptions of the belief updating model used to evaluate the ideal adapter framework predictions. . . . . . . . . . . 317 


\section{List of Figures}

2.1 Distribution of frication frequency centroids, a crucial cue to the contrast between $/ \mathrm{s} /$ and $/ \mathrm{J} /$, from two talkers (Newman, Clouse, \& Burnham, 2001) . . . . . . . . . . . . . . . . . . . 10

2.2 Spectrograms for /aba/ (top) and /ada/ (bottom) with formant tracks (synthesized as described in Vroomen, van Linden, Keetels, de Gelder, and Bertelson (2004) and provided by Jean Vroomen). . . . . . . . . . . . . . . . . . . . . 21

2.3 Relationship between F2 locus likelihood functions $p(x \mid c)$ (top) and posterior probability of $/ \mathrm{b} /$, or classification function $p(c \mid x)$ (bottom), for three different talkers: a 'normal' talker (Norman), a 'shifted' talker (Sherman), and a 'precise' talker (Priscilla). . . . 27

2.4 Schematic illustration of the results of perceptual recalibration on classification of a /b/-/d/ continuum (A), and the changes in the listener's beliefs about the underlying distributions which we propose to account for the changes in classification (B). . . . 36

2.5 Recalibration results from Vroomen, van Linden, de Gelder, and Bertelson (2007), showing both the proportion of /b/ responses (top, solid line /b/ exposure and dashed line /d/ exposure) and the aftereffect difference score (bottom) for the first 64 critical exposures in the first exposure block. . . . . . . . . . . . . . . 39 
2.6 Belief updating model fit to build-up of recalibration data from Vroomen, van Linden, de Gelder, and Bertelson (2007). . . . . . . 43

2.7 The belief-updating model finds two different ways of fitting the build-up of recalibration (Figure 2.6), as illustrated by this density plot of the distribution of the mean and variance prior confidence parameters ( $\kappa_{0}$ and $v_{0}$, respectively) that are consistent with the data (estimated by samples via MCMC). . . . . . . . 46

2.8 Selective adaptation results from Vroomen, van Linden, de Gelder, and Bertelson (2007), showing both the proportion of /b/ responses (top) and the aftereffect difference score (bottom) for the first 64 cumulative exposures in the first exposure block. . 52

2.9 Schematic illustration of the results of selective adaptation on classification of a /b/-/d/ continuum (A), and the changes in the listener's beliefs about the underlying distributions which we propose to account for the changes in classification (B). . . . . 53

2.10 Belief-updating model fits to the data from Vroomen, van Linden, de Gelder, and Bertelson (2007) on the build up of selective adaptation after varying levels of cumulative exposure to a prototypical audio-visual adaptor ( $x$-axis, on a $\log$ scale) $\ldots \ldots$. . . 55

2.11 Schematic illustration of the predicted trade-off between shifts in mean and change in variance. . . . . . . . . . . . . . . 61

2.12 Results from Vroomen, van Linden, de Gelder, and Bertelson (2007), showing the full 256 exposures. . . . . . . . . . . . . 62

2.13 Results from fitting the belief updating model to all 256 exposures in both conditions from Vroomen, van Linden, de Gelder, and Bertelson (2007) simultaneously. . . . . . . . . . . . . 63 
2.14 Construction of stimuli for four conditions with visual /b/ (visual $/ d /$ is analogously the mirror image) . . . . . . . . . . 65

2.15 Results from all four conditions in the first exposure block: ambiguous, intermediate-ambiguous, intermediate-prototypical, and prototypical (colored lines with error bars showing 95\% confidence intervals). . . . . . . . . . . . . . . . . . . 68

2.16 The belief-updating model predicts when behavior should switch from recalibration-like to selective adaptation-like (see Figure 2.15). . . . . . . . . . . . . . . . . . . . 70

2.17 Talker-specific speech perception can be formalized as inference under uncertainty conditional on talker identity. . . . . . . . . 83

2.18 Adapting to multiple talkers: Updating of talker-specific beliefs allows listeners to continue to learn about individual talkers' generative models by accumulating evidence over multiple encounters. . . . . . . . . . . . . . . . . . . . 90

2.19 Two examples of how prior experience with many talkers of a language can constrain the space of generative models that has to be searched during adaptation. . . . . . . . . . . . . . 93

2.20 Prior beliefs at varying levels of specificity, formalized as conditional distributions over generative model parameters (plotted as ellipses of most probability mass). . . . . . . . . . . . . 98

3.1 Adaptor and test stimuli used by He, Kersten, and Fang (2012). . 150

3.2 Distributional learning accounts for the negative after-effects observed in phonetic selective adaptation. . . . . . . . . . . . 154

3.3 Selective adaptation data from Cole and Cooper (1977). . . . . . 159 
4.1 Each subject heard one of these five synthetic accents, which differ only in the distribution of VOTs of the word-initial stops. . . . 175

4.2 Example trial display (beach/peach). . . . . . . . . . 177

4.3 Listeners' responses, smoothed with logistic functions (thin lines), compared with the classification functions expected based on a typical talker (no learning; dashed black lines) and complete (but not necessarily optimal) adaptation to the exposure distributions (thick dashed colored lines). . . . . . . . . 179

4.4 Most listeners' individual boundaries fall between the boundaries implied by cue distributions from a typical talker and exposure talker. . . . . . . . . . . . . . . . . . . 180

4.5 The classification functions (shaded ribbons, 95\% posterior predictive intervals) predicted by the belief updating model fit listeners' responses well (dots with lines showing bootstrapped $95 \%$ confidence intervals). . . . . . . . . . . . . . . 187

4.6 Expected cue distributions based on the prior beliefs inferred here from behavioral adaptation data. . . . . . . . . . . 188

4.7 In Experiment 2, each subject heard a talker that produced one of these five VOT distributions. . . . . . . . . . . . . . . . . . . 194

4.8 Category boundaries estimated during post-test are correlated with estimates from exposure (in the 10,50 condition where such an estimate is possible), but more variable. . . . . . . . . . 196

4.9 Listeners' categorization functions in Experiment 2 (during posttest) reflect partial adaptation to the exposure talker, especially for more extreme conditions extreme distributions. . . . . . . . . 197 
4.10 The distribution of listeners' individual category boundaries in Experiment 2 reflects partial adaptation to the exposure talker's VOT distributions. . . . . . . . . . . . . . . . . . . . . . 198

4.11 The prior beliefs inferred from Experiment 1 predict how much listeners adapt to each of the input distributions of Experiment 2. 200

5.1 Socio-indexical variables are more informative about cue distributions for vowel (formants) than for stop voicing (vot). . . . . 228

5.2 Male and female distributions of Normalized F1xF2 diverge from the marginal distributions (A) only slightly less than talker-specific VOT distributions diverge from marginal (B) (see Figure 5.1 . . . . . . . . . . . . . . . . . . 230

5.3 Individual vowels vary substantially in the informativity of grouping variables about their cue distributions. . . . . . . . . 231

5.4 A small number of dialect/vowel combinations account for most of the divergence of dialect-specific vowel formant distributions. 232

5.5 Speech recognition accuracy using for marginal, group-level, and talker-specific cue distributions. . . . . . . . . . . . . . . 239

5.6 Probability of correct recognition varies across vowels, overall and according to the socio-indexical grouping variable. . . . . . 241

5.7 The utility of socio-indexical variables varies across dialects. . . . 242

5.8 Probability of correctly classifying a talker's socio-indexical group varies with the grouping variable, contrast, and cues. . . . 244

A.1 MCMC-estimated joint posterior density (contours) and MAPestimates (asterisks) of prior confidence parameters for all studies presented in the main text. . . . . . . . . . . . . . . 315 


\section{Introduction}

Perception is one of the basic components of intelligent behavior. In order to behave intelligently, an agent (person, animal, robot) needs to adjust their behavior based on the state of the world. But agents do not have direct access to behaviorally relevant facts about the state about the world. Rather, they receive sensory signals, transmitted by sense organs, and the problem of perception is to extract meaningful information from these signals. The mapping from sensory signals to meaningful facts about the world is not straightforward. There are, for instance, infinitely many three dimensional shapes that generate the same retinal image. In this sense perception is a process of inference under uncertainty, of determining which possible state of the world best explains the received signals (von Helmholtz, 1924).

One fruitful tradition for understanding how perception works has been to characterize this inference problem itself, since the constraints imposed by this problem will shape the design and implementation of any system that solves it successfully (Marr, 1982; Knill \& Richards, 1996). A central motivation behind this tradition is that the mind is shaped by-and hence adapted to- the world it has to function in. In this view, perceptual inference depends in large part on knowledge about the statistical properties of sensory signals. The statistics of the sensory world determine, for instance, which aspects of sensory signals are 
informative and which are noise. Indeed, perceptual sensitivities-measured both neurally and behaviorally - can in many cases be directly derived from the relevant sensory statistics (Attneave, 1954; Atick \& Redlich, 1990; Olshausen \& Field, 1996; E. C. Smith \& Lewicki, 2006; Lewicki, 2002; Simoncelli \& Olshausen, 2001; Field, 1987).

The statistics of the sensory world also determine how any particular sensory signal should be interpreted. At the most basic level, the overall light level determines which signals correspond to "dark" and which to "light". This leads to a corollary to the link between sensory statistics and the perceptual system: in a world where sensory statistics change from one situation to the next, the perceptual system must adapt to these changes. Perceptual systems are, in fact, extremely adaptable, and this adaptation has been linked to changes in sensory statistics (Barlow \& Földiák, 1989; Brenner, Bialek, \& de Ruyter Van Steveninck, 2000; Fairhall, Lewen, Bialek, \& de Ruyter Van Steveninck, 2001; Gutfreund, 2012; Sharpee et al., 2006; Webster, Werner, \& Field, 2005; Wei \& Stocker, 2012; Kohn, 2007). This work suggests that, at the very least, perceptual systems are tracking changes in sensory statistics.

Like perception itself, adaptation can be thought of as a kind of inference, inference about the statistical properties of the sensory world (Fairhall et al., 2001; Seydell, Knill, \& Trommershäuser, 2010). Each sensory signal provides some information about the underlying statistics of the current environment, and together are more or less well-explained by different possible distributions of sensory signals, much like each signal itself is more or less well-explained by different states of the world. And, just like perceptual inference depends on statistical properties of the world, this higher-level inference depends on higherorder statistical properties of the world.

This raises the central question addressed by the work reported here: is tracking the best way to think about how perceptual systems cope with a dy- 
namic world? Existing theories tend to focus on either continuously tracking sensory statistics (Fairhall et al., 2001) or detecting changes and re-learning statistics in response (e.g., Courville, Daw, \& Touretzky, 2006; Gallistel, Mark, King, \& Latham, 2001; Dayan \& Niv, 2008). The particular way that the statistics of the sensory world vary determines how the perceptual system can best cope with these changes. Continuous tracking is the best you can do when statistics change continuously and unpredictably. When changes in statistics are caused by differences between contexts, but the contexts themselves vary in unpredictable ways, the best you can do is to detect context changes and re-learn the context-specific statistics when one occurs. Both of these strategies can be thought of as flat learning models, which do not learn any cross-context structure.

However, in a multi-context world, this kind of flat learning is sub-optimal. If there's a chance you will encounter a particular context again, you should not forget it as soon as you enter a new context (cf. Qian, Jaeger, \& Aslin, 2012). Rather, by learning how statistical contingencies vary across contexts, you can take advantage of prior experience with a context when you next encounter it. Agents do, in fact, often encounter the same context repeatedly, and there is abundant evidence that when the context changes, learners do not forget old statistics (Thanellou \& Green, 2011; Bouton \& King, 1983; Sissons \& R. R. Miller, 2009; Körding, Tenenbaum, \& Shadmehr, 2007; Knudsen, 1998; Linkenhoker, von der Ohe, \& Knudsen, 2005). A multi-context world thus requires inference at three levels. First, agents must make inferences about behaviorally relevant properties of the world. These inferences depend on knowledge of the generative model that links those properties with observable sensory signals. Second, in a non-stationary world, agents must also make inferences about the current context's generative model. Third, in a multi-context world where cross-context variation is structured, agents must also infer which context 
they are currently in, and how it relates to previous contexts.

\subsection{Speech perception as a model organism}

While the questions addressed in this work apply to perception generally, it focuses on speech perception as a test case or "model organism" for understanding perception in a variable world. Speech perception makes for a good model organism in large part because as a perceptual problem it exemplifies the three levels of inference required for perception in a variable but structured world.

First, each phonetic category is realized as a distribution of acoustic cues (e.g., Lisker \& Abramson, 1964; Peterson \& Barney, 1952; Allen, J. L. Miller, \& DeSteno, 2003; Newman, Clouse, \& Burnham, 2001). This means that speech perception can be understood and modeled as problem of inference under uncertainty (Clayards, Tanenhaus, Aslin, \& Jacobs, 2008; Norris \& McQueen, 2008). Second, the statistical properties of speech are non-stationary, and the distributions of acoustic cues change from situation to situation (Peterson \& Barney, 1952; Allen, J. L. Miller, \& DeSteno, 2003; Jongman, Wayland, \& Wong, 2000; McMurray \& Jongman, 2011; Newman et al., 2001; Hillenbrand, Getty, Clark, \& Wheeler, 1995). This has long been recognized as a central challenge to successful speech perception (cf. the "lack of invariance", Liberman, Cooper, Shankweiler, \& Studdert-Kennedy, 1967). Third, this cross-situational variability is highly structured. Individual talkers serve as contexts which correspond to relatively stable cue distributions (Heald \& Nusbaum, 2015). Moreover, talkers cluster into groups based on factors like gender, regional origin, class, etc. (Clopper, Pisoni, \& de Jong, 2005; Heald \& Nusbaum, 2015; Labov, 1972; Labov, Ash, \& Boberg, 2005; Eckert \& McConnell-Ginet, 2013, among others).

This structure is not just present in the world. Listeners are exquisitely sen- 
sitive to this structure, and use it to guide their inferences about the underlying linguistic categories (Johnson, Strand, \& D'Imperio, 1999; Strand \& Johnson, 1996; Hay, Nolan, \& Drager, 2006; Hay \& Drager, 2010; Creel \& Bregman, 2011; Creel \& Tumlin, 2011; Creel, Aslin, \& Tanenhaus, 2008; Niedzielski, 1999; Nygaard \& Pisoni, 1998; Sumner \& Samuel, 2009). On a practical level, we know a great deal about both the linguistic and indexical structure of the speech signal (e.g., to a first order of approximation, what the categories and relevant cues are, on the one hand, and how the realizations vary across talkers and within groups). This enables us to probe how speech perception copes with different kinds of variation at a behaviorally relevant, ecologically valid level.

What is currently lacking in the understanding of speech perception is a theoretical framework for linking these together. As I discuss in Chapters 2 and 3, existing theoretical approaches to speech perception miss important aspects of variability in speech perception, and cannot account for the full range of strategies that listeners employ to cope with this variability. Thus, the main goal of this dissertation is to develop a theoretical framework for speech perception that takes both variation and structure seriously. This framework motivates further empirical and modeling work. Later chapters take first steps in a number of these directions.

\subsection{Summary of chapters}

Chapter 2 introduces the ideal adapter, a novel computational framework for understanding speech perception in the face of non-stationarity. This framework offers a solution to the longstanding problem of talker variability in speech perception, providing an explanation for the range of strategies that listeners use to cope with variability in the statistics of speech sounds. These strategies range from rapidly adapting to unfamiliar talkers to recognizing familiar talkers and 
generalizing to talkers who are similar to previously encountered talkers. The central idea of this framework is that because of the nature of the speech signal, robust speech perception requires that listeners simultaneously infer what is being said, how linguistic units are realized probabilistically in the current context, and who the current talker is, in relation to other talkers. Formalizing this as hierarchical probabilistic inference, this chapter shows that a simple belief updating model captures a range of rapid adaptation phenomena, and effectively predicts adaptation in novel, previously untested conditions. The rest of this chapter lays out, at a conceptual level, how treating speech perception as a hierarchical inference process unifies the large and often superficially contradictory literature on how listeners cope with talker variability. At the same time, it develops novel predictions that open up directions for future work. The chapter closes by considering the broader relevance of this framework speech perception and language comprehension more broadly. This chapter was published as Kleinschmidt and Jaeger (2015).

Chapter 3 explores the connection between distributional learning, sensory adaptation, and speech perception. One of the surprising findings from Chapter 2 is that selective adaptation, a phonetic adaptation phenomenon typically attributed to fatigue of acoustic-phonetic feature detectors, might better be explained as a process of distributional learning, where listeners update their beliefs about the underlying distributions of acoustic cues. General sensory adaptation, like phonetic selective adaptation, was also historically treated as the fatigue of feature detectors, but recent work, paralleling that of Chapter 2, suggests that sensory adaptation, too, might better be thought of as a process of distributional learning. This chapter develops this parallel further, reviews the developments in our understanding of sensory adaptation, and critically re-examines evidence that phonetic selective adaptation is not distributional learning. This chapter was published as Kleinschmidt and Jaeger (2016a). 
Chapter 4 investigates the role that listeners' prior expectations play when adapting to an unfamiliar talker. The ideal adapter predicts that adaptation should be constrained by listeners' prior experience with how much and in what ways talkers vary in the cue distributions that they produce. This chapter presents two experiments. The first experiment shows that, as predicted, listeners do not adapt as well to a talker whose "accent" is outside the range of normal variation across talkers. Next, this chapter shows how we can use the computational framework of the ideal adapter to work backwards from these constraints to infer what listeners' prior expectations are for an unfamiliar talker. The second experiment validates the findings of the first, using more extreme distributions, and also validates the predictive accuracy of the prior beliefs inferred on the basis of the first experiment.

Chapter 5 goes the opposite direction from Chapter 4, examining the structure in how talkers actually vary in order to enable better predictions about what groupings listeners might pick up on. The ideal adapter predicts that listeners pick up on structure of how talkers vary in the world, and use this to guide adaptation. The particular structure that listeners are predicted to extract depends on how informative different groupings of talkers are about their underlying cue distributions. This chapter uses the computational tools of ideal listener/adapter approaches to quantify the informativity of socio-indexical grouping variables for different phonetic categories and cues. Having such data enables more specific predictions about what kinds of grouping listeners should track, and hence how they can best generalize from their experience with one talker to another.

Finally, chapter 6 provides brief concluding remarks. 


\section{The ideal adapter: A framework for robust speech perception ${ }^{1}$}

In order to understand speech, listeners have to map a continuous, transient signal onto discrete meanings. This process is widely assumed to involve the recognition of discrete linguistic units, such as phonetic categories, words, and sentences. The relative stability with which we usually seem to recognize these units belies the formidable computational challenge that is posed by even the recognition of the smallest meaning distinguishing sound units (such as a /b/ or $/ \mathrm{p} /$ ). In this paper, we characterize this computational problem and propose how our speech processing system overcomes one of its most challenging aspects, the variability of the signal across different situations (e.g., talkers). This problem is not unique to speech recognition, but is a general property of inferring underlying categories and intentions in a changing (i.e., subjectively non-stationary) world (see references in Qian et al., 2012). The framework that we propose here thus has broad relevance for understanding how people manage changes in the statistical properties of stimuli across different perceptual and cognitive tasks.

The recognition of phonetic categories is broadly assumed to involve the

\footnotetext{
${ }^{1}$ The contents of this chapter were previously published as Kleinschmidt, D. F. \& Jaeger, T. F. (2015). Robust speech perception: Recognize the familiar, generalize to the similar, and adapt to the novel. Psychological Review, 122(2), 148-203. doi:10.1037/a0038695
} 
extraction and combination of acoustic and, if present, visual cues. This is a complex task for several reasons. The speech signal is both transient and typically unfolds at speeds not under the listener's control. Additionally, perceptual cues to phonetic categories are often asynchronously distributed across the speech signal. That means that some cues to a phonetic category contrast are detectable several syllables in advance of the phonetic segment, while at the same time cues following a segment can still be informative (e.g., rhoticity, Heid \& Hawkins, 2000; Tunley, 1999). Beyond the extraction of acoustic cues from the speech signal, there are two problems which have puzzled researchers for decades. First, the mapping from cues to phonetic features or phonetic categories is non-deterministic: from the perspective of a listener, phonetic categories form distributions over multiple cue dimensions, and these distribution overlap with those of other categories. Notably, even multiple instances of the same phonetic category produced by the same talker in the same phonetic context will have different physical properties (Allen, J. L. Miller, \& DeSteno, 2003; Newman et al., 2001). One cause for these distributions is noise in the biological systems underlying linguistic production (e.g., motor noise in the articulators). Similarly, the perceptual system itself is noisy: neurons that respond to certain acoustic features do not deterministically fire when that feature is present (Ma, Beck, Latham, \& Pouget, 2006). Additionally, the acoustic properties of the environment like background noise can further alter the linguistic signal.

However, arguably the biggest challenge to speech perception is that the mapping from acoustic cues to phonetic categories can vary across situations. A 'situation' could be characterized in terms of an individual talker or a group of talkers with a similar way of speaking, or other aspects of the environment which lead to systematic changes in speaking style (like a noisy bar). For example, different talkers sometimes realize even the same phonetic categories, in the same phonetic context, with dramatically differently cue distributions 

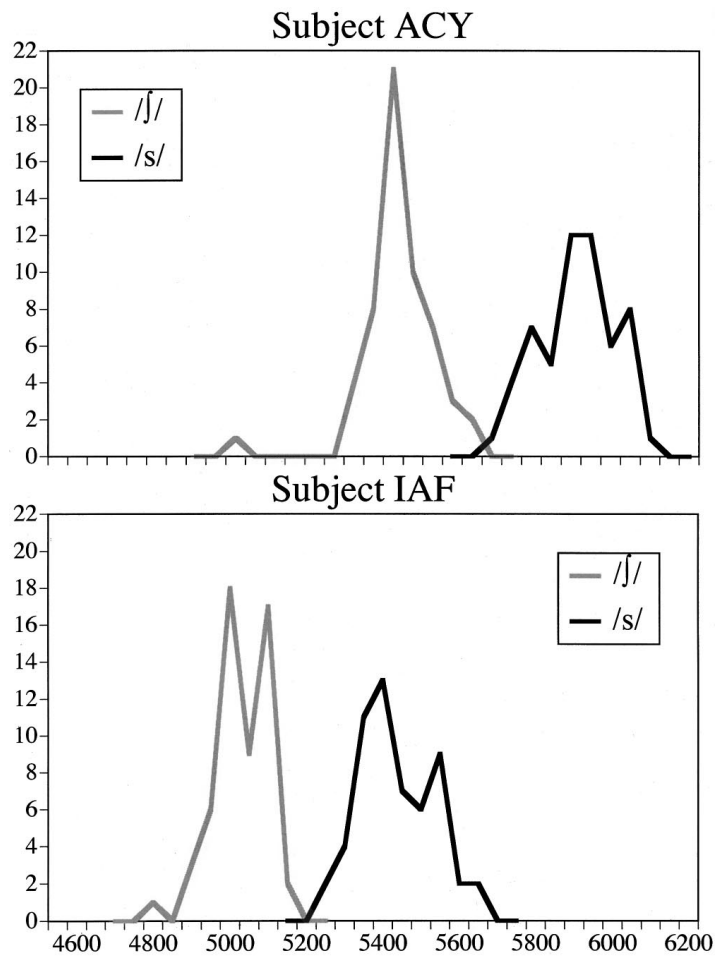

Figure 2.1: Distribution of frication frequency centroids, a crucial cue to the contrast between $/ \mathrm{s} /$ and $/ \mathrm{J} /$, from two talkers (reproduced with permission from Newman et al., 2001, copyright 2001 Acoustical Society of America).

(e.g., Allen, J. L. Miller, \& DeSteno, 2003; McMurray \& Jongman, 2011; Newman et al., 2001). These differences might arise from fixed, physical differences in, for instance, vocal tract size, but they also arise from variable or stylistic factors like language, dialect, or sociolect (e.g., Babel \& Benjamin Munson, 2014; Johnson, 2006; Labov, 1972; Pierrehumbert, 2003). These differences in the cueto-category mapping can be substantial. Figure 2.1 shows the distributions of one of the primary cues distinguishing between $/ \mathrm{s} /$ and $/ \mathrm{J} /$ as produced by two different talkers. Such between-talker variability means that one talker's "ship" is physically more like another's "sip". This problem is known as the lack of invariance and is one of the oldest problems in speech perception (Liberman et al., 1967). The focus of this article is how listeners manage to accommodate such systematic variability and achieve robust speech recognition. 


\section{Overcoming the lack of invariance: The proposal}

In the face of the sort of variability between situations-talkers, in this caseseen in Figure 2.1, it is natural to wonder how we can understand each other at all. We propose that the answer to this question is three-fold:

1. recognize the familiar,

2. generalize to the similar, and

3. adapt to the novel.

As we discuss below, at least the first and the last of these have been more or less explicitly assumed in previous work, and there is at least preliminary evidence for the second. In a familiar situation, the speech recognition system has a great deal of previous experience to draw on, and by recognizing a familiar situation it can take advantage of this previous experience. Recognition of the familiar underlies, for example, talker-specific interpretation of the acoustic signal (Creel, Aslin, \& Tanenhaus, 2008; Eisner \& McQueen, 2005; Goldinger, 1998; Kraljic \& Samuel, 2007; Nygaard \& Pisoni, 1998). Similarly, generalizing to a novel situation based on similar previous experience means the speech recognition system doesn't have to start from scratch each time a new situation is encountered. For example, such generalization allows us to recognize an accent and adjust our interpretations based on previous experience with similar talkers (Baese-berk, Bradlow, \& Wright, 2013; Bradlow \& Bent, 2008; Sidaras, Alexander, \& Nygaard, 2009). At the same time, novel situations might require adaptation beyond what is expected based on previous experience. For example, when encountering a talker with a novel dialect or accent, the speech recognition system must be prepared to adapt rapidly and flexibly.

We propose that all three of these strategies arise from the function that the speech recognition system fulfills (i.e., the typical goals of speech recognition), 
and that the basic design of this system reflects the fact that it must function efficiently under normal circumstances. Specifically, we propose that the three strategies emerge from the organizational constraints on the speech recognition system imposed by the presence of variability both within a single situation and between situations. These constraints lead naturally to a few conceptual components for the proposed framework. First, because there is variability within a situation, the mapping between cues and categories is inevitably probabilistic. This makes speech recognition a problem of inference under uncertainty and implies that a robust speech recognition system must use distributional (statistical) knowledge (Clayards et al., 2008; Feldman, Griffiths, \& Morgan, 2009; Norris \& McQueen, 2008).

Second, because cue distributions themselves vary-sometimes unpredictably-across situations, the system must be prepared, when necessary, to engage in distributional/statistical learning. This is closely related to the notion of life-long implicit learning (Botvinick \& Plaut, 2004; Elman, 1990; Chang, Dell, \& Bock, 2006), as well as statistical learning theories of language acquisition (Feldman, Griffiths, Goldwater, \& Morgan, 2013; McMurray, Aslin, \& Toscano, 2009; Vallabha, McClelland, Pons, Werker, \& Amano, 2007), a connection we return to below.

Third, cue distributions do not vary arbitrarily across situations. Rather, the world is structured. For instance, a listener is likely to encounter a particular familiar talker's cue distributions again, relative to any arbitrary cue distributions, and likewise they are more likely to encounter cue distributions that are similar to those encountered in the past, because of regularities in how talkers vary within a language or more specific grouping like gender, dialect, accent, etc. We propose that in order to take advantage of such structured variability, the speech perception system does not only engage in distributional learning. In its most basic sense, this is demonstrated by our ability to recognize previ- 
ously encountered talkers, and use talker-specific experience to guide speech perception. Going beyond talker-specificity, we will discuss evidence that argues for sensitivity to structure over groups of talkers or situations. In a world where speech statistics vary in structured ways, life-long adaptation alone is not sufficient for robust speech perception. A robust speech perception system should take advantage of structure in the world that allows previous experience to inform current processing (for similar reasoning applied to other cognitive domains, cf. Qian et al., 2012). It is, we propose, sensitivity to this structure in the world that underlies recognition of familiar situations and generalization to similar ones.

In this paper, we elaborate on this proposal, review the relevant literature, and develop a framework in the tradition of ideal observer models and normative/Bayesian inference (Anderson, 1990) that, we hope, will help guide future work on speech perception. As we detail below, the proposed framework, which we dub the ideal adapter, understands all of (1) to (3) above (i.e., recognition, generalization, and distributional learning) as the result of selecting and adapting the appropriate generative model for the current situation based on the integration of prior and present experience (hence, the name for the proposed framework). This brings a unifying and-at least in parts-formalized computational framework to a set of ideas that have been assumed-more or less explicitly - by others before us. For example, it is widely assumed that speech perception is talker-specific (e.g., Creel \& Bregman, 2011; Pardo \& Remez, 2006; Pisoni \& Levi, 2007) and recent work has begun to investigate our ability to generalize across talkers (e.g., Bradlow \& Bent, 2008; Eisner \& McQueen, 2005; Kraljic \& Samuel, 2007; Sidaras, Alexander, \& Nygaard, 2009). The ideal adapter framework ties together these different lines of work, emphasizing the crucial roles of both the structure of listeners' prior knowledge and their ability to learn the statistics of novel situations. It has so far not been 
fully recognized, we submit, just how far-reaching the consequences of these two aspects of speech recognition are. Laying out the consequences of these two aspects of the framework thus forms the core of this article.

We begin our exposition in Part I with adaptation to the novel. For this, we focus on situations in which listeners have high certainty (i.e., 'know') that they need to adapt. We formalize the problem of adaptation and test the predictions of the ideal adapter framework through an implemented model. We focus on two well-studied phonetic adaptation phenomena: the first where listeners recalibrate one phonetic category in response to auditorily ambiguous stimuli labeled as one category (perceptual recalibration or phonetic adaptation, Bertelson, Vroomen, \& de Gelder, 2003; Kraljic \& Samuel, 2005; Norris, McQueen, \& Cutler, 2003), and the second where listeners change their classification behavior after repeated exposure to the same prototypical stimulus (selective adaptation, Eimas \& Corbit, 1973; Samuel, 1986). This leads us to develop and test novel predictions of the proposed framework. In this part of the paper, we spend a substantial amount of time developing intuitions about the mechanics of how-in the ideal adapter framework-listeners can update their beliefs about the cue distributions in the current situation based on direct experience. In doing so, we illustrate how the proposed perspective relates to and diverges from standard accounts of speech recognition.

In Part II, we turn to situations where previous knowledge is crucial for robust speech perception: recognition of familiar situations and generalization to similar novel situations. In contrast to the flexibility demanded by novel situations, in familiar situations listeners can benefit from stable representations of past experience. The ideal adapter framework provides a natural link between the distribution of speech statistics in the world-at the level of individual talkers and groups (e.g., dialect, gender, language)—and different strategies for how listeners can achieve robust speech perception in the face of the lack 
of invariance. In Part II we will discuss what structure there is in the world that listeners can take advantage of and review the evidence that they do take advantage of it. In doing so, we identify directions for future research and isolate a number of specific questions that we consider particularly critical for our understanding of the human speech recognition system.

Finally, we close in Part III by putting the framework we have developed into broader perspective. In particular, we will address how our approach relates to other approaches to the problem of the lack of invariance. Following that, we will discuss how our framework might inform broader issues in speech perception, language comprehension, and more domain-general learning and adaptation. Our approach is a computational-level one and as such compares only indirectly to mechanistic- or algorithmic-level approaches (Marr, 1982), but it nevertheless provides a set of tools for reasoning about speech perception (and language comprehension more generally) which can help sharpen questions for research at other levels. For example, the questions raised by the ideal adapter framework also speak to the debate between episodic, exemplarbased or more abstract phonetic representations (Johnson, 1997b; Goldinger, 1998; McClelland \& Elman, 1986; Norris \& McQueen, 2008; Pierrehumbert, 2003). They also relate to the acquisition of phonetic categories, which can be seen as another type of distributional learning problem (Maye, Werker, \& Gerken, 2002; McMurray, Aslin, \& Toscano, 2009; Vallabha et al., 2007), and to language processing at higher levels (e.g., Fine, Jaeger, Farmer, \& Qian, 2013; Grodner \& Sedivy, 2011; Kamide, 2012; Kurumada, Brown, \& Tanenhaus, 2012; Kurumada, Brown, Bibyk, Pontillo, \& Tanenhaus, 2014). We also discuss recent research that has found adaptive behavior in language processing above the level of speech perception. At its most basic, the ideal adapter framework also contributes to the burgeoning literature on learning in a variable world (e.g., change detection Gallistel et al., 2001; hierarchical reinforcement learn- 
ing Botvinick, 2012; motor learning Körding, Tenenbaum, \& Shadmehr, 2007). Along with other recent approaches, the ideal adapter stresses that the crosssituational statistics of the world-though being variable-are structured, and that our cognitive systems have evolved to take advantage of this structure.

\subsection{Part I}

\subsubsection{The ideal adapter framework}

Adaptation in speech perception has received a great deal of attention recently. For example, when listeners initially encounter accented speech, they process it more slowly and less accurately, but this disadvantage dissipates within a matter of minutes (Bradlow \& Bent, 2008; Clarke-Davidson \& Garrett, 2004, and references therein). Similarly, listeners rapidly adapt to synthesized and otherwise distorted speech (e.g., Davis, Johnsrude, Hervais-Adelman, Taylor, \& McGettigan, 2005). Adaptation is not limited to cases of highly unusual pronunciation, such as foreign accents. Even relatively subtle divergences from standard cue distributions can lead to adaptation. For example, listeners adapt to a talker who produces cue distributions with a typical mean value but less variability than normal (Clayards et al., 2008). This suggests that continuous and implicit adaptation to subtle deviations from auditory expectations is a pivotal component of the human speech perception systems.

What is lacking thus far, however, is a better understanding of how and when we adapt. Specifically, how do listeners detect that their current linguistic representations are inadequate for the current situation, and how is evidence from the currently processed speech stream integrated with previous experience in order to achieve adaptation? Despite the central importance of the lack of invariance problem to speech perception and language understanding (Liberman 
et al., 1967; Pardo \& Remez, 2006), to date there are few cognitive models of adaptation, and as we discuss below, those that do exist do not link it to other strategies for dealing with the lack of invariance. State of the art models of speech perception have begun to address the non-determinism inherent in the mapping from cues to categories, but ignore or abstract away from the specific contributions of the lack of invariance (Feldman, Griffiths, \& Morgan, 2009; Feldman, Griffiths, Goldwater, \& Morgan, 2013; Norris \& McQueen, 2008). ${ }^{2}$

We propose that the first important step is to ask why phonetic adaptation occurs at all, or rather why one would expect speech perception to exhibit adaptive properties. To that end it is helpful to understand speech perception as a problem of inference under uncertainty. The acoustic cues that provide information about the talker's intended message are variable and ambiguous, and thus each individual cue is only partially informative. In order to effectively infer the underlying message, information must be integrated from many sources, and as we will discuss below, this must be guided by knowledge of the distribution of cues associated with each linguistic unit. However, because of the lack of invariance, these distributions differ across situations (e.g., talkers).

\section{Adaptation through belief updating}

Our central proposal is twofold. First, listeners do not have direct access to the true distribution but rather uncertain beliefs about them based on a limited number of observations. Second, inaccurate beliefs about the underlying distributions can lead to slowed or inaccurate phonetic categorization, and in order to achieve robust speech perception across situations, listeners must adapt. In this view, adaptation reflects a sort of incremental distributional learning, and

\footnotetext{
${ }^{2}$ In work on automatic speech recognition, however, the interest in talker-independent speech recognition has led to a range of proposals that are similar in varying degrees to what we propose here for human speech recognition (e.g., Gauvain \& Chin-Hui Lee, 1994; Leggetter \& Woodland, 1995; Shinoda \& C.-H. Lee, 2001).
} 
such distributional learning can be computationally characterized as belief updating. This incremental distributional learning has to integrate recent experience with a novel situation with prior knowledge and assumptions about the language. In this sense, the proposed account builds on and expands on the general idea of life-long implicit learning (Botvinick \& Plaut, 2004; Chang et al., 2006; Elman, 1990) and that the processing of language input is inevitably tied to implicit learning (e.g., Clark, 2013; Dell \& Chang, 2014; Jaeger \& Snider, 2013). Unlike much of this work, however, we will argue that the implicit beliefs listeners hold based on previous experience are not unstructured. Rather, they reflect higher-level knowledge (beliefs) about different talkers, groups of talkers, dialect and accents, and so on. We return to this in the second part of the paper.

The first proposition of our framework is that human speech perception relies on a generative model, or the listener's knowledge of how linguistic units (words, syllables, biphones, phonetic categories, etc.) are realized by different distributions of acoustic cues. Such knowledge allows for speech perception to proceed by comparing how well each possible explanation-higher-level linguistic unit-predicts the currently observed signal. The proposal that language comprehension proceeds via prediction of the signal accounts for a variety of properties of language understanding beyond the ones discussed here (cf. Dell \& Chang, 2014; Farmer, Brown, \& Tanenhaus, 2013; Jaeger \& Snider, 2013; M. C. MacDonald, 2013; Pickering \& Garrod, 2013) and is closely related to similar proposals from visual perception and other domains (Clark, 2013; Friston, 2005; Hinton, 2007; Y. Huang \& Rao, 2011; Rao \& Ballard, 1999).

Our second proposition is that the cue values predicted from a given linguistic unit depend not only on what is being said (the phonetic category, biphone, word, etc.) but also on who is saying it, and good speech perception depends on using an appropriate generative model for the current talker, regis- 
ter, dialect, etc.. The listener never has access to the true generative model, but rather only their uncertain beliefs about that generative model. Thus, adaptation can be thought of as an update in the listener's talker- or situation-specific beliefs about the linguistic generative model. The idea that speech adaptation reflects learning about the linguistic generative model is not in and of itself novel, but it has largely been implicit in the empirical literature thus far and our proposal provides an explicit framework and formalization for understanding the link between learning and processing in speech perception.

Our goal is to provide a framework for understanding, on the one hand, how listeners might best represent past experience with different situations, and on the other hand how listeners can integrate that previous experience with evidence from the currently processed speech signal in order to infer an appropriate generative model for each situation. As we discuss below, the listener needs to bring their beliefs about the distribution of cue values for each category into alignment with the actual distribution that the talker is producing. Because the speech signal unfolds over time, and because the fine-grained acoustic information fades rapidly, this belief updating must be done incrementally. However, this is difficult because each individual speech sound is corrupted by the intrinsic variability of the speech production, transmission, and perception process, and hence not an unambiguous cue to the underlying distribution. That is, when a listener hears a cue value that they do not expect, it could be due either to a change in the underlying distributions, or because deviations from prototypical cue values happen for a variety of other reasons (muscle fatigue, coarticulation, background noise, etc.). The question is thus how the listener should incorporate each new piece of evidence into their beliefs. We address this question by developing an ideal adapter framework, which, in the tradition of computational-level/rational analysis (Anderson, 1990; Marr, 1982), sets out the statistically optimal way to do this integration. By this, we 
mean that adaptation reflects an inference process which combines prior beliefs and recent experience proportional to the degree of confidence in each. ${ }^{3}$

\section{The ideal listener or phonetic categorizer}

Our ideal adapter framework builds on a foundation of ideal listener models, which describe the problem of speech perception as statistical inference of the talker's intentions (Clayards et al., 2008; Feldman, Griffiths, \& Morgan, 2009; Norris \& McQueen, 2008; Sonderegger \& Yu, 2010). Such inference includes inferring intermediate linguistic units, like phonetic categories, either as a means to another end or as an end in itself, as in the case of explicit experimental phonetic categorization tasks. Because we focus specifically on the problem of inferring phonetic categories, for our purposes an ideal listener model is better characterized as an ideal phonetic categorizer model. For the broader goal of understanding speech perception, it should, however, be kept in mind that the human listener is not just a phonetic categorization machine, and phonetic categorization typically serves other ends (such as lexical access, Norris \& McQueen, 2008; or even the successful inference of communicative intentions Jaeger \& Ferreira, 2013).

Because of the inherent variability of how a phonetic category is realized acoustically, any particular cue value is in principle ambiguous, and thus phonetic categorization is a problem of inference under uncertainty. Such inference can be formally expressed in the language of Bayesian statistics. In the general case, the posterior probability of each category $C=c_{i}$ after observing cue value

\footnotetext{
${ }^{3}$ This is not intended as a claim that such inference is resource-free or that there is unlimited memory. Importantly, there are many cognitively plausible algorithms which provably approximate-in principled ways-the type of rational inference assumed here for simplicity's sake, and do so with limited resources (e.g., Sanborn, Griffiths, \& Navarro, 2010). Many of these algorithms are closely related to mechanistic models like exemplar models (see Gibson, Rogers, \& Zhu, 2013; Shi, Griffiths, Feldman, \& Sanborn, 2010). We return to this point in the final part of this article.
} 
$x$ is related to the prior probability of $c_{i}, p\left(C=c_{i}\right)$ and the likelihood of $x$ under category $c_{i}, p\left(x \mid C=c_{i}\right)$, according to Bayes rule:

$$
p\left(C=c_{i} \mid x\right)=\frac{p\left(x \mid C=c_{i}\right) p\left(C=c_{i}\right)}{\sum_{j} p\left(x \mid C=c_{j}\right) p\left(C=c_{j}\right)}
$$

Because the denominator of the fraction does not depend on the specific category $c_{i}$ and only serves to ensure that all of the posterior probabilities $p(C=$ $\left.c_{i} \mid x\right)$ sum up to one, it is often omitted and the relationship is written as proportionality:

$$
p\left(C=c_{i} \mid x\right) \propto p\left(x \mid C=c_{i}\right) p\left(C=c_{i}\right)
$$
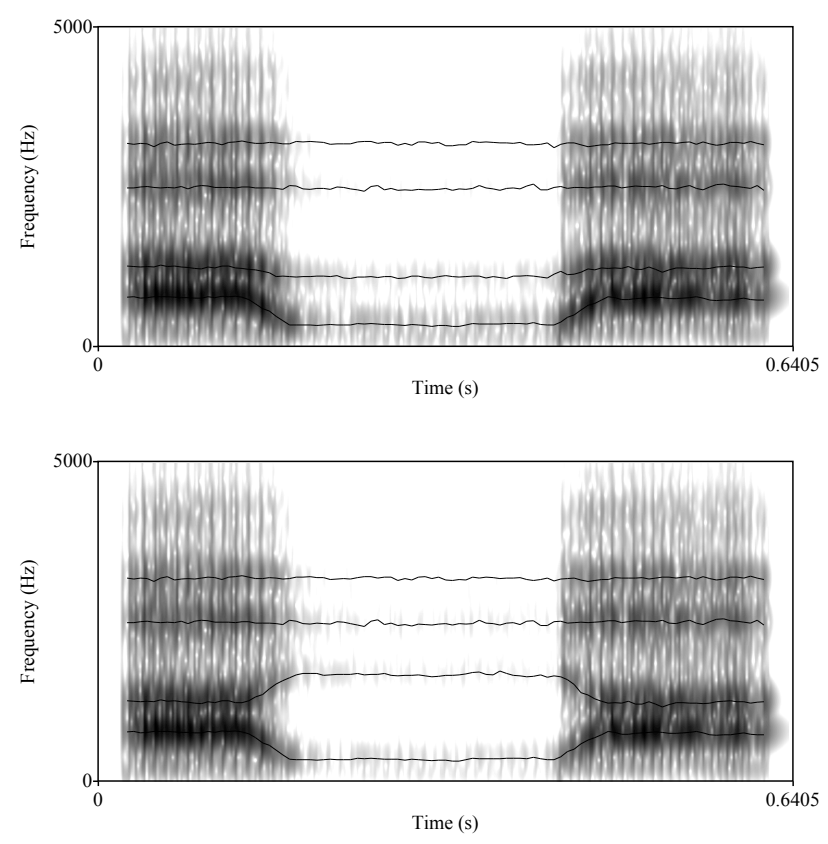

Figure 2.2: Spectrograms for /aba/ (top) and /ada/ (bottom) with formant tracks (synthesized as described in Vroomen, van Linden, Keetels, de Gelder, and Bertelson (2004) and provided by Jean Vroomen). Note the higher second formant (F2) locus for the transitions into and out of the closure for /ada/.

In this paper, we will begin by addressing a very simplified phonetic categorization problem, in which the listener is trying to decide whether a given cue 
value is a $/ \mathrm{b} /$ or $/ \mathrm{d} /$, and later discuss how the approach we develop applies in general. One important cue to this phonetic contrast is the F2 locus, or "target" of the second formant transition (Delattre, Liberman, \& Cooper, 1955). Figure 2.2 shows the spectrograms corresponding to synthesized /aba/ and /ada/ tokens (synthesized by Vroomen, van Linden, Keetels, de Gelder, \& Bertelson, 2004). Figure 2.3 (left panel) shows schematically how the distributions of F2 loci differ for /b/ and /d/: /b/ typically has a lower F2 locus, but there is some variability for both $/ \mathrm{b} /$ and $/ \mathrm{d} /$. There is thus a continuum from $/ \mathrm{b} /$-like to /d/-like F2 locus values.

Let's assume for simplicity's sake that the listener is only considering F2 locus as a cue to the /b/-/d/ contrast. Given an observed F2 locus value, the listener must infer how likely it is that the talker intended to produce the phonetic category $C=/ \mathrm{b} /$. That is, what is the posterior probability $p(C=\mathrm{b} \mid \mathrm{F} 2$ locus $) ?^{4}$ This quantity is found via Bayes rule:

$$
\begin{aligned}
p(\mathrm{~b} \mid \mathrm{F} 2 \text { locus }) & =\frac{p(\mathrm{~F} 2 \text { locus } \mid \mathrm{b}) p(\mathrm{~b})}{p(\mathrm{~F} 2 \text { locus } \mid \mathrm{b}) p(\mathrm{~b})+p(\mathrm{~F} 2 \text { locus } \mid \mathrm{d}) p(\mathrm{~d})} \\
& =\frac{p(\mathrm{~F} 2 \text { locus } \mid \mathrm{b}) p(\mathrm{~b})}{p(\mathrm{~F} 2 \text { locus })} \\
& \propto p(\mathrm{~F} 2 \text { locus } \mid \mathrm{b}) p(\mathrm{~b})
\end{aligned}
$$

Bayes rule captures the fact that the posterior probability depends on three things. First, it depends on the prior probability of the hypothesis, $p(b)$, which could be higher if $/ \mathrm{b} /$ is more frequent in the language than $/ \mathrm{d} /$, or if there are other contextually available sources of information that make /b/ more likely, like lexical, visual, or coarticulatory cues. Second, it depends on the likelihood $p$ (F2 locus $\mid \mathrm{b})$, which is the probability of the observed F2 locus value given that $/ \mathrm{b} /$ was intended by the talker. Finally, it also depends on how credible

\footnotetext{
${ }^{4}$ Here and elsewhere, we write " $\mathrm{b}$ " for "/b/" in equations, for the sake of brevity. We also write $p(\mathrm{~b})$ to indicate $p(C=\mathrm{b})$.
} 
other hypotheses are, which is really a consequence of requiring that the posterior probabilities of all hypotheses add up to one. This is equivalent to the overall probability of the observed F2 locus value, regardless of which hypothesis is true, and since this quantity is the same for all potential hypotheses it is frequently omitted, as in (2.3).

For an ideal listener, the probability of recognizing a /b/ should be the estimate of the posterior probability of $/ \mathrm{b} /$ (and likewise for $/ \mathrm{d} /$ ) (Clayards et al., 2008; Feldman, Griffiths, \& Morgan, 2009). This assumes that the result of speech recognition is not a single category but rather uncertain (or variable) estimates of which categories are more or less likely. This is not a trivial assumption. For example, one might imagine that a listener would improve its categorization accuracy by always 'guessing' the category with the highest probability. However, for speech perception more broadly, there is a benefit to maintaining uncertainty about the category, since additional information often becomes available later in the speech signal (e.g., because of the asynchronous nature of acoustic cues). Indeed, human listeners seem to maintain uncertainty about the speech signal for at least a limited amount of time (cf., right-context effects in word recognition, Bard, Shillcock, \& Altmann, 1988; Connine, Blasko, \& Hall, 1991; Dahan, 2010; Grosjean, 1985)

Treating speech perception as inference under uncertainty provides substantial insight. Much of this comes from the fact that in such a framework, recognition is accomplished not through purely bottom-up template matching but rather by comparing how well each possible higher-level explanation can predict the input signal. This framework provides accounts of effects such as the perceptual magnet effect (Feldman, Griffiths, \& Morgan, 2009), compensation for coarticulation (Sonderegger \& Yu, 2010), and integration of auditory and visual cues (Bejjanki, Clayards, Knill, \& Aslin, 2011). It also describes speech and language processing at other levels, including lexical access (Norris \& Mc- 
Queen, 2008), the incremental integration of words into a syntactic parse (Hale, 2001; Levy, 2008b, 2008a), and pragmatic reasoning (M. C. Frank \& Goodman, 2012; Goodman \& Stuhlmüller, 2013) Moreover, Bayesian inference has been shown to provide a powerful and general computational framework for describing statistically optimal inference under uncertainty, via the integration of prior beliefs and recently observed data. This perspective thus extends to other perceptual and cognitive domains (Griffiths, Chater, Kemp, Perfors, \& Tenenbaum, 2010; Kersten, Mamassian, \& Yuille, 2004; Tenenbaum \& Griffiths, 2001), including sensory adaptation in non-language domains (Fairhall et al., 2001; Körding, Tenenbaum, \& Shadmehr, 2007; Stocker \& Simoncelli, 2006).

One specific advantage of this framework for understanding phonetic adaptation is that it links speech perception behavior with the distribution of cues associated with each category. For an ideal listener, the classification curve for $/ \mathrm{b} /$ and $/ \mathrm{d} /$ responses is derived directly from the respective posterior probabilities (Figure 2.3, left panel), which in turn are computed in part from the corresponding likelihood, or distribution, function for each category:

$$
p(\text { response }=\mathrm{b} \mid \mathrm{F} 2 \text { locus })=p(\mathrm{~b} \mid \mathrm{F} 2 \text { locus }) \propto p(\mathrm{~F} 2 \text { locus } \mid \mathrm{b}) p(\mathrm{~b})
$$

Indeed, listeners do appear to use distributional information in speech perception. Clayards et al. (2008) found that listeners adapt to specific distributions of auditory cues to $/ \mathrm{b} /$ and $/ \mathrm{p} /$. Listeners in this experiment performed a spokenword picture identification task, where some of the stimuli were $/ \mathrm{b} /-/ \mathrm{p} / \mathrm{mini}$ mal pairs like "beach" and "peach". Listeners were randomly assigned to two conditions. In both conditions, the $/ \mathrm{b} /$ and $/ \mathrm{p} /$ percepts were drawn from normal distributions over the primary acoustic cue to the $/ \mathrm{b} /-/ \mathrm{p} /$ contrast (voice onset timing, VOT, Lisker \& Abramson, 1964). In the high-variance condition, the variance around the VOT category means for $/ \mathrm{b} /$ and $/ \mathrm{p} /$ was large; in 
the low-variance condition, it was small. Listeners' classification boundaries reflected the distribution of cues that they experienced: for low-variance exposure, the classification boundaries were steep, while for high-variance exposure the boundaries were shallower, reflecting the greater uncertainty about the intended category that comes with more variable productions of each category. Moreover, the difference in boundary slopes was quantitatively predicted by the difference in the category variances in each case.

This result demonstrates two points. First, by showing that listeners' categorization boundaries reflect the variance of the talker's VOT distributions as predicted by the ideal listener model, they show that listeners are using probabilistic cues in a nearly optimal way. Second, and more importantly for our purposes, they show that listeners are adapting to a change in the statistics of these cues. Because they have no experience with the experimental talker before beginning the experiment, any differences in their classification function after exposure reflects something that they have learned about the talker's VOT distributions over the course of the experiment.

The natural question to ask is: how do listeners get to the point where distributional information is reflected in their behavior? Intuitively, we might say that coming into a new situation-like an experiment-listeners have some beliefs about the distributions of cues for each phonetic category, and that these beliefs change as the listener gains more experience in that situation. These changes in beliefs about how cues are distributed leads to changes in how any given cue is interpreted, resulting in possibly better comprehension or changes in classification behavior. In the next section we show how-like phonetic categorization-this intuitive idea formally corresponds to statistical inference, but at a different level. 


\section{The ideal adapter}

Our ideal adapter framework builds on the ideal listener framework described in the last section. The ideal listener depends on distributional information about each category, in the form of the likelihood function $p(x \mid C)$. We can think of the likelihood function for each category as the listener's prediction about what cue values are likely to occur given that category is produced, and this prediction is used during speech perception to evaluate how well each hypothesized category explains the particular cue value currently being classified. However, we can also think of the likelihood functions as explanations of (and predictions about) the statistics of cues for each category. Crucially, these explanations come from the listener's subjective knowledge of cue distributions, and likely are not exactly identical to the true statistics of cues in the world, because a listener only has finite observations to work with and thus incomplete information about the true distributions. The consequence of this is that the listener has uncertain knowledge of cue distributions.

If the statistics of cues associated with each category were identical or at least similar from one situation to the next, information could be accumulated from all observed values to obtain sufficiently accurate-and certainestimates of the likelihood function. But as discussed above, this is not always the case: talkers can differ dramatically in the acoustic cues they use to realize phonetic categories, and thus the true likelihood function differs across situations (Allen, J. L. Miller, \& DeSteno, 2003; Labov, 1972; Hillenbrand et al., 1995; McMurray \& Jongman, 2011; Pierrehumbert, 2003).

In order to make good use of bottom-up information from acoustic cues, listeners require the appropriate likelihood function for the current situation. Consider again the case of making a /b/-/d/ decision on the basis of the F2 locus cue, but suppose that we have encountered a new talker-call 


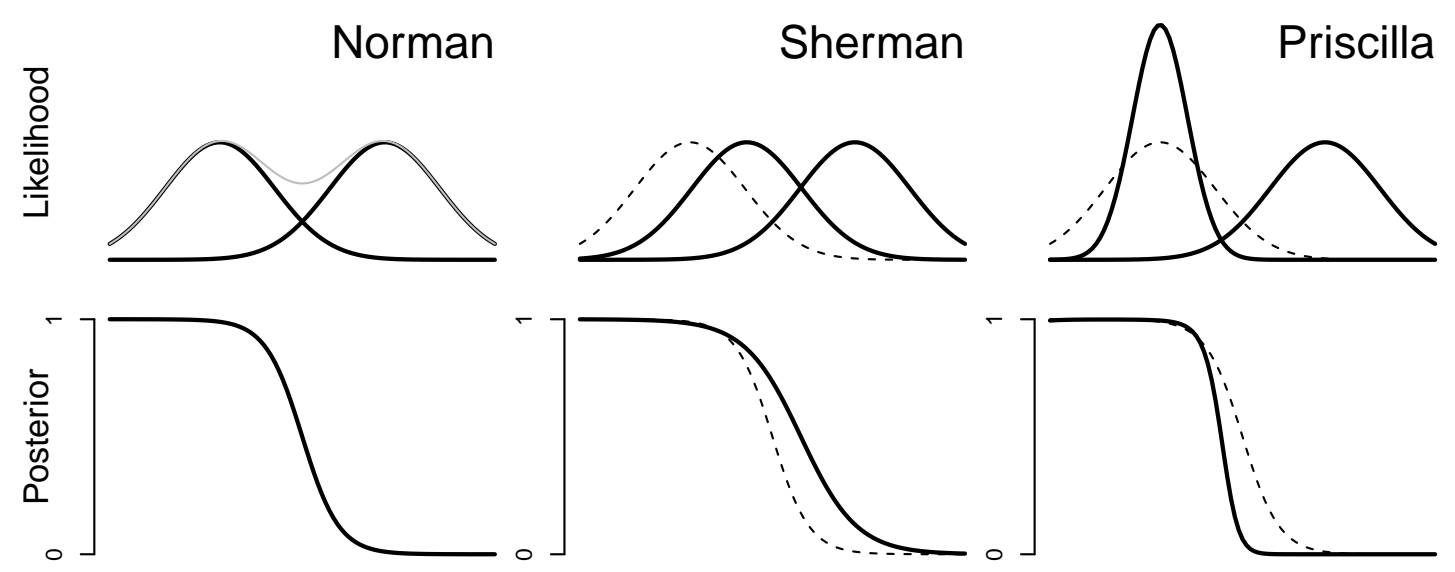

F2 Locus

Figure 2.3: Relationship between F2 locus likelihood functions $p(x \mid c)$ (top) and posterior probability of $/ \mathrm{b} /$, or classification function $p(c \mid x)$ (bottom), for three different talkers: a 'normal' talker (Norman), a 'shifted' talker (Sherman), and a 'precise' talker (Priscilla). Assumes $p(c)=0.5$ for both $c=/ \mathrm{b} /$ and $/ \mathrm{d} /$. Dashed lines show the $/ \mathrm{b} /$ likelihood function and classification function corresponding to the 'normal' talker. Light gray line in top left shows the marginal likelihood, $p(x)=\sum p(x \mid c) p(c)$, which corresponds to the overall distribution of cue values, regardless of which category they came from, and is the sum of the two likelihood functions.

him Sherman-who produces a different distribution of F2 locus values for /b/, a distribution which is shifted to the right (Figure 2.3, middle). If the listener continues to use the likelihood function which matches the 'normal' talker Norman's /b/ distribution, comprehension of Sherman's speech will suffer: cue values which were ambiguous for Norman are now more likely to be generated from /b/ (middle bottom; the ideal classification function for Sherman, the solid line, is above the dashed line). Conversely, cue values that are perfectly ambiguous for Sherman (where the solid line crosses $p(\mathrm{~b} \mid \mathrm{F} 2$ locus $)=0.5)$ would be much more likely to be $/ \mathrm{d} /$ when produced by Norman. That is, a mismatched likelihood function can result in slowed or inaccurate comprehension: inaccurate because the ideal category boundary depends on the likelihood function, and slower because /b/ cue values which are nearly prototypical and highly likely for the new talker would be ambigu- 
ous for the standard talker (the resulting uncertainty slows processing in this sort of task; Clayards et al., 2008; McMurray, Tanenhaus, \& Aslin, 2002).

Similarly, consider the third talker in Figure 2.3 (right), Priscilla, whose /b/ productions are substantially more precise than Norman's, resulting in a lowvariance cue distribution for $/ \mathrm{b} /$, but whose /d/ productions show normal variability. Using Norman's likelihood function to classify Priscilla's productions has similar consequences in this situation: cue values that would have been ambiguous for Norman are now quite a bit more likely to have come from the /d/ distribution because Priscilla's /b/s are so precise.

In both of these situations, comprehension difficulties could be avoided if the listener could use the right likelihood function. If the talker is familiar, this might be as simple as retrieving the right likelihood function based on prior experience with the talker (cf. Goldinger, 1998). ${ }^{5}$ But what if the talker has never been encountered before? This is a distributional learning problem: in order to achieve efficient and accurate comprehension of a novel talker, the listener must learn the cue distributions corresponding to the new talker's phonetic categories. This is similar to the problem faced by an infant acquiring their first language, although the adult listener starts with a substantial amount of prior knowledge. Most notably, they know that there are different phonetic categories for $/ \mathrm{b} /$ and $/ \mathrm{d} /$, and that these categories are generally distinguished by the F2 locus cue. As we discuss in the second half of this article, adult listeners also may have experience with similar talkers, providing them with more or less useful previous experience.

Still, inferring the distributions of F2 locus values corresponding to these

\footnotetext{
${ }^{5}$ There is the additional problem of detecting that the distributions have changed. Change detection in a probabilistic task is a difficult problem that has so far received little attention in research on speech perception and language processing, but has been investigated for other cognitive domains (for a review of the literature, see Qian et al., 2012) and in research on automatic speech recognition (e.g., Ajmera, McCowan, \& Bourlard, 2004; Chen \& Gopalakrishnan, 1998). As we outline in the second half of this article, the framework we propose holds the potential of a unified solution to both adaptation and change detection.
} 
categories is a difficult task, because the inherent variability in these distributions makes each observed cue value ambiguous as evidence for the underlying distribution: if the observed cue value deviates from the listener's predictions, is it due to inherent within-category variance (which will produce some outliers), or is it evidence that the predictions themselves-the likelihood functions-are wrong and need to be updated?

Thus, determining the talker's category distributions is a problem of inference under uncertainty, just like the problem inferring the talker's intended category based on an observed cue value, but at another level. That is, in the same way that the listener can use their knowledge of how well each possible category predicts an observed cue value to infer which category is most likely, they can also use knowledge about how well each possible category distribution predicts the observed statistics of their recent experience in order to infer which underlying distributions are more or less likely in the current situation. The statistically optimal solution to this inference problem can again be described using Bayes Rule. For simplicity's sake, the cue distribution ${ }^{6}$ for a category $c$ can be represented by its mean $\mu_{c}$ and variance $\sigma_{c}^{2}$. Thus the listener's uncertain beliefs about this cue distribution can be represented by a probability distribution over means and variances.

An ideal adapter must infer both the category label $c$ and and the means and variances of the different underlying categories $\mu, \sigma^{2}=\left\{\mu_{c}, \sigma_{c}^{2}\right\}$ (where for our example, $c=/ \mathrm{b} /, / \mathrm{d} /$ ). Formally, this is expressed by the joint posterior distribution over category labels and means and variances, which combines

\footnotetext{
${ }^{6}$ Here we are representing categories as Gaussian (normal) distributions, because they are both mathematically and intuitively tractable. This assumption is not critical for our purposes. The same logic — of belief updating as inferring distributional properties-applies for any parametric or even non-parametric way of representing the distributions.
} 
prior beliefs with the likelihood of the observed evidence: ${ }^{7}$

$$
p\left(c, \mu, \sigma^{2} \mid x\right) \propto \underbrace{p\left(x \mid \mu_{c}, \sigma_{c}^{2}\right)}_{\text {likelihood }} \underbrace{p(c) p\left(\mu, \sigma^{2}\right)}_{\text {prior }}
$$

This captures the fact that after observing a cue value $x$, the listener's joint beliefs about the intended category $C=c$ and the parameters of all categories $\mu, \sigma^{2}$ depend on two things. First, the updated beliefs depend on the likelihood, how well each possible combination of categorization and category parameters can predict the observation $x, p\left(x \mid c, \mu, \sigma^{2}\right)=p\left(x \mid \mu_{c}, \sigma_{c}^{2}\right)$. Second, they depend on the listener's prior beliefs, both about which categories are most likely to be encountered, $p(c)$, and which combinations of category means and variances are most probable, $p\left(\mu, \sigma^{2}\right)$. Both aspects of the prior are based on prior experience. The prior over category probabilities depends on the base rate for each category, as well as its probability in context (based on the surrounding sounds or word, or other acoustic cues besides $x),{ }^{8}$ while the prior over category means and variance depends on the sorts of cue distributions the listener has encountered before and expects to encounter again. The nature of the prior over category means and variances is the focus of Part II below. For now all that matters is that the listener thinks some category means and variances are more likely than others.

The specific way that an ideal adapter updates their beliefs after observing cue value $x$ depends on how they categorize it, and this is captured by the joint posterior distribution $p\left(c, \mu, \sigma^{2} \mid x\right)$. In general, an observation from category $c$ provides the most evidence about the underlying mean and variance of that

\footnotetext{
${ }^{7}$ Using the shorthand notation of $p(c)$ to indicate the probability that the random variable $C$ has value $c$, or $p(C=c)$.

${ }^{8}$ The usefulness of context in providing prior information about phonetic categorization is not limited to situations where the categorization of nearby sounds is known with certainty. Simply knowing that sequences of categories which correspond to actual words are more likely than arbitrary strings provides prior information about how each sound is categorized through the joint distribution of categorizations $p\left(\ldots, c_{i-2}, c_{i-1}, c_{i}, c_{i+1}, \ldots\right)$.
} 
category. In the case where the prior beliefs about the parameters of each category are independent of each other, $p\left(\mu, \sigma^{2}\right)=\prod_{c} p\left(\mu_{c}, \sigma_{c}^{2}\right)$, the beliefs about category $c_{i}$ are only updated if the observation is classified as $C=c_{i}$.

$$
p\left(\mu_{c_{i}}, \sigma_{c_{i}}^{2} \mid x, C\right) \propto \begin{cases}p\left(x \mid \mu_{c_{i}}, \sigma_{c_{i}}^{2}\right) p\left(\mu_{c_{i}}, \sigma_{c_{i}}^{2}\right) & \text { if } C=c_{i} \\ p\left(\mu_{c_{i}}, \sigma_{c_{i}}^{2}\right) & \text { if } C \neq c_{i}\end{cases}
$$

In cases where there is uncertainty about how the observation $x$ should be categorized, an ideal adapter should update the beliefs about category $c_{i}$ as a mixture of the updated beliefs under each possible categorization, weighted by how likely that categorization is overall (averaging or marginalizing over current category parameter beliefs):

$$
p\left(\mu_{c_{i}}, \sigma_{c_{i}}^{2} \mid x\right)=\sum_{c} p\left(\mu_{c_{i^{\prime}}} \sigma_{c_{i}}^{2} \mid x, C=c\right) p(C=c \mid x)
$$

Again, if we assume that the beliefs about different categories are independent, this mixture consists of two components: one where $x$ is categorized as $C=c_{i}$ and beliefs about category $c_{i}$ are updated, and one where it is not and no belief updating occurs: ${ }^{9}$

$$
\begin{aligned}
p\left(\mu_{c_{i}}, \sigma_{c_{i}}^{2} \mid x\right)= & p\left(\mu_{c_{i}}, \sigma_{c_{i}}^{2} \mid x, C=c_{i}\right) p\left(C=c_{i} \mid x\right) \\
& +p\left(\mu_{c_{i}}, \sigma_{c_{i}}^{2}\right) p\left(C \neq c_{i} \mid x\right)
\end{aligned}
$$

To return to the example above of classifying a token as either /b/ or /d/ based on F2 locus, the posterior distribution over the mean and variance of /b/

\footnotetext{
${ }^{9}$ In the general case where the prior beliefs about different categories' parameters are not independent, the posterior is still a mixture, but is a mixture of beliefs, updated in different ways and to different extents, rather than just updated and non-updated beliefs.
} 
after observing a particular F2 locus value is thus

$$
\begin{aligned}
p\left(\mu_{\mathrm{b}}, \sigma_{\mathrm{b}}^{2} \mid \mathrm{F} 2 \text { locus }\right)= & p\left(\mu_{\mathrm{b}}, \sigma_{\mathrm{b}}^{2} \mid \mathrm{F} 2 \text { locus }, \mathrm{b}\right) p(\mathrm{~b} \mid \mathrm{F} 2 \text { locus }) \\
& +p\left(\mu_{\mathrm{b}}, \sigma_{\mathrm{b}}^{2}\right) p(\mathrm{~d} \mid \mathrm{F} 2 \text { locus })
\end{aligned}
$$

In conversational speech, acoustic observations are often labeled with high certainty, and so $p\left(C=c_{i} \mid x\right) \approx 1$ for some category $c_{i}$. Such label information can come both from top-down linguistic context (like phonotactics or lexical disambiguation), or from other bottom-up cues. For example, when distinguishing /b/ from /d/, the closure of the lips during /b/ provides a very informative visual cue, effectively labeling the auditory percept (Vroomen, van Linden, Keetels, et al., 2004).

In such cases where there is some other source of information that labels the observed F2 locus value as a /b/, the resulting conditional posterior distribution over the mean and variance of $/ \mathrm{b} /$ simplifies to:

$$
p\left(\mu_{\mathrm{b}}, \sigma_{\mathrm{b}}^{2} \mid \mathrm{F} 2 \text { locus, } \mathrm{b}\right) \propto \underbrace{p\left(\mathrm{~F} 2 \text { locus } \mid \mathrm{b}, \mu_{\mathrm{b}}, \sigma_{\mathrm{b}}^{2}\right)}_{\text {likelihood }} \underbrace{p\left(\mu_{\mathrm{b}}, \sigma_{\mathrm{b}}^{2}\right)}_{\text {prior beliefs }}
$$

Here, the relevant prior distribution is just the listener's prior beliefs about the mean and variance of the F2 locus cue for the /b/ category. Likewise, the likelihood considers only how well each combination of /b/ mean and variance account for the observed cue value. Below, we model incremental adaptation for cases where the category labels are known with high certainty (and thus (2.8) holds). We also assume that the prior beliefs about / b/ and /d/ are independent. We make these assumptions for the sake of simplicity and tractability in modeling, and it is important to keep in mind that they do not represent assumptions of the framework, which makes qualitatively the same predictions whether or not these assumptions turn out to be true. 
In sum, the ideal adapter framework predicts that optimal phonetic adaptation depends on three things: the statistics of the observed percepts (e.g. their mean and variance), the listener's prior beliefs about the statistics of the relevant categories, and the listener's belief that there is a need to adapt (including their beliefs about the amount of variation in the relevant category across talkers and situations). In the next five sections, we illustrate the role of the first two factors in phonetic adaptation experiments where the third factor is a given (i.e., where there is a clear need to adapt and for which previous work has shown that listeners indeed adapt, Bertelson et al., 2003; Kraljic \& Samuel, 2005; Norris et al., 2003). In order to do this we specify a basic belief updating model in the ideal adapter framework which quantifies how the exposure statistics and the listener's prior beliefs about those statistics interact.

With this model in hand, we do five things. First, as a basic evaluation we address the phenomenon of phonetic recalibration or perceptual learning. Such perceptual learning is typically thought to be due to changes in the underlying representations of the adapted categories which generally serves the purpose of robust speech perception, and is naturally accounted for by the ideal adapter framework. In particular, we show that the incremental build-up of recalibration is accounted for by our basic belief updating model.

Second, we illustrate how the way in which the model accounts for the build-up of recalibration potentially sheds light on the underlying processes. Specifically, the model captures the fact that recalibration is often ambiguous between a change in the underlying mean of the category versus a relaxation of the criterion for what counts as an acceptable exemplar, or a change in the variance of the category.

Third, we examine the predictions of this framework for the selective adaptation paradigm, a paradigm which is typically not considered to be due to the perceptual learning which serves robust speech perception. However, we 
show that the belief updating model accounts for the incremental build-up of this phenomenon as well, using very similar parameters as for recalibration.

Fourth, we explore a little studied property of phonetic recalibration that, prima facie, would seem to stand in conflict with our hypothesis that adaptation serves robust speech perception: prolonged, repeated exposure to the exact same stimulus can eventually undo the recalibration effect (Vroomen, van Linden, de Gelder, \& Bertelson, 2007). However, we show that not only is this predicted by the ideal adapter framework under a range of conditions, the belief updating model which accounts for the build-up of selective adaptation and recalibration also accounts for the effect of prolonged repeated exposure in each, and does so simultaneously with a single set of parameters.

Fifth, motivated by the potential link between selective adaptation and recalibration suggested by the proposed framework, we present novel data from a web-based perception experiment which tests the predictive power of our model. Specifically, we test adaptation conditions that are intermediate between recalibration and selective adaptation, for which the model predicts a continuum between classic recalibration and selective adaptation responses.

\subsubsection{Basic evaluation of the ideal adapter framework: pho- netic recalibration}

We begin with an illustration of the basic mechanics of the ideal adapter framework. For this we focus on experiments in which listeners are exposed to a novel talker with an unusual realization of a phonetic contrast. There is a great deal of evidence about the results of incremental adaptation. Much of it comes from studies of "phonetic recalibration", or "perceptual learning" (Bertelson et al., 2003; Kraljic \& Samuel, 2005; Norris et al., 2003). These studies use a continuum between two sounds, generally constructed by interpolating between 
prototypical endpoint tokens (e.g. Kraljic \& Samuel, 2005; Norris et al., 2003) or by parametrically manipulating a critical acoustic cue which distinguishes between the two categories. For instance, a /b/-/d/ continuum might be constructed by manipulating F2 locus, as described above (Bertelson et al., 2003; Vroomen, van Linden, Keetels, et al., 2004). During an exposure phase, listeners hear the item from this continuum which is most ambiguous between /b/ and /d/. This auditorily ambiguous segment is paired with information which consistently "labels" or disambiguates it as a /b/. This labeling is achieved via, for example, lexical disambiguation (e.g. replacing the $/ \mathrm{b} /$ in $c l u b$ with the ambiguous segment, Kraljic \& Samuel, 2005; Norris et al., 2003, etc.) or visual disambiguation (pairing the auditorily ambiguous sound with a video of a person articulating a /b/, which results in a visible labial closure unlike articulation of $/ \mathrm{d} /$ ). After exposure, changes to the listener's classification function are assessed, for example, by means of a classification test over unlabeled sounds drawn from the continuum (e.g. classifying a continuum of sounds from a prototypical /aba/ to a prototypical /ada/ without either lexical or visual disambiguation).

In what follows, we use the notation $x_{c_{1} c_{2}}$ to refer to a sound that is auditorily ambiguous between categories $c_{1}$ and $c_{2}$, and use superscripts to refer to labeled sounds. So, for example, $x_{\mathrm{bd}}^{\mathrm{b}}$ is a sound auditorily ambiguous between /b/ and /d/ which is labeled (disambiguated) as /b/.

Perceptual recalibration rapidly results in shifted category boundaries. For example, after as few as 10 exposures to $x_{\mathrm{bd}}^{\mathrm{b}}$, the /b/ category has 'grown': more of the continuum is now classified as $/ \mathrm{b} /$, when tested without the labeling information (Vroomen, van Linden, de Gelder, \& Bertelson, 2007). The opposite shift is observed for exposure to $x_{\text {bd }}^{\mathrm{d}}$. This is illustrated schematically in Figure 2.4 (A). Similarly rapid perceptual recalibration has been observed along a variety of phonetic contrasts, including vowels (Maye, Aslin, \& Tanen- 

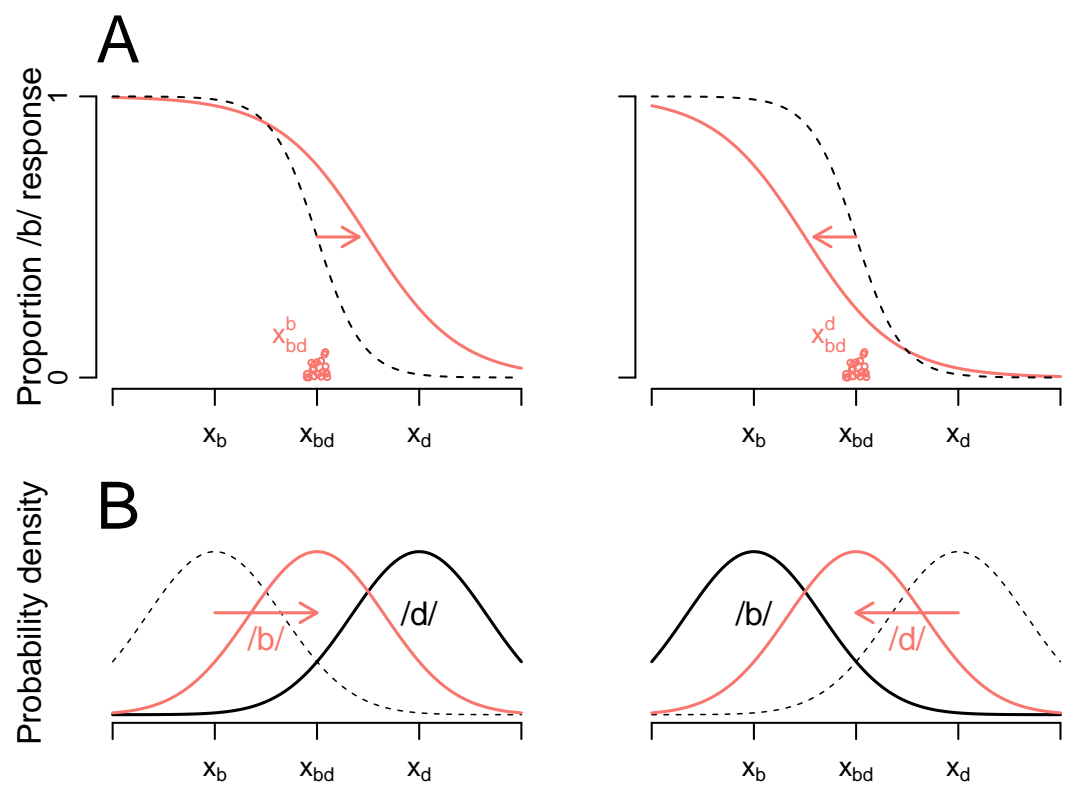

Figure 2.4: Schematic illustration of the results of perceptual recalibration on classification of a /b/-/d/ continuum (A), and the changes in the listener's beliefs about the underlying distributions which we propose to account for the changes in classification (B). Dashed lines show pre-exposure classification functions and distributions, while solid lines show post-recalibration. Left panels show the results of exposure to $x_{\mathrm{bd}}^{\mathrm{b}}$, and the right to $x_{\mathrm{bd}}^{\mathrm{d}}$.

haus, 2008), fricative place of articulation and manner (Kraljic \& Samuel, 2005;

Norris et al., 2003), and stop consonant place (Bertelson et al., 2003) and voicing (Kraljic \& Samuel, 2006). Perceptual recalibration is typically investigated under the assumption that it reflects the same processes that support general accent adaptation. This assumption is not trivial but there is some support that perceptual recalibration is not simply an artifact of the stimuli being presented in isolation (Eisner \& McQueen, 2006) or there only being one unusual pronunciation (Reinisch \& Holt, 2014). We return to these issues in the next section.

Qualitatively, perceptual recalibration exhibits several properties that are expected under the ideal adapter framework. First, recalibration seems to reflect implicit learning over phonetic contrasts, rather than strategic effects such as response bias (Clarke-Davidson, Luce, \& Sawusch, 2008), or weakening of 
the criterion for what counts as an acceptable example of a category (Maye, Aslin, \& Tanenhaus, 2008, but see next section). Recalibration also appears to affect speech perception through changes in sublexical phonetic category representations since perceptual recalibration effects generalize to novel words by the same talker containing the recalibrated segment (McQueen, Cutler, \& Norris, 2006).

Second, perceptual recalibration seems to last: when listeners classify tokens from the same talker 12 hours after initial testing, the magnitude of adaptation is the same as right after initial exposure (Eisner \& McQueen, 2006). As we discuss in more detail in the second part of this article, longevity of changes in category boundaries (for a particular situation) is expected under our proposal that adaptation serves to make speech perception robust to changes in situation.

More specifically, the qualitative changes in classification boundaries observed during perceptual recalibration is naturally predicted by the ideal adapter framework (Figure 2.4, B). Take, for example, the case where the listener is exposed to $x_{\mathrm{bd}}^{\mathrm{b}}$ (Figure 2.4, left). As the listener updates their beliefs about the shifted distribution of cues for $/ \mathrm{b} /$, shifting the mean towards the observed cue values, stimuli in the middle of the /b/-/d/ continuum which previously had roughly equal likelihood under either category (and thus are sometimes perceived as $/ \mathrm{b} /$ and sometimes as $/ \mathrm{d} /$ ) are now more likely to have resulted from $/ \mathrm{b} /$, resulting in more $/ \mathrm{b} /$ responses to unlabeled test stimuli, especially in the previously-ambiguous region of the continuum.

\section{Incremental recalibration}

It is encouraging that the ideal adapter framework provides a qualitative account of the results of recalibration. But can this framework account for incremental changes in behavior? Belief updating is an incremental process, where 
the listener accumulates information about the talker's cue distributions one observation at a time. The ideal adapter framework thus not only predicts asymptotic classification behavior-after the listener has fully adapted to the talker's cue distributions-but also how their classification behavior changes with each additional piece of evidence. Unfortunately, few studies have investigated the incremental effects of exposure to a novel distribution of sounds, such as would be typical for a new talkers.

A notable exception is Vroomen, van Linden, de Gelder, and Bertelson (2007). Listeners in their study were exposed to repetitions of an audio-visual adaptor, which was composed of a video recording of a talker articulating either /aba/ or /ada/, dubbed with an audio item from a 9-item, synthetic /aba/ $\left(x_{\mathrm{b}}=1\right)$ to /ada/ $\left(x_{\mathrm{d}}=9\right)$ continuum. The audio component for each participant was the continuum item that was most ambiguous, $x_{\mathrm{bd}}$. The most ambiguous item was determined during a pre-test block of 98 trials where the entire /aba/-/ada/ continuum was classified. ${ }^{10}$

Instead of the typical recalibration procedure, where exposure and test are separated, Vroomen, van Linden, de Gelder, and Bertelson (2007) measured the degree and direction of adaptation by interspersing audio-only test blocks throughout each exposure block, after 1, 2, 4, 8, 16, 32, 64, 128, and $256 \mathrm{cu}$ mulative exposures to the audio-visual adaptor. Specifically, they measured the average proportion of $/ \mathrm{b} /$ responses to six-trial test blocks (the three most ambiguous items $\left\{x_{\mathrm{bd}}-1, x_{\mathrm{bd}}, x_{\mathrm{bd}}+1\right\}$ each repeated twice).

Each participant completed sixteen exposure blocks of 256 exposures. Half of the exposure blocks used a / b/ audio-visual stimulus for exposure, and the other half used a /d/. Of the /b/-exposure blocks, half of these used the auditorily ambiguous stimulus as described above, while the other half used the

\footnotetext{
${ }^{10}$ There were 14 repetitions of each stimulus, except for the two endpoints $x=1$ and $x=9$ which were repeated only 6 times and the next most prototypical items $x=2$ and $x=8$ which were repeated 8 times each
} 
prototypical /b/ endpoint of the acoustic continuum $(x=1)$, and likewise for the /d/-exposure blocks. ${ }^{11}$ Because our goal is to illustrate the workings of the ideal adapter framework when the listener has little prior experience that might be relevant for the current situation, we focus on the first 64 exposures of the first block from each participant (we return to the issue of extended exposure below).
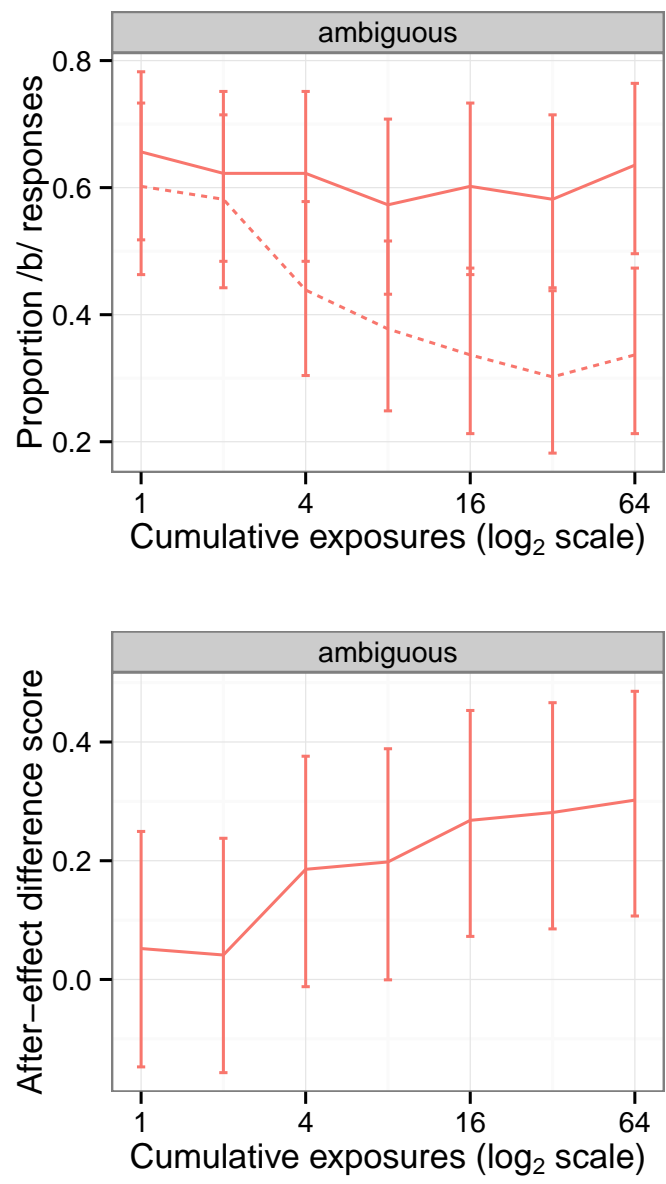

Figure 2.5: Recalibration results from Vroomen, van Linden, de Gelder, and Bertelson (2007), showing both the proportion of /b/ responses (top, solid line $/ \mathrm{b} /$ exposure and dashed line /d/ exposure) and the aftereffect difference score (bottom) for the first 64 critical exposures in the first exposure block. Error bars indicate $95 \%$ confidence intervals.

\footnotetext{
${ }^{11}$ The prototypical exposure blocks are discussed later.
} 
Figure 2.5 shows the results of Vroomen, van Linden, de Gelder, and Bertelson (2007) demonstrating the build-up of recalibration in the first 64 critical exposures in the first exposure block. The top panel shows the proportion /b/ responses for $x_{\mathrm{bd}}^{\mathrm{b}}$ and $x_{\mathrm{bd}}^{\mathrm{d}}$ adaptors separately; more /b/ responses after /b/ exposure (solid line) indicates recalibration, and vice-versa for $/ \mathrm{d} /$ exposure (dashed line). A natural measure of the degree of recalibration is thus the aftereffect difference score between $/ \mathrm{b} /$ and $/ \mathrm{d} /$ exposure. A positive aftereffect indicates that /b/ exposure increased /b/ responses, and /d/ exposure decreased /b/ responses (and increased /d/ responses), which corresponds to recalibration (Figure 2.5, bottom).

Recalibration builds up rapidly but incrementally over the first 64 exposures. As discussed above, this build up follows naturally from the ideal adapter framework, as each exposure to the auditorily ambiguous adaptor contributes to a shift in the listener's estimate of the category mean. Next, we quantify and test this prediction.

\section{Modeling build-up of recalibration}

In order to evaluate the ability of the ideal adapter framework to account for the results of Vroomen, van Linden, de Gelder, and Bertelson (2007) on the incremental build-up of recalibration, we implemented a basic Bayesian belief updating model based on the principles of the ideal adapter framework.

Bayesian belief updating model We used a mixture of Gaussians as the underlying model of phonetic categories, where each phonetic category $c \in\{b, d\}$ corresponds to a normal distribution over cue values $x$ with mean $\mu_{c}$ and variance $\sigma_{c}^{2}$ (e.g. Figure 2.3, top). Thus, the likelihood of observation $x$ under category $c$ is

$$
p\left(x_{i} \mid c, \mu, \sigma^{2}\right)=\operatorname{Normal}\left(\mu_{c}, \sigma_{c}^{2}\right)
$$


The listener's uncertain beliefs about phonetic categories are captured by additionally assigning probability distributions to the means $\mu_{c}$ and variances $\sigma_{c}^{2}$ of each phonetic category. The prior distribution $p\left(\mu_{c}, \sigma_{c}^{2}\right)$ represents the listener's beliefs about category $c$ before exposure to the experimental stimuli, and the posterior $p\left(\mu_{c}, \sigma_{c}^{2} \mid X\right)$ captures the listener's beliefs after exposure to stimuli $X=\left(x_{1}, x_{2}, \ldots, x_{N}\right)$ which are known to come from category $c$ (which means that the category labels $C=c$ are known). These two distributions are related via Bayes' Rule:

$$
p\left(\mu_{c}, \sigma_{c}^{2} \mid X, C=c\right) \propto p\left(X \mid \mu_{c}, \sigma_{c}^{2}, C=c\right) p\left(\mu_{c}, \sigma_{c}^{2}\right)
$$

We used a conjugate prior for the Normal distribution with unknown mean and variance (Appendix A.1), and this prior distribution has two types of hyperparameters. ${ }^{12}$ The first set of hyperparameters captures the prior expected values of the means and variances. The second set of hyperparameters captures the degree of confidence, or, conversely, uncertainty associated with the prior beliefs. Put differently, they determine how much current observations are weighted against previous experience in determining beliefs about the category distributions. In this model, there are two different degrees of confidence: one for the category means, denoted $\kappa_{0}$, and the other for the category variances, denoted $v_{0}$ (see the Appendix for details). An intuitive interpretation of these hyperparameters is as the effective sample size of the prior beliefs. For instance, if the category mean confidence parameter is $\kappa_{0}=10$, then after ten new observations the listener's beliefs about the category mean to equally reflect previous and current experience. With fewer than ten new observations, the listener's beliefs about the category mean will be dominated by the mean

\footnotetext{
${ }^{12}$ We use the term hyperparameters for terminological clarity to distinguish the model parameters from the category distribution parameters-means and variances-whose prior and posterior distributions are defined by the model parameters, the hyperparameters.
} 
expected based on previous experience; with more than ten new observations the beliefs about the mean will be increasingly dominated by the mean of the new observations. These hyperparameters thus capture the gradient trade-off between prior experience and current experience in determining the listener's beliefs about phonetic categories. They can be thought of as pseudocounts or the number of prior experiences that are relevant for the current situation. ${ }^{13}$

Finally, it is not a priori obvious whether adaptation occurs at the level of auditory cues individually or at some higher level where information is integrated from multiple auditory and/or visual cues. Thus the model includes a third hyperparameter, $w$ which determines the weight given to the visual cue value in determining the percept. This hyperparameter ranges from $w=0$ (perceived cue value is not influenced by the visual cue) and $w=1$ (perceived cue value entirely determined by the visual cue). Adaptation over integrated cues might arise because the listener attempts to infer the talker's intended production based on multiple partially informative cues (including top-down category distribution information, Feldman, Griffiths, \& Morgan, 2009). For more discussion, refer to the Appendix.

Model fitting The hyperparameters were fit in a two-step process, which is described in detail in the Appendix. The first step is to fix the expected prior means and variances based on the classification curves measured during pretest, before exposure to the audiovisual adaptor. These hyperparameters are thus not free parameters of the model, in that they are not adjusted to improve the fit to the actual adaptation test data.

The second step is to estimate the three free hyperparameters (i.e., the effective prior sample sizes $v_{0}$ and $\kappa_{0}$ and the visual cue weight $w$ ) based on the

\footnotetext{
${ }^{13}$ Even though comparable fits can be obtained using a single, overall effective prior sample sizes (e.g. $\kappa_{0}=v_{0}$ ), the two were fit as separate in order to evaluate the extent to which recalibration was primarily driven by a shift in the category mean or a change in variance.
} 
actual adaptation data. The posterior distribution of the free hyperparameters was obtained using MCMC sampling with a weakly informative prior (to ensure a proper posterior, Gelman, Carlin, Stern, \& Rubin, 2003). For further details, we refer to Appendix A.1. Because of the limited amount of data from each participant (only six trials per test block), we chose to fit the model to the aggregate data from all participants (see Appendix A.3 for motivation).

\section{Results and discussion}

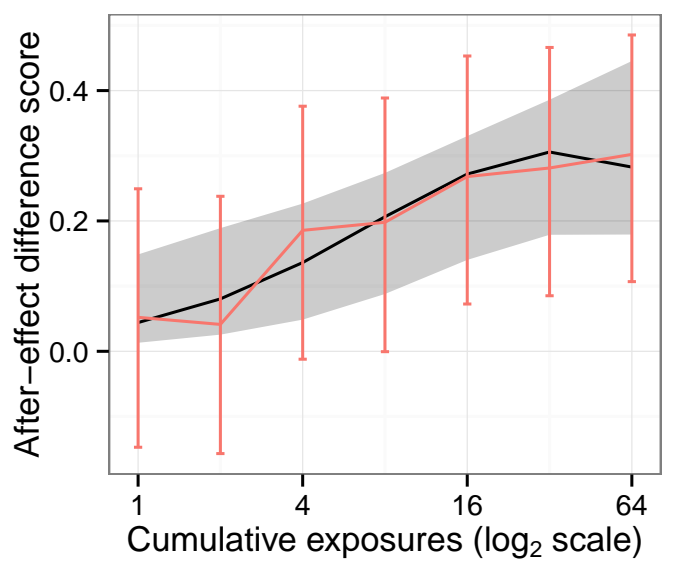

Figure 2.6: Belief updating model fit to build-up of recalibration data from Vroomen, van Linden, de Gelder, and Bertelson (2007). The $x$-axis shows the number of cumulative exposures to the adaptor (on a log scale), and the $y$ axis shows the aftereffect difference score. The solid black line shows the MAP (maximum a posteriori) estimate predictions $\left(r^{2}=0.96\right)$. The error bars and shaded region show $95 \%$ credible intervals for the data and model predictions, respectively (see Appendix A.3.3).

The model's fit against the data is shown in Figure 2.6. The predicted responses are plotted on the aftereffect scale for better comparison to Vroomen, van Linden, de Gelder, and Bertelson (2007). The model effectively captures the qualitative fact that recalibration leads to positive aftereffects which build up incrementally, and provides a quantitatively good fit as well $\left(r^{2}=0.96\right)$. 
Specifically, the model captures the fact that recalibration starts off relatively weak and gradually becomes stronger, before eventually leveling off. Thus, not only is the qualitative result of recalibration-a positive aftereffect-predicted by the ideal adapter framework, the effect of cumulative exposure on the incremental build-up of the effect is also predicted well. This suggests that listeners incrementally integrate each observed cue value with their prior beliefs in a way that is predicted by the ideal adapter framework.

We can draw a number of conclusions from the values of the hyperparameters themselves. The best-fitting hyperparameter values are $v_{0}=71, \kappa_{0}=11$, and $w=0.53$. First, relative to the real overall sample size-the number of $/ \mathrm{b} / \mathrm{s}$ and $/ \mathrm{d} / \mathrm{s}$ encountered in the world by a typical English-speaking adult-the best-fitting effective prior sample sizes are extremely low. That is, listeners appear to put very little weight on their prior beliefs, adapting very quickly to the shifted cue distribution that they observe while taking slightly longer to adapt to the tight clustering (low variance). This may be surprising at first glance, but it is actually qualitatively predicted by the ideal adapter framework. In the ideal adapter framework, whether or not (and how much) a listener adapts depends on how relevant they think their previous experiences are for the current situation. Thus in situations like a recalibration experiment where listeners encounter odd-sounding, often synthesized speech in a laboratory setting, they may have little confidence, a priori, that any of their previous experiences are directly informative. We discuss this point in length in the second half of this paper, where we elaborate on the crucial role of prior experiences for robust speech perception.

Second, the best-fitting value of the visual cue weight hyperparameter $w$ places approximately equal weight on the audio and visual cue values. This means that, according to the best-fitting model, listeners perceive the cue value as not fully ambiguous. This makes an interesting prediction about the effects 
of extended exposure to the same stimulus that we return to below.

Third, the joint distribution-rather then just the point estimates-of the prior effective sample size hyperparameters (Figure 2.7) reveal that as long as one of these hyperparameters is low-on the same order of magnitude of the number of exposures to the adaptor stimulus-the other confidence hyperparameter can become extremely large and not change the model's predictions so much that the likelihood suffers. This is because there are two ways that the positive aftereffect observed here (and in other recalibration studies) might come about after exposure to an ambiguous adaptor stimulus. This is discussed in more detail next.

\subsubsection{Recalibration by category shift or expansion?}

One of the advantages of model-fitting using Bayesian methods is that it allows us to evaluate the range of model hyperparameters which provide a good fit to the data. For the build-up of recalibration modeled in the previous section, the full posterior distribution over model hyperparameters (effective prior sample sizes and visual cue weight) provides interesting insight into how a human learner might adapt. To illustrate this, we examine the posterior distribution of the prior effective sample sizes for the category means and variances given the behavioral data.

The joint distribution of the two confidence hyperparameters-the mean confidence $\kappa_{0}$ and the variance confidence $v_{0}$-is shown in Figure 2.7. This distribution covers an extremely wide range of both hyperparameters, although this entire range still results in qualitatively consistent predictions about the aftereffect at each level of exposure (Figure 2.6). The wide range covered by the posterior distribution of hyperparameters is due to the limited amount of data available to the model in this particular case. Note that this is not a problem. It 


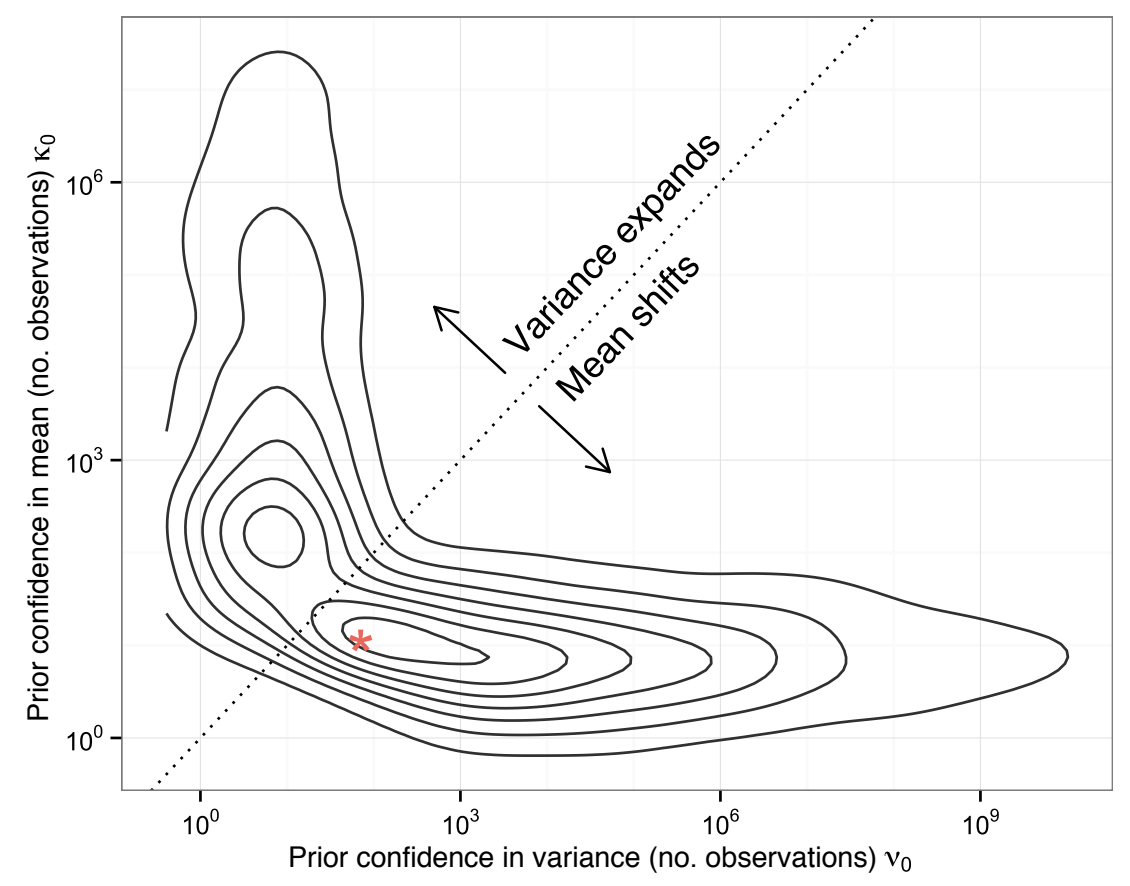

Figure 2.7: The belief-updating model finds two different ways of fitting the build-up of recalibration (Figure 2.6), as illustrated by this density plot of the distribution of the mean and variance prior confidence parameters $\left(\kappa_{0}\right.$ and $v_{0}$, respectively) that are consistent with the data (estimated by samples via MCMC). The diagonal shows solutions with equal confidence in prior beliefs about the mean and variance. Points below the line have higher confidence in the variance, and adapt by shifting the category mean. Points above the line have higher confidence in the mean and adapt by expanding the category variance. Note that even though the best-fitting parameters (red asterisk, and curve in Figure 2.6) are below the line (and shift the mean), there are areas of high posterior probability on both sides of the line (hills on the contour plot).

merely reflects that these data do not uniquely constrain the model. A human learner exposed to the same data would face the same problem. Indeed, we will see below that when the model is constrained by further data, the posterior distribution of the hyperparameters will become more narrow.

Of interest is that the posterior is bimodal: there are two ways that belief updating can account for the build-up of recalibration. The best-fitting (MAPestimate) hyperparameters correspond to a shift in the mean of the adapted 
category: the prior effective sample size of the mean, $\kappa_{0}$, is less than that of the variance, $v_{0}$, and as a result the prior beliefs about the mean are overcome more quickly than the variance. However, this pattern is only true for roughly half the samples from the joint posterior of the hyperparameters $\left(p_{\mathrm{MCMC}}\left(v_{0}>\right.\right.$ $\left.\left.\kappa_{0}\right)=0.55\right)$. In the other half of the samples, the prior effective sample size for the mean is on average very high, meaning that the positive aftereffect observed in the data is modeled as a change in the variance of the adapted category, with a mean that is essentially fixed.

This combination of hyperparameters-flexible variance and fixed meancan lead to a positive aftereffect after exposure to auditorily ambiguous but labeled tokens in the following way. If the listener has very high confidence in the mean of each category coming into a new situation, then repeated exposure to an ambiguous segment which is labeled as belonging to one category is best explained by the hypothesis that the talker is producing that category with a high degree of variability. Increasing the variance of the recalibrated category in this way means that more likelihood is assigned by that category to the previously-ambiguous part of the continuum to the recalibrated category, and thus leads to a positive aftereffect.

Thus, a positive aftereffect is qualitatively consistent with both a shift in mean and an increase in category variance. Moreover, the joint distribution of hyperparameters fit to the build-up of recalibration observed by Vroomen, van Linden, de Gelder, and Bertelson (2007) show that in the ideal adapter framework, the quantitative effect of cumulative exposure on the build-up of recalibration is also ambiguous in the same way.

Maye, Aslin, and Tanenhaus (2008) behaviorally investigated a similar question. Specifically, they wondered whether positive aftereffects typically observed in recalibration experiments were really due to a shift in the underlying category, or just a relaxation of the criterion for what counts as a good 
exemplar of the adapted category. They exposed listeners to vowels that were shifted in a particular direction (e.g. shifting the high vowel /I/ in wicked down to the mid vowel $/ \varepsilon /$ to make 'wecked'). In a lexical decision task after exposure to such downward shifts, listeners accepted more nonwords that were downward-shifted versions of real words, but not nonwords that were upward-shifted words. This corresponds, in the ideal adapter framework, to a shift in the means of the adapted categories, without a substantial change in the variance.

It may be tempting to conclude based on these results that all recalibration effects result from shifting category means. However, the ideal adapter framework predicts that positive aftereffects due to changes in either means and variances are possible, in different situations, especially depending on whether the listener has greater confidence in their prior beliefs about category variances or means. This is, to the best of our knowledge, a novel prediction. Since one of our goals is to provide a guiding framework for future work on speech processing and adaptation, we elaborate on this prediction.

When might the listener have greater confidence in the mean of a category rather than its variance, and vice versa? In the ideal adapter framework, the listener's prior beliefs about a category parameter constitute a prediction about the distribution of values they might expect that parameter to take on in the future. The level of confidence in prior beliefs is closely related to the level of variability of a particular category parameter that the listener expects across situations. For cues whose typical values vary across situations (e.g. formant frequencies), an ideal adapter should expect substantial variability in the underlying means, in order to be prepared to shift their beliefs about category means on that cue when appropriate.

The variance of a particular cue for a particular category is closely related to how reliable that cue is at distinguishing one category from another (Allen \& 
J. L. Miller, 2004; Clayards et al., 2008; Newman et al., 2001; Toscano \& McMurray, 2010): for two categories with fixed means, increasing the variance of both categories means that their distributions will overlap more and, on average, observing that particular cue will be less informative about the intended category. Thus for a cue which varies in reliability from one situation to the next (with relatively stable category means), the ideal adapter should in general be more likely to adjust category variance than means. ${ }^{14}$

Thus, the ideal adapter framework predicts that there are range of strategies available to the listener for adapting to new talkers. In real-life accent adaptation, there are usually many categories and cue dimensions where an accent is unusual, and in some cases these differences can be due to both changes in the cue values typically used to realize a category (the mean) and changes in how reliable a given cue is at distinguishing a category (the variance). In real speech there are many partially informative cues to any given category, and it may be a completely reasonable strategy for the listener to simply decide that a particular cue is uninformative and ignore it (or at least downweight it).

This points to a critical empirical gap in our understanding of speech perception. Most existing work on phonetic adaptation follows one of two approaches. The first approach emphasizes relatively natural conditions and accent variability, where language occurs in context (e.g. sentences) and listeners must adapt to accents that vary along many categories and cue dimensions (Baese-berk et al., 2013; Bradlow \& Bent, 2008; Clarke-Davidson \& Garrett, 2004; Sidaras, Alexander, \& Nygaard, 2009). The second approach is that of perceptual recalibration/learning, which typically presents speech as isolated words or syllables and emphasizes acoustic manipulation of a single category

\footnotetext{
${ }^{14}$ This is not to say that an ideal adapter would not adapt to changes in category distributions for cues whose means or variances do not vary much across situations. Rather, the amount of variability in a particular category's statistics over situations combines with the listener's overall level of confidence that their prior experience is relevant for the current situation.
} 
or auditory cue. While perceptual recalibration has been observed when these unusual pronunciations were presented as part of running speech in a story (Eisner \& McQueen, 2006) or as words spoken by a talker who has a real foreign accent (Reinisch \& Holt, 2014), it is an open question under what conditions listeners downweight cues and when they track changes in mean cue values during naturalistic accent adaptation.

In order to address listeners' ability to adapt to novel talkers based on the statistical properties of their speech as predicted by the ideal adapter framework, we see two potentially fruitful directions. First, we think that perceptual recalibration paradigms should be scaled up to explore the role of natural levels of within-category, within-talker variability and the role of controlled deviation in multiple categories and cue dimensions in recalibration. Second, we think that naturalistic accent adaptation paradigms might be refined to specifically investigate how accent difficulty is driven by deviations-from unaccented speech-in the average value of a cue versus unusual variability in that cue. Given that, across talkers, the average value of some cues varies quite a bit (Newman et al., 2001), while for others it is relatively consistent (Allen, J. L. Miller, \& DeSteno, 2003), it might be expected that listeners will have a harder time adapting to accented speech which is characterized by deviant values of cues that are typically stable across talkers (like VOT Sumner, 2011).

We have discussed how phonetic recalibration is qualitatively predicted by the ideal adapter framework, and presented a model in this framework which captures the incremental build-up of recalibration quantitatively. In the next three sections we show how this framework provides a potentially unifying perspective on phonetic adaptation more broadly. 


\subsubsection{Beyond recalibration: selective adaptation}

Next we apply the ideal adapter framework to a phenomenon known as selective adaptation (Eimas \& Corbit, 1973; Samuel, 1986). Traditionally, selective adaptation is thought to be due to mechanisms that are distinct from those underlying perceptual recalibration. We will show, however, that the cumulative build-up of selective adaptation is captured by the same belief-updating model introduced in the previous sections.

Selective adaptation occurs after repeated exposure to a single phonetic category, and is characterized by a negative aftereffect, where fewer items on a phonetic continuum are classified as the adapted category. For instance, and as we will discuss in more detail below, Vroomen, van Linden, de Gelder, and Bertelson (2007) found that repeated exposure to a prototypical /b/audio-visual adaptor constructed from the $/ \mathrm{b} /$ endpoint of their $/ \mathrm{b} /-/ \mathrm{d} /$ continuum (rather than the ambiguous midpoint) resulted in fewer / b/ responses during test trials (and vice-versa for $/ \mathrm{d} /$ ).

Selective adaptation is broadly considered to be the result of either habituation of "feature detectors" which are sensitive to linguistically-relevant features of the acoustic speech signal, or contrast effects at a categorical level (Samuel, 1986). It is also generally acknowledged that selective adaptation operates at a range of different levels. On the one hand, non-speech phonetic analogues (like isolated F2 and F3 formant transitions) can selectively adapt a place of articulation contrast, which suggests that selective adaptation operates on relatively low-level auditory processing (Samuel \& Kat, 1996). On the other hand, selective adaptation has also been shown to generalize between acoustically different but phonetically similar continua, suggesting that it does not depend solely on acoustic overlap (Samuel \& Kat, 1996; Sawusch, 1977). 


\section{Incremental selective adaptation}
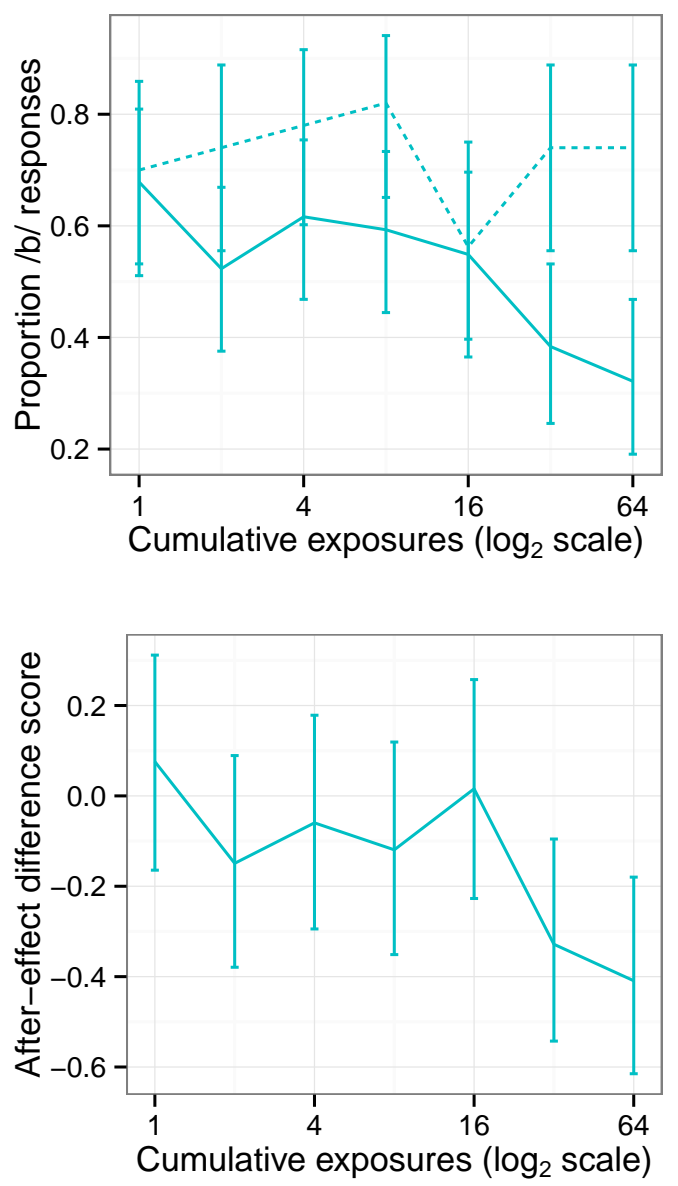

Figure 2.8: Selective adaptation results from Vroomen, van Linden, de Gelder, and Bertelson (2007), showing both the proportion of /b/ responses (top) and the aftereffect difference score (bottom) for the first 64 cumulative exposures in the first exposure block. Error bars indicate $95 \%$ confidence intervals.

As is the case with recalibration, there is little work on how selective adaptation builds up incrementally. Again, one notable exception is Vroomen, van Linden, de Gelder, and Bertelson (2007), who also investigated the build-up of selective adaptation at varying levels of exposure. Selective adaptation was induced by repeated exposure to a prototypical audio-visual adaptor, made using the same video as the ambiguous audio visual stimuli from the recali- 
bration conditions paired with the corresponding category endpoint ( $x=1$ for $/ \mathrm{b} /$ and $x=9$ for $/ \mathrm{d} /$ ). Other than this, the design and procedure was exactly the same as the recalibration condition described above: listeners heard a total of 256 repetitions of one of these adaptors, and were tested on the same audioonly classification test after 1,2, 4, 8, 16, 32, 64, 128, and 256 cumulative adaptor exposures.

Figure 2.8 shows the results for the first 64 cumulative exposures in the first exposure block for selective adaptation from Vroomen, van Linden, de Gelder, and Bertelson (2007). Like with the recalibration conditions, selective adaptation builds up incrementally over the first 64 exposures (although there is quite a bit of noise due to the small number of observations). Can a belief updating model account for this data?
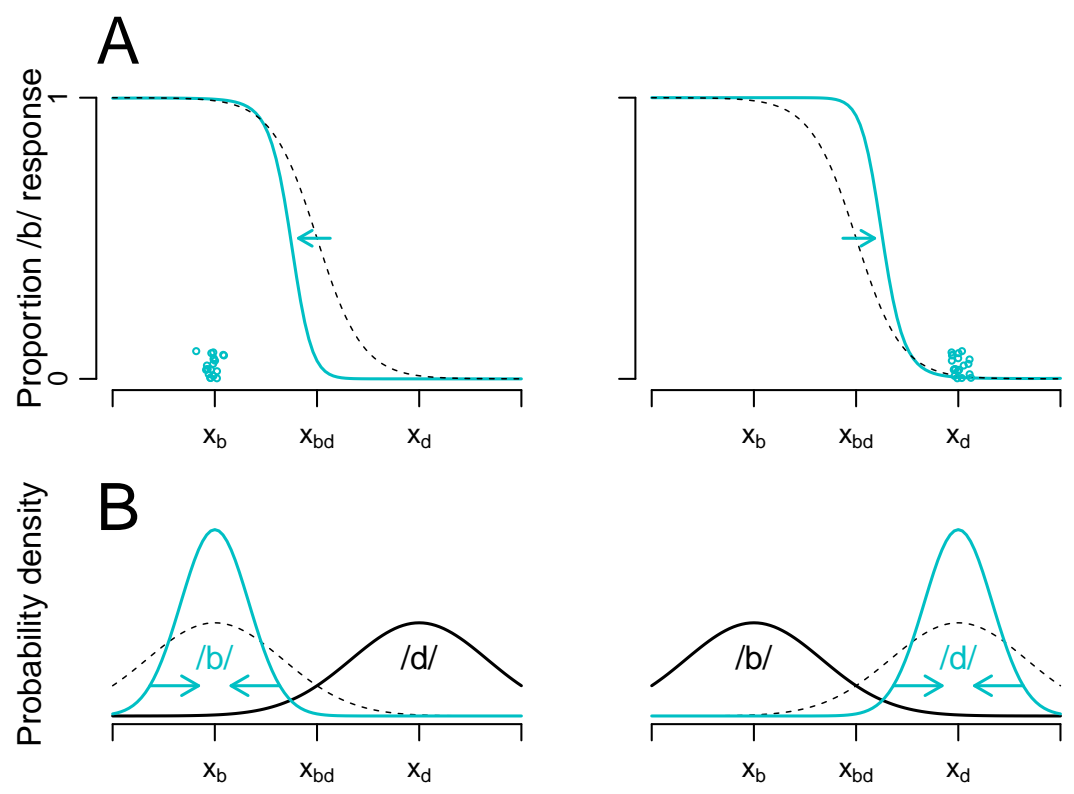

Figure 2.9: Schematic illustration of the results of selective adaptation on classification of a /b/-/d/ continuum (A), and the changes in the listener's beliefs about the underlying distributions which we propose to account for the changes in classification (B). Dashed lines show pre-exposure classification functions and distributions, while solid lines show post-recalibration. Left panels show the results of exposure to prototypical $x_{\mathrm{b}^{\prime}}^{\mathrm{b}}$, and the right to $x_{\mathrm{d}}^{\mathrm{d}}$. 
Qualitatively, the answer is yes, as shown schematically in Figure 2.9. Just like the recalibration condition, the distribution of cues that listeners encounter in the selective adaptation condition is unusual: the exact same cue value is repeated over and over again. ${ }^{15}$ In natural speech there is inevitable variability in the cue values used to realize a single phonetic category, and thus the level of consistency in this experiment is highly unusual. A belief updating model predicts that listeners will adjust their beliefs about the variance of the adapted category as a result of these unusual statistics. The results of adapting to lowvariance exposure to, e.g., a prototypical /b/ is that the /b/ category 'shrinks', and cues values that were previously ambiguous become less likely under /b/ and thus more likely to be classified as /d/ (Figure 2.9, top). This is the negative aftereffect that characterizes selective adaptation.

While few studies have investigated the effect of unusual category variance on subsequent perception, one notable exception is Clayards et al. (2008). They showed that listeners who are exposed to $/ \mathrm{b} /$ and /p/ sounds whose VOTs have low variance show a sharper category boundary than listeners exposed to high variance distributions. Moreover, the difference in category boundary slopes is exactly as predicted by the category variances, via an ideal listener model. This suggests that listeners adjust their categorization behavior based on recently experienced within-category variance of acoustic cues, exactly as the ideal adapter framework predicts.

\section{Modeling the build-up of selective adaptation}

The ideal adapter framework-and the model introduced above-makes further predictions about how classification behavior depends on the amount of

\footnotetext{
${ }^{15}$ There is of course additional perceptual uncertainty or noise variance (Feldman, Griffiths, \& Morgan, 2009), but the combined variance from sensory uncertainty and actual stimulus variance across trials is still less for an identical repeated stimulus than would be expected with normal levels of variability.
} 
exposure, as well as its statistical properties. In order to evaluate the ability of belief updating to quantitatively account for the build up of selective adaptation, we fit the same model that was fit to the recalibration data above to the first 64 exposures of the selective adaptation data from the first block of each participant in Vroomen, van Linden, de Gelder, and Bertelson (2007).

\section{Results}

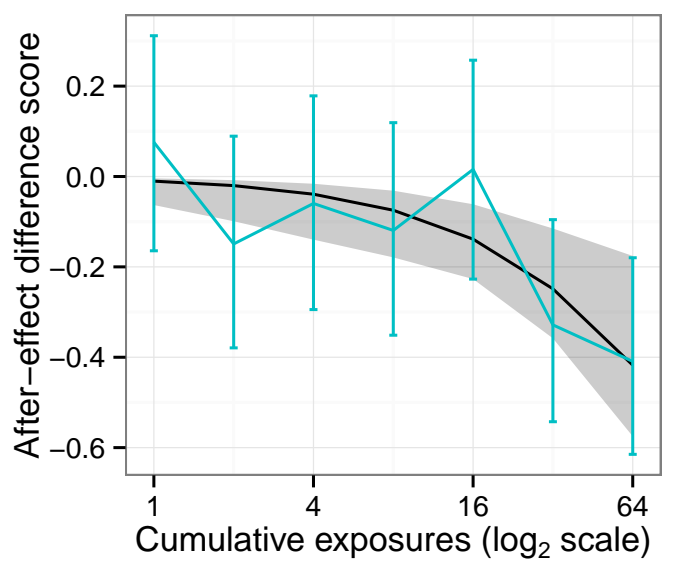

Figure 2.10: Belief-updating model fits to the data from Vroomen, van Linden, de Gelder, and Bertelson (2007) on the build up of selective adaptation after varying levels of cumulative exposure to a prototypical audio-visual adaptor $(x$-axis, on a log scale). The solid black line shows the MAP (maximum a posteriori) estimate predictions $\left(r^{2}=0.83\right)$. The error bars and shaded region show $95 \%$ credible intervals for the data and model predictions, respectively.

The model also fits the selective adaptation data well $\left(r^{2}=0.83\right)$, as shown in Figure 2.10. As the model predicts, selective adaptation starts very weakly, gradually becoming stronger with further exposure. The build-up of selective adaptation also appears to accelerate, unlike recalibration, with further exposure producing even larger increases in the strength of the selective adaptation effect, which is also captured by the model. Thus, while selective adaptation is not usually considered the result of belief updating or distributional learning, 
the same belief updating model which describes well how listeners integrate each observation with their prior beliefs during recalibration also describes the build-up of selective adaptation.

The best-fitting (MAP estimate) hyperparameters for the selective adaptation data are also nearly identical to the recalibration estimates: the effective prior sample size for the variances is $v_{0}=64$ (vs. 71), and for the means, $\kappa_{0}=13$ (vs. 11). The visual cue weight is $w=0.51$ (vs. 0.53). This demonstrates two points. First, as with recalibration, these values correspond to very small effective prior sample sizes, which suggests that listeners do not believe that their vast amount of prior experience with $/ \mathrm{b} / \mathrm{s}$ and $/ \mathrm{d} / \mathrm{s}$ is very relevant in this situation.

Second, even though these parameter values correspond to higher prior confidence in the variance than the mean, the model nevertheless accounts for selective adaptation via shrinking category variance. This is because, unlike the ambiguous adaptor, for the prototypical adaptor there is no difference between the observed and expected category means. In this case, the updated estimate of the variance is simply an average of the prior expected and the observed variance, weighted by the effective prior sample size and actual sample size, respectively (see Appendix A.1.1, Equation (A.7)). For the best-fitting estimate of the effective prior sample size $v_{0}=64$ and an observed variance of zero, this means that the model believes the category variance to have halved after 64 exposures.

\section{Discussion}

These results suggest that, at least for the classification results of Vroomen, van Linden, de Gelder, and Bertelson (2007), it is not necessary to invoke a qualitatively distinct process to explain selective adaptation. The ideal adapter also makes the correct qualitative predictions for a variety of other selective adap- 
tation experiments (e.g., Eimas \& Corbit, 1973; J. L. Miller, Connine, Schermer, \& Kluender, 1983). It might thus be tempting to assume that all selective adaptation effects can be reduced to distributional learning of the type proposed here. Such an account will face several serious challenges. This includes the need to account for complex reaction time effects induced by different types of selective adaptation (Samuel, 1986; Samuel \& Kat, 1996), about which our ideal adapter framework does not yet have anything principled to say. Rather than discuss this and other challenges to an ideal adapter account of selective adaptation here, we merely note that much work remains to be done in fleshing out the predictions of the ideal adapter framework for selective adaptation more generally.

In particular, three directions for future work stand out. First, in order to link the ideal adapter framework to reaction time data, process models have to be developed. Second, our account of selective adaptation depends on the listener's perceived variance of each distribution, which may not be the same as the measured variance of some physical cue. We have made the simplifying assumption here that the perceived variance is the same as the actual variance. For extreme cases, like the case of selective adaptation studied here, where there is no (or minimal) variance in the physical signal, this assumption leads to implausible asymptotic behavior: with more and more exposure, the category should shrink to nothing, leading to an asymptotic aftereffect of -1 . Of course, between 128 and 256 exposures, the negative aftereffect induced by selective adaptation appears to continue to grow stronger (Figure 2.8), suggesting that it has not yet reached its actual asymptote, but we do not know what that is for this paradigm. The distinction between perceived and actual variance becomes even more important when making quantitative predictions about different levels of variance. If the sensory uncertainty (or noisiness in the perceptual system) contributes substantially to the perceived variance (for 
preliminary evidence, see Clayards et al., 2008; Feldman, Griffiths, \& Morgan, 2009; Kronrod, Coppess, \& Feldman, 2012), this is expected to reduce or eliminate predicted effects of variation in physical variance.

Third, our account opens the door to alternative interpretations of particularly challenging aspects of listeners' classification behavior after selective adaptation (Kleinschmidt, Emberson, Doko, Edelman, \& Raizada, 2015). More generally, recent work on non-linguistic sensory adaptation (mostly in lowlevel vision and audition) has revealed that many negative aftereffects which were originally attributed to the fatigue of neuronal feature detectors are better explained by neural populations adjusting their processing to maximize the transmission of information about the current stimulus ensemble (Brenner et al., 2000; Fairhall et al., 2001; Gutfreund, 2012; Kohn, 2007; Sharpee et al., 2006; Webster et al., 2005), which parallels recent developments in the understanding of perceptual learning in low-level perceptual tasks (Bejjanki, Beck, Lu, \& Pouget, 2011; Harris, Gliksberg, \& Sagi, 2012). Maximizing information transmission depends on the statistics of the environment at many different levels.

For speech perception, sometimes the relevant statistics are at the level of the phonetic generative model-the cue distributions for each category-but sometimes they are at a different level, such as the distributions of categories themselves or the spectral characteristics of background noise which must be ignored. For instance, J. Huang and Holt (2012) found that the classification boundary between "bet" and "but" could be manipulated simply by preceding exposure to a pure tone: when the frequency was near the second formant frequency of "bet", listeners made fewer "bet" responses. This seems incompatible with the idea that selective adaptation is due to the listener updating their beliefs about the distribution of cues associated with a particular category, but it is entirely consistent with a more general view that adaptation reflects changes in the listener's beliefs about which cues are more likely to 
occur-or be behaviorally relevant-across different levels of processing. That is, even though selective adaptation may not always represent updating beliefs about the talker's generative model, it may still serve the same purpose: efficient processing of linguistically-relevant acoustic signals in a world where the statistical properties of those signals vary across situations (Kleinschmidt, Emberson, et al., 2015). However, straightforward application of a model like the one presented here to perceptual inference at lower levels has not been successful (Stocker \& Simoncelli, 2006) and more work remains to flesh out this connection.

For the current purpose, it is sufficient to conclude that not everything that looks like selective adaptation requires an explanation in terms of a separate computation. Despite the fact that recalibration and selective adaptation are typically considered to be qualitatively distinct phenomena, we have shown that a single belief updating model can account for the early, incremental buildup of both of these effects. This is achieved under essentially identical assumptions about prior effective sample sizes and the audio-visual cue weight (the hyperparameters in our model). We have also shown that the process of the listener updating their beliefs about category variance provides a likely explanation for at least some adaptive behaviors during speech processing (see also Clayards et al., 2008). In the next section, we explore the consequences of this shrinking category variance in response to a repeated adaptor stimulus for prolonged exposure to a repeated stimulus.

\subsubsection{Effects of prolonged repeated exposure to the same stim- ulus}

The ideal adapter framework predicts that when presented with a single, repeated stimulus, the listener should shrink the variance of the repeated 
category. This leads to predictions about the incremental effects of selective adaptation by a prototypical sound (discussed in the previous section), but it also makes an interesting prediction about repeated exposure to an ambiguous sound. Even though listeners typically do not encounter a physically identical sound repeatedly in real life, the use of repeated sounds is common in perceptual recalibration experiments, which often use a single repeated token (e.g., Bertelson et al., 2003; Vroomen, van Linden, Keetels, et al., 2004) or multiple words where the critical segment is replaced with the same ambiguous sound (e.g., Norris et al., 2003; Samuel, 2001; but see Kraljic \& Samuel, 2005; Reinisch \& Holt, 2014).

Recall that according to the belief-updating model presented above, in the recalibration condition of Vroomen, van Linden, de Gelder, and Bertelson (2007) listeners do not perceive the adaptor cue value as fully ambiguous. Rather, their perceived cue value combines the ambiguous acoustic cue value and the prototypical visual cue value with roughly equal weight (visual cue weight parameter $w=0.53$ ). The belief-updating model predicts that with repeated exposure to this not-quite-ambiguous adaptor, after the shift in the mean to the observed cue value, the low observed variance will eventually lead to the category shrinking and pulling back from the middle of the continuum. This prediction is illustrated in Figure 2.11.

This results in an eventual decrease in the likelihood that the adapted category assigns to the auditorily ambiguous test stimuli in the middle of the continuum and predicts that the positive after-effect associated with recalibration will eventually weaken and possibly even reverse if the category pulls back far enough. We have seen a hint that this may in fact occur: recalibration seems to effectively level off by 64 cumulative exposures in the data from Vroomen, van Linden, de Gelder, and Bertelson (2007). In this section, we further test this prediction by looking at the additional data that Vroomen, van Linden, de 

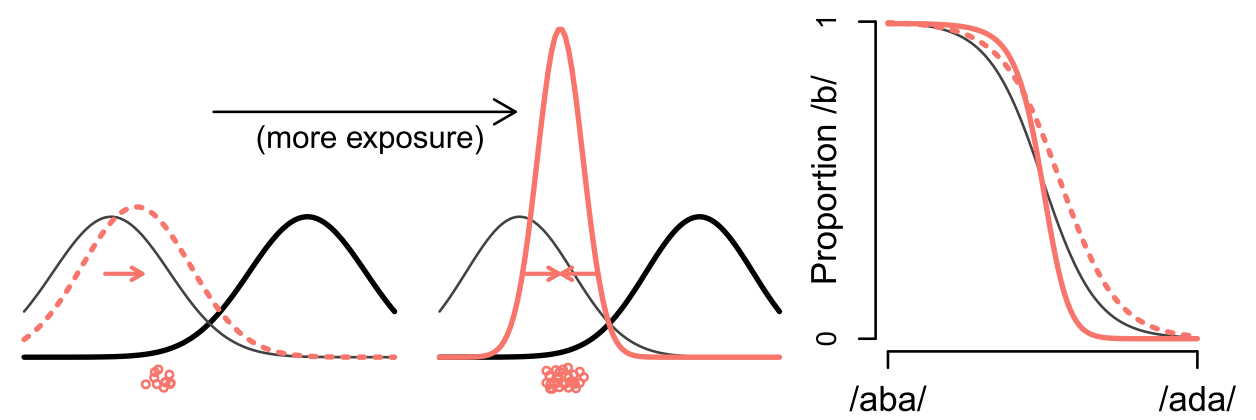

Figure 2.11: Schematic illustration of the predicted trade-off between shifts in mean and change in variance. With exposure to tightly clustered (or repeated) stimuli which are perceived as not fully ambiguous, the ideal adapter predicts that the initial shift in mean should lead to a positive aftereffect with small amounts of exposure (left, and dashed line), while the low variance of the repeated adaptor eventually leads to a neutral or even negative aftereffect with prolonged exposure (middle).

Gelder, and Bertelson (2007) collected for up to 256 cumulative exposures.

\section{Data}

As discussed above, Vroomen, van Linden, de Gelder, and Bertelson (2007) exposed listeners to a total of 256 repetitions of the audiovisual adaptor. Figure 2.12 shows the results from both conditions, including the test trials at 128 and 256 cumulative exposures. As qualitatively predicted, the negative aftereffect associated with selective adaptation grows stronger with further exposure, while the positive aftereffect associated with recalibration plateaus and even begin to decline after 256 exposures. ${ }^{16}$

\footnotetext{
${ }^{16}$ While the decline in recalibration appears to be relatively modest in the figure here, Vroomen, van Linden, de Gelder, and Bertelson (2007) actually found much stronger declines in recalibration in their full data set, where recalibration essentially disappears by 256 exposures. Our replication presented in the next section establishes that such a decline can occur in the first block (Figure 2.15), suggesting that the failure to observe it strongly in the first block of Vroomen, van Linden, de Gelder, and Bertelson (2007) is due to individual differences, which are substantial.
} 


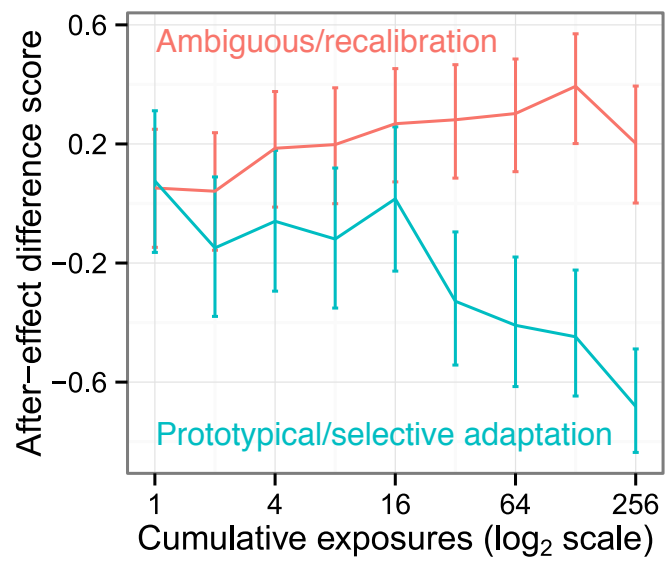

Figure 2.12: Results from Vroomen, van Linden, de Gelder, and Bertelson (2007), showing the full 256 exposures. Red/top curve: ambiguous audiovisual adaptor (recalibration). Blue/bottom curve: prototypical audio-visual adaptor (selective adaptation).

\section{Results and discussion}

To quantify these predictions and test whether the belief updating model can account for the decrease in recalibration, we fit it to the data from all 256 exposures to the ambiguous and prototypical adaptors. The model fits well when fit to either the ambiguous or prototypical conditions individually $\left(r^{2}=0.86\right.$ in both cases). To further test the model's ability to provide a unified explanation of recalibration and selective adaptation, we fit the model to both conditions simultaneously.

Figure 2.13 shows that the model simultaneously fits the behavioral data in both conditions quite well $\left(r^{2}=0.93\right.$ overall, and $r^{2}=0.86$ and 0.85 for the ambiguous and prototypical subsets, respectively). There is no loss of goodnessof-fit from fitting both conditions simultaneously. The best fitting hyperparameters were $v_{0}=100, \kappa_{0}=17$, and $w=0.47$. These hyperparameters are very similar to the best-fitting hyperparameters for the first 64 exposures only, as well as the estimates when each condition is fit separately. 


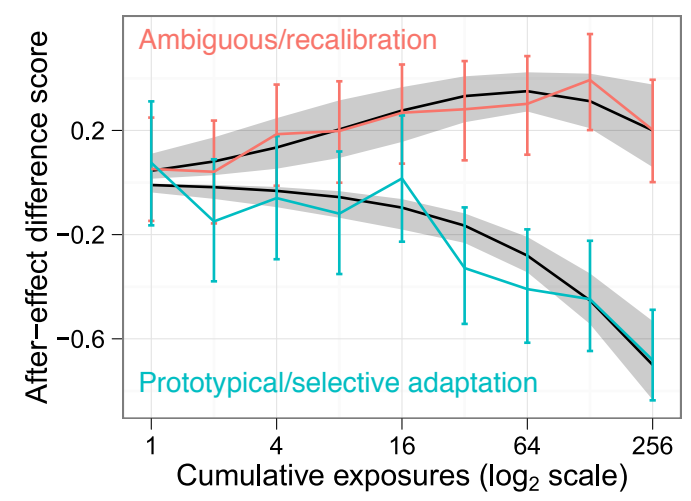

Figure 2.13: Results from fitting the belief updating model to all 256 exposures in both conditions from Vroomen, van Linden, de Gelder, and Bertelson (2007) simultaneously. Model predictions correspond to MAP-estimate hyperparameters of $v_{0}=100, \kappa_{0}=17$, and $w=0.47$.

In particular, the best fitting models for the initial build-up (first 64 exposures) and prolonged exposure (256 exposures) place roughly the same weight on the visual cue $(w=0.53$ vs. $w=0.47$, respectively, where $w$ could range from 0 to 1 ), even though the decay in recalibration is virtually absent during the first 64 exposures. That is, just based on the rate at which recalibration initially accumulates, the model predicts the later decay without further assumptions.

\subsubsection{Exploring the predictive power of the ideal adapter}

One of the benefits of a model like we present here is that it makes quantitative predictions which go beyond existing data. In this section, we explore the ideal adapter framework's predictive power. The fact that the ambiguous and prototypical conditions from Vroomen, van Linden, de Gelder, and Bertelson (2007) can be accounted for by a single set of belief updating model hyperparameters suggests that the recalibration and selective adaptation effects in this experiment are not qualitatively distinct but rather endpoints on a continuum 
of adaptation effects. The ideal adapter framework predicts that for observed cues which are less ambiguous, the low variance of the adaptor distribution will be detectable with fewer observations, causing an earlier (and lower) peak in the positive aftereffect detected in the recalibration condition.

In order to test this prediction, we replicate and extend the design of Vroomen, van Linden, de Gelder, and Bertelson (2007), adding intermediate conditions where the acoustic component of the audio-visual adaptor is neither fully ambiguous nor fully prototypical, but somewhere in between. As a strong test of the predictive power of the model, we ask whether hyperparameters fit to the ambiguous/recalibration and prototypical/selective adaptation conditions in our replication can be used to predict adaptation behavior in new situations which have not been studied before.

\section{Methods}

We developed a novel web-based paradigm to efficiently collect phonetic categorization data from a large number of participants, adhering as closely as possible to the methods of Vroomen, van Linden, de Gelder, and Bertelson (2007). In addition to the ambiguous and prototypical conditions of Vroomen, van Linden, de Gelder, and Bertelson (2007), we added two intermediate conditions: intermediate-ambiguous, and intermediate-prototypical.

Stimuli Stimuli were identical to those used by Vroomen, van Linden, de Gelder, and Bertelson (2007), who generously shared their materials. The audio stimuli were items from a nine-item synthetic /aba/-/ada/ continuum, created by shifting F2 locus in equal mel steps from a prototypical /aba/ value to a prototypical /ada/ value, holding other parameters constant (Vroomen, van Linden, Keetels, et al., 2004). The visual stimuli were natural videos of a male talker articulating /aba/ and /ada/. 
Audio-visual adaptors for the ambiguous and prototypical conditions were constructed as in Vroomen, van Linden, de Gelder, and Bertelson (2007), by matching the video with the participant's most ambiguous continuum item $x_{\mathrm{bd}}$ or the corresponding continuum endpoint, respectively. For the two intermediate conditions, the audio component was offset by one (intermediateambiguous) or two (intermediate-prototypical) positions towards the video category endpoint (Figure 2.14).

Exposure stimuli by condition (visual /b/)

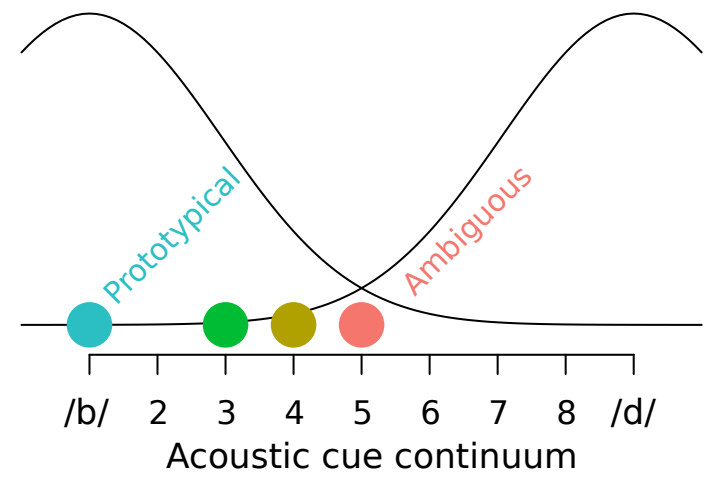

Figure 2.14: Construction of stimuli for four conditions with visual /b/ (visual /d/ is analogously the mirror image).

Procedure Participants first performed the same pre-test /b/-/d/ classification task as in Vroomen, van Linden, de Gelder, and Bertelson (2007) (described above). After the calibration phase, participants were split into four exposure conditions (Figure 2.14): ambiguous and prototypical conditions as in Vroomen, van Linden, de Gelder, and Bertelson (2007), plus two intermediate conditions. For the intermediate-ambiguous condition, the adaptors were constructed from the continuum item one position over from the most ambiguous position, in the direction of the endpoint corresponding to the video category. The intermediate-prototypical adaptors were constructed from the continuum item two positions over from the most ambiguous item. Each participant did 
two exposure blocks, one /b/ and one /d/.

Each block consisted of a total of 128 audio-visual exposure trials. Audioonly $/ \mathrm{b} /-/ \mathrm{d} /$ test trials were interspersed after $1,2,4,8,16,32,64,96$, and 128 cumulative exposures, in blocks of 6 or 12 trials ( 2 or 4 repetitions of each participant's 3 most ambiguous stimuli). Following the first exposure block, participants took a short break and completed the second block, with an audiovisual exposure stimulus from the opposite category but same condition.

The experiment was conducted over the web, via Amazon's Mechanical Turk crowd-sourcing service using a custom Javascript application. ${ }^{17}$ Response keys and block order were counter-balanced across participants. The experiment took no longer than 45 minutes to complete.

Participants A total of 280 participants were recruited. Since participants were being run remotely, using a variety of audio equipment to complete the experiment, a number of quality checks were required. First, our task was only made available to workers whose location was listed as the US and had more than $95 \%$ of their previous work accepted for payment (an automatic quality control measure offered by Amazon). Second, based on the calibration task, participants with unusual category boundaries (most ambiguous stimulus not one of the middle three positions 4, 5, or 6 found by Vroomen, van Linden, de Gelder, \& Bertelson, 2007) were automatically excluded from the remainder of the experiment. 60 participants were excluded for this reason. Third, participants who classified the two endpoint stimuli and their nearest neighbors ( $x=1,2,8$, or 9 ) with less than $70 \%$ accuracy were also excluded. 25 additional participants were excluded for this reason.

Fourth, in order to ensure that participants were actually watching the

\footnotetext{
${ }^{17}$ This source code and a working demo are available from http://hlplab.wordpress.com/ 2013/09/22/phonetics-online/
} 
videos during the exposure phase, catch trials were interspersed throughout exposure (as in Vroomen, van Linden, de Gelder, \& Bertelson, 2007). These trials were identical to normal audio-visual exposure trials, except for a small white dot which flashed for one frame above the talker's lip. On these trials, participants were instructed to press the space bar to indicate they saw the dot. Participants who missed more than a total of $20 \%$ or more than $50 \%$ on any one block were excluded from analysis and replaced (13 participants). After this exclusion, the overall catch trial accuracy rate was 96\% (compared to 93\% reported by Vroomen, van Linden, de Gelder, \& Bertelson, 2007).

Following good statistical procedure (Simmons, Nelson, \& Simonsohn, 2011), these exclusion criteria were fixed before beginning data collection and automatically executed by our experiment software. Data from a total of 182 participants remained for analysis.

Modeling We fit the belief updating model introduced above against only the ambiguous and prototypical conditions. We chose to fit these conditions for two reasons. First, we want to replicate the model fits to the data from Vroomen, van Linden, de Gelder, and Bertelson (2007) on a novel language (and in our novel paradigm). Second, we want to test the ability of the model predict the effect of cumulative exposure in the two novel, intermediate conditions, based on conditions which have already been investigated. The posterior distribution of model hyperparameters $\left(v_{0}, \kappa_{0}\right.$, and $\left.w\right)$ given the ambiguous and prototypical conditions can be used to generate predictions for the intermediate conditions (by plugging in the adaptor values, offsets of one and two from the most ambiguous stimulus, $x_{\mathrm{bd}} \pm 1$ and $x_{\mathrm{bd}} \pm 2$, for the intermediate-ambiguous and intermediate-prototypical conditions, respectively, with the sign determined by the visual component). 


\section{Results}

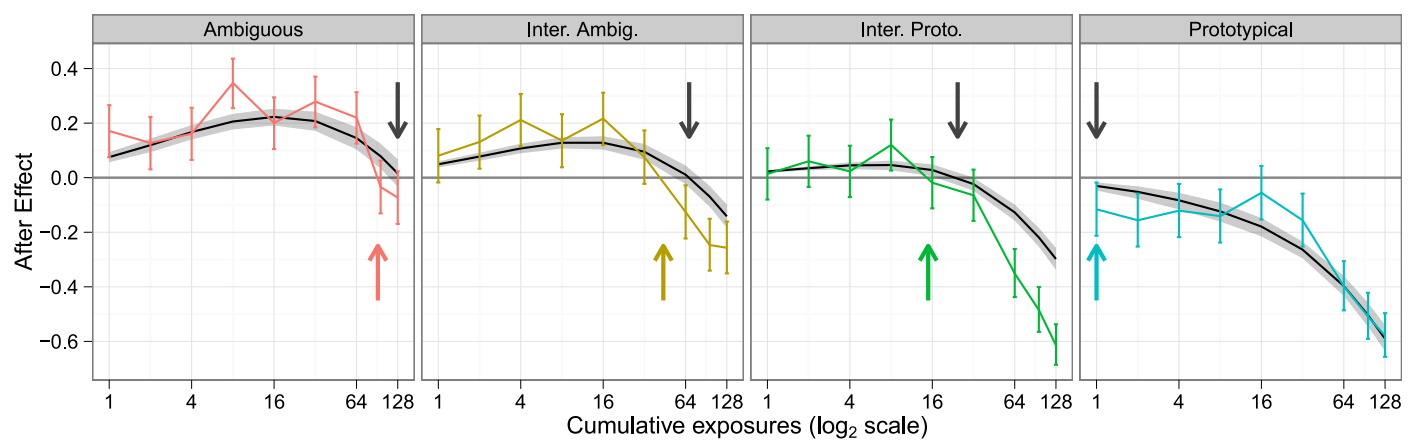

Figure 2.15: Results from all four conditions in the first exposure block: ambiguous, intermediate-ambiguous, intermediate-prototypical, and prototypical (colored lines with error bars showing 95\% confidence intervals). Model fits (black lines with 95\% confidence interval ribbons) are generated based only on the ambiguous and prototypical conditions $\left(r^{2}=0.91\right)$; for the two intermediate conditions the model makes the predictions shown $\left(r^{2}=0.96\right)$. Colored arrows show the amount of exposure required for behavior to switch from recalibration-like (positive after-effect) to selective adaptation-like (negative after-effect), which decreases as the adaptor stimulus goes from ambiguous to prototypical, as predicted by the model (black arrows; see Figure 2.16).

Here we focus on the evaluation of the incremental belief updating model developed in the previous sections. Additional data analyses confirmed that our web-based design replicates the results of Vroomen, van Linden, de Gelder, and Bertelson (2007) and further supports our interpretation of the data. These analyses can be found in the Supplementary Material to this article. ${ }^{18}$ Figure 2.15 shows the results from the four conditions, along with the predictions from the model fit to the ambiguous and prototypical conditions. First, our results replicate those of Vroomen, van Linden, de Gelder, and Bertelson (2007). Exposure to the prototypical adaptor leads to negative aftereffects which build up gradually and become stronger throughout exposure, while the exposure to the ambiguous adaptor leads to positive aftereffects which peak and eventu-

\footnotetext{
${ }^{18}$ The Supplementary Material are available from http://www.bcs.rochester.edu/people/ dkleinschmidt/pubs/KleinschmidtJaeger-SupplementaryMaterial.pdf
} 
ally fade, becoming negative after 128 exposures (see Supplementary Material). In fact, the recalibration decline that we find is even stronger than that found by Vroomen, van Linden, de Gelder, and Bertelson (2007), with 128 exposures being enough to almost completely erase any positive aftereffect. Vroomen, van Linden, de Gelder, and Bertelson (2007) observed such a reversal after 256 exposures in their full data set, but only a slight dip in the strength of the recalibration effect by the end of the first block of exposure (Figure 2.12). Our belief updating model fits the data from the ambiguous and prototypical conditions quite well ( $r^{2}=0.91$ overall, vs. $r^{2}=0.93$ when fit to Vroomen, van Linden, de Gelder, \& Bertelson, 2007).

Second, these results validate the predictions of the belief updating model for intermediate conditions, both qualitatively and quantitatively. Qualitatively, as predicted, the two intermediate conditions reveal that the ambiguous and prototypical conditions are endpoints of a continuum of adaptation effects. This is visually clear from Figure 2.15 and also borne out by statistical analysis (see Supplementary Material). Quantitatively, the belief updating model hyperparameters that were fit only to the ambiguous and prototypical conditions accurately predict the adaptation build-up in the two intermediate conditions. In fact, the fit to the intermediate conditions is as good as the fit to the conditions the model was trained on $\left(r^{2}=0.96\right.$ for the two intermediate conditions vs. $r^{2}=0.91$ for the ambiguous and prototypical). This is encouraging given that individual participants show substantial variability in their adaptation behavior and each condition consisted of an entirely different group of participants.

We can further compare the model predictions and the data by looking at the point at which the aftereffect crosses over from being positive (recalibrationlike) to negative (selective-adaptation-like). In Figure 2.15, these cross-over points are indicated by arrows-black for the model predictions and colored 


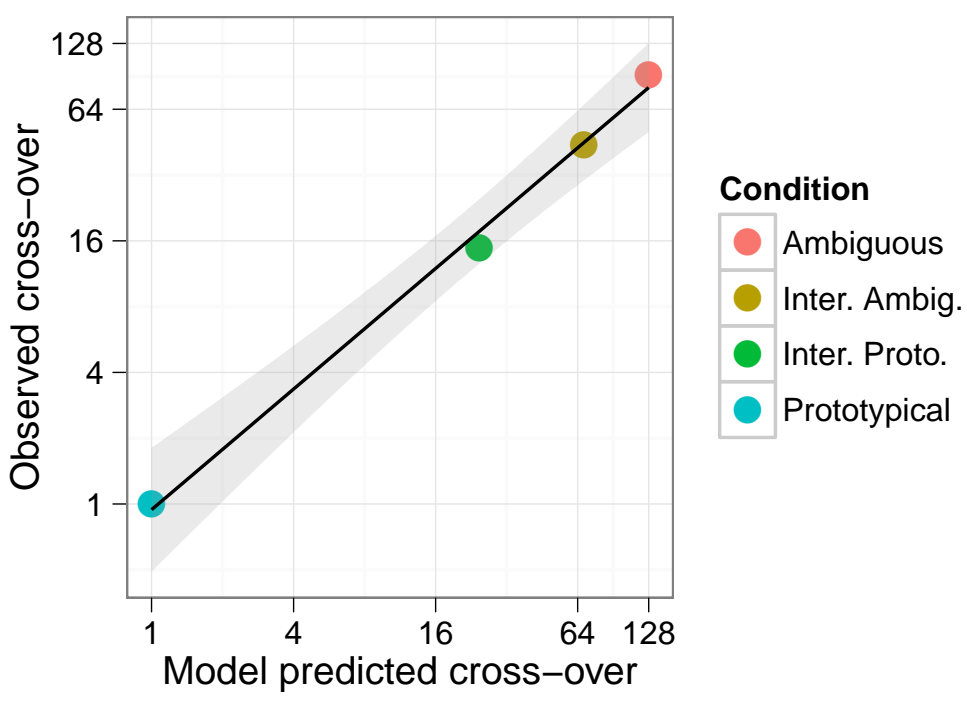

Figure 2.16: The belief-updating model predicts when behavior should switch from recalibration-like to selective adaptation-like (see Figure 2.15). Each dot shows the model predicted cross-over point ( $x$-axis) versus the actual observed cross-over point ( $y$-axis), with a linear regression fit to these four points showing close agreement.

for the data-and Figure 2.16 shows that the observed and model-predicted cross-over points are strongly correlated, even though the cross-over point itself was not explicitly fit to the data. Thus, the belief updating model very effectively predicts the point at which behavior switches from recalibration-like to selective-adaptation-like for intermediate adaptors of varying ambiguity, based solely on the fit to ambiguous and prototypical adaptor data.

The best-fitting (MAP estimate) effective prior sample sizes for the category variances and means were small: $v_{0}=88$ and $\kappa_{0}=2.9$, respectively. This again closely resembles what we found for the Vroomen, van Linden, de Gelder, and Bertelson (2007) data. It also corroborates the conclusion that participants in these studies consider their previous experiences with /b/ and /d/ to be not particularly relevant in these situations, and moreover replicates the finding that, for these stimuli and this experimental paradigm, listeners seem 
to place more confidence in their prior beliefs about the category variances than the means ( $v_{0}>\kappa_{0}$ both here and in all previous model fits). Finally, as with the data from Vroomen, van Linden, de Gelder, and Bertelson (2007), the best-fitting cue combination weight had audio and visual cues weighted roughly equally, placing in this case slightly more weight on the visual component $(w=0.63) .{ }^{19}$

\section{Discussion}

These results demonstrate two points. First, by replicating the results of Vroomen, van Linden, de Gelder, and Bertelson (2007), they show that webbased platforms are a viable way to investigate phonetic effects such as recalibration and selective adaptation which depend on the particular acoustic parameters of the stimuli. Most participants were enthusiastic and engaged in the study, and using sensible exclusion (based on pre-test performance or catch trials during exposure) those who are not fully attentive or who cannot hear the stimuli properly can be excluded.

Second, the ideal adapter framework for understanding phonetic adaptation presented above can explain both the phonetic recalibration and selective adaptation data from Vroomen, van Linden, de Gelder, and Bertelson (2007) based on the mean (ambiguous vs. prototypical) and variance (none) of the adaptor distributions. This framework predicts that intermediate adaptors should produce intermediate adaptation effects, and moreover, the formal, quantitative model based on these principles makes specific, quantitative predictions about the effect of cumulative exposure to these intermediate adaptors. Both these qualitative and quantitative predictions were borne out in the data presented here.

\footnotetext{
${ }^{19}$ The slightly higher weight for visual cues in our data might be a consequence of using stimuli derived from a Dutch /b/-/d/ continuum, which are similar but presumably not identical to those typically experienced in American English.
} 


\subsubsection{Concluding Part I: Adaptation as inference under uncer- tainty about the statistics of the generative model}

In this first part of this article, we have formulated the ideal adaptor framework, building on previous work on speech perception within the ideal listener framework (Clayards et al., 2008; Feldman, Griffiths, \& Morgan, 2009; Norris \& McQueen, 2008; Sonderegger \& Yu, 2010). We have applied the ideal adaptor framework to two phonetic adaptation phenomena commonly considered to be due to distinct mechanisms, perceptual recalibration and selective adaptation. To do so, we derived a Bayesian belief updating model from the ideal adaptor. This framework allowed us to formalize the intuition that perceptual recalibration is a form of distributional (statistical) learning that changes the underlying representations of phonetic categories (e.g., Norris et al., 2003; Kraljic \& Samuel, 2005; Maye, Aslin, \& Tanenhaus, 2008; Bertelson et al., 2003). The Bayesian belief updating model provides a good quantitative fit to perceptual recalibration data $\left(r^{2} \mathrm{~s}>.8\right)$. The model also qualitatively captures the cumulative effect of exposure in perceptual recalibration experiments, including the previously observed reversal of initial effects after prolonged exposure to the same stimulus (Vroomen, van Linden, de Gelder, \& Bertelson, 2007).

Going beyond perceptual recalibration, the model suggests selective adaptation, too, is at least in part due to distributional learning similar or identical to that observed during perceptual recalibration. In addition to good quantitative and qualitative fits to selective adaptation data, our Bayesian belief updating model predicted a continuum from selective adaptation to perceptual recalibration, which was indeed observed. This serves as a demonstration of the ability of the ideal adapter framework to make quantitative predictions that go beyond existing data.

It is worth mentioning that the model which is used to generate those predic- 
tions in this case makes many assumptions that are not intrinsic to the framework but rather are made for convenience, tractability, or to better illustrate the basic mechanics of belief updating (for discussion, see the Appendix). These assumptions are largely justified for this particular experimental paradigm (see Table A.1 in the appendix). They must, however, be considered carefully when thinking about how this particular model might generalize to other paradigms. Support for the ideal adaptor framework as a general framework of adaptation beyond the type of experimental paradigms considered here comes from research finding qualitatively similar patterns of speech adaptation in situations that more closely resemble the complexity of every day speech perception (Eisner \& McQueen, 2006; Reinisch \& Holt, 2014).

\section{Novelty of the ideal adapter: Putting learning front and center}

Many of the ideas we have discussed so far are anticipated in more or less explicit form in previous work on speech perception. Our approach to speech perception is novel in two ways. First, the ideal adapter puts learning front and center in speech perception, as an unavoidable consequence of the combination of the task of the speech perception system-mapping cues to categoriesand the world where that task is carried out-where the cue-category mapping varies from one situation to the next. In this way of looking at the speech perception system, learning or adaptation is a necessary part of normal speech perception. Existing models of speech perception-Bayesian and otherwisefor the most part do not learn or adapt (Clayards et al., 2008; Feldman, Griffiths, \& Morgan, 2009; McClelland \& Elman, 1986; Norris, 1994; Norris, McQueen, \& Cutler, 2000). This is, in general, not an in-principle limitation of these modeling frameworks, but rather reflects simplifying assumptions made for the sake of tractability in previous work. 
There are only a few models of speech perception that learn, ${ }^{20}$ all of which have been proposed in recent years based on findings that speech perception is highly flexible (Lancia \& Winter, 2013; Mirman, McClelland, \& Holt, 2006). These models are connectionist models that adjust feedforward weights from acoustic cues to phonetic categories using Hebbian mechanisms. We postpone further discussion of these models until Part III below, except to say that they have only been applied qualitatively to the asymptotic effects of phonetic recalibration (if they have been directly applied to phonetic recalibration at all). As such, it is not clear whether they can, like the belief updating model we present here, simultaneously account for selective adaptation as well, or the detailed effects of cumulative exposure on the size and direction of phonetic adaptation. Another class of models that are broadly compatible with the ideal adapter is episodic or exemplar models (e.g., Johnson, 1997a; Goldinger, 1998; Pierrehumbert, 2003), which learn the distribution of sounds corresponding to linguistic units implicitly by storing raw acoustic traces. However, as we will discuss at length in Part II, despite similar motivations, the ideal adapter framework differs in an important way, because it abstracts away from individual episodes, both at the level of phonetic categories (or some other sublexical level of representation) and at the level of individual talkers and groups of talkers.

A deeper problem with existing models - even those that learn - is that they don't address how listeners manage to balance stability and plasticity in speech perception. Rather, learning rate is treated as a free parameter which is tuned such that the model behaves in a reasonable way. A model of the speech perception system ultimately has to address how stability and plasticity are balanced, because there is a wealth of evidence that people manage to do this very well. While the belief updating model we have presented above has free parameters that control the learning rate (the prior confidence parameters) and

\footnotetext{
${ }^{20}$ Leaving aside for the moment models of acquisition, which we will discuss below.
} 
which were fit to the data, the ideal adapter framework provides a more principled way to approach the stability-plasticity trade-off, which we turn to in the second, more speculative and forward-looking part of this paper.

\subsection{Part II}

Human speech perception is characterized by both extreme flexibility and stability. Listeners can rapidly adapt to a novel pronunciation while not losing the ability to efficiently comprehend standard pronunciations. Part II of this paper focuses on the balance between stability and flexibility, and in particular howin the ideal adapter framework - it is related to the structure of the world that the speech perception system has to operate in. There are two main aspects to this structure.

First, the generative model-the statistical properties of speech sounds for each phonetic category - can be different from one situation to the next. If the listener's beliefs about these statistics are substantially different from the current situation's actual generative model, then comprehension can be slowed or even incorrect. Situation-by-situation differences in the generative model require that listeners continuously infer the appropriate generative model, combining their prior expectations with current observations. In Part I we illustrated how phonetic adaptation can be understood as incremental belief updating, focusing intentionally on novel situations where listeners' prior experience was not likely to be very informative, allowing the influence of each additional observation to be seen more clearly.

However, there is a second relevant aspect to the speech perception system: generative models do not vary arbitrarily across situations, but are rather tied to, for example, who is talking. This means that listeners can expect to encounter the same-or similar-generative models again and again (and some more 
than others). In Part II of this paper, we focus on these similarities across situations that a listener encounters in the world, and discuss how the ideal adapter framework links this structure-with both predictable and unpredictable variation in generative models-with the stability and flexibility of the speech perception system. While a lot of future work is required to flesh out a computational cognitive model, we will present a tentative outline of how the ideal adapter framework formalizes the link between the distribution of generative models in the world, listeners' prior expectations, and their behavior. The ideal adapter framework's basic predictions are qualitatively supported by the available behavioral evidence. At the same time, the ideal adapter framework identifies a number of questions that we consider particularly critical for future research.

\section{Recognize the familiar}

While we have assumed that adaptation to a novel talker does not start from scratch, but rather from some form of prior beliefs about phonetic distributions (literally, the prior in the Bayesian belief-updating model introduced in Part I), we have so far paid little attention to the properties of this prior knowledge. For the examples entertained in Part I, such as the speech a listener is exposed to in a perceptual learning experiment, the specific prior beliefs that the listener brings to the situation are of little consequence because they will quickly be overwhelmed by the input listeners receive from the novel talker. In everyday life, however, listeners will not only encounter talkers that differ starkly from previously experienced talkers. Instead, some talkers will have been encountered before and others will resemble previously experienced talkers to varying degrees. This means that different aspects of previous experience bear more or less strongly on the current situation. How listeners determine which previous experiences are the most relevant in a given situation is one of the goals of the 
second part of this article.

Consider, for example, what happens when you walk into a room where your best friend is talking. You can recognize them based on a variety of things, perhaps including their face, distinctive clothes they happen to be wearing, the fact that they are standing in a room in their own house, or the timbre of their voice. Because every individual speaks slightly differently, your best friend sounds different from other talkers, and it would be beneficial to be able to just "swap in" their particular cue statistics based on your vast experience with their speech in the past. Anecdotally, this is what happens when we encounter a highly familiar talker, and indeed it is broadly accepted and empirically supported that we have such talker-specific representations, and use them online in speech perception (Creel, Aslin, \& Tanenhaus, 2008; Creel \& Bregman, 2011; Goldinger, 1996; Nygaard \& Pisoni, 1998; Palmeri, Goldinger, \& Pisoni, 1993; Remez, Fellowes, \& Rubin, 1997), although questions remain about the limits of this ability (James S Magnuson \& Nusbaum, 2007; Pardo \& Remez, 2006).

Talker-specificity in speech perception reveals an important insight: knowledge about the statistics of speech cues needs to be structured to provide the full benefit to the listener. This has long been recognized by, for example, exemplar-based theories of speech perception, where storage of rich acoustic details of each episode leads naturally to persistent, talker-specific representations of the variability of speech sounds, either implicitly or explicitly (Goldinger, 1996, 1998; Johnson, 1997b, 2006; Pierrehumbert, 2003). In the language of the ideal adapter framework, the presence of talker-specificity means that, far from simply being engaged in continuous statistical learning, listeners are in fact using their previous experience to at the very least determine where to start such statistical learning in each situation. It is this second type of inference-inferences about what aspect of previous experience are most relevant to the current situation-that prevents listeners from having to re-adapt 
from scratch every time they encounter a talker (whether it is a novel talker or a previously encountered one). Although the existence of talker-specific knowledge is now broadly accepted, the consequences that the existence of such knowledge has for understanding speech perception is perhaps still underappreciated. Specifically, most previously proposed models of speech perception, with the exception of certain exemplar-based approaches (Goldinger, 1998; Johnson, 1997b; Pierrehumbert, 2003), either do not learn (Clayards et al., 2008; Feldman, Griffiths, \& Morgan, 2009; Norris \& McQueen, 2008) or are what we will call 'flat' learners, without the ability to induce structure over talkers (Feldman, Griffiths, Goldwater, \& Morgan, 2013; Lancia \& Winter, 2013; McMurray, Aslin, \& Toscano, 2009; Mirman et al., 2006; Vallabha et al., 2007). These models are insufficient to account for talker-specificity and related phenomena discussed below. Even looking beyond speech perception into the burgeoning literature on learning in the face of (latently) non-stationary statistics (e.g., Cho et al., 2002; Gallistel et al., 2001), most existing models cannot account for some of the basic properties of speech perception discussed here. ${ }^{21}$ We return to this point below, as it motivates the proposal we lay out in this second part of the article.

\section{Generalize to the similar}

There is also evidence that the structure of listeners' previous knowledge extends beyond the level of particular individual talkers. To take a somewhat extreme example, one of our colleagues moved from New York to Northern England, where a very different dialect of English is spoken. Initially, he had a great deal of difficulty understanding what anyone was saying, but after some number of months he found that it became easier to comprehend this

\footnotetext{
${ }^{21}$ This also makes speech perception a potentially productive test domain for further development of general models of learning in a non-stationary world.
} 
accented speech, even when it was spoken by particular individuals he had never met before (Farmer, personal communication). In the language of the ideal adapter, we might say that our colleague learned something about how the cue statistics-or generative models-vary across individual talkers who share this accent, and that this gave him a head start in adapting to a new, but similarlyaccented talker. Although somewhat less well-studied than talker-specificity, there is some evidence that people are broadly capable of such generalization, using their experience with groups of talkers to guide speech perception (Bradlow \& Bent, 2008; Baese-berk et al., 2013; Creel \& Bregman, 2011; Johnson et al., 1999; Johnson, 2006; Niedzielski, 1999; Sidaras, Alexander, \& Nygaard, 2009). There are, in the world, a range of structures that group talkers, from common language community (leading to dialect and accent groups) to factors like gender or sexual orientation (B. Munson, 2007). The ideal adapter predicts that listeners should pick up on, and take advantage of, these groupings, to the extent that they are informative about the generative models of the corresponding talkers.

In order to take advantage of the structure of how generative models vary in the world, listeners need to learn this structure through experience. Listeners do not directly know how generative models are distributed in the world, just like they do not have direct access to the generative model behind each utterance they observe. This makes the problem of how to take advantage of any structure that might be there in the world another problem of inference under uncertainty. It is clear that listeners need to learn in order to group talkers together, since meeting two talkers with similar generative models could just be a coincidence, but meeting twenty such talkers likely indicates some underlying dialect group. However, this kind of higher-level learning is also required for talker-specificity. That is, in order to benefit from experience with a new talker in future occasions, listeners cannot just adapt to that talker's generative model 
for the current situation, but rather must, after sufficient exposure, remember what they have inferred about that particular talker's speech statistics so that they can deploy that knowledge on future encounters. The need for listeners to induce structure over their previous experiences with different generative models suggests that despite being rapid, adaptation-like that discussed in Part I-should persist because it reflects the listener's attempt to build a model of the current situation which can be useful in the future. This is another way of saying that phonetic adaptation is not just priming but a form of learning about the situation and/or talker (Eisner \& McQueen, 2006; Kraljic \& Samuel, 2005; Kraljic, Samuel, \& Brennan, 2008, among others).

\section{Outline for Part II}

We will expand on these intuitions about how listeners can benefit from picking up on the structure of their experience with different cue statistics, and review the relevant literature. Our first goal is to argue that the ideal adapter framework provides a unifying view that ties together a range of previously stated intuitions and proposals about talker-specificity, generalization, and adaptation in speech perception. Our second goal is to lay out a preliminary formalization of the ideal adapter framework as it relates to how a listener could take advantage of structure in their prior experience. While we will not present any implemented, quantitative model simulations like in Part I, we believe that formalizing the framework has a number of advantages. First, it will highlight the computational parallelism between recognizing familiar talkers, generalizing to similar talkers, and what we've called adaptation to novel talkers in Part I, all of which reflect listeners' attempts to infer the appropriate generative model for the current situation. Second, having a formalization of a theory can sometimes help when our theoretical intuitions break down, such as when, for instance, speech perception is not talker-specific (e.g. Kraljic \& Samuel, 2007). 
Third, formalizing can help guide future research. As will become clear below, the ideal adapter framework puts a major emphasis on statistics of the speech signal, and how those statistics differ across talkers and groups of talkers in the world. Relatively little research has looked at this, and the ideal adapter framework provides a potentially productive link between this kind of data and human behavior.

Part II is organized into four main sections, each of which addresses a different aspect of how the listener's need to infer the appropriate generative model in each situation results in different strategies based on the information available to them from structure in the world (via prior experience) and the speech they observe in that situation. First, we focus on how listeners can benefit from familiarity with a particular talker. Maintaining and using talker-specific beliefs about generative models allows listeners to forgo adaptation altogether when they encounter a familiar talker again. Second, we ask how listeners get to the point of having such talker-specific beliefs. Every familiar talker was once novel, and became familiar through experience. Combining the beliefupdating logic that was the focus of Part I with talker-specific beliefs leads to the ideal adapter's prediction of talker-specific adaptation. Third, we turn to the question of where a listener starts when adapting to an unfamiliar talker. Even if a particular individual is unfamiliar, the listener often has experience with other similar talkers that can be informative. We will discuss evidence that listeners are indeed sensitive to this information, and how it can be formalized in the ideal adapter framework. Fourth, and finally, we tie all three strategies together, and discuss how connections between adaptation, recognition, and generalization are demonstrated by how listeners behave when they are not entirely sure what prior experience is more relevant. 


\subsubsection{Recognizing the familiar: Talker-specificity}

We begin with situations in which stable beliefs about the generative model are maximally beneficial: particular familiar talkers. Listeners can benefit from experience with a familiar talker to the extent that they consistently use a particular generative model-producing certain cue distributions-that is different from other talkers. By maintaining stable representations of that talker's particular generative model, the listener would be able to deploy those representations when the talker is encountered again in the future, removing the need to adapt to them again. If, as the ideal adapter predicts, listeners are taking advantage of this structure-that talkers tend to use the same generative model consistently-then listeners should generally process speech in a talkerspecific way, and specifically should process speech from familiar talkers faster, more accurately, and more robustly.

\section{What do we know about talker-specificity?}

Talker-specificity is one of the best-studied and uncontroversial features of human speech perception. Talker-specificity is observed both in the form of the ability of listeners to explicitly identify particular individual talkers based on their speech, even when it is highly degraded (Bricker \& Pruzansky, 1966; Palmeri et al., 1993; Remez, Fellowes, \& Rubin, 1997; Sheffert, Pisoni, Fellowes, \& Remez, 2002). Offline measures of speech processing also show talkerspecificity. For instance, words are better remembered when they're spoken by the same talker at study and test (Goldinger, 1996; Palmeri et al., 1993). However listeners also use talker-specific information to benefit processing. Listeners are faster and more accurate at comprehending speech in noise when it was produced by familiar talkers, both for unaccented (Nygaard \& Pisoni, 1998) and accented (Clarke-Davidson \& Garrett, 2004) talkers. These effects 
seem to operate at a low level in speech perception, with online measures like eye-tracking suggesting that listeners use experience with talker-specific productions as early as it is possible to detect (Creel, Aslin, \& Tanenhaus, 2008; Mitterer \& Reinisch, 2013).
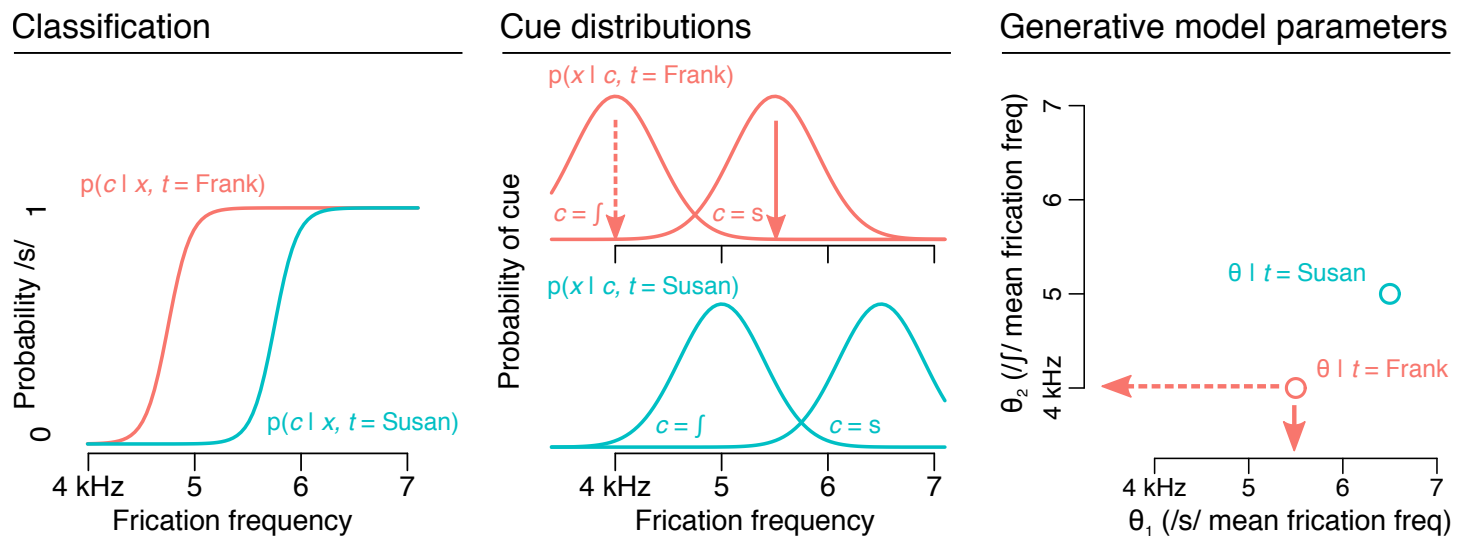

Figure 2.17: Talker-specific speech perception can be formalized as inference under uncertainty conditional on talker identity. The probability that a particular cue value $x$ (here, frication frequency) was intended to be category $c(/ \mathrm{s} /$ or $/ \delta /)$ depends on the talker $t$ that produced it, written $p(c \mid x, t)$ (left). This probability is related via Bayes Rule to the talker-specific likelihood, the distribution of cues produced by talker $t$ for each category, $p(x \mid c, t)$ (middle). These distributions can be described by the parameters of the generative model, $\theta$, such as the talker's mean frication frequency for $/ \mathrm{s} /$ and $/ \mathrm{J} /$ as plotted (right). Although we only plot two parameters, many more are required to even approximate the full generative model. Each talker can be thought of as a point in this (very high dimensional) parameter space.

\section{How does the ideal adapter formalize talker-specificity?}

We can formalize the intuition that listeners use talker-specific information to guide speech perception within a Bayesian inference framework in the following way (visualized in Figure 2.17). Speech perception is treated as an inference process, where the listener is trying to infer the talker's intended category $c$ (or message, more generally), given some acoustic observation $x$. As discussed in Part I, this relationship is probabilistic, due to unavoidable variability in pro- 
duction and sensory uncertainty, and we can express the cue-to-category inference using Bayes' rule, as in Equation (2.1), as a combination of how likely a category is a priori (the prior), and how well it predicts the observation (the likelihood). Talker-specific information can come into play in both the prior and the likelihood. If a talker is more likely to produce /s/ (overall or because of lexical preferences), this should be taken into account in the prior probability assigned to $/ \mathrm{s} /$. More importantly, given that each talker might produce a different distribution of cues for /s/, the likelihood of any given observation also depends on the talker. ${ }^{22}$ Treating both the likelihood and prior as conditional on the talker (denoted $t$ ) results, after applying Bayes rule, in a talker-specific posterior over possible intended categories:

$$
p(c \mid x, t) \propto p(x \mid c, t) p(c \mid t)
$$

This is shown schematically for classifying a VOT value as /s/ or $/ \mathrm{f} /$ in Figure 2.17: different talker-specific cue distributions (middle) result in different category boundaries (left).

However, this way of formalizing talker-specificity doesn't exactly capture the fact that the likelihood $p(x \mid c, t)$-the distribution of cues for each category-depends on the talker's generative model, rather than the talker's identity per se. We can represent a particular generative model with a vector of its parameters - things like the mean VOT for $/ \mathrm{b} /$, the variance of the frication frequency for $/ \mathrm{s} /$, etc.- - jointly denoted as $\theta .{ }^{23}$ If the listener knew, exactly, the

\footnotetext{
${ }^{22}$ The belief-updating model implemented in Part I only models changes in the likelihood.

${ }^{23}$ We represent the generative model via a list of its parameters as a notational convenience, and not to make any claims about whether or not the listener's actual generative model is a parametric model, where each category is, for instance, a normal distribution over its cues. Formally, we simply mean the parameters $\theta$ to be a description of the generative model, which might be as compressed as a vector listing the mean and variance of each category on each cue dimension, or as fine-grained as an infinitely long vector which just lists the actual likelihood assigned by each category to each possible sound.
} 
talker's actual generative model, then we could write the talker-conditional likelihood as directly conditional on that actual generative model:

$$
p(x \mid c, t)=p\left(x \mid c, \theta=\theta_{t}\right)
$$

Of course, we have argued above that listeners in general do not have direct access to the exact generative model, but rather some uncertain beliefs about it. That is, the listener's knowledge about the talker $t$ 's generative model is better described as a probability distribution, $p(\theta \mid t)$, rather than a single value, $\theta_{t}$ (Figure 2.17, right). One consequence of this is that the talker-specific likelihood should, ideally, take into account this uncertainty by averaging the likelihood assigned by each possible generative model $p(x \mid c, \theta)$, weighted by how likely the listener thinks that particular generative model is for talker $t, p(\theta \mid t):{ }^{24}$

$$
p(x \mid c, t)=\int p(x \mid c, \theta) p(\theta \mid t) \mathrm{d} \theta
$$

Making beliefs about the generative model parameters explicitly conditioned on talker identity introduces another level of structure above and beyond the belief-updating model in Part I. That model simply sought to infer the appropriate generative model parameters based on some, overall beliefs about what parameters were likely overall-the prior $p(\theta)$-and how well the current speech was explained by each possible generative model-the likelihood $p(x \mid \theta)$. The belief-updating model from Part I (implicitly) thus assumes that the same prior beliefs would be relevant, no matter the situation, and all that it can do to adapt to the current situation is to update its beliefs through distributional learning. This makes the incremental belief updating model and other flat learning models—such as simple recurrent networks,

\footnotetext{
${ }^{24}$ This further assumes that the listener recognizes talker $t$ with complete certainty. In many cases, this is a reasonable simplifying assumption, but in many others it is not, and we will address the consequences of this in a later section.
} 
(Chang et al., 2006; Elman, 1990), other connectionist models without further assumptions, (Mirman et al., 2006; Lancia \& Winter, 2013), non-hierarchical reinforcement learning, (Gallistel et al., 2001), etc.-insufficient to account for some of the most basic properties of speech perception.

However, if listeners have prior beliefs that are specific to-conditioned on-a particular familiar talker, then they do not need to bring exactly the same beliefs to every situation. Rather, by recognizing a familiar talker they can deploy the corresponding talker-specific prior beliefs to "adapt" to the current situation without needing to actually go through the process of distributional learning. In the next section, we will explore the question of how listeners get to the point of having beliefs about a particular talker's generative model, but first we discuss what we see as some of the most pressing issues for future work that this raises.

\section{Open questions: talker-specificity}

One of the consequences of thinking about talker-specific beliefs as distributions over generative models is that the inevitable uncertainty of these beliefs might lead to further uncertainty in how speech is classified, above and beyond the inherent uncertainty from the probabilistic nature of speech perception itself. As a result of having only uncertain beliefs about a talker's generative model, a truly ideal adapter would have more uncertainty about how to categorize a particular cue value when they are less certain about the talker's generative model. This could result in, for instance, shallower category boundaries than predicted by a true ideal listener with perfect knowledge of the generative model. We should stress here that maintaining uncertainty is not per se a bad thing. To the contrary, properly accounting for uncertainty about the talker's intended message is a strength of this approach, and it would be far worse to be overly-confident about a classification that turns out to be wrong. Under this 
view, because listeners should have less uncertainty about highly familiar talkers, classification behavior should be closer to what is predicted by the actual cue distributions. Alternatively, listeners might not take into account their full uncertainty in the generative model, and go with their best guess rather than averaging over the full distribution. Other intermediate approximations are possible, with the listener considering only a finite number of different possibilities. While processing of familiar talkers is overall faster and more accurate, we do not know how this is reflected in the detailed patterns of categorization behavior, which is required to effectively evaluate how much uncertainty listeners are maintaining about the current generative model. Working out the implications of these various ways of dealing with uncertainty is thus in our view a pressing topic for future work, both computational and behavioral.

Another question is whether talker-specific beliefs about the generative model are something that is unique to speech perception, or instead reflects more general perceptual strategies. That is, here we have focused on talker-specificity. However, the ideal adapter framework in principle predicts that listeners might form beliefs about any type of re-encountered situation/context, if these context/situations are reliably associated with systematically different speech statistics (generative models) and encountered sufficiently frequently. This could include beliefs about being in certain types of spaces (an echoey cathedral, a wide open field, a room with a light that buzzes at particular frequency, etc.). Of course, many of these properties are relevant to many other sorts of auditory processing, like sound source localization, and the use and deployment of context-specific beliefs about cue statistics might not be something that is unique to speech processing. Rather, they may reflect a general property of both higher- and lower-level sensory processing, so that the relevant inference might occur below the levels we have so far considered. 


\subsubsection{Learning talker-specificity through adaptation}

So far, we've discussed the benefits of talker-specific knowledge (and how it can be formalized in the ideal adapter framework) assuming that the listener is familiar with some talkers and their particular cue statistics (generative models). But of course listeners start off knowing very little about a particular talker's generative model, and so in order to be able to make use of talkerspecific cue statistics, listeners need to learn about those statistics from experience. In Part I, we discussed evidence that listeners rapidly adapt to cue statistics in a novel situation, as predicted by the ideal adapter faced with the lack of invariance. However, if a listener only adapted, then they would never be able to develop the sort of talker-specific representations that are now generally accepted to be a basic feature of the speech perception system. The ideal adapter framework thus also predicts that, when faced with the need to learn such talker-specific representations, listeners do not just adapt in the short term, but hold onto their updated beliefs about the generative model for whatever talker (or kind of situation, more generally) that they have adapted to.

\section{What do we know about talker-specific adaptation?}

Despite being very rapid, phonetic adaptation has been found to lead to stable, persistent representations. This suggests that rapid phonetic adaptation is, in many cases, better thought of as the result of listeners learning something about the talker they encounter in the experiment. A prime piece of evidence for this is that the effects of phonetic adaptation can remain strong even after intervening exposure to speech from another talker (Kraljic \& Samuel, 2005, 2007), even after leaving the laboratory and returning 12 hours later (Eisner \& McQueen, 2006). ${ }^{25}$ Moreover, there's also some evidence that listeners can learn separate

\footnotetext{
${ }^{25}$ While there are some situations in which intervening speech from another talker does disrupt adaptation (e.g. the /d/-/t/ condition in Kraljic \& Samuel, 2007), this is broadly consistent
} 
generative models for two different talkers in the same situation, even when utterances from the two talkers are mixed together (C. M. Munson, 2011). This would not be possible if listeners were simply tracking the short-run statistics of particular acoustic cues like VOT.

\section{How does the ideal adapter formalize talker-specific adaptation?}

In the ideal adapter framework, this sort of talker-specific adaptation follows naturally from the idea, introduced in the last section, that listeners are using talker-specific beliefs about the generative model. Talker-specific beliefs about the generative model are updated in a similar way as in the "flat" beliefupdating model introduced in Part I: by bringing the cue statistics predicted by the generative model into better alignment with the observed cue statistics. The only difference is that instead of considering the statistics of all observations (as the belief-updating model of Part I implicitly does), the listener should consider only the statistics of observations produced by a particular talker in updating their beliefs about that talker's generative model.

Such talker-specific belief-updating is formalized in generally the same way as it was in the belief-updating model in Part I. For each observation $x$ from talker $t$, the listener updates their uncertain beliefs about talker $t^{\prime}$ 's generative model, $p(\theta \mid t)$, by combining them with information about which generative models are more or less compatible with the observation $x:^{26}$

$$
p(\theta \mid t, x) \propto p(x \mid \theta) p(\theta \mid t)
$$

with the more general prediction of the ideal adapter that listeners are tracking the overall distribution of generative models across situations, which we discuss below.

${ }^{26}$ Really, the listener's inference about the intended category depends on their inference about the generative model parameters, and vice versa; see Equation (2.5). Here we average over (marginalize out) possible category interpretations of $x$ for simplicity's sake, as in Equation (2.7). 


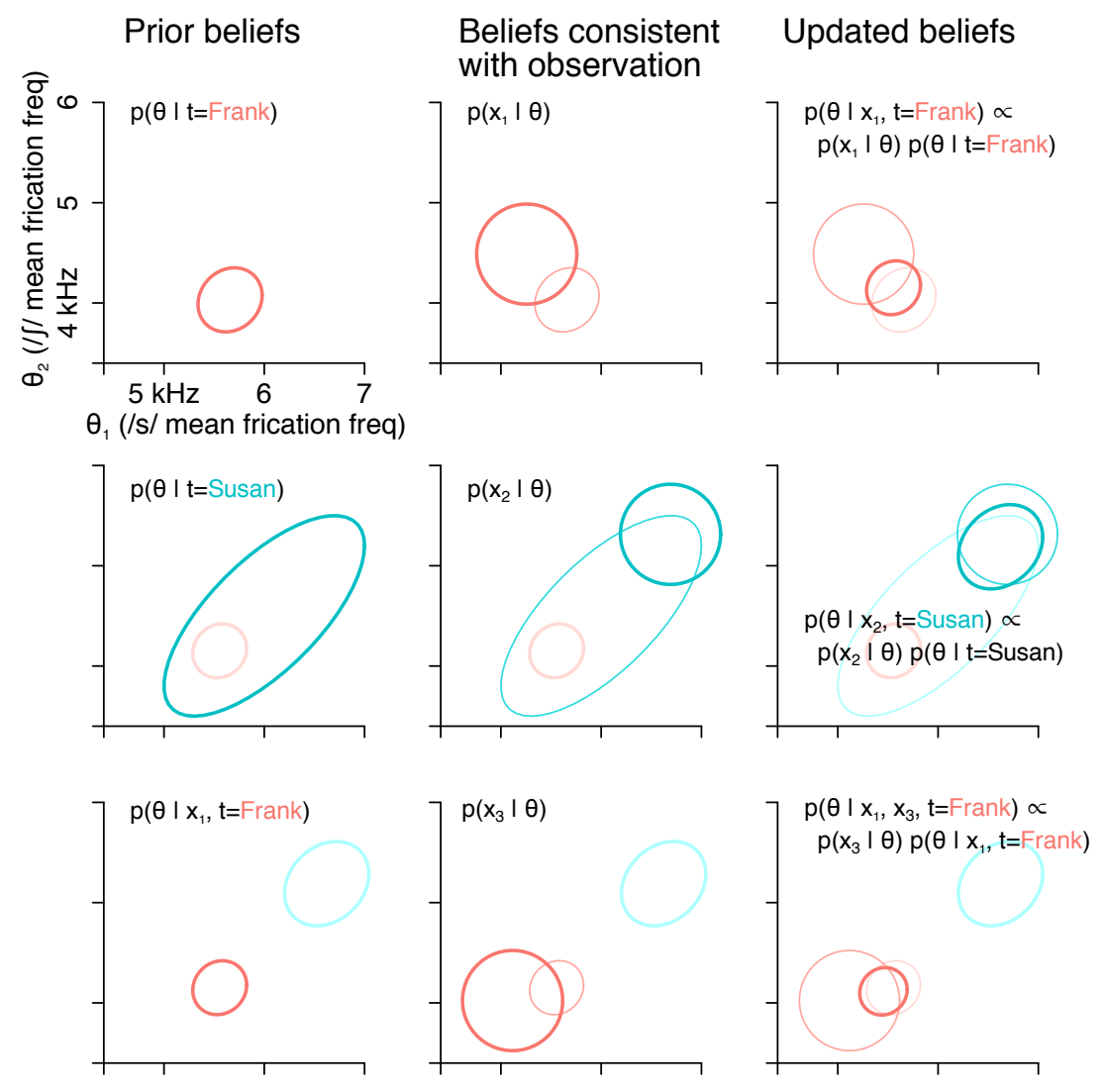

Figure 2.18: Adapting to multiple talkers: Updating of talker-specific beliefs allows listeners to continue to learn about individual talkers' generative models by accumulating evidence over multiple encounters. These plots visualize beliefs about generative models as distributions in generative model parameter space (here, showing only mean frication frequencies for $/ \mathrm{s} /$ and $/ \mathrm{J} /$ ). Each panel plots equiprobability contours of a distribution (that outline the highest probability region of generative model parameter space), based on prior experience (left column), a single observation (middle), and their combination after belief updating (top). Top row: updating beliefs about a familiar talker ("Frank"), starting from relatively specific beliefs (left). A single observation $x_{1}$ is compatible with a wide range of generative models (middle), but when combined with prior beliefs leads to more specific updated beliefs. Middle row: updating beliefs about a new talker ("Susan"), encountered next, based on very vague prior beliefs and the next observed speech $x_{2}$. Bottom row: continuing to update beliefs about Frank-which are not affected by the intervening speech from Susan-after observing another cue value $x_{3}$.

Note that, as in Equation (2.13), the dependence on the talker is entirely driven by the talker-specific beliefs about the generative model; given a particular 
value of the generative model parameters, the talker's identity doesn't change the likelihood of an observation $x$. These updated beliefs can then be further updated with other observations, with the updated beliefs after the first observation $x_{1}$ from talker $t$ serving as the starting point for updating beliefs after hearing another observation from talker $t, x_{2}$ :

$$
p\left(\theta \mid t, x_{1}, x_{2}\right) \propto p\left(x_{2} \mid \theta\right) p\left(\theta \mid t, x_{1}\right)
$$

In this way, the ideal adapter can combine information from multiple encounters with talker $t$, even if they are separated by intervening speech from other talkers (Figure 2.18). Combining information from different encounters with a single talker is important for inferring accurate beliefs about that talker's generative model because these generative models are extremely complex, with many different phonetic categories, each of which is cued by multiple acoustic features whose statistics have to be tracked. Each observation thus only provides a little bit of information about the talker's whole generative model, and arriving at accurate beliefs with low uncertainty requires, in principle, quite a bit of evidence. While this point might explain why listeners continue to update talker-specific beliefs over multiple encounters (preliminary evidence for which is provided by C. M. Munson, 2011), it raises the question of how listeners achieve reasonably robust speech perception even for relatively unfamiliar talkers. Besides the speech they directly observe, listeners have another powerful source of information: the range of different generative models they have encountered in their past experience with other talkers. As we will discuss in the next section, this information - which we can formalize as the prior or base distribution over generative models-can narrow down the range of generative models that the listener needs to consider, and serve as a head start to belief updating. 


\section{Open questions: talker-specific adaptation}

One question that talker-specific belief-updating raises is what the limits on talker-specificity in adaptation are, both in the short term and over the long term. In the short term, how many sets of beliefs can a listener simultaneously maintain and update? There is fairly good evidence that listeners can maintain at least two distinct sets of beliefs for novel talkers in the same context (C. M. Munson, 2011; Kraljic \& Samuel, 2007). Talker-specificity has been observed in recognition memory for words for up to 10 talkers at a delay of one day (Goldinger, 1996). But for more subtle effects like sublexical recalibrationchanges in category boundaries that generalize to words not already heardit's not known how many talkers can be tracked. We hope that future work will address how the number of talkers, and the overall similarity of their cue distributions, affects talker-specific adaptation.

It's also not known what the limits on talker-specific adaptation are in the long term. Do listeners always create a distinct, persistent set of beliefs for each new talker? If the listener has good reason to think that they will not meet the talker again, then it may make sense to adapt and then forget. But this would be difficult to measure in a laboratory experiment, because re-testing listeners after any appreciable delay requires a degree of logistical coordination that provides pretty good evidence that persistent representations will be useful. Moreover, it may in some cases be more efficient to group multiple, highly similar talker together, which is addressed in the following section.

\subsubsection{Generalizing across talkers}

An obvious question is where this belief updating process starts for a novel talker. In the language of the ideal adapter, what are the listener's prior beliefs when first encountering an unfamiliar talker? Before hearing any of a novel 

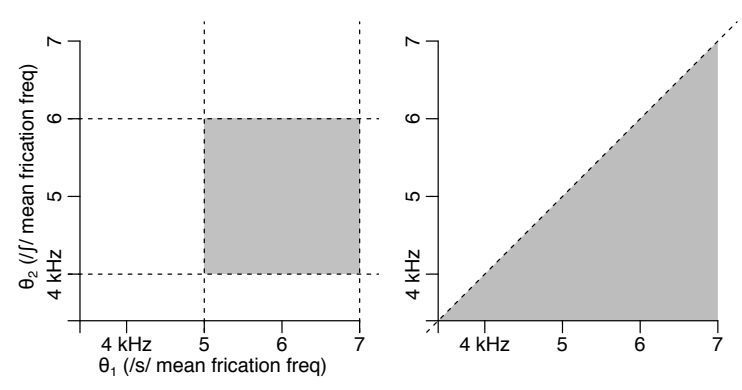

Figure 2.19: Two examples of how prior experience with many talkers of a language can constrain the space of generative models that has to be searched during adaptation. Left: an individual talker's mean frication frequency for $/ \mathrm{s} /$ generally falls in the $5 \mathrm{kHz}$ to $7 \mathrm{kHz}$ range, while the mean for $/ \mathrm{J} /$ is typically in the $4 \mathrm{kHz}$ to $6 \mathrm{kHz}$ range. Combined, these exclude much of the logically possible space of generative models. Right: moreover, the mean for $/ \mathrm{s} /$ is generally higher than the mean for $/ \mathrm{J} /$, which also excludes a substantial proportion of the possible generative models.

talker's speech, the only thing that the listener has to go on is their previous experience with other talkers. To the extent that there's structure to this experience that can benefit adaptation to a novel talker, it behooves the listener to take advantage of it, by generalizing their experience with similar talkers. At the highest level, all talkers of the same language are by definition similar, and thus one way in which experience with different talkers can help listeners is that it provides some information about the overall range, or distribution, of generative models that exist in the world, and hence that the listener should be prepared to expect from new talkers in the future. By picking up on the structure of previous experience in this way, listeners can get a head start in adapting to a new talker, because their previous experience can tell them that some types of generative models are overall more likely than others. For instance, across talkers of American English, a particular talker's mean /s/ frication frequency centroid (an important cue in distinguishing fricatives) generally falls in the range of $5 \mathrm{kHz}$ to $7 \mathrm{kHz}$, and the mean $/ \mathrm{J} /$ frication centroid typically falls in the range of $4 \mathrm{kHz}$ to $6 \mathrm{kHz}$ (Newman et al., 2001). This means that a listener can (probabilistically) rule out generative models where $/ \mathrm{s} /$ or $/ \mathrm{J} /$ have mean 
frication centroid outside these ranges, which substantially narrows down the range of generative models that they have to initially consider, providing a head start to adaptation (Figure 2.19). There are further regularities at more specific ways of grouping talkers. For instance, female talkers tend to produce both $/ \mathrm{s} /$ and $/ \mathrm{f} /$ with relatively high frication frequencies, while males tend to produce them with relatively low frequencies. Thus, knowing something about indexical variables - who is talking, and what kind of person they are-makes some of the variability in generative models predictable.

Moreover, there are regularities across talkers in the relationships between generative model parameters that a listener can take advantage of to give a further head start to adaptation. For instance, even though the range of mean centroids for $/ \mathrm{s} /$ and $/ \mathrm{J} /$ overlap across talkers, within a talker the mean centroid frequency for /s/ is almost always higher than for $/ \int /$ (Newman et al., 2001). Based on this information listeners can also (again probabilistically) rule out generative models where the relative mean centroid of $/ \mathrm{s} /$ and $/ \mathrm{f} /$ is reversed from the typical pattern. This sort of structure essentially cuts in half the number of generative models that need to be considered, a priori (Figure 2.19).

Constraints like these make the problem of adapting to a new talker, in principle, vastly easier than learning the language in the first place. The overall space of generative models that are likely to occur for a particular language or group of talkers is much more restricted than the space of generative models that occur across all talkers of all languages. Some of these constraints might be innate (and common, at least probabilistically, to the world's languages), but other obviously have to be learned for each particular language (like the range of mean VOTs allowed for each category Lisker \& Abramson, 1964).

\section{What do we know about how listeners generalize across talkers?}


Generalizing across all talkers First, there is some tentative evidence that listeners are sensitive to the overall range of generative model they have encountered. If listeners are sensitive to this range, then listeners who have experience with a broader range of generative models should, on average, be better prepared to adapt to unusual speech. Baese-berk et al. (2013) found that, as predicted, listeners who had to transcribe sentences from four talkers with four different foreign accents were more accurate on a fifth talker, with a fifth accent, relative to listeners who only heard a single accent (distinct from the test talker's).

Conversely, listeners also use the range of generative models they have previously encountered to narrow down the hypotheses they consider for a new talker. It follows that it should be harder to adapt to talkers whose generative models fall outside the typical range. This prediction is borne out in recent work. For instance, Idemaru and Holt (2011) exposed listeners to various combinations of two cues to a voicing contrast (e.g. /b/ vs. /p/), VOT and fundamental frequency (f0). Canonically, these two cues are positively correlated within a talker: higher VOTs occur with higher f0s, and correspond to voiceless stops like $/ \mathrm{p} /$. While uncorrelated VOT and $\mathrm{f} 0$ are rarer, they are also observed. Crucially, anti-correlated VOT and $\mathrm{f} 0$ are generally not observed. If listeners have implicit beliefs that reflect these correlations, this should make it harder to adapt to unnatural talkers for which the two cues are anti-correlated. This is indeed what Idemaru and Holt (2011) found: listeners were able to adapt to a two-dimensional distribution of VOT and $\mathrm{f} 0$ where $\mathrm{f} 0$ was uncorrelated with VOT. However, listeners were not able to fully adapt to a distribution where VOT and f0 were anti-correlated. Similarly, Sumner (2011) found that American English listeners were not able to adapt to a talker who always produced voiced stops (i.e. $/ \mathrm{b} /, / \mathrm{d} /, / \mathrm{g} /$ ) with substantial prevoicing (negative VOTs). Given that American English talkers typically produce these sounds with VOTs 
of $0 \mathrm{~ms}$, this inability to adapt, too, might be a consequence of cross-talker generalizations based on prior beliefs.

Generalizing based on social group membership Listeners often know (or can infer) more about a talker than that they are a speaker of English (or whichever language). To the extent that different a particular group of talkers systematically differs in their generative models from talkers in general, the listener can benefit by identifying whether a talker is a member of this group, and using their previous experience with this group to provide an even bigger head start to adaptation. For instance, there are dramatic differences in how men and women produce many phonetic categories (e.g., Hillenbrand et al., 1995; Jongman et al., 2000; McMurray \& Jongman, 2011; Newman et al., 2001), and thus the listener might (probabilistically) rule out a range possible generative models, further facilitating fast adaptation. ${ }^{27}$

One straightforward prediction of this is that in the absence of enough direct experience with a talker to directly converge on that talker's generative model, the listener's beliefs will be a combination of their (gender-specific) prior beliefs and whatever they manage to glean from what speech they have observed. For speech sounds that differ systematically between male and female talkers, such as fricatives, the listener's best guess about the appropriate generative model for the current speech is expected to depend on whether the listener believes the signal to stem from a male or a female talker. If, as we have argued above, listeners use these beliefs about the generative model to guide interpretation of speech sounds, then changing the perceived gender of the talker should change categorization (e.g., by displaying a picture of a male vs. female face along with

\footnotetext{
${ }^{27}$ Of course, as above, if the talker's generative model is actually outside the normal range for women, this would hurt adaptation. But, by definition, the distribution of the generative models for talkers in a particular group captures most of the talkers in that group, and so on average will be beneficial.
} 
the audio stimulus) .

This prediction is born out in a study by Strand and Johnson (1996) (see also Strand, 1999; Benjamin Munson, 2011). Listeners heard sounds on an $/ \mathrm{s} /-/ \mathrm{J} /$ ("sod"-"shod") continuum, made by varying the frication frequency from high (/s/-like) to low (/J/-like), paired with either a male or a female face. Frication frequencies are typically lower overall for male talkers than for female talkers (Jongman et al., 2000; McMurray \& Jongman, 2011; Newman et al., 2001), If listeners take this information into account then they should place the $/ \mathrm{s} /-/ \mathrm{J} /$ boundary at a higher frequency if they think the talker is female, resulting in fewer /s/ responses. This was exactly what Strand and Johnson (1996) found. Analogous results have been found using vowels (Johnson et al., 1999) and using different vocal sources as cues to gender (rather than faces Strand \& Johnson, 1996; Strand, 1999; Johnson et al., 1999; Benjamin Munson, 2011).

Beyond gender, there is evidence that listeners also use their experience with the speech of different social groups as a source of information about where to start adaptation. In a vowel matching task where they were told that the talker was from Canada, listeners from Detroit, MI chose tokens with more Canadian raising as matching than when they were told the talker was from Detroit (Niedzielski, 1999). This suggests that listeners' perception of these vowels was biased towards what they expected to hear from a Canadian talker. There is, more generally, a growing literature on the extent to which perceived social group membership affects language comprehension (Drager, 2010; Hay, Warren, \& Drager, 2006; Hay \& Drager, 2010; Staum Casasanto, 2008; Sidaras \& Nygaard, 2014).

\section{How does the ideal adapter formalize generalization across talkers?}

In the ideal adapter framework, the listener's beliefs about generative models are formalized as distributions over generative models, which assign more or 

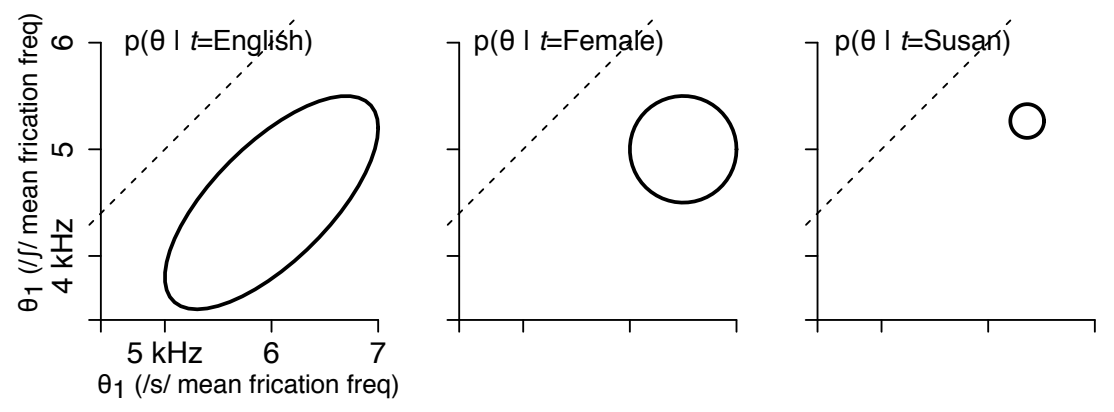

Figure 2.20: Prior beliefs at varying levels of specificity, formalized as conditional distributions over generative model parameters (plotted as ellipses of most probability mass). Left: Experience with all talkers of English constrains the listener's prior beliefs about another talker of English, but only very broadly: the mean frication centroid for $/ \mathrm{s} /$ tends to be higher than for $/ \mathrm{J} /$, and they tend to be positively correlated, but the actual values can range quite a bit. Middle: experience with all female talkers provides more information, because female talkers tend to pronounce $/ \mathrm{s} /$ and $/ \mathrm{J} /$ with frication frequency means on the high side of the overall range. Thus the distribution of females' generative model parameters for $/ \mathrm{s} /$ and $/ \mathrm{J} /$ is more concentrated than that of all English speakers. Right: experience with a particular female talker provides even more information, and the corresponding distribution over generative model parameters is even more concentrated

less probability to each possible generative model. This is true both for beliefs about a particular talker's or situation's generative model, like $p(\theta \mid$ Susan). We can also formalize beliefs about what generative models are likely for any talker of the language $-p(\theta \mid$ English $)$-or for a female talker $-p(\theta \mid$ female $)-$ or a Canadian talker $-p(\theta \mid$ Canadian $)$. When first encountering a novel talker, all the listener has to go on are these sort of beliefs about the distribution of generative models across individual talkers in some group.

Above, we have talked intuitively about how the listener's experience with the generative models of different talkers from a novel talker's language or social group can rule out a lot of generative models ahead of time and give a head start to adaptation. In the ideal adapter framework, this is a consequence of thinking about belief-updating as statistical inference, where the distributions over generative models corresponding to the listener's prior beliefs 
and the information from the speech they observe are combined according to Bayes rule. In order to converge on the particular generative model for the current situation, the listener has to allocate more and more probability mass to it. Consequently, the more probability is allocated to it ahead of time, the less information is required from observations.

The reason that more specific (or constraining) prior beliefs provide more of a head start is that by definition, probability distributions have to add up to one, and so spreading probability mass over a larger range of generative models means that less probability mass is allocated to each particular possible generative model. Thus, conversely, the more generative models that the listener can exclude a priori, the more probability they can allocate to each remaining generative model. If the actual generative model for the situation lies within this range, then fewer consistent observations are required in order to reach a particular level of probability for the actual current generative model.

The price that is paid for this head start is that more specific prior beliefs are less flexible. That is, if the prior beliefs are wrong and the actual generative model lies outside the range that has non-trivial prior probability, then much more evidence will be required to reach the same level of belief in the actual generative model. ${ }^{28}$ This is why, in principle, the listener should only use specific beliefs when they are fairly certain that they are applicable. While this might seem like a problem for the ideal adapter framework, in the next section we will show that the ideal adapter framework also points towards a solution, which is to treat the problem of identifying which beliefs are applicable as another inference problem, which can be solved by combining top-down information (like visual recognition of a particular talker) with bottom-up information from the speech itself.

\footnotetext{
${ }^{28}$ This is really a consequence of Bayes rule assigning posterior probability as the product of prior probability and likelihood. That means that what "adds up" is actually log-probability, which goes to negative-infinity as probability goes to zero.
} 


\section{Open questions: Generalization across talkers}

How do listeners know who to group together? In order to make use of structure in the distribution of generative models at different levels-from talker-specific to groups of talkers to the overall base distribution-listeners have to somehow pick up on and learn this structure. There is some evidence that people can not only induce this sort of structure, but in some cases can do so surprisingly quickly. Bradlow and Bent (2008) had listeners transcribe sentences from accented talkers. In the same talker condition, the sentences were all produced by a single accented talker, and listeners transcribed sentences from this same talker more accurately in a later test phase. In the multiple talker condition, listeners transcribed the same total amount of speech, but from four different talkers (distinct from the test talker). Despite the test talker being novel to them, listeners in the multiple talker condition showed the same benefit of experience as the same talker group. Listeners in a single other talker condition did not receive any benefit to exposure to a single accented talker when tested on the novel test talker, which suggests that listeners in the multiple talker condition have extracted some accent-level beliefs after a relatively small amount of exposure (on the order of an hour or two).

In the language of the ideal adapter framework, we might say that listeners need to impute structure to the variability in the generative models they have experienced. Much like Bayesian models of category learning during language acquisition (e.g. Feldman, Griffiths, Goldwater, \& Morgan, 2013; Perfors, Tenenbaum, \& Regier, 2011), this structure induction can be thought of as another problem of inference under uncertainty. Each way of grouping previously encountered generative models is an imputation of some kind of structure, and each possible grouping may be more or less compatible with previous experience and a priori expectations about how likely particular sorts of groupings are. However, the ideal adapter framework, as articulated here, has very little 
specific to say about how this inference might work - even at a computational level—outside of very general predictions (e.g. simpler groupings are naturally preferred because of the Occam's razor property of Bayesian inference; Perfors et al., 2011; MacKay, 2003, pp. 343-356).

How are multiple, overlapping groups represented? Each individual talker can belong to many different groups of talkers, all of which may be informative about their generative model. There are many ways that such a structure might be represented formally. One of the simplest possibilities is that talkers are nested within groups ("Mandarin-accented male talkers"), which are themselves nested within more general groups ("Mandarin-accented talkers"), and so on. This has the potentially problematic feature of not allowing generalization to a combination of groups that has not directly been observed. Another possibility is that groups might be represented, not as absolute generative model parameter distributions, but as offsets from the parameters predicted based on other factors (including other group memberships). ${ }^{29}$ This would make it possible to generalize based on arbitrary combinations of features, without needing direct experience with a particular combination, but at the price of requiring the same offset for, say, male vs. female talkers regardless of their other group memberships.

These possibilities lead to at least two open questions. First, do listeners, in fact, generalize from experience with a male talker with a particular accent or dialect to a female talker of the same accent or dialect (and vice-versa)? If listeners can generalize within a gender but not across, this would suggest that they have beliefs that are specific to a particular combination of gender, dialect/accent, and possibly other indexical variables. Of course, it is possible that for some dialects/accents listeners have gender-specific beliefs, while for

\footnotetext{
${ }^{29}$ For an analogous model applied to allophonic variation of phonetic categories, see Dillon, Dunbar, and Idsardi (2013).
} 
others they do not. This leads to the second question: do males and females differ in the same way across all dialects/accents of a language? If the ways that the generative models depend on these different types of indexical variables are all independent of each other, then it makes sense to separate them out into orthogonal features. However, gender differences themselves vary quite a bit across languages (Johnson, 2006), so it is not implausible that within a language there might be dialectal variation in gender differences as well. Moreover, it is altogether possible that for some groups, gender has the same (independent effect) while for others it does not. If this is the case, then it is in those kinds of groups where gender has an independent effect that the ideal adapter predicts that listeners are most likely to generalize experience across genders. The advent of the widespread availability and use of speech recognition technology presents an exciting opportunity to gather speech from large samples of individuals, potentially allowing some of these questions about the distribution of generative models in the world to be addressed.

\subsubsection{Balancing stability and flexibility: When to adapt, gener- alize, or recognize}

Thus far, we've addressed three strategies that listeners have for dealing with the lack of invariance: rapid adaptation to totally novel talkers, recognition of familiar talkers (and the resulting talker-specificity), and generalization across groups of similar talkers. In the ideal adapter framework, all three of these strategies are the result of the listener trying to infer the appropriate generative model with differing degrees of relevant prior experience. In this way of thinking, whether speech perception is flexible-as in adaptation to novel situations-or stable-as in recognition of familiar situations-depends on how confident the listener is in their prior beliefs, which in turn depends 
on how much relevant prior experience they have. In order to introduce the relationship between these strategies and the type and amount of relevant prior experience the listener has, we have so far addressed these strategies separately, assuming in each case that the listener knows-based on non-linguistic cues-what kind of prior experience is relevant to the current situation.

While such cues are often available, they are not always available, and sometimes they do not provide high certainty. Consider, for example, the case of running into someone that one remembers from somewhere but doesn't quite remember who it is. Sometimes visual or other cues are absent, such as when picking up the phone in the absence of caller ID, or listening to a conference call with many different people. In these cases where there is some uncertainty about who is talking, there is also uncertainty about what prior experience will be most helpful in figuring out that talker's generative model (and ultimately what they are trying to communicate). When top-down cues do not unambiguously identify the talker (or type of talker), the listener can still benefit from prior experience. Specifically, if the listener can identify a familiar talker (or the group that a talker belongs to) from their speech, listeners will have nearly the same benefits of prior experience as if they had known the talker's identity ahead of time.

The ideal adapter treats the problem of whether to be flexible or stable as inference under uncertainty at yet a third level, where the listener tries to infer what type of situation they are in, and hence what prior experience will be relevant in figuring out the generative model. ${ }^{30}$ There are, as always, two sources of information that can guide this inference: the listener's top-down expectations about what type of situation they are in (like visually identifying a talker's face), and bottom-up information from the speech signal (which may be more

\footnotetext{
${ }^{30}$ The other two levels being inferring the intended category, and inferring the situation's particular generative model.
} 
or less compatible with the speech predicted by their beliefs about each possible type of situation). The ideal adapter framework predicts that listeners use these two sources of evidence in order to balance stability and flexibility on the fly.

\section{What do we know about how listeners balance stability and flexibility?}

Balancing stability and plasticity requires that listeners deploy the most informative prior experience that is relevant in each situation. This is true whether or not they know a priori what prior experience is relevant. Thus, we argue, balancing stability and plasticity in speech perception requires that the listener

dynamically combine top-down information about what kind of situation they are in with bottom-up information from the speech signal. However, little is known about how listeners trade off these two sources of information. What little we know suggests that listener do, in fact, use both bottom-up and topdown information to infer the type of situation they are in and what prior experience is currently relevant. Based on these inferences, listeners can then determine how flexible to be in the current situation. Evidence for this view is of two types, which we discuss in turn. First, listeners can recognize talkers that they are familiar with based on just bottom-up speech input. Second, listeners generalize experience with one talker to adaptation to another talker based both on the bottom-up similarity in their generative models and top-down expectations that generalization is appropriate.

Recognizing familiar talkers based on their speech Listeners use information from the speech signal itself to help infer the talker's identity (and social group membership) in a way that shapes how they interpret that talker's speech. The speech signal includes non-phonetic features like (in English) f0 range, jitter, and shimmer that distinguishes talkers (Creel \& Bregman, 2011; 
Pardo \& Remez, 2006). Listeners use this information not only to recognize familiar individuals but also to infer social group membership. For instance, we discussed above evidence that listeners change their classification of vowels and fricatives based on the gender of a visually presented face. These same studies found that synthesized vowels and fricatives-with exactly the same phonetic cue values-were also classified differently depending on the vocal source wave that was used, with stereotypically female and male vocal sources having a similar effect as a visually-presented female face (higher frequency category boundaries). Interestingly, this effect is gradient, with the effects of voices rated as non-stereotypically male or female falling in between stereotypically male or female voices (Johnson et al., 1999; Benjamin Munson, 2011; Strand \& Johnson, 1996; Strand, 1999).

Beyond non-phonetic aspects of the speech signal, the fact that different talkers use different phonetic generative models means that phonetic aspects of the speech signal can also, in principle, provide information about talker identity or group. That is, the same talker- or group-specific beliefs about the phonetic generative model which benefit speech perception once a particular talker is identified can also conversely aid in identifying a talker. To the extent that they vary across talkers-and hence benefit from talker- or group-specific representations-phonetic features like formant frequencies and VOT are by definition diagnostic of talker identity. Thus, the listener's knowledge about a familiar talker's cue statistics provides a rich source of information for identifying that particular talker based on their speech, which can in turn can affect how that same speech signal is mapped onto phonetic categories. Listeners do, in fact, take advantage of such phonetic information for talker recognition (Creel \& Bregman, 2011; Pardo \& Remez, 2006), and can explicitly recognize talkers with very high accuracy based on sine-wave (Remez, Fellowes, \& Rubin, 1997) or noise vocoded speech (Sheffert et al., 2002). Such processing of 
speech removes all of the fine spectral detail (and much if not all of the voice quality information) but preserves enough phonetic information to allow for reasonably good comprehension.

Generalizing experience with one talker to another, or not When there is uncertainty about what prior experience is relevant, information from the speech signal helps processing of novel talkers in two ways. First, in this paper we have generally simplified our discussion by focusing on a few phonetic features. However, in everyday speech perception the generative model is highly complex, covering many categories, each cued by many acoustic features. Somewhat paradoxically, when we consider that the listener must also infer the kind of prior experience that is relevant, this makes their job easier: even from a small amount of speech from a novel talker, listeners can in all likelihood recognize that none of their talker-specific representations provide a good match to the current situation. Second, even a small amount of information about the generative model can help identify whether, and which, more informative group-specific beliefs might be relevant, meaning that listeners have to fall back on the least informative beliefs only as a last resort. This is important because on the one hand, even a little bit of experience with one or more similar talkers provides a big boost to adaptation, but on the other hand, mistakenly assigning a talker to a particular group actually makes it harder to adapt to their actual generative model, as discussed in the last section. If listeners are in fact continuously trying to identify what, if any, familiar group a novel talker belongs to based on the speech they produce, then they should flexibly generalize from experience with one talker to another when it's warranted (by top-down expectations and bottom-up similarity) but adapt in a talker-specific way when it is not.

This is an important though comparatively understudied issue. A series of 
recent studies provides some tentative evidence for the predictions of the ideal adapter framework. These studies looked at how much listeners generalize perceptual learning for a male talker to a different, female talker (or vice versa; Eisner \& McQueen, 2005; Kraljic \& Samuel, 2005, 2006, 2007). In one version of these experiments listeners hear a male talker's production of a sound ambiguous between a $/ \mathrm{s} /$ and a $/ \mathrm{J} /$, embedded in a word that indicates it was intended to be an $/ \mathrm{s} /$. After exposure, listeners are tested on two different /s/-/S/ continua: one produced by the same male talker as exposure, and the other produced by a different (female) talker. In the case of $/ \mathrm{s} /-/ \mathrm{J} /$ (and $/ \mathrm{s} /-$ $/ \mathrm{f} /$ ), listeners show the expected recalibration effect-more /s/ responses after /s/ exposure-when tested on the same talker, but no effect when tested on the different, female talker (Eisner \& McQueen, 2005; Kraljic \& Samuel, 2005). However, in another version of the experiment using a /d/-/t/ contrast, listeners show the same recalibration effect on both the same- and different-talker test continua (Kraljic \& Samuel, 2006). In such experiments, the only information that the listener has about the unfamiliar female test talker comes from the test stimuli themselves. In the case of a voicing contrast like /d/-/t/, the overall distribution of the critical cue-VOT—is generally similar between the familiar male and unfamiliar female talker, and so the test stimuli provide information that these two talkers' generative models are overall quite similar, at least in terms of VOT and the voicing contrasts it cues. If listeners are using this information, then they should apply what they have learned about the male talker to the unfamiliar female talker, which would lead to the generalization observed by Kraljic and Samuel (2006). Conversely, the range of cues in the $/ \mathrm{s} /-/ \mathrm{f} /$ male and female continua used by Kraljic and Samuel (2005) differ quite dramatically: the highest spectral centroid of the male talker's /s/ is still lower than the lowest $/ \mathrm{J} /$ end of the female talker's continuum. Thus, the test stimuli from the female test talker provide evidence that these two talkers are quite different in 
how they produce this contrast, and thus it does not make sense to apply what has been learned about the male talker to the unfamiliar female test talker.

Kraljic and Samuel (2007) present evidence that listeners use both their top-down expectations about whether two talkers should be grouped and the bottom-up similarity of their cue statistics to guide not just processing of a totally unfamiliar test talker, but also to guide adaptation to multiple talkers. When exposed to both a male and female talker, whose ambiguous pronunciations are disambiguated in opposite ways (e.g. male as /d/ and female as /t/), listeners showed no overall learning effect between pre- and post-exposure classification of the two talker's /d/-/t/ continua, suggesting that they have tracked the statistics of these categories in a talker-independent way. But for the analogous procedure with $/ \mathrm{s} /-/ \mathrm{J} /$, classification of each talker's continuum shifted in the typical way, suggesting that they have adapted talker-specific representations (Kraljic \& Samuel, 2007).

This might be taken as evidence that listeners are simply unable or categorically unwilling to learn and maintain talker-specific beliefs about some phonetic categories. This broadly makes sense: for categories like /d/ versus / $t$ / which, in the listener's experience, show some variability across talkers but are not systematically produced differently by male versus female talkers, it is a reasonable guess that a male and a female talker encountered in the same context will produce them similarly and can be grouped together. Likewise, as noted by Kraljic and Samuel (2007), for categories that are generally produced differently by male and female talkers who are otherwise similar, it is a good bet that a male and female talker should not be grouped together.

However, if listeners are actually trying to infer whether or not two talkers should be grouped together-rather than simply relying on fixed heuristicsthen enough of the right kind of experience which contradicts these biases should be able to overcome them in specific cases. For instance, even though 
listeners tend to generalize experience with VOT distributions from a male to female talker, with sufficient experience with a male and a female talker who have different VOT distributions, listeners do in fact learn talker-specific representations (C. M. Munson, 2011). Conversely, even though listeners tend to learn talker-specific representations of fricatives, by using a modified test continuum Reinisch and Holt (2014) found that listeners can generalize experience with a female talker's fricatives to a novel male talker.

\section{How does the ideal adapter formalize the stability/plasticity trade-off?}

A mixture of prior beliefs The available evidence suggests that listeners can, on the fly, infer which type of prior experience is relevant for the current situation based on a combination of bottom-up speech information and top-down expectations. In the language of the ideal adapter framework, this in turn suggests that in situations where the listener is unsure about what type of prior experience is relevant, their prior expectations about what generative models they will encounter are a mixture of expectations given different types of prior experience. Each particular sort of prior experience can be thought of as a cluster of possible generative models, with more specific prior experience-like experience with a particular familiar talker-corresponding to highly concentrated, peaked clusters and more general experience-like with the range of generative models across all talkers in the language-being more spread out. How much each cluster contributes to the overall beliefs depends on how likely the listener thinks it is to apply in the current situation. How much the listener expects to encounter any particular generative model is thus a combination of how much it is expected given a particular set of beliefs, and how likely the listener thinks those beliefs are to apply.

One way to formalize this notion is based on representing the beliefs about generative models for a particular type of prior experience as a probability dis- 
tribution over generative models. Again, for the sake of brevity, we refer to the generative model by its parameters $\theta$, conditional on the type of prior experience $t: p(\theta \mid t)$. Here, we use $t$ to denote a more general form of talker identity, that covers beliefs ranging from particular individual talkers, like $p(\theta \mid t=$ my father), to groups, like $p(\theta \mid t=$ Boston accent), all the way to languagelevel groupings like $p(\theta \mid t=$ English speaker). Above, we assumed that the listeners knows the type of prior experience which is relevant, and thus only a single conditional distribution $p(\theta \mid t)$ is relevant. When the listener is uncertain, though, multiple different conditional distributions might be relevant. The listener's beliefs about which type of prior experience is relevant can be formalized as a probability distribution over the types $t-p(t)$-and hence their overall beliefs about the generative model parameters as a mixture of the conditional beliefs, weighted by $p(t)$ :

$$
p(\theta)=\sum_{t} p(\theta \mid t) p(t)
$$

Top-down information Let's consider the case where the listener can recognize a familiar talker — call him Frank—with high certainty based on visual information. Then, the distribution over types of prior experience $p(t \mid$ vis. info $)$ is highly peaked:

$$
p(t \mid \text { vis. info })=\left\{\begin{array}{cl}
1 & \text { if } t=\text { Frank } \\
0 & \text { otherwise }
\end{array}\right.
$$

As a result, the listeners' beliefs about the generative model parameters, given the visual information that identifies the talker as Frank, is dominated by their 
beliefs about Frank's generative model:

$$
\begin{aligned}
p(\theta \mid \text { vis. info })= & \sum_{t} p(\theta \mid t) p(t \mid \text { vis. info }) \\
= & p(\theta \mid t=\text { Frank }) p(t=\text { Frank } \mid \text { vis. info }) \\
& +\sum_{t \neq \text { Frank }} p(\theta \mid t) p(t \mid \text { vis. info }) \\
= & p(\theta \mid t=\text { Frank }) \times 1+\sum_{t \neq \text { Frank }} p(\theta \mid t) \times 0 \\
= & p(\theta \mid t=\text { Frank })
\end{aligned}
$$

That is, when the type of prior experience is known with reasonably high certainty from top-down cues, this mixture model reduces to the talker- or group-specific beliefs described in earlier sections in Part II. This formalization thus generalizes the notion of talker- or group-specific beliefs as conditional distributions. In this sort of mixture model, when top-down information about the type of situation is available, listeners can take advantage of it, which can in turn lead to effects of top-down information (like visually presented faces or other cues) changing the way that physically identical bottom-up acoustic signals are interpreted (Johnson et al., 1999; Hay \& Drager, 2010; Staum Casasanto, 2008; Strand \& Johnson, 1996). However, having a weighted mixture of different prior beliefs means that the listener does not have to rely solely on such topdown information to tell them which prior experience is relevant, but rather can also use bottom-up information.

Bottom-up information Formalizing the listener's prior beliefs about generative models in this way provides a natural way of formalizing how bottom-up, phonetic information can help infer the type of prior experience that is relevant. Inferring the type of experience that is relevant comes down to inferring the type $t$, given some observations $x$ and prior expectations $p(t)$, which we 
can think of as Bayesian inference at yet another level:

$$
p(t \mid x) \propto p(x \mid t) p(t)
$$

As usual, the listener's updated beliefs about the type of situation they are in, $t$, are a combination of their prior beliefs about the type of situation $p(t)$, and how well their prior experience with each type predicts the observations $x, p(x \mid t)$. In this formalization, observations and types are assumed to be only indirectly related, via the generative model parameters $\theta$ : each type predicts a range of generative models, $p(\theta \mid t)$, and each value of the generative model parameters predicts a range of observations, $p(x \mid \theta)$. In order to get the marginal likelihood of the signal $x$ under situation $t, p(x \mid t)$, the particular values of the generative model parameters have to be averaged out:

$$
p(x \mid t)=\int p(x \mid \theta) p(\theta \mid t) \mathrm{d} \theta
$$

The jargony term for this averaging is marginalization. And a natural consequence of marginalization is that it leads to a preference for the most specific prior experience which provides a reasonably good fit to the observed data. We can think of marginalization as essentially taking a weighted average of the likelihood of the observation $x, p(x \mid \theta)$, weighted by the type-specific probability of the generative model parameters $p(\theta \mid t)$. On the one hand, for a type of prior experience that leads to very specific beliefs-like a particular familiar talker-the predicted observations $p(x \mid \theta)$ are very similar over the range of generative models that are given reasonably high probability, and so will all either fit the data reasonably well or not. On the other hand, a type of prior experience that leads to less specific beliefs about generative models-like experience with all speakers of the language-predicts a lot of different generative models. Some of these will probably provide a good account of the observa- 
tion $x$-high likelihood $p(x \mid \theta)$-but the vast majority will not, resulting in a low average (maringal) likelihood $p(x \mid t)$.

This trade-off between specificity and accuracy goes both ways. A vague (e.g. language-wide) prior over generative models will allocate some likelihood to nearly any observation, whereas a specific (e.g. familiar talker) prior predicts only a very limited range of observations, and so assigns very high marginal likelihood to things in that range and basically nothing outside of it. So for observations that fall outside the range predicted by previous experience with specific talkers, less specific, group-level experience provide the best marginal likelihood, even if it's not (in absolute terms) very high.

Of course, inferring the type of prior experience that is relevant is only the first step to robust speech perception. Additionally, the listener also needs to infer the generative model that is best suited to the current situation. We can think of this as inference of the joint posterior distribution of generative model parameters and type of situation, $p(\theta, t \mid x)$. This combines inference about the type of situation-as discussed in this section-with situation-specific beliefupdating-discussed above:

$$
p(\theta, t \mid x)=\underbrace{p(\theta \mid x, t)}_{\begin{array}{c}
\text { situation-specific } \\
\text { updated beliefs }
\end{array} \text { situation }}
$$

Alternatively, we can think of just the overall updated beliefs about the generative model $p(\theta \mid x)$, which-like the prior beliefs $p(\theta)$-are a mixture of updated situation-specific beliefs, weighted by how likely each situation is given the observation:

$$
p(\theta \mid x)=\sum_{t} p(\theta, t \mid x)=\sum_{t} p(\theta \mid x, t) p(t \mid x)
$$


This brings us back to the central point of the second part of this paper: the ideal adapter framework reveals that we can look at recognition (and the resulting talker-specificity), adaptation, and generalization in speech perception as the natural consequence of a system that is trying to do the best that it can to comprehend speech in a variable-but structured-world. Because of the variability (or subjective non-stationarity) of the statistics of the speech signal, the listener has to adjust the generative model they use. There are two sources of information that listeners have when trying to do this: the speech they are currently processing, and their previous experience and prior expectations. Different strategies arise from the fact that in each situation, the prior experience that is relevant-if any-is sometimes more and sometimes less informative about the appropriate generative model than the current speech itself. When the listener has no relevant prior experience, the best they can do is to try to learn the generative model by adapting their beliefs to better match the recently observed speech statistics, leading to rapid adaptation. When they have prior experience that is directly relevant, like with a familiar individual talker, the best thing they can do is to rely on that prior experience, removing the need to adapt and leading to talker-specific speech perception. When they have experience that is relevant, but not directly, then the best they can do is apply that experience to the current situation and then use the actual speech statistics to converge on an appropriate generative model.

For types of experience that correspond to very specific beliefs-like a highly familiar talker-the situation-specific beliefs will change little with the addition of one more observation. If such a component for a familiar talker provides the best match to the observed speech, then the result of this process of belief updating with a clustered mixture prior is that the listener has recognized and deployed their talker-specific generative model. If, on the other hand, the best-fitting type is a very broad, general type like all speakers 
of English, then the updated beliefs are dominated much more by the actual observed data then the prior, just like when the listener knows ahead of time that they need to adapt rapidly. Similarly, if prior beliefs of intermediate specificity dominate, then the updated beliefs will reflect the observed data more than they would for a highly familiar talker, but the prior beliefs will contribute more than in the most flexible case. This is how the ideal adapter is able to flexibly deal with the lack of invariance, making the best use of different types of prior experience with different generative models. By learning about and representing the distribution of generative models in the world, the ideal adapter is better prepared to infer the appropriate generative model across many different types of situations.

\section{Open questions}

We have argued here that the ideal adapter predicts that listeners dynamically balance flexibility and stability by inferring what kind of prior experience is currently most relevant. If the listener decides that experience with a particular familiar talker is most relevant, they will show stable, talker-specific speech perception. If, on the other hand, they decide that the only relevant prior experience they have is with the full distribution of talkers of the language, then they will rapidly and flexibly adapt.

The biggest open question that this raises is how-and even whetherlisteners combine top-down and bottom-up information about what kind of prior experience is relevant. There are, in our view, at least two sides to this question. The first side is what a truly ideal adapter would do. The ideal adapter framework as currently posed addresses this only in the most general sense, saying that listeners should combine top-down and bottom-up information to determine what prior beliefs to use. But much more work is required in order to make further testable predictions. The second side is empirical, and in 
the rest of this section we review two empirical questions we see as particularly pressing.

What role do expectations of generalizability play? The studies by Kraljic and Samuel $(2005,2006,2007)$ suggest that listeners group together talkers when the distributions of cues they produce are similar. But in all these studies, the similarity of the cue distributions is confounded with the overall similarity between male and female talkers in general on the relevant phonetic dimensions: male and female talkers don't systematically differ in their VOT distributions in American English (Allen, J. L. Miller, \& DeSteno, 2003), whereas they do differ in their frication frequency distributions (Newman et al., 2001). Thus listeners may have already made up their minds about whether to group the two talkers together before hearing anything. There's some evidence that topdown expectations and bottom-up similarity can be dissociated (C. M. Munson, 2011; Reinisch \& Holt, 2014), but it remains to be seen exactly how listeners balance these two sources of information, both in the short term (e.g. over the course of a single experimental session) and over the long term (over days, weeks, or longer).

When are two generative models similar enough to group? Our discussion of how listeners might generalize experience from one talker to another on the basis of similarity of their generative models glosses over a very basic point: even if their VOT ranges are overall very similar, the whole generative model for a male talker is very different from that of a female talker. Thus, while it might make sense to lump together experience with a male and female talker for the purposes of inferring their VOT distributions, it definitely does not make sense to lump together beliefs about their vowels. One way that this dilemma could be resolved is if listeners have extracted some information 
about the variance of different parameters in groups that contain males and females (like the ad hoc grouping that might be inferred during an experiment). Properties that vary a lot across talkers within a group are not very informative about particular talkers, whereas properties that are consistent across talkers in a group can be used to constrain beliefs about individuals much more strongly. Because males and females-within most groups-vary a lot in their vowels, but not so much in their VOT, listeners can use information about the VOT distributions of individual talkers to (on average) reliably infer properties of the group, which in turn are (on average) highly informative about properties of other talkers in the same group.

An alternate possibility is that listeners have more or less separate beliefs about different "chunks" of the generative model, potentially grouping talkers differently when considering beliefs about their VOT distributions than they do when considering beliefs about vowel formants. It is not immediately obvious what-if any-divergent predictions these two accounts would make, and more work is required to understand both their computational implications and related human behaviors.

\subsubsection{Part II conclusion}

We have argued that a range of strategies that human listeners use to deal with the lack of invariance all correspond to the best that they can do in a world where generative models vary from one situation to the next, but do so in a structured way that is tied to indexical variables like talker identity and group membership. The ideal adapter framework formalizes this notion by representing situation-specific beliefs about the range (distribution) of generative models that are expected in different types of situations, from very specific (an individual talker) to very general (an entire language).Although it is beyond the scope 
of this paper to discuss in any detail, this perspective can be applied to second (and third, etc.) language acquisition as well (Pajak, Fine, et al., 2016). This range allows the listener to balance stability and flexibility, relying on prior experience when it is available and relevant, falling back on less informative but more flexible group-level beliefs. This system is not fail-safe. In order to achieve robust recognition of familiar talkers and guiding generalization across talkers, the speech perception system is forced to rely on prior experience. In highly atypical situations -which, fortunately, are bound to be rare- this very same property can slow down or prevent successful adaptation and perception (as also evident in second language learning).

Explicit structured beliefs are one way of achieving sensitivity to structured variability of cue-category mappings. However, the primary take-home message from Part II is that sensitivity to this structured variability is a critical feature of any model that seeks to explain the robustness of human speech perception. Formulating such a model and evaluating its predictions remains a task for future work.

\subsection{Part III}

We close our paper with a discussion of how the present work relates to other approaches and issues. We chose to take a computational-level approach to understanding how the speech perception system deals with the lack of invariance. Such an analysis focuses on the problem of robust speech perception. That is, our approach is guided by questions about the 'why', or the purpose of the system, the goals it typically serves, and the world it has to function in. Focusing on the 'why' of speech perception does not directly address the actual cognitive processes (and neural mechanisms) that must carry it out (Marr, 1982), but it has allowed the development of models that provide a good fit 
to human behavior, both in speech perception (Clayards et al., 2008; Feldman, Griffiths, \& Morgan, 2009; Norris \& McQueen, 2008; Sonderegger \& Yu, 2010) and in a range of phonetic adaptation phenomena (Part I of this paper). It also has allowed us to make novel predictions, some of which we have tested here and the rest of which we hope will guide future research and the development of cognitive and neural models. The ideal adapter framework is normative, in that it looks only at the in-principle constraints on performance that come from the inherent difficulty of the task and the limited information that is available in the world, without considering resource limitations. The brain is obviously not unbounded in its resources, and considerations that come from processes, representations, and mechanisms are thus relevant to future development of the framework presented here.

However, this gulf is not as wide as it might first appear. Neurally plausible algorithms exist for the sort of inferences required by the ideal adapter framework (Beck, Pouget, \& Heller, 2012; Friston, 2005; Rao \& Ballard, 1999), and resource limitations can be included in a normative framework. For instance, recent work has focused on developing "rational approximations" to rational (normative) models like the ideal adapter. These models ask what the best possible performance is under different types and levels of cognitive or neural resource constraints (Griffiths, Vul, \& Sanborn, 2012; Sanborn et al., 2010; Shi et al., 2010). For instance, one notable constraint on normative, Bayesian inference is the amount of uncertainty-or the number of different hypotheses-that can be simultaneously maintained. Listeners can apparently maintain enough uncertainty about whether or not two talkers should be grouped together to overcome initial biases about how to generalize phonetic recalibration (Kraljic \& Samuel, 2005, 2006; C. M. Munson, 2011). However, listeners also seem to prefer to stick to "first impressions" more than a purely normative model would predict (Kraljic, Samuel, \& Brennan, 2008). This suggests limits on how much 
uncertainty listeners are willing or able to maintain (see also Tzeng, Alexander, Sidaras, \& Nygaard, 2014, for related effects of presentation order when generalizing across talkers).

Likewise, process- and mechanistic-level theories do not exist in a vacuum, and computational-level considerations about the task they must perform can be relevant and informative. In this final part of the paper, we will expand on some particular ways that we think the ideal adapter framework relates to and potentially informs a range of other approaches and topics. First, a number of other approaches to the lack of invariance have been proposed, and we will briefly lay out where we think the ideal adapter framework differs from, conflicts with, and complementes these other approaches. Next, we turn to how the proposed framework, and the body of empirical literature it unites, might inform the debate about the underlying representations used by speech recognition, and the role of subphonetic detail in phonetic representations. Finally, we will discuss the possible implications for this work beyond speech perception by adult listeners to both higher-level language processing and the acquisition of phonetic categories during development.

\subsubsection{How does the ideal adapter relate to other approaches to the lack of invariance?}

Our approach is novel when compared to most models of speech perceptionBayesian and otherwise-that do not learn at all (Clayards et al., 2008; Feldman, Griffiths, \& Morgan, 2009; McClelland \& Elman, 1986; Norris, 1994; Norris et al., 2000). But we are hardly the first to consider the problem of the lack of invariance in speech perception, and a variety of other approaches exist that either directly address flexibility in phonetic categorization or address related problems. We review these in this section. 


\section{Existing models of speech perception and adaptation}

The existing models that are most directly related to our approach deal with plasticity in phonetic categorization by adding slower learning (and habituation) dynamics to existing connectionist or dynamical systems models of phonetic categorization (Lancia \& Winter, 2013; Mirman et al., 2006). In both of these models, learning is modeled with a Hebbian learning mechanism that increases the feedforward connection weight between acoustic/phonetic input units to categorical output units based on repeated co-activation. Such a learning mechanism is sufficient to qualitatively capture phonetic recalibration, but it is not clear whether the generally slow temporal dynamics of Hebbian learning can account for the very rapid recalibration effects that are typically observed (Guediche, Blumstein, Fiez, \& Holt, 2014). Furthermore, without an additional habituation mechanism (which is present in Lancia \& Winter, 2013) Hebbian learning may not be sufficient to capture selective adaptation.

At a deeper level, existing models of plasticity in speech perception arein principle or in practice-generally "flat" learners, which (in the language of the ideal adapter framework) adapt a single set of beliefs about the generative model based on recent experience. This includes connectionist models of phonetic adaptation (Lancia \& Winter, 2013; Mirman et al., 2006) as well as distributional learning models of the acquisition of phonetic categories (e.g. Feldman, Griffiths, Goldwater, \& Morgan, 2013; McMurray, Aslin, \& Toscano, 2009; Vallabha et al., 2007). Because none of these models represent the fact that the distributions (or connection weights from input to output units in Lancia \& Winter, 2013; Mirman et al., 2006) are not only situation-specific but might be encountered again after an interruption, these models cannot account for the persistence of adaptation over intervening exposure (as in Eisner \& McQueen, 2006; Kraljic \& Samuel, 2005). These models also cannot explain when we do 
or do not generalize across talkers (Kraljic \& Samuel, 2005, 2006, 2007). ${ }^{31}$ The belief-updating model presented in Part I falls into the same class of flat learner models. Thus, one of critical goals for future computational work on speech perception is to continue the development and test of the full ideal adapter framework, as we have begun to outline in Part II.

The remaining class of approaches to plasticity in speech perception is the class of episodic or exemplar models (e.g., Goldinger, 1998; Johnson, 1997a, 2006; Pierrehumbert, 2003). These models are arguably motivated by many of the same considerations that motivate the ideal adapter framework: the mapping between cues and categories is variable, in often idiosyncratic ways (Johnson, 2006), and so the speech perception system has to be sensitive to this variability. Exemplar models achieve this by storing raw acoustic traces for each exemplar that has been encountered, and categorize new inputs based on similarity to stored exemplars. By remembering every instance of a category, these models (implicitly) learn the corresponding distribution of sounds, and can achieve persistent talker-specific representations (Goldinger, 1998; Johnson, 2006). However, simply storing raw episodes alone is not sufficient to explain the ways that human listeners generalize learning on one phonetic category to unheard words (Cutler, Eisner, McQueen, \& Norris, 2010; McQueen, Cutler, \& Norris, 2006), other contrasts (Kraljic \& Samuel, 2006), and other talkers (Kraljic \& Samuel, 2006, 2007; Reinisch \& Holt, 2014). For such flexibility, some additional sensitivity to the underlying structure of the variation in cue mappings is required, both at the level of how the acoustic signal is analyzed into linguistic units (Cutler et al., 2010) and at the level of how different talkers can be grouped based on their generative models (Part II of this paper). To

\footnotetext{
${ }^{31}$ Mirman et al. (2006) accounts for the results of Kraljic and Samuel $(2005,2006)$ by using cue representations that encode the similarity or dissimilarity of the two talker's test continua. But as far as we can tell, this approach fails to predict the opposite results (C. M. Munson, 2011; Reinisch \& Holt, 2014)
} 
some extent this, too, has been recognized in recent work on episodic theories of language processing (e.g., Johnson, 2006, 2013; Pierrehumbert, 2003; van den Bosch \& Daelemans, 2013).

To illustrate this point further, we have argued (in Part II) that the speech perception system can benefit from structure in the world in how generative models vary across situations. This structure means that prior experience with the same or similar generative models can inform future adaptation and processing. We have discussed how, in the ideal adapter framework, sensitivity to this structure can be naturally formalized by structured representations, in the form of talker- and group-specific beliefs about (distributions over) generative models.

Memory-based or episodic theories provide an alternative approach which is superficially conflicting. In these approaches, prior experience is represented in an unstructured way, but sensitivity to the structure of that experience might still arise through processes by which similarity to stored episodes is computed (e.g., analogical reasoning van den Bosch \& Daelemans, 2013). This is an area of ongoing research, and it is an interesting question whether these two approaches-structure by representations or by processes-make substantively different predictions in principle. It is possible, for example, that analogical reasoning applied to unstructured memory traces can implement the sort of structure-sensitive computations we have argued for.

\section{What counts as a situation for adaptation?}

In our discussion of how generative models vary from one situation to the next, we have focused on talkers (or other indexical variables) as the main driver of this variability. The reason for this emphasis on talkers is that differences between talkers are responsible for a large amount of variability in how phonetic categories are realized acoustically (Allen, J. L. Miller, \& DeSteno, 2003; Hillen- 
brand et al., 1995; McMurray \& Jongman, 2011; Newman et al., 2001, among others). This lead us to hypothesize that listeners' beliefs about the generative model of speech need to be thought of as conditioned on their beliefs about the talker (or type of talker) they think they are listening to.

However, talker differences are not the only source of the lack of invariance, and nonlinguistic factors like background noise or general acoustics can also change from situation to situation. Additionally, the realization of phonetic categories depends on the linguistic context that those sounds are produced in (Liberman et al., 1967), and a variety of models have been proposed to account for how categorization of one segment is affected by adjacent segments (Massaro, 1987; Nearey, 1997; Nearey \& Assman, 1986; Oden \& Massaro, 1978; Smits, 2001; Sonderegger \& Yu, 2010).

This raises an important question: is what we have called a "situation" (understood as the non-linguistic aspects of the current situation like talker or setting) different from the notion of "context" in, for instance, models of coarticulation? The ideal adapter framework points towards a way of approaching this question. This is based on the underlying statistical properties of how the cue-category mappings represented by the generative model vary based on talker (and other indexical variables), linguistic context, and the combination of the two. If the effects of linguistic context are themselves sufficiently variable across talkers (as experienced by the listener), then the ideal adapter framework says that the speech perception system has a strong incentive to track category statistics as a joint function of both linguistic and non-linguistic context. If not, then they can be safely tracked separately. Even if the effects of linguistic context and indexical situation can be, computationally, treated as basically the same, it is a separate question of what the brain makes out of these two very different kinds of variability. The effects of linguistic context are relatively localized in time (only a couple of syllables), whereas indexical context is much 
more diffuse (an entire discourse, or even multiple separate encounters), and so each may impose very different demands on memory and other processing resources.

\subsubsection{What is the nature of representations underlying speech recognition?}

Even though our work here is pitched at a computational level, it does have implications for existing linguistic theories of speech recognition. By relating the lack of invariance to the strategies that people use to achieve robust speech perception-adaptation, talker-specificity, and generalization-the ideal adapter framework highlights shortcomings in the starting points for existing theories of how the speech recognition system maps cues onto linguistic categories. We will argue that the ideal adapter framework also helps to understand how recent moves away from these more extreme starting points are a step in the right direction, and-we hope-points to further productive directions for future research.

\section{Abstractionist and episodic approaches to speech recognition}

One of the persistently debated issues in speech recognition is the degree of abstraction in the representations which mediate cue-category mappings. At one extreme, abstractionist models posit that speech perception is mediated by prelexical representations that strip away subphonetic variation, discarding acoustic information which is not relevant for making (possibly probabilistic) categorical distinctions (McClelland \& Elman, 1986; Norris, 1994; Norris et al., 2000; Norris \& McQueen, 2008). At the other extreme, episodic models posit that speech perception is a direct mapping of detailed acoustic traces to linguistic units (often lexical items), preserving all of the fine-grained acoustic informa- 
tion (Goldinger, 1998; Johnson, 1997a, 2006; Pierrehumbert, 2002). These two perspectives constitute dramatically different approaches to the lack of invariance, each of which is insightful but also falls short in different ways.

On the one hand, in the face of the lack of invariance abstractionist theories have historically relied on an explicit process of "talker normalization" where acoustic cues are normalized by pre-linguistic perceptual processes such that a single set of cue values or template can be used for each category, regardless of the talker. For instance, vowel formant frequencies, which vary systematically with talker gender (Hillenbrand et al., 1995; Peterson \& Barney, 1952) might be normalized with respect to the values of other formants, or the fundamental frequency (Strange, 1989). More recent approaches include what we termed "flat learner" models above, where a single set of cue-category mappings is dynamically adjusted based on recent input via something like Hebbian associative learning (Lancia \& Winter, 2013; Mirman et al., 2006).

On the other hand, episodic theories avoid the problem of talker normalization altogether, because the detailed acoustic traces of, for instance, individual word or vowel tokens are stored separately, and recognition proceeds on the basis of overall acoustic similarity. By storing large enough (e.g. wordsized) acoustic traces, each remembered episode encodes enough information to be useful in distinguishing individual talkers, supporting talker-specific cuecategory mapping (Goldinger, 1998; Johnson, 1997a; Pierrehumbert, 2002). For unfamiliar talkers, new episodes are recognized based on some measure of overall similarity to stored episodes from other talkers, allowing generalization across talkers.

\section{Insights and problems of each approach}

We can think of these as two extreme endpoints for how to map from acoustic cues to linguistic representations. Abstractionist accounts seek a minimal (sin- 
gle) set of cue-category mappings (possibly to be tuned and re-tuned based on experience), while episodic accounts use a maximal set of cue-category mappings, one for every observation ever made. Each approach is founded on useful insights. Abstractionist models are informationally efficient, collapsing all the variability across situations into a compact set of sublexical cue-category mappings which supports efficient generalization across lexical items. Episodic models are infinitely flexible, and can pick up on any informative set of cues simply by virtue of tracking all of them.

But each approach also comes at a cost. For abstractionist accounts, normalization is hard, if not impossible, given that much of the variability across talkers is not due to fixed (e.g. physiological) factors but rather stylistic and thus needs to be learned (Johnson, 2006; Pardo \& Remez, 2006). The Hebbian learning mechanisms that have been proposed typically operate at much longer time scales than the very rapid recalibration that is commonly observed (Guediche et al., 2014; Mirman et al., 2006). Even if the learning rate can be dynamically adjusted, existing proposals for "flat" learners cannot account for the speech perception system's sensitivity to structure (e.g. persistent talkerspecific representations). Episodic accounts which track raw acoustic episodes of a large enough size to simultaneously encode the phonetic features and the other acoustic information that tells you how to interpret those features (e.g. frication energy and adjacent vowel formants / $\mathrm{f} 0$ to get gender) cannot easily generalize recalibration to unheard words (Cutler et al., 2010). More importantly, episodic models cannot generalize across groups of different levels of specificity by simply recording acoustic episodes. Rather, they require some additional mechanism for "tagging" and filtering or weighting exemplars based on indexical variables. ${ }^{32}$

\footnotetext{
${ }^{32}$ Or, alternatively, a "smarter" similarity function that is somehow sensitive to the structure of the episodes, both linguistically and indexically (e.g., as discussed above, van den Bosch \& Daelemans, 2013).
} 


\section{Charting a middle course}

Of course, these criticisms are based on extreme or purist interpretations of these two approaches, and there is no reason why abstractionist models cannot be made to be a bit more like episodic models and vice-versa. Indeed, this has been the trend in recent years, and a number of hybrid models have been called for or proposed (e.g., Ernestus, 2014; Goldinger, 2007; McLennan, Luce, \& Charles-Luce, 2003). Of particular interest are proposals for abstractionist models that learn (Lancia \& Winter, 2013; Mirman et al., 2006), and episodic models that track episodes at sublexical granularity (Pierrehumbert, 2006) or tag episodes with explicit indexical variables and use these to bias later recognition (Johnson, 2006, 2013).

In fact, the perspective offered by the ideal adapter suggests that some balance between complete abstraction and complete lack of abstraction is optimal. The particular balance depends both on the current situation (which might require more or less flexibility and hence sensitivity to individual episodes) and on the listener's previous experience. A listener who has experienced input from a broad range of different generative models will have reason to believe that flexibility will be required in the future, and thus will be more likely to display it. A Listener who has experienced a narrower range or more consistent groupings has required less flexibility. The ideal adapter framework relates an individual listener's (potentially idiosyncratic) structured experience with different cue-category mappings to the kind of representations that will best serve the listener's needs, interpolating between complete abstraction and no abstraction. Like the rational model of categorization (Anderson, 1991), the ideal adapter framework proposes that listeners tune the specificity of their cue-category mappings based on their beliefs about the underlying structure of the world. 
The ideal adapter framework thus incorporates insights from both abstractionist and episodic approaches. On the one hand, like episodic approaches, it recognizes the need to estimate cue distributions. On the other hand, it recognizes that there is a substantial benefit from having only a compact set of generative model parameters that need to be adapted in each situation. In this sense, our approach is inspired by normalization accounts. However, instead of adjusting the acoustic cue space to make all talkers align with a fixed set of cueto-category mappings, the ideal adapter adjusts the category-to-cue mappings for each situation. We have also tentatively proposed that there is a parallel trade-off at a higher level, in estimating the distribution of generative models themselves across situations. This suggests that listeners might be able to use exemplars in generative model parameter space (rather than acoustic space) to estimate, in an approximate, boundedly-rational way, the overall distribution of generative models across situations (Ashby \& Alfonso-Reese, 1995; Gibson et al., 2013; Griffiths, Sanborn, Canini, \& Navarro, 2008) and to adapt to the current situation (Shi et al., 2010). However, there is also a benefit from abstracting away from individual situations by explicitly representing summaries of different group-level distributions in order to generalize across groups. While there is some evidence that phonetic adaptation reflects updating of top-down category-to-cue representations rather than bottom up cue warping (Dahan, Drucker, \& Scarborough, 2008), further work is required to determine whether this is what human listeners always do and to computationally flesh out these different approaches.

\section{The role of subphonetic detail}

The importance of subphonetic detail for categorizing speech has long been acknowledged, both by probabilistic, ideal observer models (Clayards et al., 2008; Feldman, Griffiths, \& Morgan, 2009; Norris \& McQueen, 2008; Sonderegger \& 
Yu, 2010) and others (McClelland \& Elman, 1986; Norris, 1994; Norris et al., 2000). For example, subphonetic detail is necessary for making linguistic inference in the face of variability and uncertainty. Higher levels of analysis benefit from knowing not just which phonetic category is most likely, but how certain that judgement is and what plausible alternatives are. To the extent that uncertainty can be carried through the different levels of inference, later evidence can be integrated rationally, allowing the listener to revise earlier interpretations when necessary. So-called right-context effects in spoken language processing show that listeners do, in fact, maintain uncertainty over the categorization of previous input (Bard et al., 1988; Connine et al., 1991; Dahan, 2010; Grosjean, 1985), and there is some evidence that the same occurs in written language processing, too (Levy, Bicknell, Slattery, \& Rayner, 2009). Conversely, people are sensitive to information from anticipatory coarticulation (Dahan, James S. Magnuson, Tanenhaus, \& Hogan, 2001; Marslen-Wilson \& Warren, 1994; McQueen, Norris, \& Cutler, 1999; Whalen, 1984), where upcoming segments change how an earlier segment is produced, providing probabilistic information about how to categorize later input and the underlying words.

The ideal adapter makes an even stronger claim about the importance of subphonetic detail. Compared to just speech recognition, a different (but overlapping) type of subphonetic detail is necessary in order to achieve robust categorization in the face of situational variability. We have argued that the distributions of cues corresponding to a category change across situations, and that robust categorization requires that these distributions be tracked. Such distributional learning relies on predictions about the subphonetic distribution of cues expected based on prior experience in order to detect when, and determine how, the current distributions deviate from what is expected. Two subphonetic variants of a particular category which would be classified-even probabilistically-in exactly the same way can nevertheless in principle pro- 
duce very different adaptation effects because they signal different underlying distributions. That is, in order to effectively update beliefs about the distribution of cues for each category, the speech perception system needs to be sensitive, at some level, to types of subphonetic differences that are irrelevant for classification.

Moreover, because different talkers can produce different distributions (which are distinguished by potentially subtle subphonetic differences), subphonetic detail provides information about who is talking (Creel \& Bregman, 2011; Pardo \& Remez, 2006). In a world where phonetic category distributions vary systematically as a function of who is talking, any information about the talker, even at a general level like gender or language background, is informative about what kind of category distributions will most likely be appropriate. This not only determines how adaptation proceeds but also can determine how speech sounds ought to be categorized. Much of this information is contained in subphonetic details like voice quality (Pardo \& Remez, 2006), but also, as we have argued, in subphonetic variation in phonetic cues themselves. Thus, our analysis suggests that even at fairly high levels of processing, the speech recognition system should be sensitive to subphonetic variation even when this variation is not directly informative about categorization.

\subsubsection{Implications of the ideal adapter framework beyond speech perception}

We now turn to the broader implications of this framework for speech perception, acquisition, and language comprehension. We begin by discussing the relation between adaptation in adults and language acquisition in infants. We then discuss how the proposed framework highlights an important parallel between learning (in the form of adaptation) and processing. Following that, we 
summarize parallels that the proposed account highlights between speech perception and language processing beyond speech perception. Finally, we suggest how our computational analysis of speech perception and the resulting models might be realized as process and mechanistic models, and some of the challenges in doing so.

\section{Parallels between acquisition and adaptation: Both can be understood as a form of distributional learning}

We have proposed that adaptation is a form of distributional learning and hence that distributional learning is a central part of processing spoken language by adult listeners in the face of variability across situations. Distributional learning has also been proposed as a mechanism by which linguistic categories are learned during language acquisition in infants (e.g., Aslin, Saffran, \& Newport, 1998; Gómez \& Gerken, 2000; Saffran, Aslin, \& Newport, 1996; Wonnacott, Newport, \& Tanenhaus, 2008) and adults (e.g., Pajak \& Levy, 2011; Saffran, Newport, \& Aslin, 1996). This includes the acquisition of phonetic categories (e.g., McMurray, Aslin, \& Toscano, 2009; Toscano \& McMurray, 2010; Vallabha et al., 2007). For example, infants have demonstrated sensitivity to the distribution of acoustic cues to phonetic categories as early as 6 months of age (Maye, Werker, \& Gerken, 2002). This parallel has been taken to suggest that language acquisition and adaptation can be attributed to the same implicit learning mechanisms, which continue to operate throughout life (e.g., Botvinick \& Plaut, 2004; Chang et al., 2006; Elman, 1990). We briefly review the arguments and challenges for such accounts.

While the adult listener has uncertainty about the distribution of cues associated with each category, the language learner faces the additional challenge of not knowing what the underlying categorical structure is. On top of the distributions (e.g. mean and variance), the language learner must therefore infer 
the existence and number of categories. Additionally, infant language learners must arguably do so from unlabeled data, so that at least the earliest stages of language acquisition require unsupervised learning. Given these prima facie differences between adaptation and acquisition, it is striking that distributional learning models similar to the one we propose here have been found to account for phonetic category acquisition data (Feldman, Griffiths, Goldwater, \& Morgan, 2013; McMurray, Aslin, \& Toscano, 2009; Vallabha et al., 2007), as well as acquisition of other linguistic structures (M. C. Frank, Goodman, \& Tenenbaum, 2009; O'Donnell, Snedeker, Tenenbaum, \& Goodman, 2011).

This and the work we present here have two implications for future work. First, our analysis of adaptation as central to speech processing suggests that the distributional learning mechanisms that seem to underly acquisition might continue to operate throughout life (as also proposed in Botvinick \& Plaut, 2004; Chang et al., 2006; Elman, 1990). Adult listeners have acquired rich distributional knowledge about both the cue-to-category mappings (generative model) of their native language and the distribution of these mappings across talkers. Still, even adult listeners are routinely exposed to situations in which they need to learn novel statistics. In ongoing work, we have found that a model which correctly infers that there are two voicing categories based on unlabeled distribution of VOTs can also account for adult listeners shifts in categorization after hearing unlabeled, shifted distributions of VOTs, and strikingly can do so using a single set of parameters (Toscano, C. M. Munson, Kleinschmidt, and Jaeger, under revision). Thus it appears that some distributional learning may be involved from acquisition to adult language use, echoing lifelong learning accounts.

In this context, it is worth noting that research on phonetic category acquisition has generally employed unsupervised learning models. The model we used in Part I was supervised. This is, however, not an in-principle limitation 
of the ideal adapter framework. Indeed, adults clearly can engage in unsupervised adaptation (Clayards et al., 2008; C. M. Munson, 2011). Further work is required to better understand the relative role of supervised and unsupervised learning (or the continuum between these extremes; e.g., Gibson et al., 2013; Zhu, Rogers, Qian, \& Kalish, 2007) both during acquisition and in speech perception in adults.

Second, as we have pointed out, the distributional statistics of phonetic categories depend on talkers, accents, etc. (e.g., Figure 2.1). This raises the question of how infants acquire categories statistically unless they separate the observations they make based on who is talking. This is an underexplored question that deserves further attention in future work. Preliminary evidence comes from White and Aslin (2011), who find that 20-month-old toddlers demonstrate phonetic recalibration in paradigms similar to those used in research on adults. This suggests that infants may be learning talker- or situation-specific phonetic category representations, as we have argued for adults. Furthermore, some computational studies demonstrating the ability of distributional learning algorithms to extract categorical structure have used input data that comes from multiple talkers, even collapsing across gender (e.g., Feldman, Griffiths, Goldwater, \& Morgan, 2013). This increases category overlap, which a priori would seem to make it more difficult to learn good phonetic categories. If infants separate tokens according to who produced them (or even according to nonphonetic features like voice quality) then at least some of this difficulty may be overstated.

However, tracking talker-specific distributions of cues also means that learners need to track a set of speech sound statistics for each individual talker, instead of just a single set of language-level distributions (as is typically modeled). This introduces two types of costs. First, it requires more statistics to be represented (remembered). Second, there are fewer observations available 
for each (talker-specific) generative model that needs to be learned, decreasing the reliability of these models. One way to ameliorate both costs is to recognize generalizations across talkers. In any case, however, the costs introduced by talker-specific or group-specific expectations can be justified when the variation between talkers is so large that the differences in the cue distributions of phonetic categories within each talker are obscured. Future work could employ computational simulations to probe the statistical conditions under which this trade-off is predicted to work out in favor for talker- and group-specific learning. Similarly, more experimental research is required on the changes in the way we learn and generalize throughout language acquisition.

\section{Parallels between processing and learning: Both involve inference}

Our ideal adapter framework is inspired by previous work which treats speech perception as a problem of inference under uncertainty (Clayards et al., 2008; Feldman, Griffiths, \& Morgan, 2009; Norris \& McQueen, 2008; Sonderegger $\& Y u, 2010)$. Like this previous work, we analyze the problem of speech perception via an ideal listener model where processing depends on the listener's model of the statistics of phonetic categories. However, the models developed in this previous work generally assume (tacitly) that for the purposes of adult language processing, these statistics are known and fixed. Our analysis points out that this is not a reasonable assumption, because the lack of invariance introduces variability in these statistics across situations.

In the proposed framework, the lack of invariance means that learning is closely intertwined with processing of spoken language. One insight of the ideal adapter is that processing and learning are both instances of inference under uncertainty. An ideal listener uses knowledge of how phonetic categories generate acoustic cues in order to determine which underlying category best explains an observed cue. In the same way, an ideal adapter uses knowledge 
of how different generative models produce different distributions of acoustic cues in order to update their beliefs about the appropriate generative model for the current situation. That is, in the ideal adapter framework, adaptation is inference at another level, inference of generative models.

There's a deeper parallel between processing and learning in speech perception, as well. Phonetic categories don't occur arbitrarily, in isolation, but have a regular structure determined by, for instance, the words of the language. An ideal listener uses knowledge about this structure to narrow down the possible explanations for a given acoustic cue through the prior $p(c)$ (Feldman, Myers, White, Griffiths, \& Morgan, 2013). In the same way, we have proposed that an ideal adapter should exploit the fact that changes in phonetic category distributions are systematically related to changes in talker, dialect, etc. This allows an ideal adapter to quickly and efficiently infer (re-learn) the distributions of a familiar talker when encountering them again, or to generalize from experience with talkers from a particular foreign accent group to a novel talker from the same group.

\section{Language understanding beyond speech perception}

The idea that language understanding involves prediction and inference under uncertainty has also guided work on language processing beyond speech perception. For example, expectation-based theories of sentence processing hold that comprehenders use knowledge of the statistics of syntactic structures in order to generate expectations about upcoming material (Hale, 2001; Levy, 2008b; M. C. MacDonald, Pearlmutter, \& Seidenberg, 1994; M. C. MacDonald, 2013; Tanenhaus, Spivey-Knowlton, Eberhard, \& Sedivy, 1995). As for speech perception, reliance on such statistics is efficient (Levy, 2008b; N. J. Smith \& Levy, 2013). As predicted by these theories, processing of structures which are highly likely given, say, a particular verb is faster than an alternative structure which 
is less likely (Garnsey, Pearlmutter, Myers, \& Lotocky, 1997; M. C. MacDonald et al., 1994; Staub \& Clifton, 2006; Trueswell \& Tanenhaus, 1994). Similarly, word-by-word processing times in reading have been found to increase with decreasing contextual predictability of the word (specifically, its contextual surprisal, Demberg \& Keller, 2008; Hale, 2001; S. L. Frank \& Bod, 2011)

As is the case for phonetic categories, these statistics vary across situations (see Fine, Jaeger, et al., 2013, for references). Thus, the same argument that we have made here for phonetic categorization-that effective comprehension relies on good estimates of the talker's generative model and deviations from expected statistics will lead to changes in comprehension-applies to syntactic processing, as well. Indeed, recent studies show that comprehenders do adapt to changes in the statistics of syntactic structures, with repeated exposure to a previously rare structure facilitating processing of that structure (Fine, Qian, Jaeger, \& Jacobs, 2010; Fine, Jaeger, et al., 2013; Jaeger \& Snider, 2013; Kaschak \& Glenberg, 2004).

These syntactic adaptation effects have much in common with phonetic adaptation. They are both rapid, occurring after only tens of exposures to the critical structure (Fine, Jaeger, et al., 2013; Kaschak \& Glenberg, 2004), and there is some evidence that they are persistent, lasting over multiple days (Wells, Christiansen, Race, Acheson, \& MacDonald, 2009), and that unusual syntactic preferences can be explained away (Hanulíková, van Alphen, van Goch, \& Weber, 2012). Consistent with the hypothesis that such adaptation generally serves to make language comprehension robust to systematic situational variability, Kamide (2012) found that comprehenders tracked talker-specific syntactic preferences (high- vs. low-attachment), and that this adaptation was reflected in online processing.

Further reinforcing the tentative parallels between syntactic and phonetic adaptation, belief updating models similar to the one presented here can quan- 
titatively account for these syntactic adaptation effects (Fine, Qian, et al., 2010; Kleinschmidt, Fine, \& Jaeger, 2012). Intriguingly, as we found here, these models also fit best with low effective prior sample sizes.

Similar findings are emerging for prosodic processing (Kurumada, Brown, Bibyk, Pontillo, \& Tanenhaus, 2013), phonotactic constraints (Chambers, Onishi, \& Fisher, 2010; Dell, Reed, Adams, \& Meyer, 2000; Goldrick, 2004; Warker \& Dell, 2006), pragmatic processing (Grodner \& Sedivy, 2011), and semantic interpretation, such as quantifier processing (Yildirim, Degen, Tanenhaus, \& Jaeger, 2013). This suggests that not only do people use their previous experience with the statistics of their language to predict upcoming material in order to facilitate comprehension, but that they are tracking situation-specific statistics and tuning their expectations to reflect changes in such statistics. The current work thus contributes to a growing literature that considers language comprehension to be intimately tied to adaptation and implicit learning (see also Dell \& Chang, 2014; Chang et al., 2006; Fine, Jaeger, et al., 2013; Jaeger, 2013; Jaeger \& Snider, 2013; M. C. MacDonald, 2013).

\section{Perception and learning beyond language}

The problem of achieving robust speech perception in many situations in the face of the lack of invariance is not unique to speech or language. Generally, agents (both people and other animals) need to act effectively based on sensory cues, and the mapping from cues to the appropriate actions (or interpretations, more generally) can vary quite a bit. There is a very long literature on how agents manage to act appropriately in a variety of situations. Much of this comes down to learning new or adjusted cue-outcome associations, and as such the majority of the classical behavioral paradigms expose animals to highly novel cue-outcome mappings, in order to better assess and understand learning. However, a recurring theme in this work is that learners have to func- 
tion in a multi-context world, where the cue-outcome mappings are not simply drawn from a single random distribution but rather depend systematically on factors of the underlying context.

Much of the work on such multi-context learning treats it as a changedetection problem, where the learner's strategy is to detect when the context has changed and then change their learning strategy by, for instance, increasing the learning rate (e.g., Courville et al., 2006; Gallistel et al., 2001). The ideal adapter framework offer an alternative to change detection models (see also related proposals discussed in Qian et al., 2012). By trying to infer the underlying generative model (or cue-outcome mapping) in each situation, changes in context can be detected implicitly. Further, by tracking context-specific generative models, previous experience can be used to efficiently re-learn previously encountered contexts.

There is indeed some evidence that learners do not abandon previously learned associations when learning new associations. For instance, extinction (unlearning) of a classically conditioned response (e.g. a fear response or eyeblink) is not absolute, and the original conditioned association is still present and can re-appear under a variety of circumstances from re-introduction of the original context, very brief exposure to the conditioned association, or simply spontaneously (Thanellou \& Green, 2011; Bouton \& King, 1983; Sissons \& R. R. Miller, 2009). Similarly, early exposure to perturbed audio-visual spatial correspondences in barn owls leads to more rapid re-adaptation to the same perturbation later in life, even with substantial un-perturbed experience in between (Körding, Tenenbaum, \& Shadmehr, 2007; Knudsen, 1998; Linkenhoker et al., 2005). Similarly, talker- and group-specific expectations in speech perception could be seen as resulting from context-sensitive associative learning, although we have not described it as such.

All of this raises the question as to whether the very same computational 
framework developed here-the ideal adapter-could be directly applied to the problem of domain-general learning in a multi-context world. It is not entirely clear that the entities of the ideal adapter can be mapped to more general settings, even if the central idea is applicable. What is, for instance, the analogue of a talker, or a phonetic category?

Speech perception as a computational problem is characterized by two, largely orthogonal, types of structure: linguistic and indexical. Linguistic structure refers to the fact that acoustic cues signal the presence of underlying phonetic categories, lexical items, syntactic structures, etc. Indexical structure refers to the fact that these cue-category mappings-which we have referred to as the generative model-vary across situations based (in large part) on who is talking, and other indexical variables like accent, dialect, gender, etc. The fact that the same set of generative model parameters (e.g. mean VOT of $/ \mathrm{p} /$ ) are relevant for all (or nearly all) talkers means that listeners have a lot to gain by combining experience with different generative models in different situations. But the same may not be true of other domains, where the underlying categorical structure of the stimuli itself varies across contexts.

This is not to say that the basic logic of the ideal adapter framework does not apply: people (and other agents) can benefit from tracking the structure of how cue statistics vary across situations to the extent that this variation is structured. By the same logic, a learner should not build a model of how sensory statistics vary across situations if that variability is unpredictable. In such a domain, the ideal adapter predicts that people would not show situation-specificity, but rather continuously adapt. ${ }^{33}$ Similarly, in a domain where the variability across situations is informative about both the cue-category mappings and what categories are relevant, then there is little benefit to tracking the distribution of

\footnotetext{
${ }^{33}$ This leaves aside the possibility of any higher-level inference about whether or not situation-specificity is appropriate that people may be doing.
} 
category statistics across situations. That is, if each category is only relevant in one particular situation, then by the logic of the ideal adapter framework, people might not generalize across situations.

This is also an argument for speech perception as a good test case for theories of multi-context learning. Variability in speech is highly structured-both linguistically and indexically—so that listeners pick up on and use both kinds of structure.

\subsubsection{Conclusion}

The proposed ideal adapter framework provides a potential solution to one of the oldest questions in research on speech perception: how do listeners overcome the computational problem caused by the lack of invariance of the speech signal? Recent proposals treat speech perception as a problem of inference under uncertainty about the phonetic category (or other linguistic unit) that a talker intended to produce (e.g., Clayards et al., 2008; Feldman, Griffiths, \& Morgan, 2009; Norris \& McQueen, 2008). The ideal adapter extends these models by treating speech perception as a problem of inference under uncertainty at multiple levels. Robust speech perception requires that listeners continuously draw inferences not only about what the talker is trying to communicate, but also about the cue-category mappings that the talker is using (i.e., the talker's generative model). Moreover, in order to determine what previous experience is relevant in making these inferences, and how relevant it is, these inferences in turn depend on higher-level inferences about who the talker is. This ranges from specific talker identity-supporting recognition of familiar talkers—-to more general groups like gender, accent, or dialect—supporting generalization across similar talkers. The proposed multi-level inference solution can capture a variety of otherwise puzzling properties of speech adapta- 
tion and provides a guiding framework for future research on speech perception, adaptation, and generalization.

The challenges posed by variability are not unique to speech perception, but rather general to the problem of effective perception and action in a variable world. This problem has been explored in the context of motor control (e.g., Körding, Beierholm, et al., 2007) and reinforcement learning (e.g., Cho et al., 2002; Gallistel et al., 2001), where it is typically cast as a problem of detecting changes in the statistics of the local environment (change detection). Our proposal highlights the fact that in a world where substantial parts of crosssituation variability are not random, but rather structured, simply detecting changes is not enough. Rather, learners can benefit from inferring the underlying structure to cross-situation variation, in order to recognize familiar situations and generalize to similar situations. In speech perception, the major source of variation across situations is the talker, but the same logic can be applied to other domains (Qian et al., 2012, 2015). The ideal adapter highlights the potential of speech perception to serve as a model organism for understanding perception in a variable-but structured-world, and suggests that superficially unrelated phenomena from non-linguistic perceptual/motor domains might be informative about language processing and acquisition and vice-versa. 


\section{Re-examining selective}

\section{adaptation: Fatiguing feature}

\section{detectors, or distributional}

\section{learning? ${ }^{1}$}

Repeated exposure to a prototypical example of a phonological category seems to 'shrink' that category. For example, exposure to a reasonably good example of a / b/ many times in a row makes listeners less likely to classify other sounds on a /b/-/d/ continuum as /b/ (Eimas \& Corbit, 1973). The discovery of this effect, known as selective adaptation, led to a great deal of excitement among speech perception researchers. By analogy with similar adaptation effects in visual psychophysics, it promised a "psychophysicist's microelectrode" (Frisby, 1979; Mollon, 1974), a powerful and general method of probing the neural code by which continuous acoustic input is transformed into discrete linguistic units, and hence potentially resolving fundamental debates about the psychological reality and properties of phonemes and phonetic features hypothesized by formal linguistic analysis. Selective adaptation effects in visual perception, for

\footnotetext{
${ }^{1}$ The contents of this chapter were previously published as Kleinschmidt, D. F. \& Jaeger, T. F. (2016a). Re-examining selective adaptation: Fatiguing feature detectors, or distributional learning? Psychonomic Bulletin \& Review, 23(3), 678-691. doi:10.3758/s13423-015-0943-z
} 
instance, were critical in establishing - based on behavior alone-the features used by the visual system to analyze visual input (e.g., Blakemore \& Campbell, 1969), and it was hoped that the same sort of effects in speech perception would be able to establish the existence of feature detectors for abstract linguistic categories like phonemes and phonetic features like voicing. But this excitement was quickly tempered for a variety of reasons. Chief among them were findings suggesting that selective adaptation reflects something more like fatigue of general sensory feature detectors, rather than language-specific processing of speech sounds (e.g., Remez, 1979; Samuel \& Newport, 1979; Schouten, 1980; Roberts \& Summerfield, 1981). More deeply problematic, the patterns of adaptation observed were not consistent with fatigue of the kind of feature detectors that had been assumed (see Remez, 1987, for an excellent critique). It became clear that adaptation effects needed to be explained as phenomena in their own right, and subsequently selective adaptation fell out of favor in work on speech perception (cf. Remez, 1987; Samuel, 1986).

In the decades since the rapid rise and fall of selective adaptation, we have learned a great deal about adaptive properties of sensory systems generally, and speech perception in particular. We argue that taken together, these bodies of work suggest that it is time to re-examine the phenomenon selective adaptation. Two developments contribute to our argument. First, recent work challenges the idea that sensory adaptation is best thought of as the fatigue of feedforward feature detectors, or more broadly as a mechanistic property of sensory systems. Rather, an emerging perspective views adaptation as a computational property of sensory systems, where the sensitivity of the sensory system (with its limited neural resources) is re-aligned to information that is relevant to the task at hand and meaningful in the current environment (e.g., Gutnisky \& Dragoi, 2008; Kohn, 2007; Stocker \& Simoncelli, 2006; Webster et al., 2005). While in some cases this re-alignment might be achieved by detector fatigue, 
there is a growing body of work that cannot easily be accounted for in this way (which we will discuss below). Second, the last decade of speech perception research has revealed that, far from a series of fixed feature detectors, the speech perception system is adaptive and flexible in smart ways (e.g., Bertelson et al., 2003; Bradlow \& Bent, 2008; Clayards et al., 2008; Kraljic, Samuel, \& Brennan, 2008; Maye, Aslin, \& Tanenhaus, 2008; Norris et al., 2003). Plasticity and context-sensitivity are increasingly understood to be central features of a speech perception system that has to function in a world where the cueto-category mapping depends on aspects of the local environment, like who is talking (Norris et al., 2003; J. Huang \& Holt, 2012; Laing, Liu, Lotto, \& Holt, 2012).

These developments, we argue, undermine two of the basic assumptions of early work on phonetic selective adaptation: that speech perception is (1) performed by acoustic-phonetic feature detectors that (2) become fatigued if they are stimulated too often. In this paper, we focus on the fatigue assumption (see Remez, 1987, for a rejection of the feature detector assumption). In part, we focus on fatigue because contemporary speech perception research views selective adaptation as a fatigue effect that is qualitatively different from other sorts of plasticity (e.g., Grabski, Tremblay, Gracco, Girin, \& Sato, 2013; Vroomen, van Linden, Keetels, et al., 2004; Vroomen, van Linden, de Gelder, \& Bertelson, 2007; van der Zande, Jesse, \& Cutler, 2014; Zäske, Fritz, \& Schweinberger, 2013). More importantly, we focus on fatigue here because the developments mentioned above do more than just undermine the fatigue assumption. Additionally, we propose, they provide a productive lens through which to reexamine the phenomenon of selective adaptation, and speech perception more generally.

A common thread that runs through recent work on sensory adaptation and flexibility in language comprehension is the idea of distributional learning 
across multiple levels. The sensory information that is relevant or meaningful in the current environment depends on the distribution of sensory features in that environment. Sensory systems adapt to these properties, and to that extent they can be thought of as implicitly learning those distributions (Brenner et al., 2000; Fairhall et al., 2001; Gutnisky \& Dragoi, 2008; Sharpee et al., 2006; Stocker \& Simoncelli, 2006). At the same time, building on recent work that treats speech perception as statistical inference (e.g., Clayards et al., 2008; Feldman, Griffiths, \& Morgan, 2009; Kleinschmidt \& Jaeger, 2015; Norris \& McQueen, 2008; Sonderegger \& Yu, 2010), much of the flexibility of speech perceptionand language comprehension more broadly - can be seen as a form of distributional learning. In this view, listeners deal with talker-to-talker variability by updating their beliefs about the distributions of cues (acoustic, phonetic, lexical, etc.) that result when a talker produces a linguistic structure (phonetic category, word, syntactic structure, pragmatic intention, etc.; Fine, Jaeger, et al., 2013; Guediche et al., 2014; Holt, 2006; Idemaru \& Holt, 2011; Kleinschmidt \& Jaeger, 2015; Yildirim, Degen, Tanenhaus, \& Jaeger, 2016).

First, we briefly review the evidence that sensory adaptation in general is better viewed as a form of distributional learning, rather than the fatigue of static feature detectors. Second, we lay out a tentative proposal for how the logic of distributional learning applies to phonetic selective adaptation. Third, we show that much of the literature on selective adaptation which appears to contradict our proposal is actually predicted by it, given a modern understanding of distributional learning that occurs at multiple levels of representation. Fourth, and finally, we lay out what we see as some of the most pressing questions that this proposal raises for future work.

Our goal is not to show conclusively that selective adaptation is, in all cases, distributional learning. Rather, we hope that distributional learning provides a productive perspective for re-examining previous evidence, and renews inter- 
est in selective adaptation as an important phenomenon in need of explanation in its own right, rather than simply a methodological tool.

\subsection{Sensory adaptation as distributional learning}

Non-linguistic sensory adaptation (which usually means visual adaptation) has much in common with phonetic selective adaptation: repeated exposure to a stimulus attribute will reduce responsiveness to that attribute, both at the level of behavior (psychophysics) and neural responses (electrophysiology). We refer to this decrease in responsiveness as a negative after-effect. For example, after extended viewing of a vertical grating, the contrast threshold for detecting another vertical grating goes up, and the firing rate of neurons in the visual cortex which are sensitive to vertical orientation is reduced when viewing another vertical grating (cf. Kohn, 2007). These findings were originally explained as fatigue of neuronal populations which are primarily sensitive to the adapted feature (Blakemore \& Campbell, 1969), and when analogous negative after-effects of selective adaptation with speech were first discovered (Eimas \& Corbit, 1973), they too were chalked up to a similar mechanism (although not without debate, some of which we revisit below; e.g., Cole, Cooper, Singer, \& Allard, 1975; Ainsworth, 1977).

However, work in the decades since challenges the idea that adaptation is broadly the result of "dumb" fatigue of feature detectors (Kohn, 2007; Webster et al., 2005). We now know that adaptation does not just reduce the firing of neurons overall but actually changes their tuning, such that some stimuli might even elicit higher firing rates after adaptation (Dragoi, Sharma, \& Sur, 2000; Gutfreund, 2012; Kohn \& Movshon, 2004). Moreover, these changes in tuning have been shown to be coordinated across populations of neurons, and often in ways that increase the amount of information that is transmitted about stimuli 
in the local environment (Brenner et al., 2000; Fairhall et al., 2001; Gutnisky \& Dragoi, 2008; Sharpee et al., 2006). Specifically, changes in tuning seem to serve an increase in the perceptual resolution for the range and distribution of stimuli observed in the current environment (e.g., in an experiment).

This and related findings suggest that selective adaptation has to be understood at least in part as a computational property of sensory systems that need to use limited neural resources to efficiently represent information across a variety of environments (Brenner et al., 2000; Fairhall et al., 2001; Gutnisky \& Dragoi, 2008; Kohn, 2007; Sharpee et al., 2006; Wainwright, 1999; Webster et al., 2005). In order to allocate processing resources, sensory systems need to know, at a basic level, how the features they represent are statistically distributed in the current environment. The sensory information that is most likely and relevant changes from one environment to the next, and so sensory systems need to adapt to these changes in order to make the best use of their limited neural resources. One way to distinguish more stimuli using a fixed number of neurons is to change the stimulus features encoded by each neuron's signal based on the current environment, because the environment itself provides some clues as to which stimuli are most likely and how they ought to be interpreted. Viewing adaptation purely as a mechanistic property like feature detector fatigue misses this important point.

How does this computational perspective help to understand the effects observed in a typical adaptation experiment? Consider the extreme deviation such an experiment presents from everyday perception. The perceptual environments we encounter outside the lab tend to exhibit a lot of variability in stimulus features. In a typical adaptation experiment, however, the exact same stimulus is presented over and over again. This produces a highly concentrated, non-variable distribution. The typical behavioral and neural after-effects of adaptation can be attributed to the retuning of sensory systems to this new 
distribution, by, for instance, reducing noise or increasing selectivity to make finer-grained distinctions in the more narrow range of stimuli observed in the experiment (Gardner, Tokiyama, \& Lisberger, 2004; Stocker \& Simoncelli, 2006; Wainwright, 1999), or reducing sensitivity to highly predictable stimuli (Friston, 2005; Rao \& Ballard, 1999). In order to re-tune itself based on the distribution of sensory features, the sensory system has to, at least implicitly, learn those distributions. In this sense, selective adaptation can be thought of as distributional learning.

Research on selective adaptation in the visual domain provides two pieces of evidence in support of this view that we discuss next. Both of these phenomena reveal that it is critical to consider distributional learning at multiple levels: short-, medium-, and long-term distributions in the first case, and different levels of features (part vs. whole) in the second.

First, to the extent that adaptation is a consequence of distributional learning, stronger adaptation is predicted to occur when the current distribution deviates more from the expected distribution, and more learning is required. Chopin and Mamassian (2012) showed that, as predicted by distributional learning, in an (experimental) visual world where right-leaning edges are more common overall, seeing a series a right-leaning bars produces less of a negative after-effect, compared to a world where left-leaning edges are more common. Subjects were asked to say whether a bar was leaning to the left or the right, in a sequence of trials that contained vertical, left-leaning, and right-leaning bars. Critically, Chopin and Mamassian (2012) manipulated the short-term and long-term frequency of left- vs. right-leaning trials before each of the vertical test trials. When there were more right-leaning bars in the short-term history (last 3 minutes), subjects showed the typical negative after-effect, and were more likely to say that a vertical bar was leaning left. But when there were more right-leaning bars in the long-term history (from 2 to 10 minutes 
in the past), subjects showed a positive after-effect, and were biased to say a vertical bar was right-leaning. This effect runs contrary to the basic prediction of the neural fatigue theory of adaptation: more exposure to right-leaning stimuli should always result in fewer right-leaning responses, regardless of the long-term statistics of the current environment.
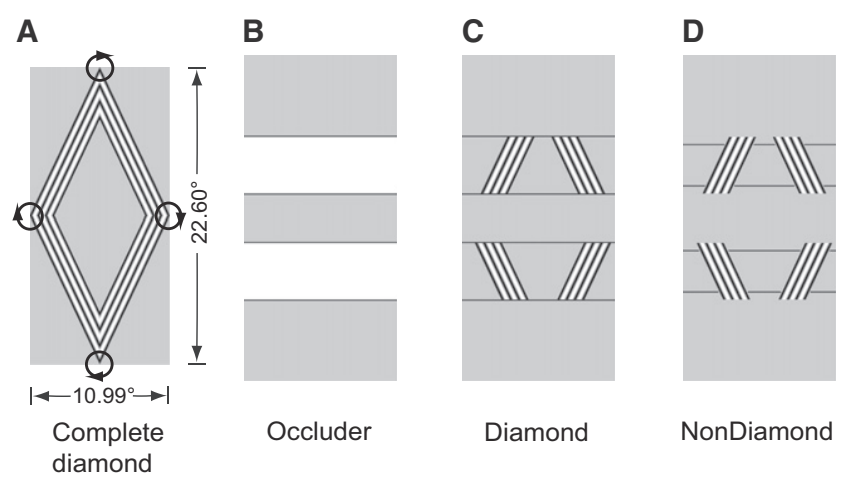

Diamond

NonDiamond
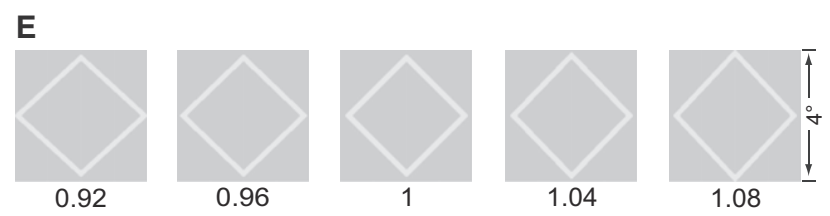

F

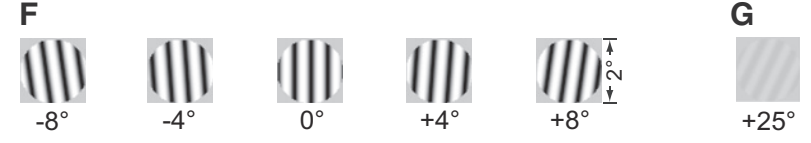

Figure 3.1: Adaptor and test stimuli used by He, Kersten, and Fang (2012). The adaptor stimulus was a diamond shape (A) that rotated behind three occluder bars (B). In the high-level condition (C), the lines indicating the edges of the occluders matched the portion actually occluded, leading to a percept of a moving diamond shape. In the low-level condition (D), the lines were moved, leading to a percept of four disconnected edges. The shape after-effect was tested by means of a tall-versus-wide diamond task (E), while adaptation to the edges themselves was tested via a tilt after-effect, measured with a leftversus-right leaning bar task $(\mathrm{F})$. Adaptation to the whole shape $(\mathrm{C})$ produced strong shape after-effects (E) but much weaker tilt after-effects (F) than the nonfused edges (D). Reprinted from Current Biology, 22 (11), He et al., "Opposite modulation of high- and low-level visual aftereffects by perceptual grouping" p. 1041, Copyright 2012, with permission from Elsevier.

Second, high-level features can change the expected distribution of lower- 
level features, and distributional learning predicts that such a change should lead to more or less adaptation to those lower-level features. For instance, the presence of a diamond shape predicts the presence of oriented bars corresponding to its edges. Sensory adaptation indeed appears to be sensitive to this dependence as well: adaptation to a higher-level feature-like a shapereduces or eliminates the after-effects produced by its component featureslike individual edges - when presented in isolation (He, Kersten, \& Fang, 2012). By manipulating perceptual grouping of individual oriented bars, He et al. (2012) changed whether they were perceived as edges of a single figure (a diamond, Figure 3.1, C) or as separate objects (four bars, Figure 3.1, D). When the bars were perceived as separate, each feature individually produced the typical after-effect. However, when all of the features were perceptually grouped into a diamond, the after-effects of the individual features were substantially reduced. Instead, a higher-level shape after-effect was obtained. Again, this effect runs contrary to a fatigue theory of adaptation. Subjects were exposed to exactly the same low-level features in both cases (edges with the same orientation). Thus the corresponding edge detectors should be subject to the same fatigue. But only when there is no higher-level feature that explains the oriented edges is there any adaptation to the oriented edges themselves.

In both of these cases, attributing selective adaptation to fatigue fails to explain the observed effects. Distributional learning, however, offers a possible explanation. In a distributional learning theory of adaptation, negative aftereffects happen because the sensory system has adjusted to an unusual distribution of stimuli. The degree of adjustment, and hence the strength of the negative after-effects, is determined by just how unusual that stimulus distribution is, compared to what is typical or expected. Critically, a stimulus distribution that would be highly unusual on its own might be perfectly typical if it is similar to the distributions encountered in medium-to-long-term history 
(e.g., as in Chopin \& Mamassian, 2012) or predictable given the presence of higher-level features (e.g., as in He et al., 2012). To a first approximation, a distributional learning theory thus correctly predicts that such predictable or expected deviations in sensory statistics should produce weaker after-effects than unpredictable deviations. ${ }^{2}$

A growing body of evidence thus suggests that non-linguistic sensory adaptation is intimately tied to the distribution of sensory features in the current environment. Rather than inducing the same adaptation regardless of the environment, the effect of repeated exposure to low-level features depends on higher-level statistics of the environment. This work shows that the distribution of sensory features in isolation are not enough for understanding adaptation. Rather, it is necessary to consider sensory feature distributions across multiple levels, both in the sense of part-whole feature relationships (He et al., 2012) and in the sense of short-, medium-, and long-run distributions of a single feature (Chopin \& Mamassian, 2012). Next, we review how the same distributional learning logic can be applied to selective adaptation in speech perception.

\subsection{Phonetic selective adaptation as distributional learning}

At the most basic level, the distribution of acoustic cues determines the mapping from acoustic cues to the underlying phonetic categories. Moreover, these distributions can vary quite a bit from one talker to the next. This variability means that in order to robustly recognize phonetic categories, the speech

\footnotetext{
${ }^{2}$ An important caveat is that if the statistics deviate too much from what is expected, then learning may not be possible at all. One notable example from speech perception is that listeners do not adapt fully to highly unusual accents (e.g., Idemaru \& Holt, 2011; Sumner, 2011).
} 
perception system must, in some way, be sensitive to changes in these statistics (Kleinschmidt \& Jaeger, 2015; McMurray \& Jongman, 2011). One way of achieving this sensitivity is by distributional learning (for a review of additional means, such as normalization, see Weatherholtz \& Jaeger, 2016). Indeed, research on speech perception has accumulated an impressive body of evidence that adult listeners do engage in distributional learning during speech perception, both at the level of cues (e.g., recalibration/perceptual learning, Bertelson et al., 2003; Clayards et al., 2008; Feldman, Myers, et al., 2013; Kraljic \& Samuel, 2007; C. M. Munson, 2011; Norris et al., 2003) and at the level of phonetic categories themselves (e.g. learning novel phonotactic constraints, Dell \& Warker, 2004; Warker \& Dell, 2006; Warker, Xu, Dell, \& Fisher, 2009). Recent work further suggests that adults also engage in distributional learning at higher levels of linguistic representation, like syntactic structures (Chang et al., 2006; Fine, Jaeger, et al., 2013; Jaeger \& Snider, 2013; Kamide, 2012) and pragmatics (Kurumada et al., 2014; Kurumada, Brown, \& Tanenhaus, 2012; Yildirim et al., 2016). As with general sensory adaptation reviewed above, this learning seems to be a form of "smart" plasticity that increases the speed and accuracy of language processing in the face of uncertainty and variability across environments (for review, see Kleinschmidt \& Jaeger, 2015).

Distributional learning in speech perception is a prerequisite for a distributional learning account of selective adaptation, but how does the logic of distributional learning play out for selective adaptation to phonetic input? Intuitively, the logic is the same as for adaptation in the visual domain, discussed above. Consider a typical phonetic adaptation experiment, where a listener hears a prototypical /b/ repeated over and over again. This leads to a very odd distribution of acoustic cues: typically, there is some variability in the signal, even when the same category is repeated by the same talker in the same phonological context (Allen, J. L. Miller, \& DeSteno, 2003; Newman et al., 2001). Such 

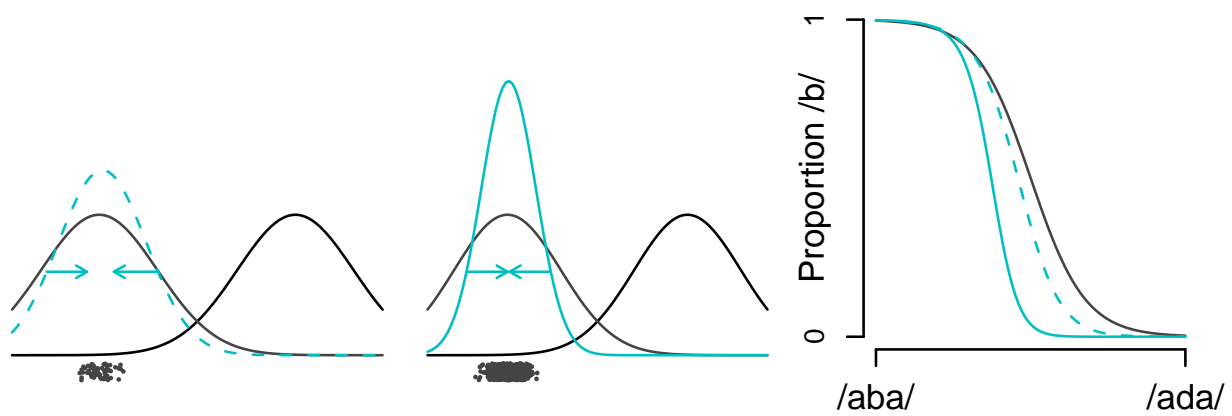

Figure 3.2: Distributional learning accounts for the negative after-effects observed in phonetic selective adaptation. Repeated exposure to a prototypi$\mathrm{cal} / \mathrm{b} /$ results in a distribution with much lower variance than normal (left). Learning this distribution means that previously ambiguous stimuli are now, to the listener, more likely to be occur if the intended category was /d/, resulting in a negative shift in the $/ \mathrm{b} /-/ \mathrm{d} /$ category boundary (right). Incremental belief updating predicts that the more exposure to this distribution the listener has, the more their beliefs should reflect it (left vs. middle) and the stronger the after-effect (right, dashed vs. solid).

a highly concentrated distribution might, intuitively, correspond to a talker who is very precise in how they produce their $/ \mathrm{b} / \mathrm{s}$. Such a talker is less likely to produce a / b/ with a cue value that, for a normal talker, would be ambiguous between a /b/ and a /d/. Consequently, if the listener has learned this changed distribution, then we would predict that they classify fewer items by the same talker on a /b/-to-/d/ continuum as /b/ (Figure 3.2).

This reduction in /b/ responses after / b/ exposure is exactly the negative after-effect (shift in category boundary) that characterizes selective adaptation in speech (Eimas \& Corbit, 1973; Samuel, 1986). Thus, qualitatively, distributional learning provides an account for selective adaptation, at least as far as its effects on classification are concerned. Quantitatively, too, distributional learning provides a good fit against data from selective adaptation: the incremental effects of repeated exposure to the same stimulus are well-described by a distribution learning model $\left(r^{2}=0.85\right.$, Kleinschmidt \& Jaeger, 2015).

The "ideal adapter" distributional learning model developed in Klein- 
schmidt and Jaeger (2015) predicts that listeners do not just track the variance of cue distributions, but the whole distribution, which includes at least both the means and variances of cue distributions. The same model can be applied to phonetic recalibration/perceptual learning, where listeners hear an atypical production of /b/ which is acoustically ambiguous between /b/ and /d/, but which is disambiguated by, e.g., a visual cue or lexical context. The resulting cue distribution is unusual both in terms of its mean and its variance. Recalibration is characterized by a positive after-effect: after such exposure, listeners classify more items from a /b/-/d/ continuum as /b/. Recalibration, unlike selective adaptation, has traditionally been described as a form of implicit learning (Norris et al., 2003; Vroomen, van Linden, Keetels, et al., 2004; Vroomen, van Linden, de Gelder, \& Bertelson, 2007). Nevertheless, the very same distributional learning model fits listeners' classification behavior in a recalibration experiment just as well as for selective adaptation $\left(r^{2}=0.86\right.$, Kleinschmidt \& Jaeger, 2015), using an identical set of parameters for both recalibration and selective adaptation. According to the ideal adapter model, the positive after-effect elicited by recalibration is typically due to the unusual mean of the cue values that listeners hear. By learning that the experimental talker produces their /b/ with a mean cue value that is more like /d/, listeners can infer that /d/-like cue values are more likely to be produced by this talker when they are saying /b/, resulting in a positive after-effect.

The ideal adapter makes a further, less intuitive prediction that has only recently been recognized. The distribution of cues in a typical recalibration experiment does not only have an unexpected mean; it also has unexpectedly low variance. This later property is shared with selective adaptation experiments. Distributional learning predicts that longer-term exposure to a distribution with lower-than-expected variance should produce a negative aftereffect. That means that the positive after-effect that is observed in perceptual 
recalibration should eventually be undone or perhaps even reversed. Quantitative simulations of an ideal adapter corroborate this intuitive prediction: with enough exposure to the same stimuli, the positive after-effects of recalibration can be canceled out, or even reversed (Kleinschmidt \& Jaeger, 2015, pp. 164-6). Moreover, this is exactly what Vroomen, van Linden, de Gelder, and Bertelson (2007) found for human listeners: exposing listeners to 256 repetitions of an ambiguous / $\mathrm{b} /$ lead to initial positive after-effects that were later canceled out. In recent work, we have replicated this effect (Kleinschmidt \& Jaeger, 2012). In the same experiments, we extended exposure to cue distributions with a range of means, from fully prototypical (as in selective adaptation) to fully ambiguous (as in recalibration) and a number of steps in between. As predicted by a distributional learning account, the observed after-effects formed a continuum: initially, exposure to cue distributions with shifted means lead to positive after-effects; with increasing exposure, however, these effects were overcome, undoing the positive after-effects and eventually even reversing them into negative after-effects. An ideal adapter model fits all of these exposure distributions with a single set of parameters, suggesting that distributional learning can account for the whole range of exposure conditions, including selective adaptation (Kleinschmidt \& Jaeger, 2015).

Finally, we note that the ideal adapter model is only one example of a broad class of models that, implicitly or explicitly, incorporate distributional learning. For instance, episodic and exemplar models (e.g., Goldinger, 1998; Johnson, 1997a) can be seen as implementing a form of implicit distributional learning (as kernel density estimation, cf. Sanborn et al., 2010; or importance sampling Shi et al., 2010). Distributional models of phonetic category acquisition (e.g., Feldman, Griffiths, Goldwater, \& Morgan, 2013; McMurray, Aslin, \& Toscano, 2009; Vallabha et al., 2007), if extended to adult phonetic adaptation data, would make similar predictions, possibly even using the same learning 
rates (Toscano, C. M. Munson, et al., submitted). Finally, other models that treat speech perception as a process of inference under uncertainty implicitly assume sensitivity to the underlying distributions (Clayards et al., 2008; Feldman, Griffiths, \& Morgan, 2009; Norris \& McQueen, 2008; Sonderegger \& $\mathrm{Yu}, 2010)$, even though they do not explicitly include distributional learning per se. While members of this broad class of models predict sensitivity to cue distributions, only the ideal adapter has been explicitly applied to selective adaptation data (Kleinschmidt \& Jaeger, 2015). It thus remains to be seen how well this class of models (rather than the ideal adapter model itself) might account for selective adaptation.

\subsection{Challenges to this view}

In summary, evidence from both speech perception and sensory adaptation in non-linguistic domains suggests that selective adaptation is better understood as distributional learning by sensory systems that are constantly adapting to changes in the statistical properties of the sensory world. However, the literature on phonetic selective adaptation presents a number of immediate challenges to this view. In this section we review some of the most striking, and argue that in large part they are actually predicted by a distributional learning account.

\subsubsection{Similar accounts, previously rejected}

Several previous proposals have treated selective adaptation in ways that bear resemblance to the current proposal. These accounts are widely considered to be rejected by existing evidence. As we lay out below, these previous accounts differ from the current proposal in critical ways. We argue that, in fact, the 
evidence that rejects them is entirely consistent with the sort of distributional learning we are proposing.

\section{Re-tuning, or changing category mean}

Ainsworth (1977) investigated whether selective adaptation could be due to adaption to changes in category means, a hypothesis he referred to as "retuning" of feature detectors. Ainsworth rejected this account because adaptation with sounds that are closer to the category boundary still produces a negative after-effect (Ainsworth, 1977). As Ainsworth pointed out, such changes cannot be accounted for by changes to only category means, which would produce a positive after-effect as in recalibration discussed above.

However, unlike Ainsworth's (1977) proposal, the ideal adapter account we described in the previous section assumes that listeners implicitly learn not only the category means, but rather the whole distribution (or at least both the mean and variance). As discussed in the previous section, such an ideal adapter account can capture the negative after-effect elicited by a typical selective adaptation paradigm, both qualitatively and quantitatively (Kleinschmidt \& Jaeger, 2015). More specifically, it predicts that long-term exposure to the same stimulus will, in many cases, elicit a negative after-effect, even when the initial after-effect is positive. This is precisely the pattern that is observed in experiments where listeners are tested at different stages of adaptation (Kleinschmidt \& Jaeger, 2015; see also Vroomen, van Linden, de Gelder, \& Bertelson, 2007; and the re-analysis of Samuel, 2001, therein).

These findings suggest an alternative explanation for the findings reported by Ainsworth (1977). As is typical for selective adaptation experiments, Ainsworth employed a paradigm in a large number of adapting trials preceded each test trial. It is thus likely that Ainsworth effectively tested long-term ef- 


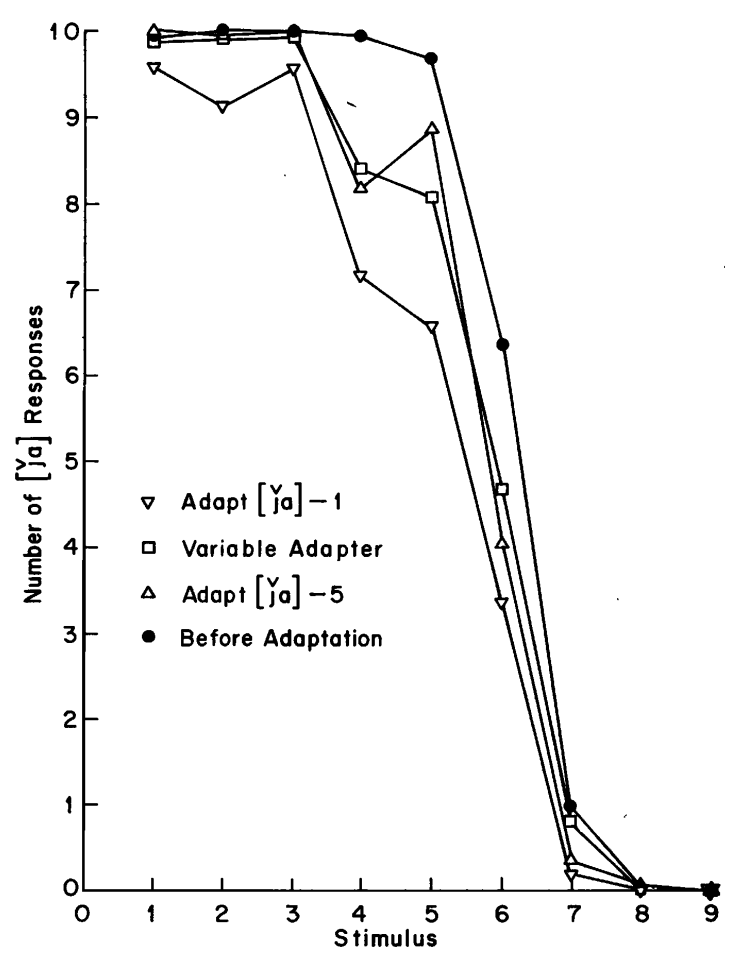

Figure 3.3: Selective adaptation data from Cole and Cooper (1977). The $x$-axis shows the test stimuli on a 1 to $9, / \mathrm{d}_{3} /$ (the " $\mathrm{j}$ " sound in jump) to /d/ continuum, and the $y$ axis shows subjects' average number of $/ \mathrm{d}_{3} /$ responses out of 10 repetitions of each test stimulus. The "variable adapter" condition was exposure to a uniform distribution of stimuli, from $x=1$ to 6 . Reprinted with permission from Cole and Cooper (1977). "Properties of friction analyzers for [j]". The Journal of the Acoustical Society of America, 62(1), 177. Copyright 1977, Acoustic Society of America.

fects of adaptation, in which case the negative after-effects Ainsworth observed are exactly what the ideal adapter distributional learning account predicts.

\section{Narrowing of selectivity, or changing category variance}

In a similar vein, Cole and Cooper (1977) investigated another type of distributional learning account, essentially assuming that listeners adapt only to changes in variance. Cole and Cooper referred to this as a "narrowing of selectivity". Based on data from several perception experiments, shown in Fig- 
ure 3.3, Cole and Cooper concluded that narrowing of selectivity alone is not sufficient to explain selective adaptation. If selective adaptation was caused by only adaptation to changes in category variance, than exposure to a range of adaptor stimuli with normal levels of variance should produce no selective adaptation at all. However, Cole and Cooper (1977) found that their "variable" adaptation condition (Figure 3.3, squares) produced a negative after-effect just as large as a non-variable condition with a single, intermediate adaptor (Figure 3.3, upward pointing triangles).

While this result cannot be explained in terms of adaptation to changes in variance alone, it is compatible with an ideal adapter. Recall that the ideal adapter predicts that listeners are sensitive to the whole distribution of cues (including the mean), not just the variance alone. All else being equal, distributional learning predicts that a high-variance adaptor distribution will produce a smaller negative after-effect than a low-variance distribution, but all else is not equal in Cole and Cooper (1977). The intermediate $/ \mathrm{d}_{3} /$ adaptor $(x=5)$ was substantially farther from the $/ \mathrm{d}_{3} /$ endpoint $(x=1)$ than the average of the variable- $/ \mathrm{d} 3 /$ condition $(\bar{x}=3.5$, a difference of 2.5 from the endpoint, vs. a difference of 4 for the intermediate adaptor). ${ }^{3}$ An adaptor closer to the category prototype is, in a distributional learning model, predicted to produce a stronger negative after-effect. This would mitigate the effect of the higher variance in the range condition, which could explain why the intermediate and range conditions produce comparable effects. In fact, the ideal adapter model qualitatively predicts that the low-variance, intermediate adaptor should lead to a slightly steeper category boundary, which is in fact what Cole and Cooper (1977) appear to have found (see Figure 3.3, $x=5,6,7$ ).

\footnotetext{
${ }^{3}$ The same argument holds when using frication duration-the actual physical dimension manipulated to construct the continuum-instead of continuum step, with one caveat: due to an error in constructing the stimuli (Cole \& Cooper, 1977, Footnote 1), steps $x=4$ and $x=5$ both had frication durations of $32 \mathrm{~ms}$, which means that the variability of the range condition is actually even lower than intended.
} 


\subsubsection{Selective adaptation to audiovisual adaptors}

One prediction of a distributional learning theory of selective adaptation is that adaptation should depend on the joint distribution of all relevant cues, including visual speech cues. However, studies using audiovisual adaptors (Roberts \& Summerfield, 1981; Saldaña \& Rosenblum, 1994) have been argued to support the view that selective adaptation is due to the fatigue of specifically auditory feature detectors. These audio-visual adaptors had large, categorical mismatches, which are intended to produce a McGurk Effect percept, where the perceived category of the audiovisual stimulus is different than the auditory component alone (McGurk \& J. MacDonald, 1976). Saldaña and Rosenblum (1994) used an audio-/ba/, visual-/va/ adaptor stimulus which was consistently identified as /va/ by participants. This adaptor produced an after-effect on a /ba/-/va/ continuum the same size as when the audio / ba/ component was presented separately. Saldaña and Rosenblum (1994) interpreted this aftereffect as evidence that listeners adapted to the audio component alone, rather than the integrated percept of /va/. ${ }^{4}$ As a result, Saldaña and Rosenblum (1994) rejected explanations of selective adaptation as learning at the level of phonetic categories-as in distributional learning - in favor of a process of purely lowlevel acoustic feature detector fatigue.

However, it is not possible to tell whether the observed effect was due to selective adaptation of /b/ (lower /b/ variance), or recalibration of /v/ (shift in /v/ mean), since both would produce a shift in the category boundary towards $/ \mathrm{b} /$. Unless they completely ignored the audio component, subjects in this experiment likely perceived the audio-visual adaptor not as a perfect /va/, but rather

\footnotetext{
${ }^{4}$ An earlier study (Roberts \& Summerfield, 1981) used an audio-/ba/, visual-/ga/ adaptor. This was intended to produce a percept of /da/ (as in McGurk \& J. MacDonald, 1976), but only half of their subjects perceived an alveolar sound at all, with the others reporting $/ \mathrm{kl} /, / \mathrm{m} /$, or /fl/ (Saldaña \& Rosenblum, 1994). This makes it difficult to interpret their results and so we do not discuss them further here.
} 
a somewhat /ba/-like /va/. Kleinschmidt and Jaeger (2011) presented evidence that phonetic distributional learning operates on this kind of combined audiovisual percept (also cf. Bejjanki, Clayards, et al., 2011; Ernst \& Bülthoff, 2004). If this is in fact the case, then listeners in Saldaña and Rosenblum's experiment should update their beliefs about the / $/$ / cue distribution, shifting it to be more /b/ like. The result of this recalibration of / $/$ would be more $/ \mathrm{v} /$ and hence fewer /b/ responses, which is the after-effect that Saldaña and Rosenblum (1994) observed. Thus, the results of Saldaña and Rosenblum (1994) are not necessarily incompatible with distributional learning at the phonetic category level.

We believe that future work addressing this question will prove particularly valuable in evaluating the feasibility of an explanation of selective adaptation purely in terms of distributional learning. For instance, we have suggested that the after-effects observed by Saldaña and Rosenblum (1994) are due to recalibration to an integrated percept that combines audio and visual cues. This account predicts that changing the reliability of the visual cue should change the integrated percept (as observed by Bejjanki, Clayards, et al., 2011; Ernst \& Bülthoff, 2004), and thus the resulting after-effect, while the audio-only selective adaptation account offered by Saldaña and Rosenblum (1994) predicts that changing the visual cue should have no effect at all.

\subsection{Discussion}

Since selective adaptation for speech was first discovered, our understanding of both adaptation in sensory systems in general, and flexibility in the language system in particular has come a long way. First, work on non-linguistic sensory adaptation has established that adaptation goes far beyond fatigue of feedforward feature detectors (Brenner et al., 2000; Dragoi et al., 2000; Fairhall et al., 
2001; Gutfreund, 2012; Kohn \& Movshon, 2004; Kohn, 2007; Sharpee et al., 2006; Webster et al., 2005). In particular, there is tentative evidence that sensory adaptation serves to increase the efficiency of the representation of sensory information, based on the statistical or distributional properties of recent sensory input. Second, the speech perception system is now understood to be flexible in smart ways, for example, by recalibrating phonetic categories based on unusual pronunciations (Bertelson et al., 2003; Kraljic \& Samuel, 2005; Norris et al., 2003).

Both of these developments are rooted in a growing awareness of the importance of distributional or statistical properties of the sensory world for understanding sensory systems. While these two developments may appear rather dissimilar-one concerned with the allocation of scarce neural resources, and the other with robust language comprehension in the presence of variability-they come together in the phenomenon of phonetic selective adaptation. Although for different reasons than originally anticipated, selective adaptation thus rightly deserves the spotlight it enjoyed in the early era of cognitive research on speech perception. Moreover, we have tentatively proposed that selective adaptation can be understood as one consequence of the more general process of distributional learning, along with phonetic recalibration and other forms of smart adaptation in language processing (Kleinschmidt \& Jaeger, 2015). This proposal provides good coverage of the data that exists on how selective adaptation changes listeners' classification of speech sounds.

More importantly, however, we hope that this proposal will provide a road map for how specifically to go about re-evaluating selective adaptation. There is a great deal of work that is required to flesh out and critically evaluate such a distributional-learning theory. In closing, we discuss four important directions for future research. 


\subsubsection{Future directions}

First, distributional learning is a computational-level account, in the sense of Marr (1982). Such accounts focus on the in-principle constraints on a cognitive system that come from the information that is available and the task the system is carrying out. While these considerations guide and constrain cognitive models, they are not cognitive models per se. There are many different possible algorithms, processes, or representations that can carry out the computation of distributional learning, and many possible neural implementations for each of those. Fleshing out the relationships between these is an important next step in formulating and evaluating a complete distributional learning theory of selective adaptation. Critically, mechanistic- and computational-level explanations are not necessarily in opposition. It may well be the case, for instance, that a population of fatiguing feature detectors can approximate distributional learning in some circumstances. These links are, however, largely un-explored, and call for future work.

Second, and relatedly, we have focused on the effects of selective adaptation on listeners' classification decisions, and have not discussed reaction time effects of selective adaptation. Such effects are well-documented (Samuel, 1986; Samuel \& Kat, 1996) and historically one of the most important sources of insight into selective adaptation. Across the cognitive sciences, the outcome of a decision can often be dissociated from the time course of that decision (see, e.g., the related discussion for lexical selection in speech production; Mahon, Costa, Peterson, Vargas, \& Caramazza, 2007; Oppenheim, Dell, \& Schwartz, 2010). The same is true in phonetic selective adaptation: two adaptors can produce the same boundary shifts, but different changes in reaction time (Samuel \& Kat, 1996). A computational-level account like distributional learning naturally captures the decisions that listeners make, but the time course falls more under the purview of process- or implementation-level accounts. Thus, a crit- 
ical next step in evaluating distributional learning as a theory of adaptation in speech perception (and perception more broadly) is to develop process- and implementation-level models that carry out the computations of distributional learning. There is some promising if preliminary work that links increased probability of a stimulus to an increase in signal-to-noise ratio in sensory representations (Stocker \& Simoncelli, 2006; Wei \& Stocker, 2012). Increased reaction times might be a consequence of achieving such increased signal-to-noise ratio through, for instance, stronger lateral inhibition leading to sparser responses by effectively narrowing receptive fields (Gardner et al., 2004). We emphasize that this is only one possibility, and that in general linking distributional learning to reaction time data at all is a critical direction for future work.

Third, as we have outlined it here, distributional learning accounts for selective adaptation through changes in listeners' beliefs about the variance of the adapted phonetic category. There is some evidence that listeners are, in fact sensitive to category variance (Clayards et al., 2008; Cole \& Cooper, 1977; Newman et al., 2001; Schreiber, Onishi, \& Clayards, 2013), but only a few studies have explored this systematically. Only one study that we know of has specifically investigated the link between category variance and selective adaptation (Cole \& Cooper, 1977), and while, as we have argued, their results are consistent with a distributional learning theory, their design confounds category variance and category mean. More work is required, first to determine what distributional learning predicts the effect of different degrees of category variance is, and second to see whether listeners actually behave in the predicted ways.

Fourth, distributional learning provides a bridge between selective adaptation-typically thought of as a low-level process-and higher-level processes like talker-specific recalibration and accent adaptation. A hallmark of these processes is that listeners often apply what they have previously learned to 
future situations in smart ways. This can mean recognizing a previously encountered talker, as demonstrated by recalibration that persists even after 12 hours outside the lab (Eisner \& McQueen, 2006). It can also mean generalizing learning to an unfamiliar talker that is similar to previously-encountered talkers, as demonstrated by talker-independent accent adaptation (Bradlow \& Bent, 2008). Elsewhere (Kleinschmidt \& Jaeger, 2015), we have proposed that such cross-situational learning effects can be modeled as distributional learning that is indexed to particular indexical variables like a talker, groups of talkers, or environment. Such hierarchical distributional learning enables previously learned cue distributions to be re-learned very quickly when the associated indexical variable is encountered again (for similar arguments applied to domain-general sensory / motor learning, see Qian et al., 2012, 2015). If selective adaptation is due to the same sort of distributional learning, then it follows that listeners should re-adapt to previously encountered distributions more quickly than the initial adaptation. To our knowledge, this prediction has not been addressed in the literature on phonetic selective adaptation.

\subsubsection{Whither feature detectors?}

We have focused on-and argued against— the assumption that phonetic selective adaptation is the fatigue of auditory-phonetic feature detectors, without discussing the nature or existence of these feature detectors themselves. The early work on selective adaptation was rightly criticized for relying on problematic assumptions about these feature detectors (Remez, 1987): that there were a small number of them, specified largely a priori, which respond largely independently of context, and are organized into clearly delineated layers (simple acoustic, complex acoustic, phonetic, etc.). Our proposal that selective adaptation be seen as a form of distributional learning does not rely on these same assumptions. Nevertheless, a fully fleshed out theory of adaptation as 
distributional learning has to say something about what those distributions are of. The things that these distributions cover may be seen as a sort of features, and expressing those distributions in terms of some relatively low-dimensional compression of the full acoustic input information makes sense for the same reasons that distributional learning makes sense: sensory systems have limited representational resources, and care about some aspects of the sensory world more than others. But there is no guarantee that these "features" will line up with our intuitions about what the relevant features are, or that they will be organized in clearly delineated levels.

There is also no guarantee that adaptation itself provides a straightforward means of probing these features. In fact, the work on visual adaptation we reviewed here suggests that adaptation has to be considered in terms of distributions across multiple levels simultaneously. We take these levels to refer not only to part-whole feature relationships (He et al., 2012), but also to the statistical sense of distributions over the same features but at different time scales (seconds vs. minutes) (Chopin \& Mamassian, 2012) or groups of situations (talker, accent, language, etc.). There is analogous work in speech perception, including selective adaptation, that makes the same point (e.g., adaptation of single segments depends on the syllabic context Bryant, 1978; and others summarized in Remez, 1987), but the consequences of adaptation across multiple levels have not been fully explored, either in vision or speech perception.

\subsubsection{Speech as a model organism for perception}

Finally, we close by arguing that selective adaptation provides a good bridge between speech perception and the study of perception more generally. As we have discussed, recent work on general sensory adaptation has revealed that adaptation has a variety of functional properties that make it interesting as an 
object of study in its own right, rather than just a methodological tool. The emerging computational understanding of adaptation puts it squarely at the intersection of many fundamental and interesting questions in the study of perception, such as the interaction between bottom-up and top-down information, the ways that innate constraints and information from the world shape perceptual systems, the role that probabilistic predictions play in perception (e.g., He et al., 2012), and how the brain manages to process sensory information efficiently in a variable world with a limited stock of representational resources, among others.

Speech perception exemplifies many of these issues as well as, or better than, other perceptual processes. As a sensory signal, speech is structured in multiple, intersecting ways. On the one hand, there is the linguistic structure of speech: individual sounds combine into phonetic categories, categories into words, words into phrases, phrases into sentences, etc. On the other hand, there is the indexical structure of speech: every talker maps phonetic categories to sounds in a different way, often dramatically so, and talkers themselves can be clustered based on dialect, native language background, etc. While neither sort of structure is exhaustively understood, they are understood well enough to manipulate them experimentally in ways that carefully control their statistical properties but are reasonably ecologically valid (Allen \& J. L. Miller, 2004; Clayards et al., 2008; Newman et al., 2001). This stands in contrast to visual psychophysics, which in large part relies on simple, artificial stimuli in order to achieve careful control. Obviously, much has been learned from these techniques. But for investigating questions of how sensory systems deal with changes in the statistics of the sensory world, more leverage might be provided by studying sensory domains where such deviations occur frequently and are typically managed successfully by human observers. Speech perception is just such a domain: differences between talkers are well-studied, often to the extent 
of quantifying the statistical deviations from one talker to another. Likewise, the last decade has seen an explosion in research showing listeners can rapidly adapt to different talkers.

What role does phonetic selective adaptation play in all of this? It sits at the intersection of the study of low-level adaptation in vision and more recent work on smart plasticity in speech perception and language comprehension. It is our hope that, by bringing new attention to the long literature on phonetic selective adaptation, researchers studying speech perception will be more aware of developments in how sensory adaptation more broadly is understood, and that researchers who study perception in general will consider both the potential offered and the challenges posed by speech perception as a perceptual process.

\subsubsection{Conclusion}

The literature on phonetic selective adaptation is long, complex, and often apparently contradictory (as summarized by Samuel, 1986; and Remez, 1987). Over time, interest in the theoretical basis for this fundamental phenomenon has dwindled. We believe, though, that adaptation is an important property of perception in general, and of speech perception in particular, and that this literature deserves more attention. Recent developments in the understanding of sensory adaptation more broadly and flexibility in speech perception in particular challenge some of the basic assumptions of early work on phonetic selective adaptation. However, as we have argued, they also provide a set of conceptual tools for understanding phonetic selective adaptation in the broader context of language comprehension and sensory adaptation. We have proposed, to start, that selective adaptation can be seen as a form of distributional learning. Distributional learning provides a coherent perspective on the existing literature on phonetic selective adaptation, highlights parallels with 
non-speech adaptation, and suggests a unifying perspective on flexibility in adult language comprehension. It also, most importantly, raises a number of interesting questions for future work. 


\section{What do you expect from an unfamiliar talker? Inferring listeners' priors beliefs ${ }^{1}$}

A longstanding problem in speech perception is how listeners manage to cope with substantial differences in how individual talkers produce speech. Recent evidence suggests that one strategy listeners employ is to rapidly adapt to unfamiliar talkers (Bertelson et al., 2003; Clarke-Davidson \& Garrett, 2004; Kraljic \& Samuel, 2007, among others). Such adaptation can be understood as a form of statistical inference. This insight is captured by a recent proposal, the ideal adapter framework (Kleinschmidt \& Jaeger, 2015). Each talker's particular accent (way of talking) can be formalized as the distribution of acoustic cues that they produce for each phonetic category (or other underlying linguistic unit). Listeners are taken to adapt to an unfamiliar talker via distributional learning, inferring the underlying talker-specific cue distributions from the talker's productions.

Critically, this statistical inference process draws on implicit beliefs about

\footnotetext{
${ }^{1}$ This work was previously published as Kleinschmidt, D. F. \& Jaeger, T. F. (2016b). What do you expect from an unfamiliar talker? In J. Trueswell, A. Papafragou, D. Grodner, \& D. Mirman (Eds.), Proceedings of the 38th annual meeting of the cognitive science society. Austin, TX: Cognitive Science Society
} 
how talkers tend to differ from each other. As a consequence, in this view adaptation to an unfamiliar talker depends on a listener's prior experience with other talkers, rather than only on the speech produced by the unfamiliar talker themselves. Specifically, a listener's experience with other talkers provides the starting point for the distributional learning required for adaptation, or, in Bayesian terms, a prior belief about the probability of different possible accents (cue distributions). More informative prior beliefs can substantially reduce the amount of direct evidence needed to converge on accurate beliefs about the current talker's cue distributions.

The goals of the present work are two-fold. First, we test a critical prediction of the ideal adapter framework. To the extent that a listener's prior beliefs are informative, they must take some probability away from unlikely accents. Confronted by a talker whose accent falls well outside the range of what they expect based on their previous experience, the ideal adapter framework predicts that a listener will require more evidence to adapt, leading to slowed or incomplete adaptation.

There is already some evidence for this prediction. For instance, Idemaru and Holt (2011) tested how well listeners adapt to distributions of two cues that distinguish voicing (e.g., /b/ vs. / p/), voice onset time (VOT, the primary cue to voicing) and the pitch of the following vowel (f0, a secondary cue). These two cues are typically positively correlated in English, with /p/ corresponding to high VOT and high f0, and /b/ to low values of both cues (Kohler, 1982). In one condition, listeners were exposed to a talker who produced a positively correlated distribution of these cues. During a post-test, these listeners used f0 to categorize stops with ambiguous VOTs. In another condition, listeners heard a talker who produced an uncorrelated distribution, where $\mathrm{f} 0$ is uninformative. In contrast to the listeners in the first condition, during the post test these listeners ignored f0 even for ambiguous VOTs. This effect is consistent 
with the idea that listeners are rationally integrating multiple cues to voicing, weighing them based on how informative they are (Ernst \& Bülthoff, 2004; Clayards et al., 2008; Bejjanki, Clayards, et al., 2011). However, listeners in a third condition who were exposed to a talker who produced an anticorrelated did not follow the predictions of rational cue integration. Despite the fact that f0 was just as informative for this accent as for the positively correlated accent, listeners ignored $\mathrm{f} 0$ as a cue to voicing. This suggests that these listeners have ruled out the possibility of a reversed mapping between $\mathrm{f} 0$ and voicing (/b/ vs. /p/), possibly perhaps American English talkers typically do not typically produce it (e.g., House, 1953). Likewise, Sumner (2011) found that listeners had trouble adapting to a talker who produced VOT distributions for $/ \mathrm{b} /$ and $/ \mathrm{p} /$ that had substantially lower means (approximately -60ms and 0ms, respectively) than a typical talker (approximately $0-10 \mathrm{~ms}$ and $60 \mathrm{~ms}$ VOT; Lisker \& Abramson, 1964).

These results are consistent with the ideal adapter's prediction that listeners use gradient, structured knowledge about how talkers tend to vary to guide their adaptation to an unfamiliar talker. However, it is also possible that these results are simply due to the extremely unnatural input that listeners received. Both studies involved gross, categorical mismatches between typical and experimental cue distributions: a reversal of the f0-to-voicing mapping in Idemaru and Holt (2011), and a remapping of /b/-like VOTs to /p/ in Sumner (2011).

Our goal first goal in this paper is thus a stronger test of the ideal adapter hypothesis. In two experiments, we expose listeners to a range of different accents, which differ (only) in the distributions of voice onset time (VOT). By parametrically manipulating these distributions, we create a range of accents that are more or less similar to what a typical talker of English produces. We then assess the degree to which listeners adapt their beliefs about the novel 
talker's cue distributions, depending on the a priori typicality of these distributions.

To anticipate the results, we find that typicality of the novel talker's cue distribution predicts the degree to which listeners adapt to the talker. This suggests that listeners not only have beliefs about the cue distributions for a particular single talker (as suggested by previous work, Clayards et al., 2008; Feldman, Griffiths, \& Morgan, 2009; Kleinschmidt \& Jaeger, 2015; Kronrod et al., 2012), but also have implicit beliefs about the ways in which talkers tend to differ from each other, and hence what to expect from an unfamiliar talker.

This leads to the second question we address here: what is the content of listeners' prior beliefs about inter-talker variability? To this end, we present an inverted belief-updating model, which allows us to work backwards from listeners' adaptation behavior across talkers in order to infer listeners' shared prior beliefs. This approach has a number of advantages. First, it provides a more direct assessment of listeners' subjective prior beliefs, which are, according to the ideal adapter, what matters for understanding adaptation. Second, it allows us to compare these subjective beliefs about talker variability to data on the objective level and type of cross-talker variability that is present in the world. Third, by assessing listeners prior beliefs directly from their behavior, this method avoids the need for production data, which is difficult to collect and often requires time-consuming expert annotation.

If this model is successful, it provides a missing methodological tool for understanding listeners' prior expectations. These expectations are, according to the ideal adapter, one of (if not the) most important factor in listeners' remarkable ability to efficiently understand speech from many different talkers. In particular, to fully understand listeners' prior beliefs we need to understand how they are learned from experience with actual, objective cross-talker variability, how they are structured across different cues/categories, and how they relate 
to socio-indexical grouping variables like gender, dialect, etc. This modeling work thus serves as a proof-of-concept for future work on these questions.

We first present the design and results of Experiment 1. Then we use a belief updating model to infer listeners' prior beliefs based on the results of that experiment. Finally, we present the results of Experiment 2 which uses a wider range of distributions, and assess how well the inferred prior beliefs predict listeners' adaptation behavior in Experiment 2.

\subsection{Experiment 1}

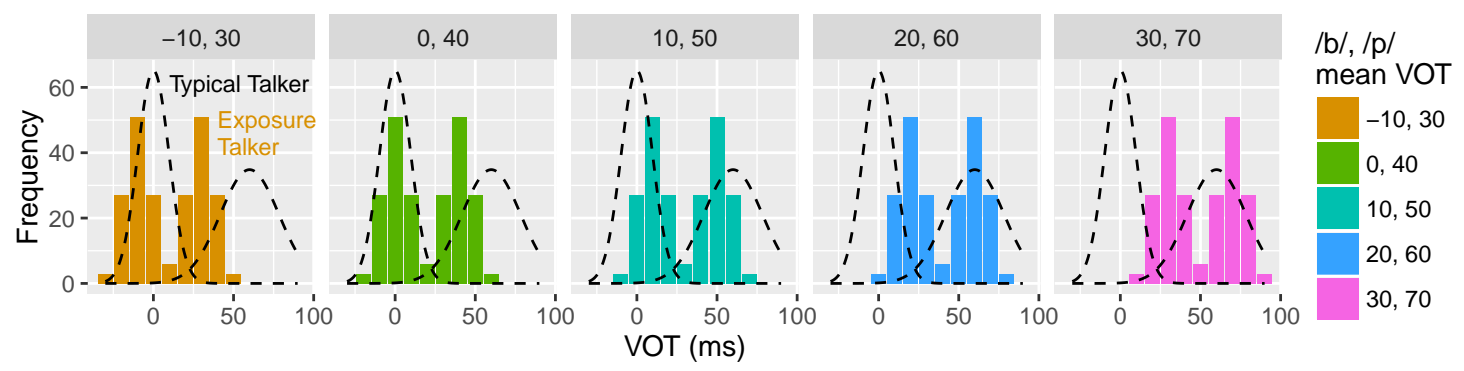

Figure 4.1: Each subject heard one of these five synthetic accents, which differ only in the distribution of VOTs of the word-initial stops. Black dashed lines show VOT distributions from a hypothetical typical talker (as estimated by Kronrod et al., 2012). Note that the 0 and $10 \mathrm{~ms}$ shifted accents are reasonably close to this typical talker, while the $-10,20$, and $30 \mathrm{~ms}$ shifted accents deviate substantially.

We tested the role that listeners prior expectations play in adapting to an unfamiliar talker by exposing them (between subjects) to one of five synthetic "accents" (Figure 4.1). These accents differed only in the distribution of voice onset time (VOT), the primary cue to word-initial stop consonant voicing in English (e.g., "beach" vs. "peach"). Adaptation was assessed based on listeners' classification function, or how they labeled each VOT as $/ \mathrm{b} /$ or $/ \mathrm{p} /$, which was continuously assessed throughout the experiment. 
These conditions vary in how similar they are to a typical talker's VOT distributions. The $0 \mathrm{~ms}$ and $10 \mathrm{~ms}$ VOT $/ \mathrm{b} /$ mean distributions are particularly similar to the typical talker's cue distribution (Figure 4.1, black dashed lines), while the other conditions diverge substantially.

For a typical talker, we use the distributions reported by Kronrod et al. (2012), who determined the underlying means and variances that best explained a combination of listeners' VOT classification and discrimination behavior. These distributions are qualitatively very similar to those reported by Lisker and Abramson (1964), based on productions from four talkers. The reason we use the estimates from Kronrod et al. (2012) is because Lisker and Abramson (1964) only report the mean VOT for each category, and not the variance, which is necessary to predict the classification function.

\subsubsection{Methods}

\section{Subjects}

We recruited 169 subjects via Amazon's Mechanical Turk. Subjects were paid $\$ 2.00$ for participation, which took about 20 minutes. We excluded subjects who participated more than once $(n=4)$ or who failed to classify VOTs reliably ( $n=28 ; n=1$ for both reasons). We defined reliable classification as accuracy of at least $80 \%$ at 0 and $70 \mathrm{~ms}$ VOT. Because some conditions had few stimuli with these VOTs, we extrapolated subjects' responses using a logistic generalize linear model (GLM). Excluded subjects were roughly equally distributed across conditions (maximum of 5 in $0 \mathrm{~ms} / \mathrm{b} /$ VOT condition, and minimum of 1 in $20 \mathrm{~ms} / \mathrm{b} / \mathrm{VOT}$ condition). After these exclusions, data from 138 subjects remained for analysis. 

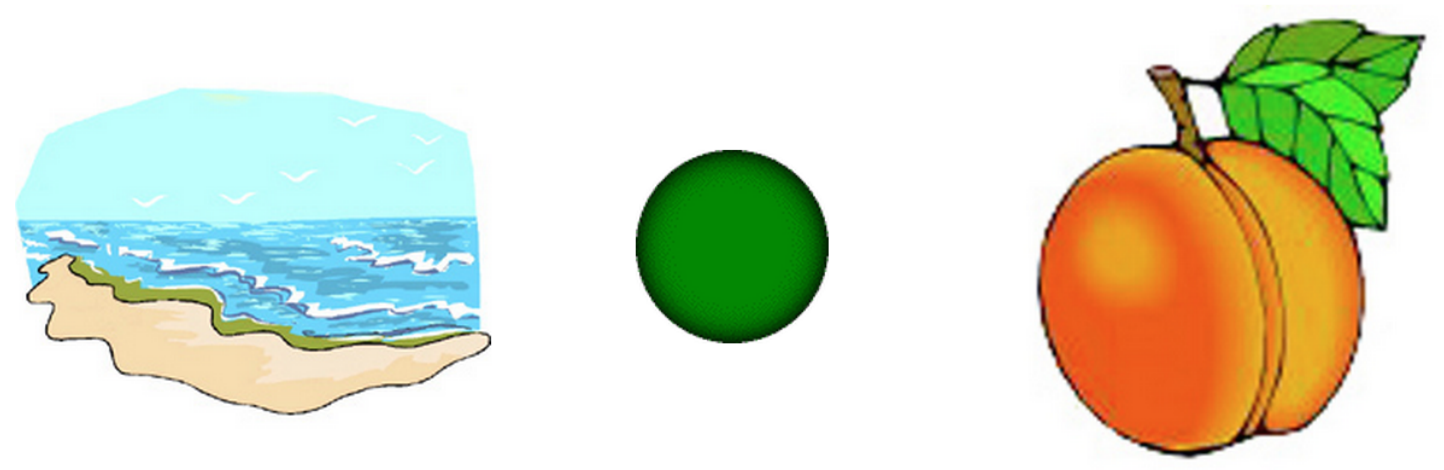

Figure 4.2: Example trial display (beach/peach). Listeners first click on the green button to play the word, then click on one picture to indicate what they heard.

\section{Procedure}

Our procedure is based on Clayards et al. (2008). Figure 4.2 shows an example trial display. On each trial, two response option images appeared, which corresponded to one of three /b/-/p/ minimal pairs (beach/peach, bees/peas, or beak/peak). Subjects started each trial by clicking on a button between the two pictures, which played the corresponding minimal pair word audio stimulus. Subjects then clicked on the picture to indicate whether they heard the /b/ or /p/ member of the minimal pair. Subjects performed 222 of these trials, evenly divided between the three minimal pairs, in random order.

Each trial's word was synthesized with a voice onset time (VOT) that was randomly drawn from a bimodal distribution, with low and high VOT clusters implicitly corresponding to /b/ and / $\mathrm{p} /$, respectively. This distribution defined the accent that the subject heard, and each subject was pseudorandomly assigned to one of five accent conditions (Figure 4.1). 


\section{Materials}

The audio and visual stimuli we used were identical to those in Clayards et al. (2008). Three /b/-/p/ minimal pair audio continua were synthesized using the 1988 Klatt synthesizer (Klatt, 1980), by manipulating VOT in 10ms increments (either adding voicing before the stop burst to create negative VOTs, or aspiration after for positive VOTs). Within a $/ \mathrm{b} /-/ \mathrm{p} /$ continuum, the other parameters were held constant, and modeled on natural tokens of the endpoints (beach/peach, bees/peas, and beak/peak).

\subsubsection{Results and Discussion}

To assess how well listeners adapted to the exposure talker's cue distributions, we estimated listeners' classification functions, and compared those to the typical and exposure talker's classification functions. Each listener's classification function was estimated by fitting a logistic GLM to the proportion of $/ \mathrm{p} /$ responses given VOT. The classification functions for the typical and exposure talkers' distributions were determined using an ideal listener model (Clayards et al., 2008; Kleinschmidt \& Jaeger, 2015). Under such a model, the predicted probability of a / $\mathrm{p} /$ response for a VOT of $x$, denoted $p(c=\mathrm{p} \mid x)$ is the likelihood of $x$ under the / $\mathrm{p} /$ distribution, $p(x \mid c=\mathrm{p})$, divided by the total likelihood under both $/ \mathrm{b} /$ and $/ \mathrm{p} /, p(x \mid c=\mathrm{b})+p(x \mid c=\mathrm{p})$.

Using the cue distributions for the typical talker, this provides an estimate of how listeners would classify VOTs if they were completely insensitive to the exposure talker. Using, on the other hand, the distributions produced by the exposure talker provides an estimate of how listeners would classify VOTs if they completely adapted to the exposure talker, ignoring any prior beliefs they may have. Such complete adaptation is not necessarily optimal, especially given limited input data. The ideal adapter predicts that listeners' classification func- 
tions will be intermediate between these two extremes, reflecting a compromise between prior beliefs and current exposure.
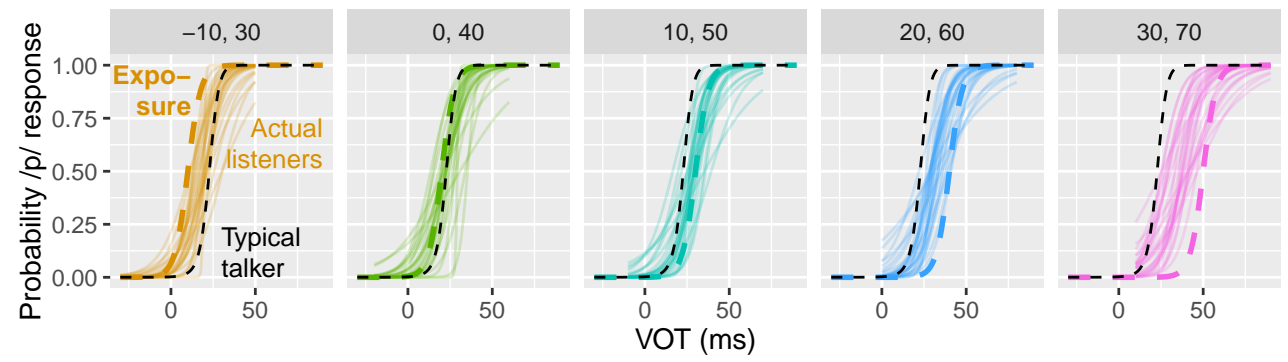

$$
\begin{aligned}
& \text { /b/, /p/ } \\
& \text { mean VOT } \\
& -\quad-10,30 \\
& -\quad 0,40 \\
& -\quad 10,50 \\
& -\quad 20,60 \\
& -\quad 30,70
\end{aligned}
$$

Figure 4.3: Listeners' responses, smoothed with logistic functions (thin lines), compared with the classification functions expected based on a typical talker (no learning; dashed black lines) and complete (but not necessarily optimal) adaptation to the exposure distributions (thick dashed colored lines). Listeners' actual category boundaries lie between the typical talker and exposure talker boundaries (see Table 4.1).

Figure 4.3 shows the classification functions for each individual listener. In each accent, these classification functions tend to fall in between the boundaries predicted by the typical talker distributions and the boundaries implied by the exposure distributions. Figure 4.4 shows this directly, by plotting the distribution of individual listeners' $/ \mathrm{b} /-/ \mathrm{p} /$ boundaries, relative to the exposure and typical talker's boundaries.

We can quantify how much listeners shifted their category boundaries by the percentage of the predicted shift in category boundary from the classification function for the typical talker to the boundary implied by the input distribution (Table 4.1). A $0 \%$ shift corresponds to no adaptation at all, while a shift of 100\% corresponds to complete adaptation to the exposure distributions, with no (remaining) influence of any prior beliefs.

In all conditions, the average shift percentage was between $0 \%$ and $100 \%$ (except the 0ms shift condition, which is so close to the typical talker that estimating the percentage is numerically unstable). More interestingly, the more extreme conditions show less complete adaptation than the less extreme condi- 


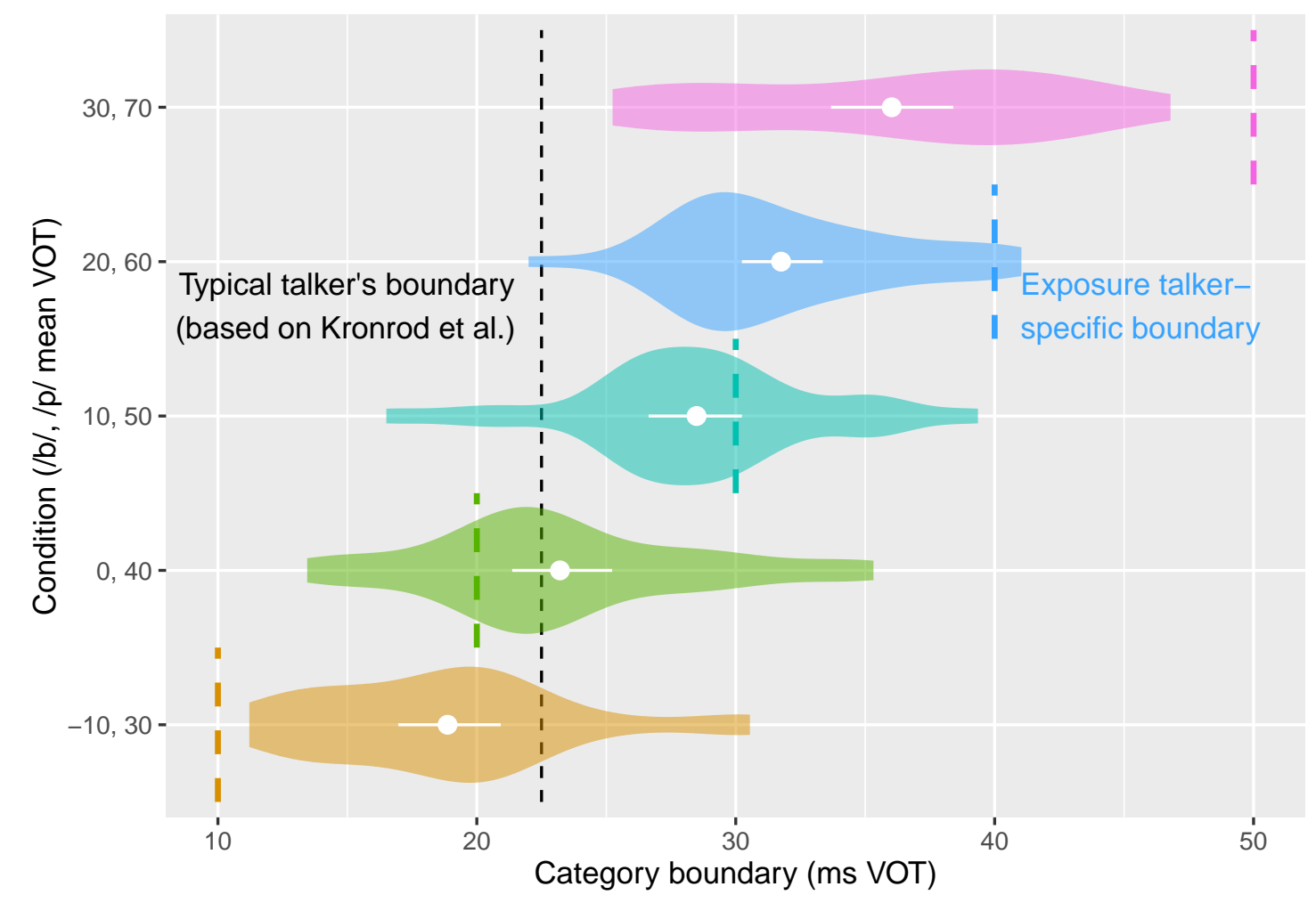

Figure 4.4: Most listeners' individual boundaries fall between the boundaries implied by cue distributions from a typical talker and exposure talker. Violin plots (shaded regions) show the density of individual subjects' category boundaries. White points and CIs show mean and bootstrapped 95\% CIs for mean boundary in each condition. 
tions. Together, these results suggest that listeners' adaptation was constrained by their prior expectations (given the finite amount of evidence they received about the unfamiliar talker). This provides qualitative evidence that listeners combine their prior expectations with observed cue distributions in order to rapidly adapt to unfamiliar talkers, as predicted by the ideal adapter framework (Kleinschmidt \& Jaeger, 2015). ${ }^{2}$ More generally, unlike previous work showing constraints on distributional learning (Idemaru \& Holt, 2011; Sumner, 2011), these results cannot be explained by a model where the only constraints on listeners' distributional learning is a binary distinction between natural and unnatural cue-category mappings.

Table 4.1: Percentage of boundary shift from typical talker to each exposure talker (see Figure 4.3), averaged over subjects with $95 \%$ bootstrapped confidence intervals. $0 \%$ shift corresponds to no adaptation at all, while $100 \%$ corresponds to perfect adaptation, ignoring any prior beliefs. Typical and exposure talker boundaries were too close together to reliably determine boundary shift percentage in the $0 \mathrm{~ms}$ condition.

\begin{tabular}{lll}
\hline$/ \mathrm{b} /, / \mathrm{p} /$ mean VOT & Mean shift & $95 \% \mathrm{CI}$ \\
\hline$-10,30$ & $29 \%$ & $14-43 \%$ \\
0,40 & - & - \\
10,50 & $80 \%$ & $57-104 \%$
\end{tabular}

\footnotetext{
${ }^{2}$ It is not immediately clear whether these results are compatible with exemplar accounts of speech perception (Pierrehumbert, 2001; Johnson, 1997a; Goldinger, 1998). On the one hand, these models broadly predict that listeners will adapt their categorization based on experience, and that this is guided by previous experience because previously stored exemplars are used to categorize each new exemplar. On the other hand, to our knowledge there are no existing studies that model unsupervised distributional learning with exemplar models. This is thus a question for future work based on an implemented exemplar model.
} 


\begin{tabular}{lll}
\hline$/ \mathrm{b} /, / \mathrm{p} /$ mean VOT & Mean shift & $95 \% \mathrm{CI}$ \\
\hline 20,60 & $53 \%$ & $44-62 \%$ \\
30,70 & $49 \%$ & $40-58 \%$ \\
\hline
\end{tabular}

\subsection{Inferring prior beliefs about talker variability}

Our second goal in this paper is to test whether it is possible to infer listeners' prior beliefs about talker variability, based on their patterns of adaptation to different accents. To that end, we use a variant of a Bayesian belief-updating model that has previously provided a good account of how listeners incrementally update their beliefs in order to rapidly adapt to an unfamiliar talker (Kleinschmidt \& Jaeger, 2015). This previous modeling work has treated the content of listeners prior beliefs-the category means and variances they think are most likely - as known and fixed, setting them based on pre-adaptation classification data, and then fitting the confidence in those prior beliefs as a free parameter. The goal of this earlier modeling work was to test how well belief updating itself could explain the rapid build up of adaptation, and fixing the prior expected category means and variances restricted the

Here, we wish to fit both the content of (prior expected mean and variance of each category) and the confidence in prior beliefs, based on adaptation data presented above.

Kronrod et al. (2012) also use a Bayesian model to estimate the underlying distributions of VOT for $/ \mathrm{b} /$ and $/ \mathrm{p} /$ based on comprehension behavior. Specifically, they fit the mean and variance of each category directly to listeners' classification function for a VOT continuum. ${ }^{3}$ However, despite this superficial

\footnotetext{
${ }^{3}$ They also estimated a sensory uncertainty, or noise variance, parameter, based on discrimination of pairs of VOTs at different points on the VOT continuum. The primary goal of their
} 
similarity, their approach is very different from ours. Their estimates are based on listeners' perception of speech from a single talker, who moreover produced a highly unnatural flat distribution over a wide range of VOTs. Thus, the resulting estimates of the underlying distributions reflect, at most, listeners' beliefs about this single, highly unusual talker's production of VOT, and don't support any conclusions about cross-talker patterns at the population level. While this might also seem to invalidate our use of their estimates as a "typical" talker, the distributions they inferred bear a close resemblance to those from Lisker and Abramson (1964). This suggests that listeners in their experiment may have discounted the unusual, flat VOT distribution they experienced as too unusual to be representative of the talker's actual distributions.

\subsubsection{Methods}

We model each category $(/ \mathrm{b} /$ and $/ \mathrm{p} /$ ) as a single normal distribution. Listeners' uncertain beliefs about the underlying cue distributions can thus be modeled as a probability distribution over the parameters of the category distributions, which we denote

$$
\theta=\left\{\mu_{\mathrm{b}}, \sigma_{\mathrm{b}}^{2}, \mu_{\mathrm{p}}, \sigma_{\mathrm{p}}^{2}\right\}
$$

where $\mu_{c}$ is the mean VOT of category $c$ and $\sigma_{c}^{2}$ is its variance. We do not include the mixing weight (category prior probability) as a parameter, assuming that each category is equally likely. This is true in the context of our experiment, and moreover the only effect of unequal weights is a constant shift in the category boundary, regardless of the distributions (Feldman, Griffiths, \& Morgan, 2009). As in Kleinschmidt and Jaeger (2015), we use an independent, conjugate Normal- $\chi^{-2}$ prior for each category, with parameters $\phi$ (Gelman, Carlin, et al.,

study was to investigate the relative contribution of intrinsic category variability and sensory uncertainty in listeners' perception. We lump both of these factors together in this study, because their influence cannot be disentangled based on classification alone. 
2003)

$$
\begin{aligned}
\phi & =\left\{\mu_{0, \mathrm{~b}}, \sigma_{\mathrm{b}}^{2}, \mu_{0, \mathrm{p}}, \sigma_{\mathrm{p}}^{2}, \kappa_{0}, \nu_{0}\right\} \\
\theta \mid \phi & \sim \prod_{c \in\{\mathrm{b}, \mathrm{p}\}} \text { Normal }\left(\mu_{c} \mid \mu_{0, c}, \frac{\sigma_{c}^{2}}{\kappa_{0}}\right) \chi^{-2}\left(\sigma_{c}^{2} \mid \sigma_{0, c}^{2}, v_{0}\right)
\end{aligned}
$$

where $\mu_{0, c}$ and $\sigma_{0, c}^{2}$ are the prior expected mean VOT and variance of category $c$, respectively, and $\kappa_{0}$ and $v_{0}$ are the listener's confidence in these prior expectations. In this parametrization, the prior confidence parameters $\kappa_{0}$ and $v_{0}$ can be interpreted as pseudo-counts, or the number of hypothetical observations that listeners' prior beliefs about the category means and variances are based on. Note that, as in our previous modeling work, these prior confidence parameters are shared between the two categories. That is, we assume that listeners have equal confidence in their prior beliefs about the mean of $/ \mathrm{b} /$ and of $/ \mathrm{p} /$ (and likewise for the variance of $/ \mathrm{b} /$ and $/ \mathrm{p} /)^{4}$

To estimate the listeners' prior beliefs, we infer values for these parameters given the observed adaptation behavior (category responses $y$ and input VOTs $x$ ) using Bayesian inference, marginalizing over the actual category means and variances $\theta$ :

$$
\begin{aligned}
p(\phi \mid x, y) & \propto p(y \mid \phi, x) p(\phi) \\
& \propto \int \mathrm{d} \theta p(y \mid \theta, x) p(\theta \mid x, \phi) p(\phi)
\end{aligned}
$$

We make two simplifying assumptions. First, we assume that the order of the trials does not matter. ${ }^{5}$ Second, we assume that listeners pick up on the

\footnotetext{
${ }^{4}$ This is not an in-principle limitation of this framework, but rather a limitation of the data. Preliminary simulations showed that it wasn't possible to uniquely identify the model using separate prior confidence parameters for the two categories, with the posterior distributions of the two category's confidence parameters given the data highly correlated.

${ }^{5}$ The order of trials does matter, in general (Vroomen, van Linden, de Gelder, \& Bertelson, 2007; Kleinschmidt \& Jaeger, 2015; Kraljic, Samuel, \& Brennan, 2008). In particular, our previous modeling work models such trial effects. However, the paradigm that this previous work
} 
cluster structure of the input they receive, updating their beliefs about each category based on the true mean and variance of the corresponding cluster, and classify each trial's VOT based on these updated beliefs. These two assumptions together mean that, given the prior belief parameters, we can express the predicted classification function (that is, probability of $/ \mathrm{p} /$ for each VOT) in closed form, which makes model fitting substantially more tractable.

At first blush, our second assumption seems to leave a lot of information in our dataset on the table, since we don't consider listeners' own judgments about the category of each observation when modeling how beliefs are updated. It is possible to back off on this assumption, but in exploring this we found that the question of how to incorporate listeners' judgments into the belief updating model to be surprisingly subtle. For instance, one seemingly obvious fix that we investigated is to treat listeners' responses as the category labels to be used for belief updating (rather than the experimenter-defined categories). However, this technique effectively fits the exposure distributions to listeners' classification functions, and in doing so biases the model to completely ignore the prior.

Moreover, this approach has conceptual problems, because by treating listeners' responses to earlier trials as fixed it eliminates the model's ability to go back and re-evaluate previous judgments. This is not necessarily an unreasonable assumption, and similar ideas have been proposed as resource-limited approximate inference strategies (Sanborn et al., 2010). But such approaches would complicate the interpretation of this initial study of inferring listeners' prior beliefs, because they conflate the basic assumptions of the inverted belief-

models is very different, with a small number of critical trials that are maximally ambiguous. Such recalibration paradigms produce very rapid adaptation. The build-up time course of the distributional learning paradigm we use here has not been studied in detail, but what evidence exists suggests that the effects are much slower, and thus trial effects are less pronounced (C. M. Munson, 2011, and in our data as well). Assuming batch inference substantially simplifies the model fitting process. Thus, we leave the effect of ignoring trial order as a question for future work. 
updating framework and the model fitting procedure (see Goldwater, Griffiths, \& Johnson, 2009, for a cautionary tale from word segmentation). We thus leave it as a question for future work to determine how to back off on the assumption that listeners know the underlying cluster structure.

Finally, we also include a lapse rate parameter that allows for some proportion of responses to be attributed to random guessing (e.g., because of attentional blinks). This prevents datapoints where the model predicts nearly $100 \%$ $/ \mathrm{p} /$ or $/ \mathrm{b} /$ responses from having an outside effect on the likelihood and thus the parameter estimates (see Clayards et al., 2008, for a discussion).

The posterior distributions of each of these parameters (the shared prior beliefs plus lapsing rate) were estimated by Hamiltonian Monte Carlo, using the Stan software package (Stan Development Team, 2015). We used weakly informative normal priors to prevent the sampler from getting stuck in regions of parameter space that did not affect model fit. For the expected means and variances, the priors were centered at 0 with standard deviations of 100 (making them roughly uniform over reasonable values). For the confidence parameters, the priors were centered as 0 with standard deviation of 888 (which was four times the total number of trials that listeners heard), making it essentially uniform on the whole range from completely ignoring prior beliefs to not adapting at all. The prior for the lapsing rate was uniform on $[0,1]$. We ran four chains for 1000 samples each, discarding the first 500 as burn-in for a total of 2000 samples overall. This sampler converged well and achieved good mixing (maximum $\hat{R}=1.01$; Gelman \& D. B. Rubin, 1992).

\subsubsection{Results and discussion}

The first way we evaluate this model is to ask how well it fits listeners' behavior. Figure 4.5 shows listeners' average classification functions, compared with 


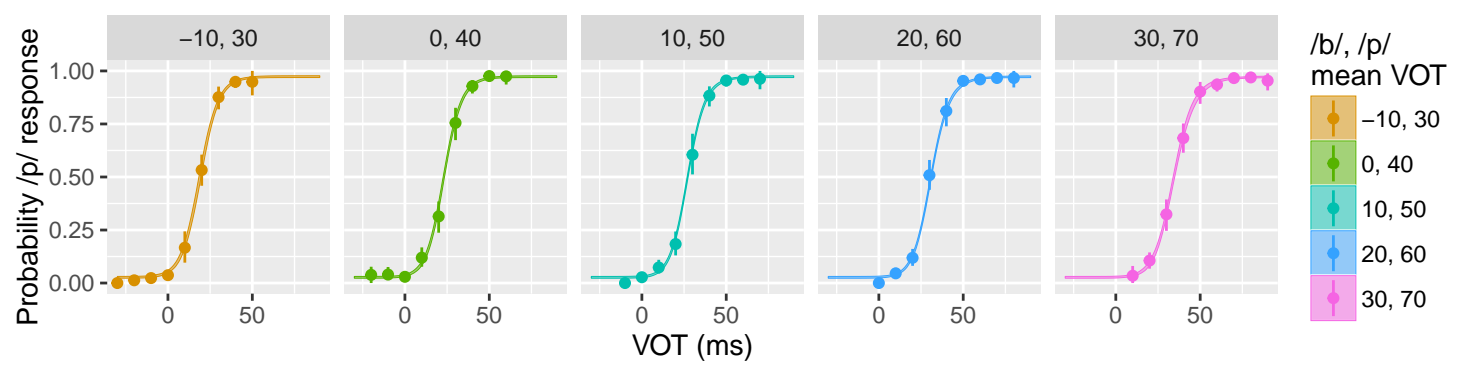

Figure 4.5: The classification functions (shaded ribbons, 95\% posterior predictive intervals) predicted by the belief updating model fit listeners' responses well (dots with lines showing bootstrapped 95\% confidence intervals).

the posterior predictive classification functions from the belief-updating model. The first thing to notice is that the model fits the data well (log-likelihood ratio vs. an intercept-only binomial null model of $1.2 e+04$, and Spearman's $\rho$ $=0.9, p<10^{-10}$ in both cases) capturing the different classification functions that result from exposure to each input distribution. This in and of itself is an interesting result: it shows that there does exist some set of prior beliefs such that the range of adaptation behavior we observed can be explained by a model where the listeners assigned to the different accent conditions all start from a common set of prior beliefs.

\section{Do inferred beliefs align with a typical talker?}

The second way to evaluate this model is based on the prior beliefs it infers listeners to have. Table 4.2 shows the posterior expectation and $95 \%$ highest posterior density intervals for each of the prior belief parameters given the adaptation data above.

Figure 4.6 shows the cue distributions corresponding to the posterior expected values of the prior expected mean and variance parameters given the behavioral data. These prior beliefs are reasonably consistent with other attempts to determine what listeners think the underlying cue distributions are (Kronrod et al., 2012), as well as the distributions produced by actual talkers 


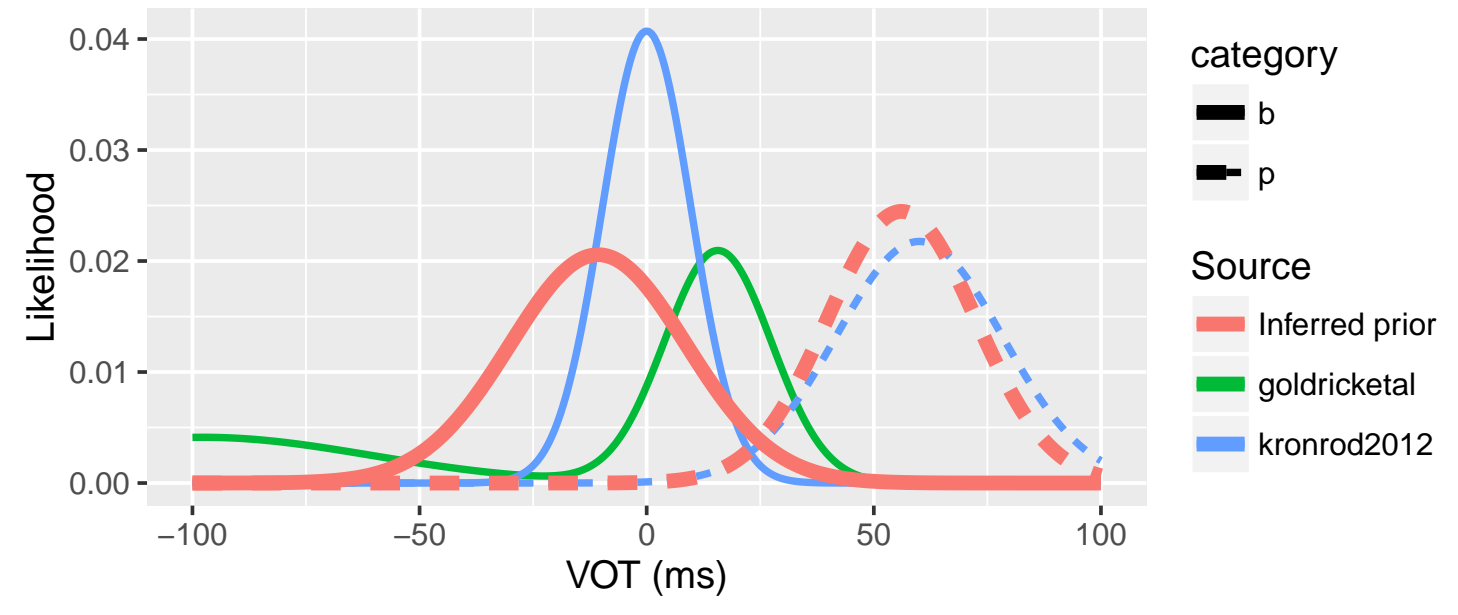

Figure 4.6: Expected cue distributions based on the prior beliefs inferred here from behavioral adaptation data. Plotted with VOT distributions measured by Kronrod et al. (2012) based on a combination of classification and discrimination behavior, and from production data by Goldrick, Vaughn, and Murphy (2013) for /b/, including pre-voicing.

(Lisker \& Abramson, 1964; Baese-Berk \& Goldrick, 2009; Goldrick, Vaughn, \& Murphy, 2013). In fact, the prior expected VOT distribution for $/ p /$ that our model inferred is almost identical to that observed by both Kronrod et al. (2012) and Lisker and Abramson (1964). The distribution for /b/ deviates from prior work, however: the mean is slightly lower $\left(\mathrm{E}\left(\mu_{0, \mathrm{~b}}\right)=-11 \mathrm{~ms}\right)$, and the standard deviation is slightly higher $\left(\mathrm{E}\left(\sigma_{0, \mathrm{~b}}\right)=19 \mathrm{~ms}\right)$.

One possible reason for this is that a substantial minority of English speakers produce pre-voiced /b/ (Lisker \& Abramson, 1964; Goldrick et al., 2013), which is characterized by a lower (negative) VOT and a higher variance (often higher even than $/ \mathrm{p} /$ ). That is, across talkers, the $/ \mathrm{b} /$ VOT distribution parameters (mean and variance) have a bimodal distribution. We assumed a single, unimodal prior distribution, and the prior beliefs we inferred to be most likely are consistent with a compromise between the two types of / b/ distributions that talkers actually produce. Figure 4.6 shows the bimodal distribution of / b VOTs observed by Goldrick et al. (2013), which has one short-lag cluster 
around 10ms VOT with low variance, and another prevoiced cluster centered around $-100 \mathrm{~ms}$ VOT, with high variance. The model-inferred /b/ distribution is a reasonable compromise, lying in between these two clusters in its mean and variance.

This possibility suggests two directions for future work. First, existing studies of voiced stop production suggest that some talkers prevoice /b/ more than others, making the distribution of /b/ means across talkers bimodal (Lisker \& Abramson, 1964; Goldrick et al., 2013). But these studies do not include enough talkers to properly assess whether this is really representative of populationlevel variability. Moreover, the only large-scale study we know of considers only non-negative VOTs (Chodroff, Godfrey, Khudanpur, \& Wilson, 2015), making it impossible to assess the extend and variability in prevoicing. Second, more modeling work is needed to test whether a multimodal prior is justified given the adaptation data, and if so, whether it would change the inference about listeners' prior expectations for $/ \mathrm{b} /$.

This also raises the question of why Kronrod et al. (2012) — who also used perception data to estimate underlying VOT distributions-did not come to a similar conclusion. One possible answer is that listeners in their experiment did not hear a range of different accents, and therefore the resulting distribution reflect their listeners' beliefs about a single talker, not about the population. Moreover, the exposure distribution in Kronrod et al. (2012) was flat over VOTs, providing little information about the underlying distributions. Given that short-lag $/ \mathrm{b} / \mathrm{s}$ appear to be more common across talkers (accounting for the majority of tokens in both Lisker \& Abramson, 1964; and Goldrick et al., 2013), the best guess for a single, particular talker's / b/ distribution would be the short-lag distribution. A second, related reason is that if a talker produces any short-lag VOTs at all, the prevoiced cluster will not have a substantial effect on the optimal classification boundary, since it will have lower likelihood than 
either the short-lag or / $\mathrm{p} /$ distributions for VOTs near the boundary.

Table 4.2: Expected values and 95\% highest posterior density intervals for the prior parameters, given the adaptation data.

\begin{tabular}{lrll}
\hline Parameter & Expected & $95 \%$ HPD Int. & Units \\
\hline$\kappa_{0}$ & 243 & $163-495$ & observations \\
$\mu_{0, \mathrm{~b}}$ & -11 & $-28-3$ & ms VOT \\
$\mu_{0, \mathrm{p}}$ & 56 & $44-72$ & ms VOT \\
$v_{0}$ & 772 & $493-1180$ & observations \\
$\sigma_{0, \mathrm{~b}}$ & 19 & $16-23$ & ms VOT \\
$\sigma_{0, \mathrm{p}}$ & 16 & $14-20$ & ms VOT \\
\hline
\end{tabular}

\section{How confident are listeners in their prior beliefs?}

Qualitatively, the prior parameters show that listeners have high confidence in their prior expectations about the mean and variance of $/ \mathrm{b} /$ and /p/VOT distributions. The model-inferred confidence in category variances is $\mathrm{E}\left(v_{0}\right)=772$, which is is higher than the inferred confidence about means $\left(\mathrm{E}\left(\kappa_{0}\right)=243\right)$. Both of these can be interpreted as pseudo-counts, and both are larger than the number of trials that listeners heard in the experiment (222). This means that as far as the belief-updating model is concerned, listeners' beliefs about the exposure talker's /b/ and /p/ distributions reflected their prior beliefs as much as (in the case of the means) or more than (for the variances) the distributions they experienced in the experiment. This is consistent with the qualitative finding that listeners' category boundaries are intermediate between the boundaries corresponding to a typical talker and the experimental exposure talker. In previous work modeling adaptation to a $/ \mathrm{b} /-/ \mathrm{d} /$ contrast, we found that listeners 
had less confidence in their prior beliefs than we found here. As we discuss below, this apparent discrepancy is in fact predicted by the ideal adapter, at least qualitatively, based on patterns of between-talker variability.

\section{Do inferred beliefs accurately reflect cross-talker variability?}

Lastly, we can ask whether the model-inferred beliefs match the actual level of between-talker variability in VOT distributions.

In this model, the prior confidence parameters control how much VOT distributions are expected to vary across talkers. Specifically, the variance in category means is, under the form of prior we used, equal to the expected withincategory variance divided by the pseudocount corresponding to the confidence in the mean, $\operatorname{Var}\left({ }^{-}\right)=œ_{0, c}^{2} / \sim_{0}$ (Murphy, 2007). Numerically, this corresponds to cross-talker variability in category means on the order of a standard deviation of 1-2ms VOT. This underestimates the actual level of variability across talkers. Based on production data from both read (Baese-Berk \& Goldrick, 2009; Goldrick et al., 2013; Lev-Ari \& Peperkamp, 2013) and conversational speech (extracted from the Buckeye corpus, Wedel in prep), actual cross-talker variability is on the order of a standard deviation of $3-4 \mathrm{~ms}$ VOT for (short-lag) /b/ and $10-15 \mathrm{~ms}$ VOT for $/ \mathrm{p} /$.

This is likely due to the particular mathematical form we assumed listeners' prior beliefs to have. The conjugate Normal- $\chi^{-2}$ prior is mathematically convenient (in that it allows Bayesian belief updating to be expressed analytically in closed form), but it makes the assumption that cross-talker variance is proportional to within-talker variance (Gelman, Carlin, et al., 2003). This assumption may not be warranted in reality, and it's a question for future modeling work to explore the feasibility and consequences of relaxing this assumption. 


\subsection{Experiment 2}

The results of Experiment 1 show that listeners' adaptation to an unfamiliar talker is constrained. Such constraints are predicted by the ideal adapter framework, which says that listeners use their knowledge of how VOT distributions vary across talkers to guide their adaptation to an unfamiliar talker. Our modeling results provide further evidence for this explanation, and show that the constraints are consistent with belief updating starting from a single set of prior beliefs about cross-talker variability in VOT distributions.

In Experiment 2, we provide a further, more specific test of the ideal adapter explanation. There are many ways that cue distributions can vary, and in Experiment 1 we manipulated just one of them, shifting a single bimodal VOT distribution up or down but leaving the overall shape the same. Experiment 2 goes one step further, shifting the means of the $/ \mathrm{b} /$ and $/ \mathrm{p} /$ clusters semiindependently. This results in bimodal distributions that differ both in the overall mean VOT, and in the separation of the /b/ and /p/ clusters (Figure 4.7).

The main goal of this experiment is to test the predictive power of the model and prior beliefs it inferred listeners to have based on the data from Experiment 1. Above we showed that belief updating-starting from these inferred prior beliefs-provides an excellent fit to listeners' adaptation behavior across conditions in Experiment 1. However, the real utility of a model is its ability to predict behavior in new situations. The qualitatively different set of input distributions we use in Experiment 2 provides a stringent test of the predictive utility of this framework.

The particular set of distributions we chose was informed by how the model fit the data from Experiment 1. The posterior distributions of the model parameters given the data were not independent: the prior expected mean VOTs for /b/ and /p/ were inversely correlated, which means that the model fit was 
more uncertain about the distance between the two category means than the midpoint between them. This reflects the fact that the exposure distributions in Experiment 1 all had the same separation between the cluster means $(40 \mathrm{~ms}$ VOT). By varying the separation between the clusters we test how much the uncertainty inherent in the structure of the first experiment affects the predictive power of the model.

Another, secondary goal is to test how listeners adapt to large, negative VOTs. The inferred prior beliefs about the /b/ VOT distribution of a typical talker have higher variance than the short-lag distribution generally considered to be typical of American English, but are consistent with a compromise between short-lag and prevoiced distributions. One way to determine whether listeners consider prevoiced and short-lag VOT distributions are a single cluster or two different clusters is to directly compare adaptation to short-lag and prevoiced distributions, which informs the specific choice of distributions we used in this second experiment. In the short term, this allows us to assess how well the model's inferred prior beliefs generalize to adaptation to prevoiced distributions. In the long term, it provides data to directly compare the current model (that treats $/ \mathrm{b} /$ as a single VOT cluster) to a model that treats $/ \mathrm{b} /$ as a mixture of short-lag and prevoiced clusters. Such a model is a conceptually straightforward — but technically difficult—extension of the current model. That is, Experiment 2 is aimed at facilitating future modeling work that can provide insights into the sort of phonetic category representations that listeners might be using to process speech. By focusing on the different possible sorts of distributional representations, this has the potential to go beyond the computational principles like distributional learning that modeling work in this framework has largely focused on. 


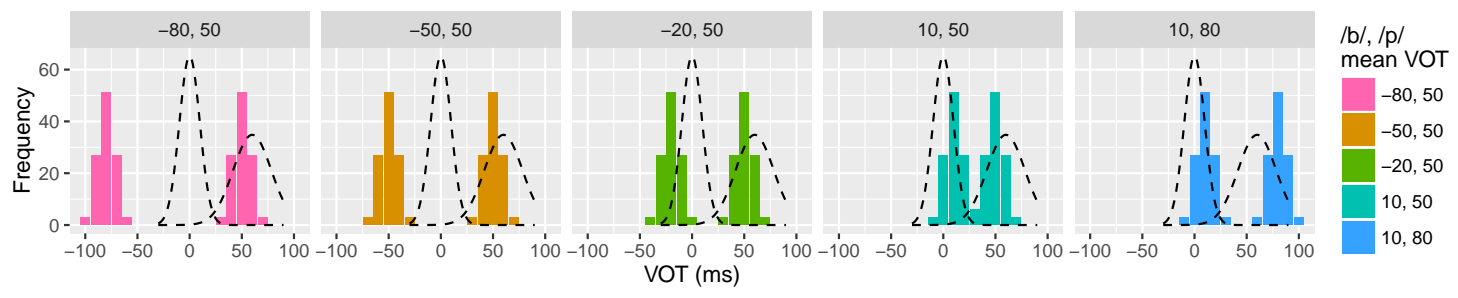

Figure 4.7: In Experiment 2, each subject heard a talker that produced one of these five VOT distributions. The variance of each category was constant across conditions, but the means varied semi-independently.

\subsubsection{Methods}

As in Experiment 1, listeners performed a distributional learning task, hearing a talker who produced one of five different bimodal VOT distributions. Across these distributions, we varied $/ \mathrm{b} /$ and $/ \mathrm{p} /$ mean VOT semi-independently (Figure 4.7). These distributions cover a wider range of implied category boundaries (from -15 to $45 \mathrm{~ms}$ VOT) than Experiment 1 (10 to $50 \mathrm{~ms}$ ), especially on the lower end. They also varied in the distance between cluster means (from 40 to $130 \mathrm{~ms}$ VOT), unlike Experiment 1 which maintained a constant separation between $/ \mathrm{b} /$ and $/ \mathrm{p} /$ mean VOT of $40 \mathrm{~ms}$. Note that the condition with /b/ mean of $10 \mathrm{~ms}$ and /p/ mean of 50ms VOT is the same as in Experiment 1. This condition was included to provide a way of comparing with Experiment 1, since the design of Experiment 2 required a change in procedure (which we discuss below).

\section{Subjects}

We recruited 124 subjects via Amazon's Mechanical Turk. Subjects were paid $\$ 2.50$ for participation. The task took subjects about 25 minutes to complete (because of the additional post-test, discussed next). As in Experiment 1, we excluded subjects $(n=5)$ who failed to classify words with unambiguous VOTs reliably during exposure (not post-test, see below). After exclusion, 119 sub- 
jects remained for analysis (no subjects had previously participated in one of our VOT studies). Subjects were evenly distributed across conditions (range of 23 to 25 per condition).

\section{Procedure: assessing category boundaries}

The procedure was identical to Experiment 1, with one exception. After completing the 222 trial exposure phase as in Experiment 1, listeners completed a test phase in order to assess their category boundaries. This phase consisted of 70 additional trials with VOTs evenly distributed from -10 to $50 \mathrm{~ms}$ in $10 \mathrm{~ms}$ steps. This additional phase was necessary because of the large separation between $/ \mathrm{b} /$ and $/ \mathrm{p} /$ clusters in the exposure distributions meant that in most conditions there were no trials with VOTs anywhere near the predicted (or typical) boundaries.

Critically, listeners were not told about the change from exposure to test phase: the procedures were identical, and there was no break in between. Besides the change in VOT distributions, there was no way for listeners to tell that they had entered the test phase. Of course, if listeners are actually (as we hypothesize) learning these distributions, their behavior may well change as they proceed through the test phase, gradually erasing any effect of the differences in the exposure distributions. Thus, when analyzing data from the test phase, whenever possible we limit ourselves to the early parts of the test phase, when listeners behavior should be minimally affected by the change in distributions.

As in Experiment 1, we evaluated listeners adaptation by their classification functions, which we estimated by fitting a logistic GLM. In addition to VOT, we included a predictor for trial in order to account for any (un-)learning effects that might happen during post-test. In order to visualize the fitted classification functions and to estimate listeners' category boundaries, we used the classifi- 
cation function estimates from the trial halfway through the first third of the post-test. ${ }^{6}$

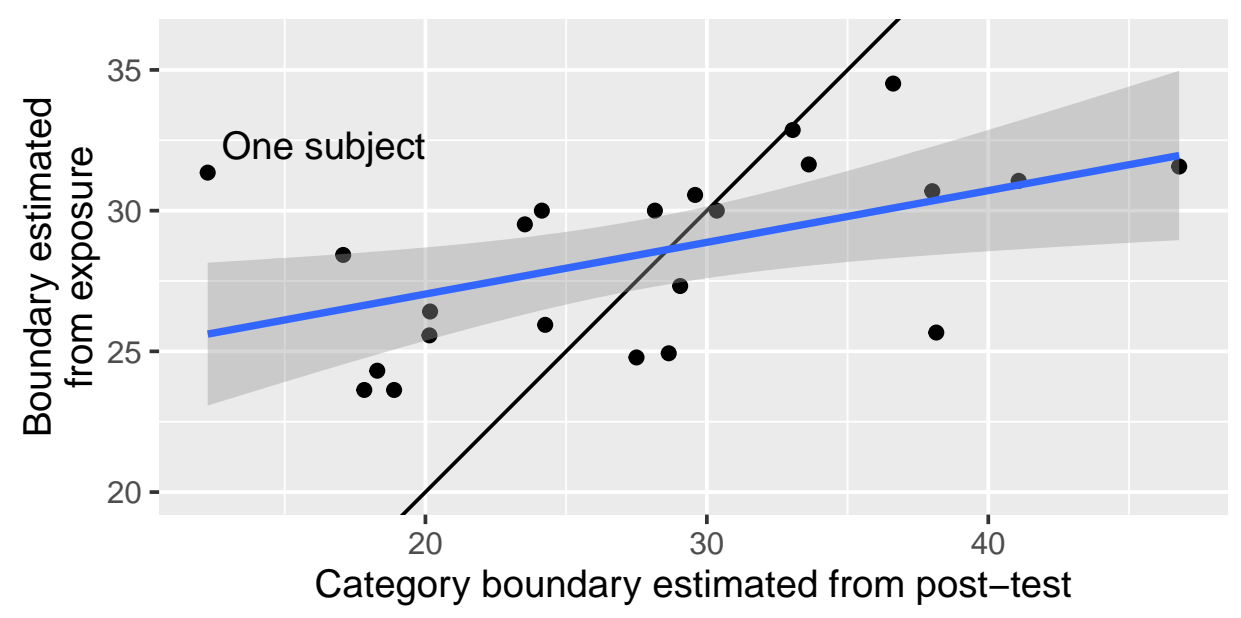

Figure 4.8: Category boundaries estimated during post-test are correlated with estimates from exposure (in the 10, 50 condition where such an estimate is possible), but more variable. Blue line shows best linear fit, and black line shows where the two estimates are equal.

We validated this procedure in the $10 \mathrm{~ms}, 50 \mathrm{~ms}$ mean / $\mathrm{b} /$ VOT distribution condition, which is the same as in Experiment 1. We first obtained an estimate of each listener's category boundary based on their responses during the exposure phase, as in Experiment 1. We then obtained a second estimate for these same subjects using the novel procedure, based on the post-test phase. This allowed us to compare the method of assessing listener's category boundaries from Experiment 1 (using exposure trials themselves) with the following posttest trials within the same listeners. Figure 4.8 shows that the post-test estimates are more variable than the exposure estimates (standard deviations across subjects of 8.75 and 3.21, respectively) but correlated with (Pearson's $R=0.5,95 \%$

\footnotetext{
${ }^{6}$ The predictions from a regression model are more uncertain and more driven by noise at the extreme ranges of continuous predictors, like trial number. Thus we use the point one-sixth into the post-test to estimate classification functions, rather than the very beginning, because it is close to the beginning of the post test, but not so close that the predictions are substantially affected by the extra instability of the predictions that come at the edge of a continuous predictors range.
} 
$\mathrm{CI}=[0.11,0.76], p<0.05)$. Critically, they are not biased, differing only by $0.75 \mathrm{~ms}$ VOT on average ( $95 \% \mathrm{CI}$ of $-2.4-4.0 \mathrm{~ms}$ VOT). This means we can compare the boundary estimates from Experiments 1 and 2, despite the differences between the procedures.

The inclusion of this condition in both experiments also allows us to replicate the results of Experiment 1 using a different method (Figure 4.10, unshaded distribution). This allows us to test how well the prior beliefs inferred from Experiment 1 can predict adaptation to the same exposure distributions by a different set of subjects, using a slightly different procedure (see below, and Figure 4.11).

\subsubsection{Results}

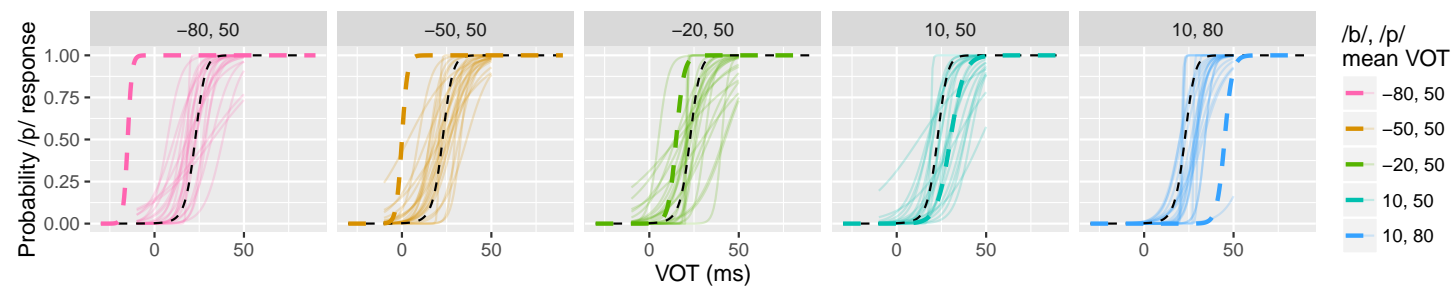

Figure 4.9: Listeners' categorization functions in Experiment 2 (during posttest) reflect partial adaptation to the exposure talker, especially for more extreme conditions extreme distributions. Adaptation was even less complete than in Experiment 1. Classification functions are estimated with a logistic GLM including trial; curves show predictions for the beginning of the post-test phase to minimize impact of unlearning during test.

As in Experiment 1, listeners adapted to the different input distributions, but in a constrained way. On average, listeners' classification functions were intermediate between the boundaries based on a typical talker's distributions and the exposure talker's distributions. In the one condition that was shared with Experiment 1 (mean VOT of $10 \mathrm{~ms}$ for $/ \mathrm{b} /, 50 \mathrm{~ms}$ for $/ \mathrm{p} /$ ), listeners in Ex- 


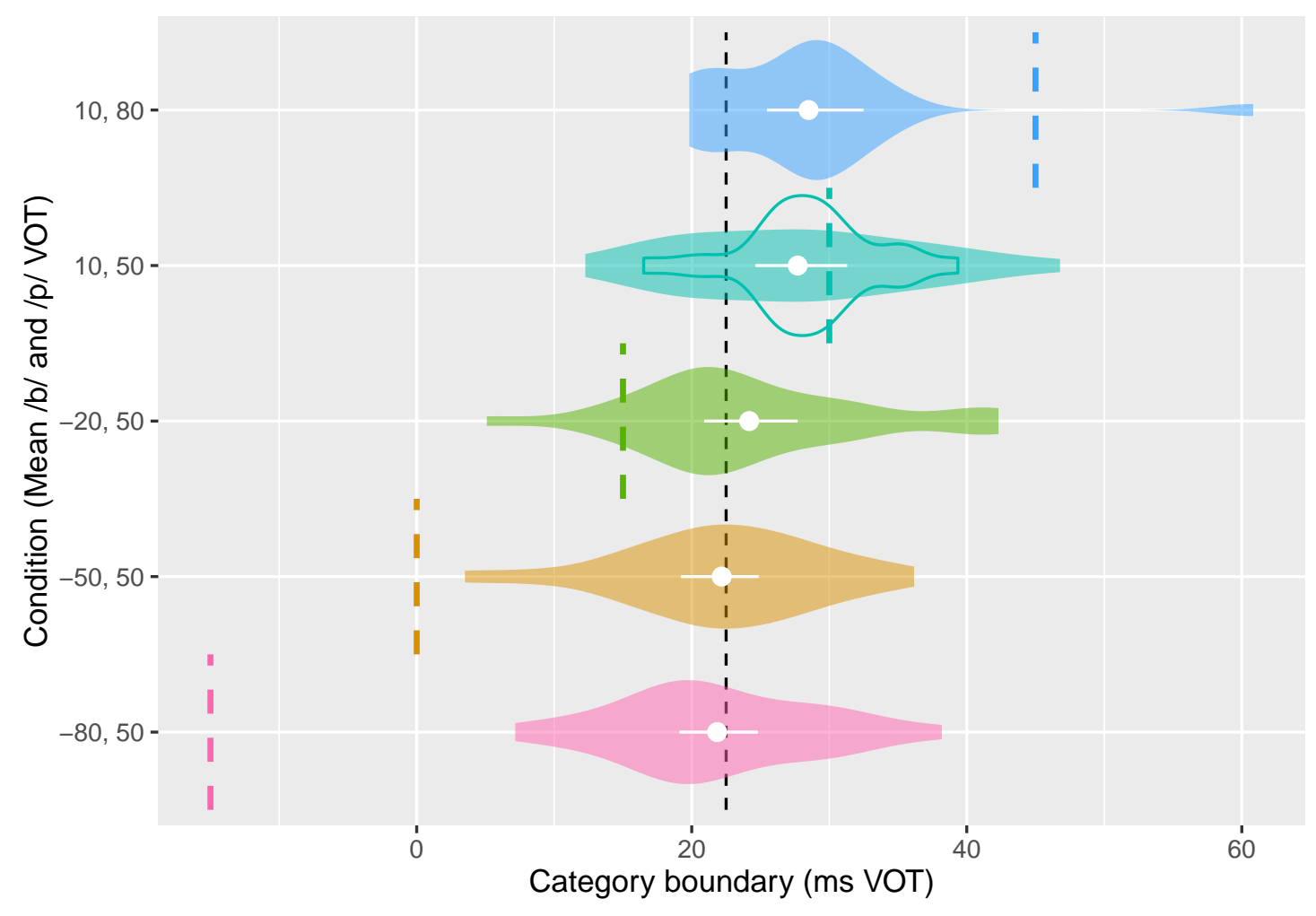

Figure 4.10: The distribution of listeners' individual category boundaries in Experiment 2 reflects partial adaptation to the exposure talker's VOT distributions. Extreme shifts have minimal additional effect on listeners' boundaries, corroborating the strong prior biases observed in Experiment 1. The un-filled distribution shows the boundaries from listeners in the corresponding $10 \mathrm{~ms}$ $\mathrm{VOT} / \mathrm{b} /$ mean condition from Experiment 1 . These conditions had the same exposure distributions, but differed in whether category boundaries were estimated based on the exposure (Experiment 1) or post-test data (Experiment 2). 
periment 2 adapted just as much as those in Experiment 1, but the estimates of their boundaries were more variable (Figure 4.10, empty vs. filled distribution).

\section{Evidence for separate prevoiced cluster?}

Listeners showed very little sensitivity to increasingly large negative mean /b/ VOTs: the average category boundaries were very similar in the $-20,-50$, and $-80 \mathrm{~ms} / \mathrm{b} / \mathrm{mean}$ conditions. On average these were comparable to the $0 \mathrm{~ms}$ /b/, 40ms /p/ mean condition from Experiment 1, which had a higher /b/ mean but lower $/ \mathrm{p} /$ mean. This suggests that listeners are more ready to adapt to variation in $/ \mathrm{p} /$ than in $/ \mathrm{b} /$. Nevertheless, listeners in the $10 \mathrm{~ms} / \mathrm{b} /, 80 \mathrm{~ms}$ $/ \mathrm{p} /$ still did not fully adapt to the exposure distribution, producing category boundaries that were not significantly different from those in the $10 \mathrm{~ms} / \mathrm{b} /$, $50 \mathrm{~ms} / \mathrm{p} /$ condition (bootstrapped $p=0.73$ ).

One interpretation of this lack of sensitivity to large negative / b/ mean VOTs is that listeners treat $/ \mathrm{b} / \mathrm{as}$ being a mixture of a short-lag cluster (with a mean around $0 \mathrm{~ms}$ VOT) and a prevoiced cluster (with a large negative mean VOT). Most American English talkers produce at least some short-lag VOTs, even if they produce mostly prevoiced. Goldrick et al. (2013) found that, even the talker who prevoiced the most still produced short-lag VOTs $8 \%$ of the time. Thus, even for a talker who produces mostly prevoiced $/ \mathrm{b} / \mathrm{s}$, a listener needs to be ready for some short-lag $/ \mathrm{b} / \mathrm{s}$ as well. Because the short-lag distribution is closer to the $/ \mathrm{p} /$ distribution, it is the one-rather than the prevoiced cluster-that determines where an ideal listener's category boundary will fall. In conditions where the exposure distributions do not provide very much (if any) information about a talker's short-lag distribution, we would thus expect listeners' category boundaries to be unchanged from a typical talker. This is in fact exactly what we observe in the Experiment 2 conditions with large negative /b/ mean VOTs. 
There are other possible explanations for the lack of large shifts in these conditions. For instance, even if listeners treat /b/ as a single unimodal cluster, the negative VOT distributions we used are still extreme relative to the range of /b/ mean VOTs across talkers. In the next section, we address this possibility, by evaluating how well the prior beliefs inferred from Experiment 1-which assume a single / $\mathrm{b} /$ cluster-predict the pattern of adaptation here.

\section{Predicted adaptation from inferred priors}

Next, we ask how well the prior beliefs inferred on the basis of Experiment 1 can predict the pattern of adaptation across exposure distributions we observed in Experiment 2. Figure 4.11 shows the predicted classification functions (95\% posterior predictive intervals) for the exposure conditions in Experiment 2, using the same parameters that were fit to Experiment 1 (and visualized in Figure 4.5).

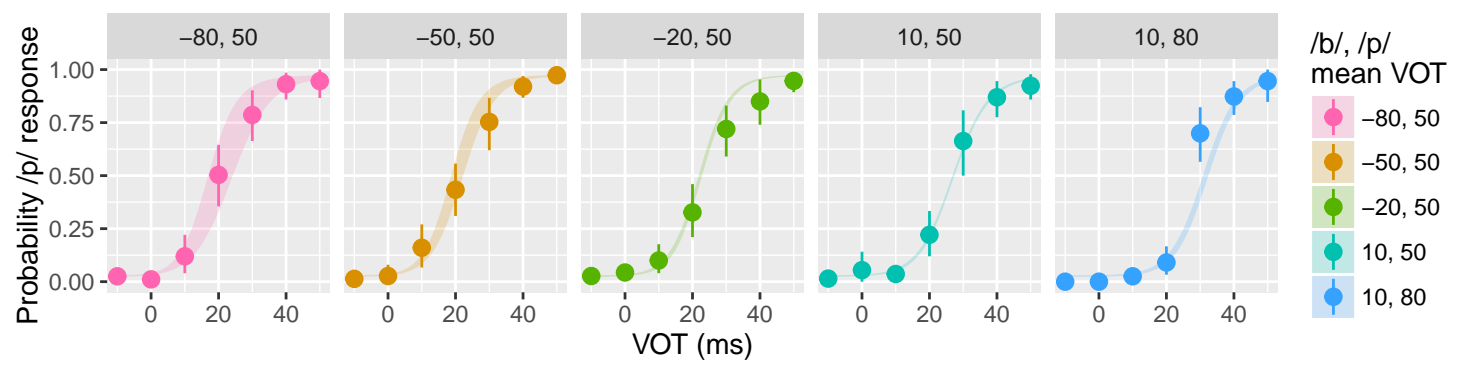

Figure 4.11: The prior beliefs inferred from Experiment 1 predict how much listeners adapt to each of the input distributions of Experiment 2. Shaded regions show the $95 \%$ posterior predictive intervals for the belief updating model, based on prior beliefs inferred from Experiment 1 and exposure distributions from Experiment 2. Dots and errorbars show mean and $95 \%$ bootstrapped CIs for the mean probability of $/ \mathrm{p} /$ response over subjects. Note the additional uncertainty in predictions relative to the posterior predictive distribution given Experiment 1's conditions (Figure 4.5).

Overall, the predicted classification functions line up reasonably well with listeners' actual category boundaries at test (Figure 4.11; Spearman's $\rho=0.83$, 
log-likelihood ratio with intercept-only null model of $986.80^{\prime}$, both $p<10^{-10}$ ). In particular, the excellent fit to the $10 \mathrm{~ms} / \mathrm{b} /, 50 \mathrm{~ms} / \mathrm{p} /$ mean condition shows that the prior beliefs inferred in Experiment 1 effectively predict adaptation to the same exposure distributions in Experiment 2, despite the differences in test procedures.

Moreover, the category boundaries listeners actually produce align well with the boundaries predicted based on the inferred prior beliefs from Experiment 1 . The average difference between predicted and observed category boundary is small (average absolute deviation across conditions o2.28)ms VOT). These differences are not significantly higher or lower than zero in any condition (all bootstrapped $\$ p>0.1 \$$, with the exception of the $-80 \mathrm{~ms}, 50 \mathrm{~ms}$ and the $10 \mathrm{~ms}, 80 \mathrm{~ms}$ conditions where $\$ 0.057) \$$ and 0.067$)^{\prime} \$$, respectively)

The model predictions become less accurate as the test phase progresses (likelihood ratios vs. null model for the first, second, and final third of the posttest phase 0987,974 , and 963)' $)$. This suggests that listeners may be unlearning the distributions encountered in the test phase. Such unlearning is consistent with other work that shows that phonetic adaptation (recalibration) can be undone by prolonged testing (Kraljic \& Samuel, 2005; Vroomen, van Linden, Keetels, et al., 2004; and specifically for the case of a flat test distribution, Liu \& Jaeger, 2016).

\subsubsection{Discussion}

The results of Experiment 2 provide further evidence that VOT distributional learning is constrained. Using more extreme distribution, we found even larger discrepancies between the category boundaries implied by the input distributions and listeners' actual classifications than in Experiment 1. In fact, it ap- 
pears that moving the $/ \mathrm{b} /$ mean VOT from -20 to -50 and even -80 has little additional effect on the category boundary, even though each of these changes would move the boundary by $-15 \mathrm{~ms}$ VOT each (if the prior was completely ignored).

While these constraints might appear to be even more extreme than we observed in Experiment 1, they are in fact predicted from the results of Experiment 1 by our inverted belief-updating model. This model treats adaptation to the exposure talker as a process of optimal (or near-optimal) distributional learning, which involves integrating current experience with prior expectations. The predictions of this model are in generally good agreement with listeners' behavior in Experiment 2, despite the fact that the input distributions differ markedly from the Experiment 1 data used to fit the model.

\subsection{General discussion}

In two experiments, we have evaluated how well listeners can adapt to different pseudo-'accents' by learning distributions of VOTs. Our results confirm previous findings that listeners can adapt to novel accents like these via distributional learning (Clayards et al., 2008; C. M. Munson, 2011). We go beyond previous work by systematically evaluating adaptation performance on a range of different distributions. Our results show that listeners do not adapt as well to extreme distributions that deviate substantially from what it typical of American English. This goes to show that while distributional learning is, in principle, a very powerful general-purpose approach to dealing with talker variability, it is constrained —or, we have argued, guided by prior experience-in actual practice. 


\subsubsection{Constraints on distributional learning}

Where do these constraints come from? A central tenet of the ideal adapter framework (Kleinschmidt \& Jaeger, 2015) is that listeners can—and shouldbenefit from structure in how talkers vary in the cue distributions they produce. Even if all a listener knows is that a talker is speaking American English, their prior experience with other speakers of American English provides a lot of information about the cue distributions that they should expect, which makes the process of inferring that particular talkers' distributions more efficient. At the same time, this view predicts that listeners will pay a price when they encounter a talker whose distributions fall outside the range they expect (such as when encountering novel accents, languages, or-to a milder extent-the present experiments). In order to get as much benefit from the head start provided by prior expectations while avoiding the cost of over-confidence, listeners should match their prior expectations to the actual variability across talkers in the world.

Our modeling results show that this explanation is at least consistent with the pattern of constraints that we observed behaviorally. The range of adaptation behavior across different distributions in Experiment 1 can be fit very well by a model that assumes that listeners update their beliefs based on the distributions they experience, starting from a prior expectation about what a typical talker sounds like and how much talkers vary. These prior expectations also effectively predict how well listeners will adapt to the substantially different exposure distributions in Experiment 2. Moreover, the expectations that we infer listeners to have for an unfamiliar talker match the actual cue distributions produced by American English speakers reasonably well. (with the caveat that our model makes potentially problematic assumptions about the structure of categories within and across talkers, which we discuss below). 


\subsubsection{What is the source of constraints on distributional learn- ing?}

We have proposed that the constraints on distributional learning we observed in Experiments 1 and 2 are learned by listeners from their experience with how phonetic cue distributions vary across talkers. There are, of course, other possible sources for these constraints. For instance, the basic nature of the mammalian auditory system may constrain the distributions that are actually psychoacoustically distinguishable. Failure to adapt to particular distributions may then simply be a side effect of these distributions being difficult to perceive because of non-linearities in the underlying psychoacoustic dimensions (which we model as linear in VOT). This is a plausible explanation for VOT in particular. Cross-linguistically, category boundaries occur at a small number of VOT values (Lisker \& Abramson, 1964). ${ }^{7}$ Moreover, non-human animals also categorize voiced and voiceless stops along these boundaries as well (Kuhl \& J. D. Miller, 1975). Moreover, the typical boundary between (short-lag) voiced and voiceless word initial stops is approximately the same as the temporal asynchrony required for neural responses to separately time lock to two events, and thus identify the release burst and onset of voicing as distinct acoustic events (Steinschneider, Schroeder, Arezzo, \& Vaughan, 1994).

Another possibility is that listeners' expectations are driven by their own productions, and that any regularity between these comes from acquisition or innate anatomical constraints (e.g., Moulin-Frier, Diard, Schwartz, \& Bessière, 2015). For instance there are limits on the VOTs that can be produced by the human vocal tract, although this would not explain our results here because the actual VOTs of our stimuli are all well within the range of what is phys-

\footnotetext{
${ }^{7}$ Such cross-linguistic regularities must, however, be interpreted with caution, since they may be due to shared ancestry of languages or language contact (e.g., Jaeger, Graff, Croft, \& Pontillo, 2011), rather than reflecting innate constraints.
} 
ically possible (and actually attested; Baese-Berk \& Goldrick, 2009; Goldrick et al., 2013). Other, intermediate possibilities exist, too. For instance, listeners' expectations may be based on their own productions, but their confidence based on the level of cross-talker variability they have experienced.

These alternative explanations may be plausible in the case of voicing, in large part because the cue distributions are generally consistent across talkers (Allen, J. L. Miller, \& DeSteno, 2003). However, many contrasts-especially those that are distinguished by spectral cues—do vary substantially across talkers, and moreover show substantial stylistic variability that cannot be normalized away (McMurray \& Jongman, 2011; Jongman et al., 2000; Hagiwara, 1997; Hillenbrand et al., 1995; Clopper, Pisoni, \& de Jong, 2005; Newman et al., 2001).

Testing for constraints on adaptation for these other, more variable contrasts would help resolve these different possible explanations. For instance, if listeners are more constrained than would be expected based on cross-talker variation, it would suggest that the constraints come from something like their own productions, since levels of within talker variability are similar across contrasts (Newman et al., 2001; Allen, J. L. Miller, \& DeSteno, 2003). The presence of structure in cross-talker variation also provides a means to test more specific predictions of the ideal adapter. In particular, the ideal adapter predicts that when talkers cluster into groups, listeners' distributional learning should be informed (and hence constrained) by their experience with within-group variability, not only population-level variability. For instance, if listeners pick up on the fact that male and female talkers systematically differ in their vowel formant distributions, then knowing that a talker is male or female should lead to more constrained adaptation than if they do not know the gender of the talker (all else being equal, including other cues to gender). 


\subsubsection{Measuring listeners' subjective beliefs about unfamiliar talkers}

Our other major finding is that it is possible to infer what cue distributions listeners expect from an unfamiliar talker, based solely on listeners adaptation behavior (as measured by phonetic classification). The actual beliefs that our model infers listeners to have provide a good fit to this adaptation behavior, can predict adaptation for distributions that were not used to train the model, and align reasonably well with distributions that talkers actually produce. The combination of presenting listeners with a parametrically manipulated range of distributions and analyzing the resulting changes in phonetic classification with a belief updating model provides a potentially powerful tool for measuring listeners' expectations. This is important because these expectations are an essential part of how listeners manage to adapt to efficiently to talker variability, but are difficult to measure, being implicit and subjective. The subjective nature of these expectations is particularly important, because they may deviate in substantial ways from the actual nature of cross-talker variation. For instance, arbitrary aspects of linguistic variation can become "enregistered", where listeners consider particular variants stereotypical of social groups, whether they are in fact differentially associated with them or not (see, e.g., Eckert, 1989; Eckert, 2012b; Podesva, Roberts, \& Campbell-Kibler, 2001; Niedzielski, 1999; Levon, 2014).

This tool nevertheless has limitations. Most importantly, the prior beliefs a model can infer are fundamentally bound by the assumptions the model makes about that nature of those beliefs and how they are updated. If the structure of the world does not align with what is assumed by the model, the models' inferences may be misleading in subtle ways, even if the predictive accuracy of the model is good. For instance, we assumed that each phonetic category is a sin- 
gle normal distribution, but in reality the way people actually produce /b/ appears to be a mixture of two distinct clusters. Even so, our model effectively predicted adaptation to different distributions, even with negative VOTs where the structure of the /b/ category seems like it should make a difference. The beliefs that the model infers listeners to have for $/ \mathrm{b} /$, though, do not look like distributions of VOTs that talkers actually produce for $/ \mathrm{b} /$. Instead, they look like a good approximation of the true distribution using only a single cluster, with a mean and variance that is intermediate between the two clusters talkers actually produce. It would be premature to conclude that listeners actually believe a typical talker would produce this distribution. Rather, this discrepancy highlights areas where more data is needed. That is, a model need not be true for it to be useful, as long as it's understood as a means for interpreting and guiding empirical work and not a source of ground truth.

We also assumed that listeners prior beliefs can be modeled using a particu-

lar form, the Normal- $\chi^{-2}$ distribution. This is mathematically convenient, but makes the assumption that listeners' uncertainty about category means across talkers is proportional to their beliefs about variance within a talker. This is unlikely to be an accurate reflection of actual patterns of talker variability, but it is not immediately obvious what the consequences of this assumption are. It may explain the discrepancy between the amount of talker variability that actually occurs and the level predicted by the model's inferred beliefs, but this remains to be seen.

\subsubsection{What makes listeners confident in their prior beliefs?}

Our modeling results suggest that listeners have high confidence in their prior expectations about the VOT distributions of $/ \mathrm{b} /$ and $/ \mathrm{p} /$, acting as if they had already observed around 200800 samples from each category (for the category 
means and variances, respectively) from the unfamiliar talker they encountered in our experiment. This is consistent with the qualitative findings in Experiments 1 and 2 that distributional learning is substantially constrained.

However, at first blush, this conflicts with our previous modeling work on adaptation to another phonetic contrast, /b/-/d/, which found confidence values that were one or two orders of magnitude smaller than those inferred here (Kleinschmidt \& Jaeger, 2015). This contrast is cued by spectral cues (formant frequency transitions) which generally vary substantially across talkers (e.g., Peterson \& Barney, 1952). The acoustic cues to the $/ \mathrm{b} /-/ \mathrm{p} /$ contrast used in the current study do not show as much variability across talkers (e.g., Allen, J. L. Miller, \& DeSteno, 2003; Chodroff et al., 2015). When there is little variability across talkers, past experience with other talkers' VOT distributions is highly informative about the distributions that an unfamiliar talker will produce, requiring less adaptation. Likewise, when there is more variability across talkers, listeners need to rely more on the current talker's cue distributions and less on their prior experience. Thus, the apparent discrepancy between the confidence that listeners place in their prior beliefs in the current study and in Kleinschmidt and Jaeger (2015) is actually in line with an ideal adapter which combines prior beliefs with current experience weighted according to confidence.

This idea finds further empirical support in Kraljic and Samuel (2007), who found that after the same amount of exposure, listeners recalibrate a /d/-/t/ contrast (analogous to the $/ \mathrm{b} /-/ \mathrm{p} /$ contrast used here) much less than an $/ \mathrm{s} /$ $/ \mathrm{J} /$ contrast (where the latter exhibits larger variability across talkers; e.g., Newman et al., 2001). This leads to the following clear prediction for future work: Listeners should adapt more flexibly to phonetic contrasts where there is more talker variability. At present this prediction is qualitative. In order to make it more precise, we need better measurements of the actual extent of talker vari- 
ability in different contrasts. Chapter 5 provides a first step in this direction. Based on what we know about talker variability at the moment, we specifically predict that distributional learning for vowels and fricatives should be more flexible (i.e., best fit with lower confidence parameters) than for stop voicing.

\subsection{Conclusion}

In order to cope with cross-talker variability, the speech perception system needs to be highly flexible. At the same time, a system that is completely flexible, adapting from scratch every time, would be ineffective. One solution to this dilemma is to leverage prior experience with other talkers in order to provide an informative starting point when adapting to an unfamiliar talker. This is what rational accounts predict (i.e., the ideal adapter, Kleinschmidt \& Jaeger, 2015). More specifically, these accounts predict that prior experience constrains (or guides) learning and adaptation to an unfamiliar talker's accent. Even if all the listener knows about the talker is that they are speaking English, they can still benefit from prior experience with other speakers of English to provide an informative head start for adaptation. In this view, prior experience can only be helpful to the extent that it rules out some possible accents (cue distributions). If a listener encounters a talker whose accent (cue distributions) lies outside the range that they think is a priori plausible based on their prior experience, they will have a hard time adapting to that talker (cf. Pajak, Fine, et al. (2016) for a discussion of second-language learning as an extreme example from this perspective).

We found that listeners' behavior in two distributional learning experiments is constrained and guided in exactly this way. Specifically, we found that listeners' classification of a novel talker's VOT continuum reflects a compromise between what would be expected for the VOT distributions produced by a typ- 
ical talker and the exposure talker. More specifically, the range of adaptation behavior observed across the various accents that listeners heard can be captured by a belief-updating model with a single set of prior expectations that are updated based on experience with the exposure talker. Moreover, these inferred beliefs effectively predict adaptation to different distributions.

The modeling framework we use has the additional advantage of allowing us to infer what cue distributions listeners believe an unfamiliar talker will produce. This provides a potentially powerful-and heretofore missing-tool for probing listeners' prior expectations, based only on comprehension data. These beliefs reflect what listeners have learned about the variability they can expect across talkers, and probing how this internal model is related to the actual variability across talkers (measured via speech production data) is an important next step in advancing our understanding of robust speech perception.

More generally, prior knowledge is increasingly understood to play in important role in a number of perceptual and memory domains (e.g, Brady \& Tenenbaum, 2013; Froyen, Feldman, \& Singh, 2015; Orhan \& Jacobs, 2011). Distributional learning provides an approach to probing prior expectations about the statistics of the sensory world, which, as in speech perception, are critical to effectively coping with non-stationarity in sensory statistics. 


\section{Quantifying the role of socio-indexical structure in}

\section{talker variation}

Variability is one of the defining features of speech. There are two broad traditions in the study of speech, with very different approaches to the role of variability in speech. On the one hand, for the cognitive/psycholinguistic tradition, variability is one of the central problems of speech perception, a challenge that listeners must cope with. In this view, variability is such a severe problem that it is traditionally referred to only indirectly, as the "lack of invariance" (Liberman et al., 1967). The sociolinguistic tradition, on the other hand, views variability as a rich source of social information. The particular variety of language that you speak says a lot about who you are as a person, and sociolinguistics takes this sort of variation as one of its primary objects of study (Labov, 1972; Eckert, 1989).

These two approaches have recently begun to converge in how they approach variability. In particular, psycholinguistic theories of speech perception have started to investigate the consequences of variability for comprehension. In part this realization comes from computational-level analyses of speech perception (Clayards et al., 2008; Feldman, Griffiths, \& Morgan, 2009; Feldman, 
Griffiths, Goldwater, \& Morgan, 2013; Norris \& McQueen, 2008; Kleinschmidt \& Jaeger, 2015). These approaches start from the hypothesis that the speech perception system is organized in order to be good at speech perception in the world that it has to operate in. In the spirit of ideal observer approaches to other domains (like visual perception, Marr, 1982; or memory, Anderson, 1990; Anderson, 1991) these approaches focus on spelling out how the nature of the task, the available information, and the structure of the world constrain, in principle, how well a listener can do.

Applied to speech perception, this approach offers two important insights. First, it suggests that speech perception can be thought of as a process of inference under uncertainty (Clayards et al., 2008; Norris \& McQueen, 2008): each linguistic unit is realized - even by a single talker-as a distribution of acoustic cues (cf. Lisker \& Abramson, 1964; Peterson \& Barney, 1952; Hillenbrand et al., 1995; Allen, J. L. Miller, \& DeSteno, 2003; Newman et al., 2001). This places a fundamental constraint on speech perception: the best a listener can do is to infer how likely each possible linguistic unit is as an explanation of the cues they observe, based on their knowledge of these cue distributions.

Second, this approach provides a new perspective on talker variability. One consequence of talker variability is that the distribution of cues for each linguistic unit changes from one situation to the next, depending on who's talking (Clopper, Pisoni, \& de Jong, 2005; Newman et al., 2001; Allen, J. L. Miller, \& DeSteno, 2003; Johnson, 2005; Foulkes \& Hay, 2015). Moreover, these differences cannot be entirely reduced to constant effects of physiological differences (like vocal tract size, Johnson, 2005; for review see Weatherholtz \& Jaeger, 2016). In this perspective, effective speech perception depends on good knowledge of the underlying cue distributions. Because these distributions change across situations, listeners must also constantly be inferring the current talker's linguistic generative model (the probabilistic distributions of cues they produce for each 
underlying linguistic structure). This is captured by the ideal adapter framework (Kleinschmidt \& Jaeger, 2015).

This second insight leads to the following prediction: an ideal adapter will take advantage of any additional structure in the world that is informative about how cue distributions vary from one situation to the next. This structure may be as simple as the fact that individual talkers tend to be consistent in the cue distributions they produce (Heald \& Nusbaum, 2015; see also Weatherholtz, Seifeldin, et al., 2016), meaning that prior experience with a familiar talker is informative about the cue distributions they will produce in the future. But structure can occur at other levels, too (e.g., male talkers produce vowels with systematically lower formant frequencies than female talkers; Peterson \& Barney, 1952; Hillenbrand et al., 1995). This is where psycholinguistic theories begin to parallel sociolinguistics. To the extent that variables like gender, class, regional origin, etc. are sociolinguistically relevant, they are reliably informative about how linguistic variables are realized, and hence helpful for speech perception.

This means that a listener can potentially learn a lot about a talker's cue distributions just by knowing who a talker is. Conversely, listeners can learn a lot about who a talker is based on the distributions of cues that they produce. This bi-directional relation between phonetic cues and social identity has long been recognized in sociolinguistics. This relationship also follows straightforwardly from the ideal adapter framework. However, the extent to which these two types of inferences-socio-indexically-conditioned linguistic inference and linguistically-conditioned inference about social identity-are feasible in practice depends on exactly how much a particular socio-indexical variable-a particular sense of who a talker is-actually influences the cue distributions that talkers produce.

One advantage of the ideal adapter framework over previous approaches 
to these issues is that it provides a set of computational and theoretical tools to quantify this relationship. The central question we address in this paper is twofold: 1) how much can listeners actually gain by considering socio-indexical variables for speech perception (both in absolute terms and relative to other grouping variables), and 2) how accurately could listeners infer socio-indexical grouping variables for a talker on the basis of their cue distributions. This work builds on the initial steps reported in Weatherholtz, Seifeldin, et al. (2016), developing the computational tools provided by the ideal adapter framework into a quantitative framework with the potential to unify explanations of linguistic and socio-indexical judgments.

\subsubsection{Our goals}

At a conceptual level, the goal of this paper is to show that the idea of inference provides a bridge between sociolinguistic and psycholinguistic perspectives on variation in speech. The ideal adapter framework treats both linguistic and socio-indexical judgments as inference, jointly informed by a listener's knowledge of cue distributions. In addition to this conceptual unification, the theoretical tools of this framework offer a common computational currency for developing models of linguistic and socio-indexical inference, and, critically, for grounding the parameters of those models in actual data. This common currency is the distribution of cues conditioned on linguistic and socio-indexical variables. These distributions can be measured, given the appropriate production data.

This brings us to the more concrete, immediate goals of this paper, which are to quantify the relationship between some prominent socio-indexical variables, linguistic variables, and acoustic-phonetic cue distributions. Most of the work on this relationship has been descriptive (but see, e.g., Clopper, Pisoni, \& de 
Jong, 2005; McMurray \& Jongman, 2011), aimed at establishing that differences between particular groups of talkers exist in the first place, and that listeners are sensitive to these differences at all. But the mere existence of differences does not establish exactly how informative or useful such grouping variables are for speech perception.

This paper is motivated by three interrelated questions about the relationship between socio-indexical variables and phonetic categories/cues. We begin by outlining each of these questions and their motivations, before presenting a series of computational studies that address them. In developing these studies, we lay out a novel, principled, and quantitative approach to understanding the relationship between socio-indexical variables and speech perception.

\section{Question 1: how informative are socio-indexical variables about cue distri- butions?}

According to the ideal adapter, effective adaptation to different talkers-and hence robust speech perception-depends on knowing how and how much cue distributions vary across talkers for each phonetic category/contrast. If a category's cue distribution is strongly conditioned on a socio-indexical variable like talker identity (i.e., varies a lot between talkers), then listeners need to be prepared to rapidly adapt. If, on the other hand, a category's cue distributions are not strongly conditioned on talker, then listeners are better off using their prior experience with other talkers and not rapidly adapting their beliefs about that particular category. The same logic applies for other socio-indexical variables. If a category's cue distributions are strongly conditioned on, for instance, the sex of the talker, then a listener's previous experience with other male talkers will be highly informative about an unfamiliar male talker's realization of that category, while their experience with female talkers would be misinformative. 
In principle, it is possible that the amount and structure of between-talker variability is more or less the same across phonetic categories and cues. In such a world, listeners should adapt uniformly to talker variability in different cues/contrasts, drawing - or not- on previous experience with other talkers in the same way across phonetic categories. This would also mean that every phonetic category provides an equally good substrate for communicating socio-indexical meaning. If, on the other hand, phonetic categories/contrasts vary in how strongly their cue distributions vary across talkers, then listeners should adjust their adaptation to an unfamiliar talker's phonetic cue distributions differently across categories/contrasts.

There is qualitative evidence suggesting that the structure of talker variation is, in fact, different depending on the phonetic contrast and cues. For example, vowels exhibit substantial variability conditioned on the gender and the regional background of the talker (Peterson \& Barney, 1952; Hillenbrand et al., 1995; Clopper, Pisoni, \& de Jong, 2005; Labov et al., 2005, among others). Talker variation in word-initial stop voicing, on the other hand, is largely unstructured (at least in American English), exhibiting little systematic variation by gender, regional dialect, or age (Allen, J. L. Miller, \& DeSteno, 2003; Chodroff et al., 2015). There is moreover qualitative evidence that the overall level of cross-talker variation is also different between these two contrasts. For instance, visual inspection of the results of Chodroff et al. (2015) (Figure 1) suggests that cross-talker variance in voiceless word-initial stop VOT is roughly half of within-category variation (standard deviations of approximately $10 \mathrm{~ms}$ and 20ms VOT respectively), whereas inspection of similar results for vowels from Hillenbrand et al. (1995) (Figure 4) suggests that cross-talker variability in vowel production is approximately double the within-category variability.

However, no previous work has quantified the relative level of betweentalker vs. within-talker variability across phonetic contrasts. In part, the lack 
of quantitative comparisons reflects the fundamental difficulty of comparing very different phonetic contrasts and cues in a principled way. We address this here by quantifying how informative socio-indexical variables-including talker identity-are about the distribution of acoustic-phonetic cues. We do this for two sets of contrasts/cues: vowels (cued by first and second formant frequencies; F1xF2) and word-initial stop voicing (cued by voice onset time; VOT). We selected these particular contrasts based on the qualitative evidence that they exhibit different degrees and types of cross-talker variability. Our approach is based on that of Weatherholtz, Seifeldin, et al. (2016), and compares conditional cue distributions (e.g., for male vs. female talkers) to marginal cue distributions (e.g., for all talkers) using an information theoretic measure of similarity between distributions. Focusing on information theoretic properties of the cue distributions provides a principled common currency for comparing different socio-indexical variables, phonetic categories, and cues.

\section{Question 2: How useful are socio-indexical variables for speech recognition?}

The utility of a socio-indexical variable for guiding speech recognition is not necessarily the same as its informativity about the underlying cue distributions themselves. By the utility of a socio-indexical variable, we mean the benefit for speech perception from 1) tracking the cue distributions for phonetic categories conditional on group (e.g., for male and female talkers) and 2) knowing the value of that variable for a talker (e.g., whether a talker is male or female). Informativity provides an upper bound on utility, since tracking conditional cue distributions can only improve recognition to the extent that they are actually different from marginal (un-conditioned) distributions.

It is possible, in principle, that two equally informative socio-indexical variables may not be equally useful for the purposes of speech perception, and, for instance, not equally tracked by listeners. For instance, if one category varies 
across talkers in a way that never causes it to overlap with another category, listeners can safely ignore talker-level variation in that category without increasing the chance of errors in speech recognition. On the other hand, when a category varies across talkers or groups in a way that does cause it overlap with other categories, ignoring talker-conditioned distributions would lead to slowed or inaccurate speech recognition. The same is true of broader socioindexical groupings, like sex or dialect.

We know that listeners do, at least in some cases, use socio-indexical variables to guide speech perception. For example, perception of vowels (and fricatives) is affected by the perceived gender of the talker, which can be cued by voice quality, visual presentation of a male or female face, or even explicit instruction (Strand, 1999; Johnson et al., 1999; Strand \& Johnson, 1996). Listeners use other socio-indexical variables to guide recognition of vowels as well: Niedzielski (1999) found that if listeners believe that a talker is Canadian, they hear more Canadian raising than if they believe the talker is American. Hay and Drager (2010) found a similar sensitivity to dialect group using an even subtler manipulation, manipulating listeners perceptions based on a stuffed animal that cued New Zealand or Australia. More broadly, listeners are better at comprehending speech in noise from familiar talkers than unfamiliar talkers (Nygaard \& Pisoni, 1998).

What we do not know is whether the extent to which listeners are sensitive to socio-indexical variables for different contrasts is predictable based on the underlying structure of talker variability for these contrasts. This depends on exactly how useful these socio-indexical variables would be for speech recognition, which is the second question we address in this paper. The ideal adapter provides a principled way to link talker- and group-conditioned variability in cue distributions with the utility of those variables. This link comes from treating speech perception as an inference process, which predicts phonetic classi- 
fication from the underlying cue distributions. We use this link to quantify, in Study 2, how much speech recognition is improved by conditioning cue distributions on a particular socio-indexical variable. As in Study 1, our approach is based on Weatherholtz, Seifeldin, et al. (2016), extended here to a wider range of cues, categories, and socio-indexical variables.

One advantage of the ideal adapter framework is that quantifying the utility of socio-indexical variables in this way leads to further, testable predictions about the role that socio-indexical variables play in speech perception. One sense of utility that is of particular interest is how listeners generalize from experience with one talker to another in speech recognition. A variety of experiments on phonetic recalibration (or perceptual learning) have shown that listeners sometimes generalize from experience with, for instance, a male talker to inform perception of an unfamiliar, female talker (Kraljic \& Samuel, 2006; Kraljic \& Samuel, 2007; Reinisch \& Holt, 2014; C. M. Munson, 2011), but sometimes they do not (Eisner \& McQueen, 2005; Kraljic \& Samuel, 2007; Reinisch \& Holt, 2014). The ideal adapter predicts that listeners will generalize when they think that the two talkers both belong to a group that is informative about the particular contrast being recalibrated. Assessing the utility of different levels of grouping (including gender) is a necessary first step towards making these predictions precise and testable

\section{Question 3: How well could listeners infer socio-indexical variables given cue distributions?}

The utility of tracking socio-indexically-conditioned cue distributions goes beyond the recognition of phonetic categories. The purpose of spoken language is not limited to communicating linguistic information. Every time we speak we also communicate socio-indexical information, information about aspects of our social identity such as gender, age, class, etc. (cf. Eckert, 1989; Eckert, 
2012a; Labov, 1972). The approach outlined so far also lends itself to reasoning about these sorts of social inferences as well. In particular, a listener can use knowledge about group-conditioned cue distributions in order to evaluate which group best explains the cues they have observed from a talker, and hence which group they are likely to belong to. ${ }^{1}$

As with the previous two questions, there is some evidence that listeners can in fact make these sorts of socio-indexical inferences on the basis of speech alone (for a review, see Thomas, 2002). For instance, listeners can classify talker's regional dialect at above-chance accuracy based on a short except of their speech (a single sentence read from a standard set Clopper \& Pisoni, 2006; Clopper \& Pisoni, 2007). We include a talker's identity as a socio-indexical variable (in the sense of what individual person they are). There is evidence that listeners can infer a talker's identity from sine-wave speech (Remez, Fellowes, \& Rubin, 1997), speech which has been processed to remove most non-phonetic voice quality cues to identity but preserve most phonetic information (Remez, P. E. Rubin, Pisoni, \& Carrell, 1981).

However, the extent to which knowledge of group-conditioned phonetic cue distributions would actually be useful in practice depends on the actual distribution of cues within and between groups, as does the relative usefulness of different aspects of the speech signal. Thus our final study aims to quantify how well listeners would be able to infer socio-indexical properties based on phonetic cue distributions alone. We do this by treating these socio-indexical inferences in mathematically the same way that we treat linguistic inferences. This highlights the potential of the ideal adapter framework as a unifying perspective on linguistic and socio-indexical inferences.

\footnotetext{
${ }^{1}$ There are arguably other, more dynamic non-linguistic aspects that are coded in the speech signal, such as mood or attitude towards our interlocutors (Eckert, 2010; Podesva, 2007). The approach laid out here-at least in principle — extends to these aspects of communication as well.
} 
We chose to focus on socio-indexical variables that are categorical and generally stable over time for a single talker. This choice is based on feasibility: our analysis depends on having sufficient data to estimate cue distributions for multiple talkers, within groups defined by socio-indexical variables. Sociolinguistics increasingly recognizes that the meanings of socio-indexical variables are dynamically constructed and not necessarily static within a single individual (either producer or perceiver; Eckert, 2012a; Podesva et al., 2001; Podesva, 2007; Foulkes \& Hay, 2015; Levon, 2014). The techniques we introduce here can - in principle-be applied to more dynamic and contextually conditioned variables, but they present unique and interesting challenges that are beyond the scope of the current paper.

\subsection{General methods}

We next describe the datasets we use and the motivations for selecting these datasets. Then, we introduce our general modeling approach. The specific methods by which we quantify informativity, utility, and inferability of socioindexical grouping variables are discussed in their respective studies.

\subsubsection{Data}

Guided by our goals, we selected two datasets. The first is a collection of vowel formants from the Nationwide Speech Project (NSP; Clopper, Pisoni, \& de Jong, 2005), and the second a collection of voice onset times (VOT) for word-initial voiced and voiceless stops from the Buckeye corpus (Pitt et al., 2007, extracted by Wedel, in prep). We chose to compare vowels and stop voicing because there appears to be different amounts and types of talker variability for these contrasts. Our specific goals further constrain our particular choice of datasets. 
First, in order to estimate the between vs. within talker variability, we need data with enough talkers who each produce enough tokens of the relevant phonetic categories in order to estimate the corresponding cue distributions. Second, in order to estimate the utility of socio-indexical variables, we need data where each talker produces tokens from each of the categories for the contrast. Third, to assess the influence of different socio-indexical variables, we need data where different socio-indexical variables are actually annotated. These rule out many existing datasets which either do not contain enough tokens per talker, or (especially for stop voicing) only contain tokens from a subset of categories (e.g., only voiceless stops).

Based on the variables annotated in the available data, we consider cue distributions conditioned on the following socio-indexical grouping variables:

- Marginal: is a control grouping, which includes all tokens from all talkers. This serves as a baseline against which more specific group distributions can be compared, and as a lower bound for speech recognition accuracy.

- Sex: this is coded as male/female for both vowels and stop voicing, allowing us to compare the role of sex for two different contrasts.

- Age: this is only relevant for VOT, because the talkers in the NSP are uniformly young. In the Buckeye corpus, age is coded as a binary variable (younger/older than 40, VOT only).

- Dialect: the NSP contains data from talkers from six dialect regions (see below for details).

- Dialect+Sex: Clopper, Pisoni, and de Jong (2005) found that sex modulates dialect differences, so we also examining cue distributions conditioned on dialect and sex together (12 levels, vowels only).

- Talker: we also consider cue distributions conditioned on talker identity, as an upper bound on informativity and utility. 


\section{Vowels}

For vowels, we used data from the Nationwide Speech Project (NSP; Clopper, Pisoni, \& de Jong, 2005). Specifically, we analyzed first and second formant frequencies (F1xF2, measured in Hertz) recorded at vowel midpoints in isolated, read "hVd" words. This corpus contains 48 talkers, 4 male and female from each of 6 regional varieties of English: North, New England, Midland, MidAtlantic, South, and West (see map in Clopper, Pisoni, \& de Jong, 2005; regions based on Labov et al., 2005). Each talker provided approximately 5 repetitions of each of 11 English monophthong vowels (plus ey), for a total of 2659 observations.

One of our primary goals is to assess the informativity of different grouping variables. Sex differences in vocal tract size are a major source of variability in vowel production, and thus may be more informative than other factors. However, this likely depends on the details of how acoustic cues are represented. Differences in vocal tract size, for instance, lead to overall shifts in the resonant frequencies and hence formant frequencies across all vowels, but leave the relative positions of vowel categories more or less intact (e.g., Hillenbrand et al., 1995). Moreover, there is evidence that domain-general auditory normalization or adaptation processes removes some or all of this overall shift, and hence using un-normalized formant frequencies may overestimate the informativity of sex relative to other grouping factors.

For this reason, we also used Lobanov-normalized formant frequencies as input, in addition to the un-normalized formant frequencies in Hertz. Lobanov normalization z-scores F1 and F2 separately for each talker (Lobanov, 1971), which effectively aligns each talker's vowel space at its center of gravity, and scales it so they have the same size (as measured by standard deviation). This controls for overall offset in formant frequencies caused by varying vocal tract 
sizes (from both sex differences and individual variation). It does this while preserving the structure of each talker's vowel space, so that (for instance) dialect-specific vowel shifts are maintained.

\section{Stop voicing}

We analyzed data on word-initial stop consonant voicing in conversational speech from the Buckeye corpus (Pitt et al., 2007, extracted by Wedel, in prep). Voice onset time (VOT) was automatically extracted for 5984 word initial stops, 2264 voiced and 3720 voiceless, for labial, coronal, and dorsal places of articulation. Data came from 24 talkers, who were balanced male and female and younger/older than 40 years. On average, each talker produced 42 tokens for each phoneme (range of $5-156$ ).

Our primary reason for considering VOT/voicing at all is to get a sense of the range of informativity and utility of socio-indexical variables across different phonetic categories. VOT is thought to be relatively stable across talkers, and formant frequencies relatively variable. The strength of this particular VOT corpus is that it contains observations of both voiced and voiceless stops from the same talkers. This allows us to directly assess how much talker variability in VOT distributions (Allen, J. L. Miller, \& DeSteno, 2003) actually impacts recognition of voiced vs. voiceless stops, and hence estimate an upper bound on the usefulness of any socio-indexical grouping variable for this contrast.

However, the downside is that this corpus does not not contain data from talkers who vary on socio-indexical variables that are known to correlate with differences in VOT distributions, like native language background. Preliminary analyses of a collection of VOTs for only voiceless stops from FrenchEnglish bilinguals (Lev-Ari \& Peperkamp, 2013) suggests that, even though these groups are known to produce different VOT distributions, the size of this effect is much smaller than even talker-level variability within the monolingual 
talkers in the Buckeye corpus (which, to foreshadow our results, is substantially smaller than for vowels).

\subsubsection{Modeling approach}

All of our analyses depend on knowing the distribution of cues for each phonetic category, at different levels of socio-indexical grouping. We obtain estimates of these distributions in the following way. We assume that each phonetic category can be modeled as a normal distribution over cue values (stop voicing as univariate distributions over VOT, and vowels as bivariate distributions of F1 and F2). These distributions are parameterized by their mean and their covariance matrix (or, equivalently, variance in the case of VOT). We fit these parameters to data from our corpora via maximum likelihood, using the sample mean and covariance of tokens from each category. We do this separately for each group. For example, for gender, we obtain one estimate of the ae distribution based on all the tokens from male talkers, and one from all tokens from female talkers. Likewise, for dialect, we estimate one distribution based on all talkers from the North dialect region, another one from tokens from Mid-Atlantic talkers, and so on.

Assuming that each category is a normal distribution is not a critical part of our approach, but rather a standard and convenient assumption. In particular, the normal distribution has a small number of parameters and this allows us to efficiently estimate the distribution for each category with a limited amount of data (e.g., five tokens per talker-level vowel distribution). 


\subsection{Study 1: Informativity of socio-indexical group- ings about cue distributions}

Our first goal is to assess how informative socio-indexical variables are about the cue distributions for each phonetic category, across levels of socio-indexical grouping and phonetic categories/cues.

\subsubsection{Methods}

One way to quantify how informative a socio-indexical grouping variable is about cue distributions is by comparing the group-level cue distributions with the marginal distribution of cues from all groups. The reason for this is that if a socio-indexical grouping variable (e.g., sex) is not informative about cue distributions, then the the cue distributions for each group (e.g., male and female talkers) will be essentially identical, and hence indistinguishable from the overall cue distribution. If, on the other hand, a socio-indexical variable is informative about cue distributions, then the distribution for each group will deviate substantially from other groups, and by extension from the overall distribution as well. The particular measure we use to compare distributions is the Kullback-Leibler (KL) divergence, which explain next. ${ }^{2}$

\section{Technical details}

$\mathrm{KL}$ divergence (or relative entropy) is an information-theoretic comparison of two distributions (MacKay, 2003, p. 34). In our usage, it measures the expected

\footnotetext{
${ }^{2}$ This measure is intuitively similar to the proportion of variance explained by the socioindexical grouping variable (as used in McMurray \& Jongman, 2011). However, it is a more general approach that does not require that we assume that the underlying distributions are normal distributions, and can be applied even to categorical variables (like distributions of words or syntactic structures). It also, as we show here, naturally extends to multidimensional cue spaces, taking into account the correlations between cues, and supporting comparisons to other cue spaces.
} 
cost (in bits of extra message length) of encoding data from each group using a code that's optimized for the marginal distribution (MacKay, 2003, p. 98). ${ }^{3}$

In general, the KL divergence of $Q$ from $P$ is

$$
D L(Q \| P)=\int p(x) \log \frac{p(x)}{q(x)} \mathrm{d} x
$$

(with density functions $q$ and $p$ respectively). Intuitively, we can think of the KL divergence in the following way. Take data that has been sampled from a true distribution $P$. The $\mathrm{KL}$ divergence of another distribution $Q$ from $P$ is how much better, on average, $P$ explains the sampled data than $Q$. The KL divergence increases as $Q$ diverges more from $P$, and has a minimum value of zero, which is only achieved when $P=Q$ (MacKay, 2003, p. 34).

In our case, $P=\mathcal{N}_{G}$ is a multivariate $e^{4}$ normal cue distribution conditioned on a socio-indexical group, with mean $\mu_{G}$ and covariance $\Sigma_{G}$, while $Q=\mathcal{N}_{M}$ is the marginal (not conditioned on group) cue distribution with mean $\mu_{M}$ and covariance $\Sigma_{M}$. With some simplification, ${ }^{5}$ the KL divergence of the marginal from the group distribution works out to be

$$
D L\left(\mathcal{N}_{M}|| \mathcal{N}_{G}\right)=\frac{1}{2}\left(\operatorname{tr}\left(\Sigma_{M}^{-1} \Sigma_{G}\right)+\left(\mu_{M}-\mu_{G}\right) \Sigma_{M}^{-1}\left(\mu_{M}-\mu_{G}\right)-d+\log \frac{\left|\Sigma_{M}\right|}{\left|\Sigma_{G}\right|}\right)
$$

where $d$ is the dimensionality of the distribution, and log is the natural logarithm. For ease of interpretation, we report KL divergence in bits, which corresponds to using $\log _{2}$ in equation eq. 5.1, or dividing equation eq. 5.2 by $\log (2)$ ).

\footnotetext{
${ }^{3}$ Richter, Feldman, Salgado, and Jansen (2016) apply a similar approach to vowel data from the NSP, with an important difference: they compare group-specific distributions to each other, instead of the marginal distribution. Their goal is to evaluate how well different normalization schemes remove talker variability, while ours is to evaluate how informative different socioindexical variables are about the underlying distributions. Our goals are thus related but not identical

${ }^{4}$ The math is the same for the univariate special case, as with VOT.

${ }^{5}$ See, for instance, http://stanford.edu/ jduchi/projects/general_notes.pdf, p. 13.
} 
For each phonetic category, we calculate the KL divergence of each group's cue distribution from the marginal distribution of cues from all talkers. We then average these single-category scores for each group to calculate the overall divergence for that group. ${ }^{6}$ Finally, for each grouping level, we average the divergence across groups, and compute bootstrapped confidence intervals over groups for this mean.

\subsubsection{Results}

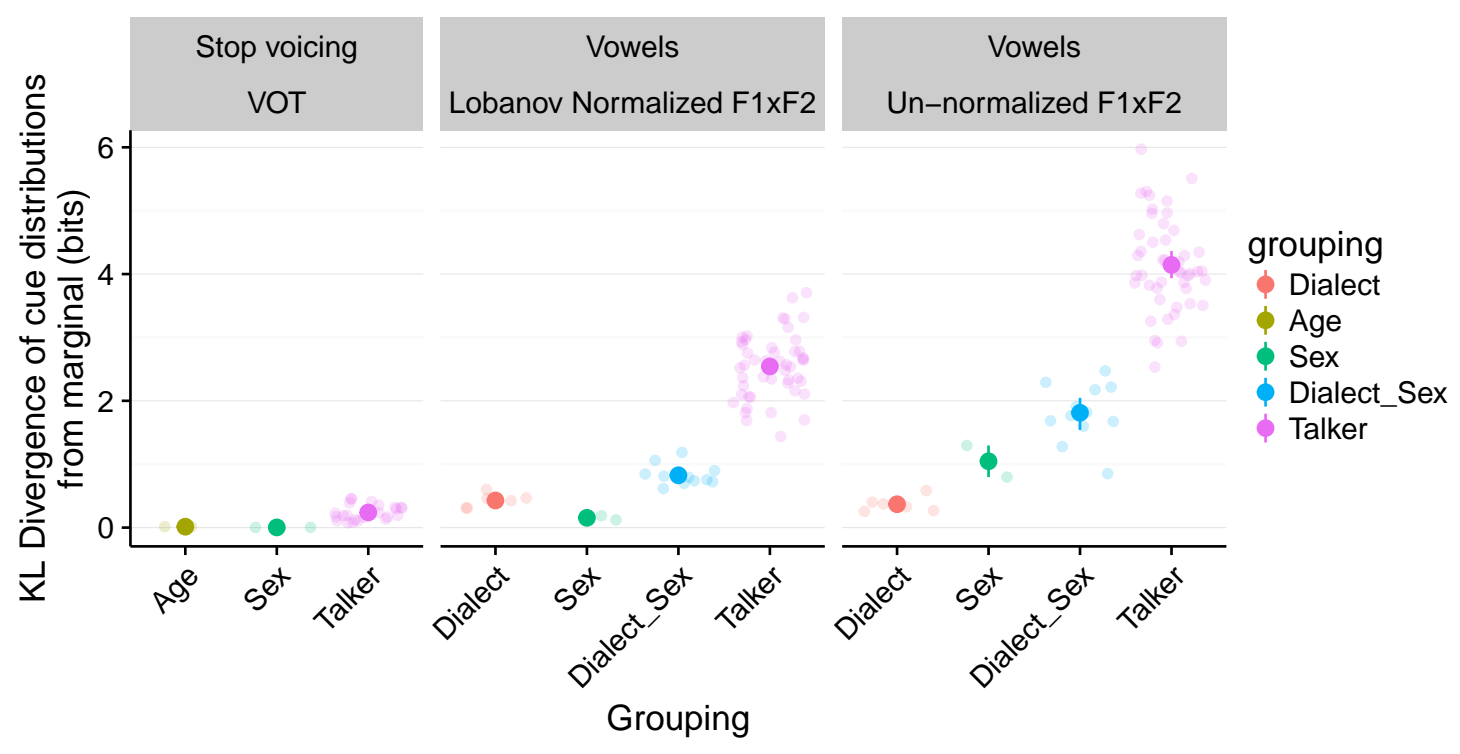

Figure 5.1: Socio-indexical variables are more informative about cue distributions for vowel (formants) than for stop voicing (vot). On top of this, more specific groupings (like Talker and Dialect+Sex) are more informative than broader groupings (Sex). This is indicated by higher KL divergence of each grouping level from marginal (each point shows one group's average KL divergence from marginal distributions, and large points with errorbars show the mean and bootstrapped $95 \%$ CIs over groups).

\footnotetext{
${ }^{6}$ We average over categories for two reasons. First, it's mathematically convenient, because the KL between to normal distributions can be computed in closed form, but for mixture models must be estimated with numerical integration. Second, by averaging over categories, we control for any effects that could be due to the overall difference between the number of vowel (11) and stop voicing (2) categories.
} 
Figure 5.1 plots the KL divergence of cue distributions at different levels of grouping from marginal distributions, across contrasts (vowels and stop voicing) and cues (VOT, raw/Lobanov-normalized F1xF2). There are two clear patterns.

First, we find that talker identity is an order of magnitude more informative about vowel distributions than about VOT distributions. That is, knowing a talker's identity is significantly provides significantly more information about their vowel formant frequency distributions than it does about their VOT distributions. This is important because it uses a quantitative measure to confirm the qualitative finding from previous work that there is less talker variability in VOT than in formant frequencies (e.g., Allen, J. L. Miller, \& DeSteno, 2003; Lisker \& Abramson, 1964; vs. Peterson \& Barney, 1952; Hillenbrand et al., 1995). Strikingly, the most informative variable for VOTtalker identity-is roughly as informative as the least informative variable for Lobanov-normalized F1xF2 (Sex). Figure 5.2 illustrates what this means in terms of the conditional distributions for these two combinations of grouping variables and categories: on average, individual talkers' VOT distributions diverge from the marginal distribution (B) at roughly the same level that the male and female distributions of normalized F1xF2 diverge from the marginal normalized F1xF2 distributions (A).

Second, on the whole, we find that more specific groupings are more informative than less specific groupings. This suggests that, within a group, talkers are more consistent than they are between groups. This is to be expected when there is talker variability, and when that variability is greater between groups than within groups. The exception to this pattern is that, for un-normalized formants, sex is more informative than dialect, even though dialect is more specific ( 8 talkers per dialect, vs. 24 per sex). The likely reason for this is that sex differences in physiology (e.g., vocal tract length) change formant frequencies for all 
A

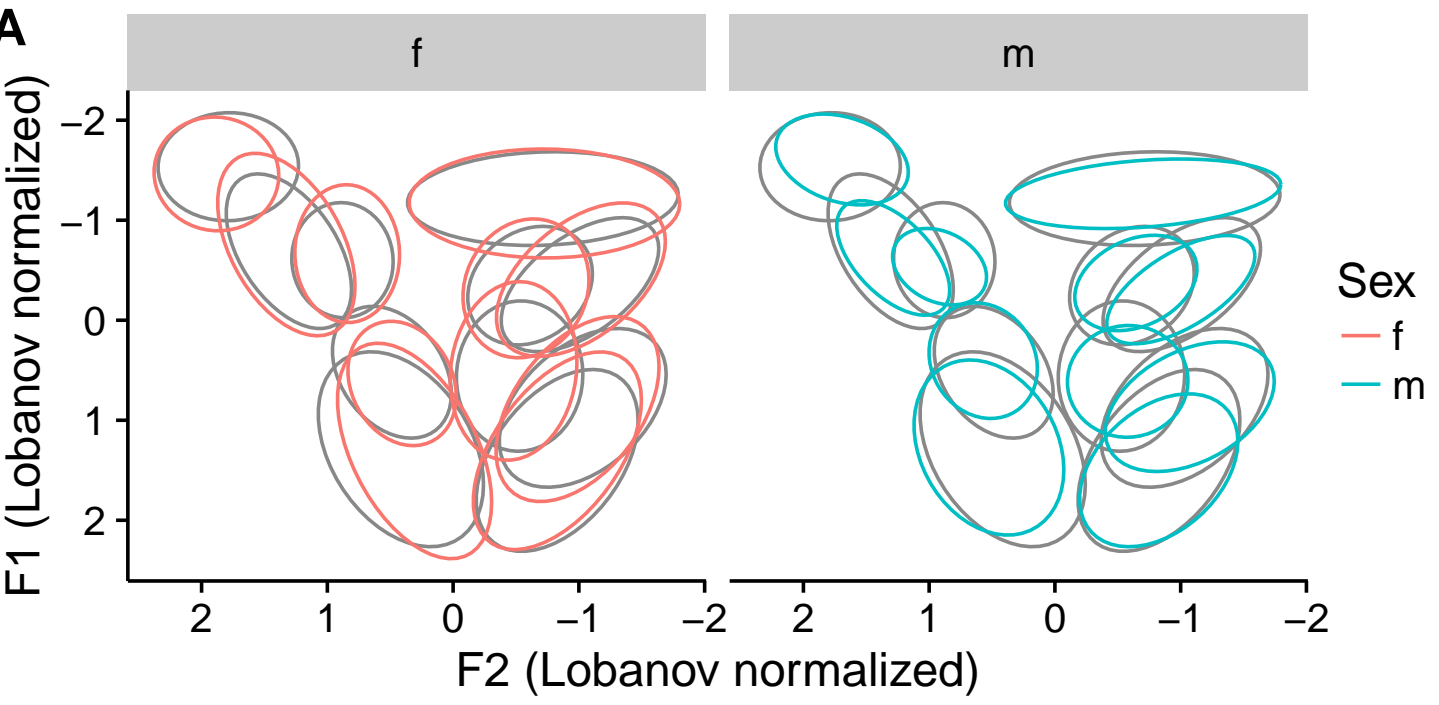

B

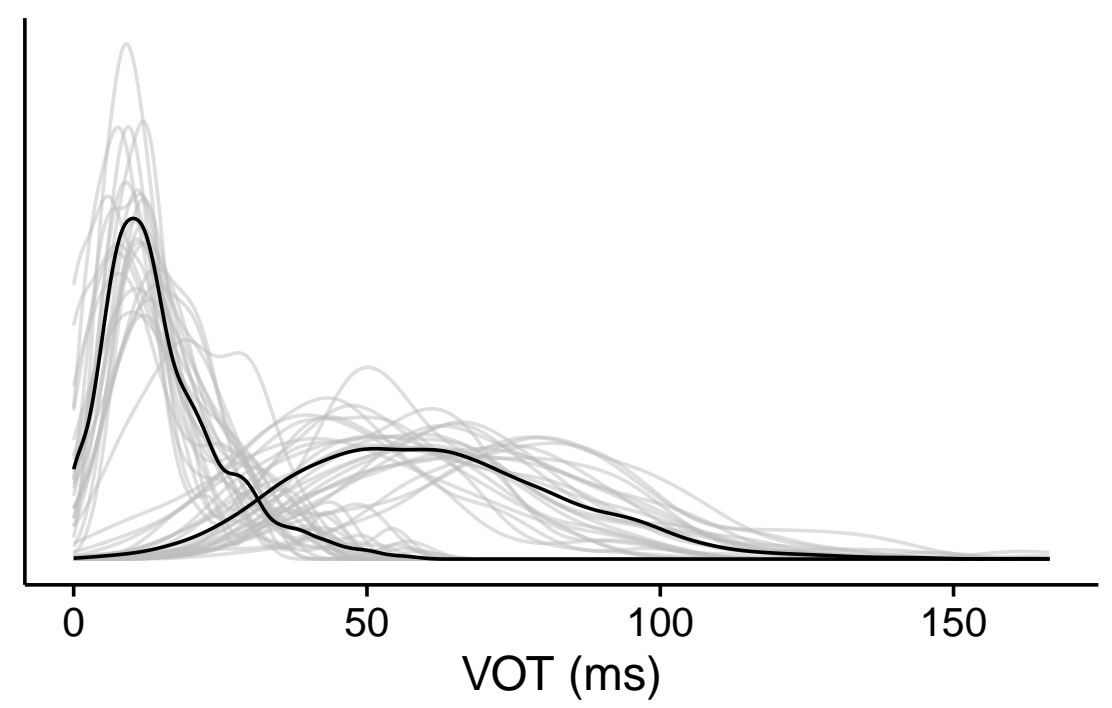

- Marginal

- Talker

Figure 5.2: Male and female distributions of Normalized F1xF2 diverge from the marginal distributions (A) only slightly less than talker-specific VOT distributions diverge from marginal (B) (see Figure 5.1). 
vowels (Johnson, 2006), but dialect variation is limited to certain dialect-vowel combinations (Clopper, Pisoni, \& de Jong, 2005; Labov et al., 2005).

As Figure 5.3 shows, while the relative ordering of grouping variables' informativity is consistent across vowels, their actual degree of informativity varies quite a bit. Dialect (and Dialect+Sex) is particularly informative for aa, ae, eh, and uw, vowels with distinctive variants in at least one of the dialect regions from the NSP. As noted by Labov et al. (2005), aa is undergoing a merger with ao in some regions, ae and eh participate in the Northern Cities Chain Shift, and uw is fronted in some regions (and in others only by female talkers; Clopper, Pisoni, \& de Jong, 2005).

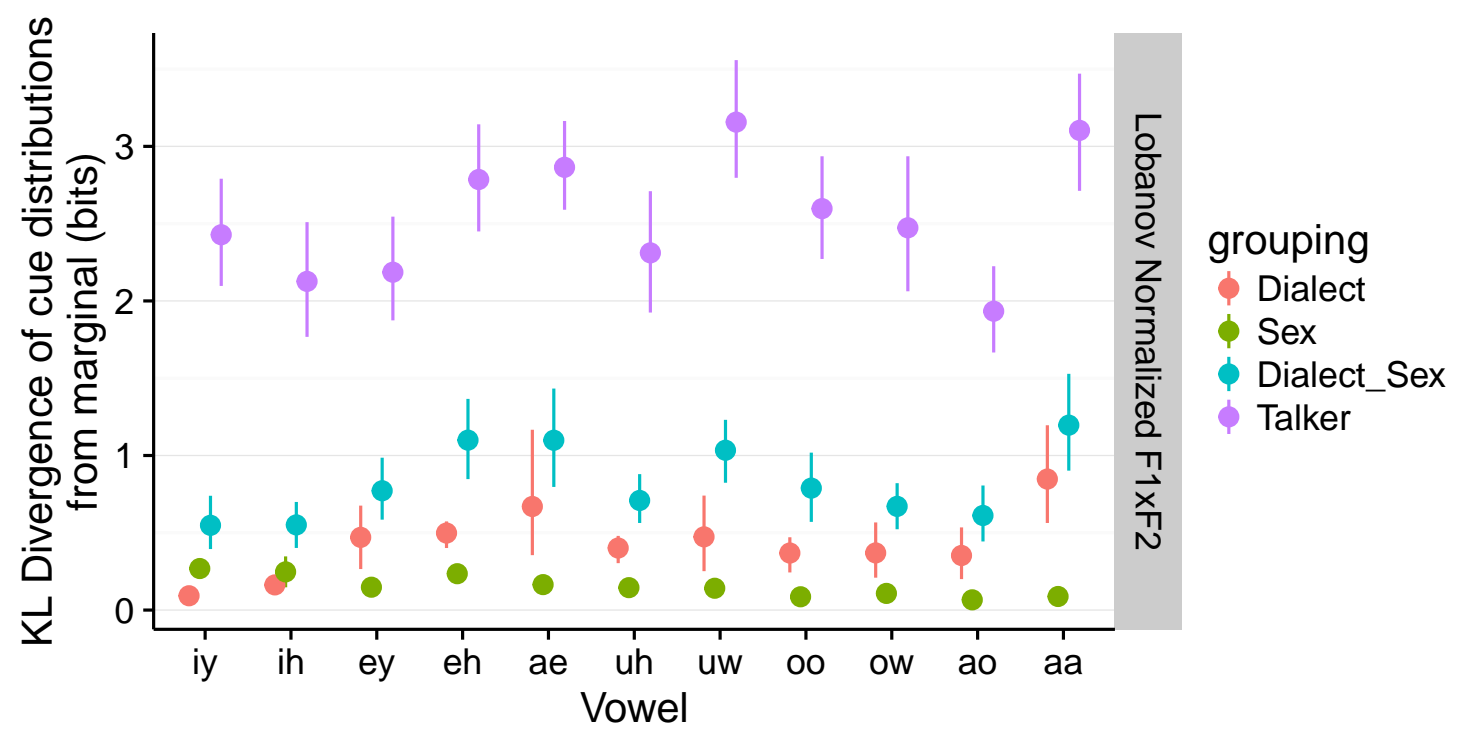

Figure 5.3: Individual vowels vary substantially in the informativity of grouping variables about their cue distributions. Only normalized F1xF2 is shown to emphasize dialect effects.

Figure 5.4 shows the KL divergence of each dialect's vowel distributions, demonstrating that dialects do indeed vary in how informative they are, both overall and by vowel. Some of this variability corresponds to known patterns of dialect variability. In particular, talkers from the North dialect region produce vowels-ae and aa in particular-with formant distributions that deviate 
markedly more from the marginal distributions than any of the other dialects. Both of these vowels participate in the Northern Cities Shift (Labov et al., 2005; Clopper, Pisoni, \& de Jong, 2005). The Mid-Atlantic aa is, like the Northern aa, non-merged with ao (Clopper, Pisoni, \& de Jong, 2005) and hence deviates from the marginal aa substantially.

Other particularly high divergences do not correspond to known sociolinguistic variants, and may be flukes of this particular dataset which includes a small number of talkers per region. This includes the high divergence of uw from New England talkers, which is the result of these talkers producing a very low-variance uw distribution. Similarly, Mid-Atlantic talkers produce a lowvariance ey distribution that is slightly higher and fronter than the marginal distribution.

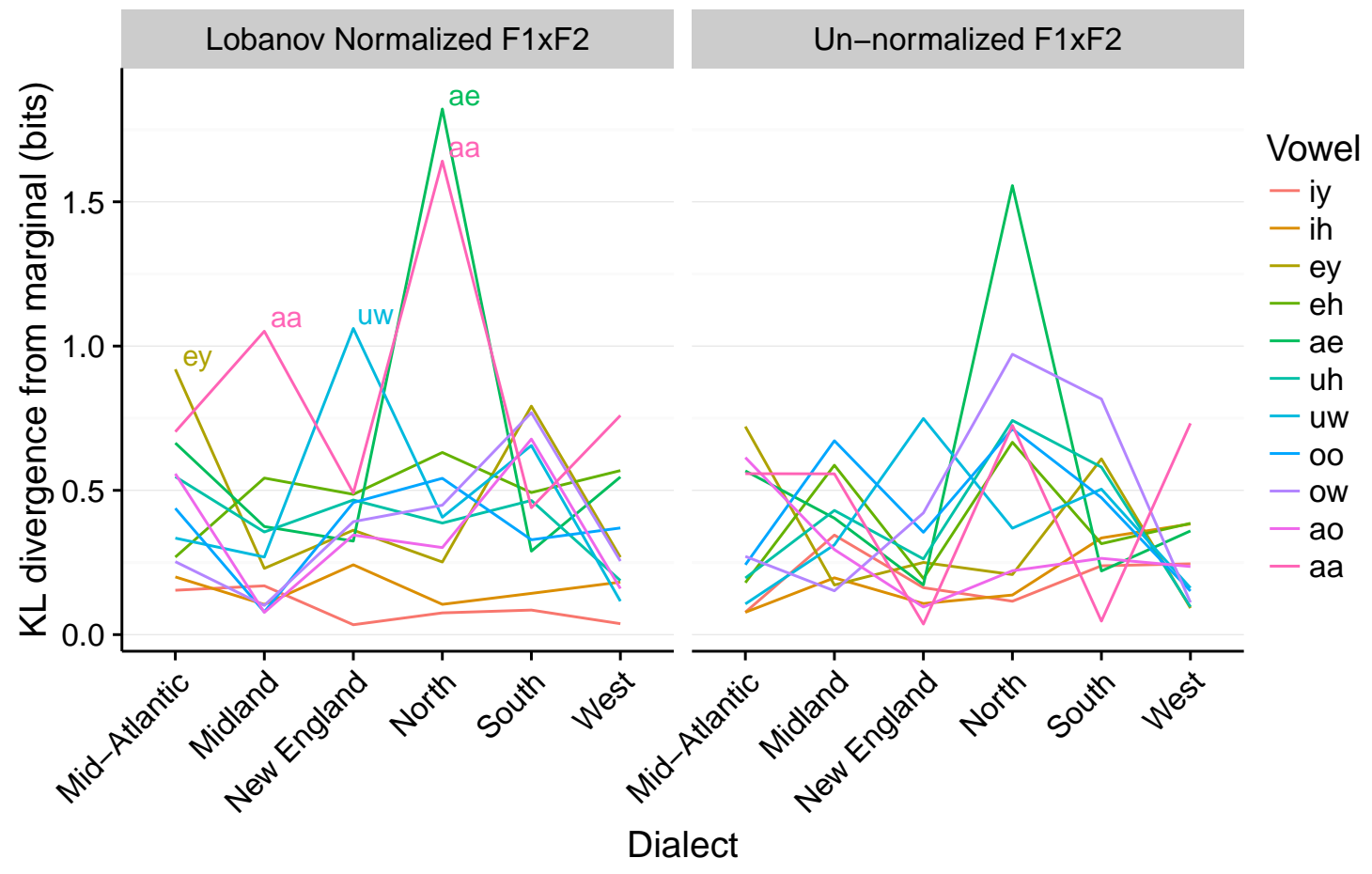

Figure 5.4: A small number of dialect/vowel combinations account for most of the divergence of dialect-specific vowel formant distributions. In particular, the distribution of ae and aa produced by Northern talkers diverge markedly more than any other vowel/dialect combination. 
Finally, these results show that, even after controlling for overall shifts in formant frequencies across all vowels via Lobanov normalization, there is still substantial talker variability. Normalization also substantially reduces the informativity of sex alone, which is to be expected given that most of the difference between male and female vowel distributions is due to the overall shifts in formant frequencies that Lobanov normalization removes. Interestingly, while normalization reduces the informativity of dialect and sex considered together, their combination is still more informative than dialect alone. This suggests that dialect differences themselves are modulated by sex (as noted by Clopper, Pisoni, \& de Jong, 2005).

This asymmetry in informativity across both dialects and vowels raises the question of how listeners adapt to variation across categories and cue dimensions. All else being equal, a listener should be more confident in their prior beliefs about a category that varies less across talkers, and hence adapt less flexibly (Kleinschmidt \& Jaeger, 2015). But it is not clear at what level listeners track variability for the purpose of determining how quickly to adapt. For instance, as we have seen, overall vowels vary substantially more across talkers than stop categories, but there are differences in how much individual vowels vary. It remains to be seen whether listeners adapt to all vowels with the same degree of flexibility, or are sensitive to these vowel-specific differences in cross-talker variability.

\subsection{Study 2: utility of socio-indexical groupings for speech recognition}

Next, we evaluate the utility of each grouping variable for speech recognition. One way to estimate the utility of a socio-indexical variable is based on actual 
human speech perception behavior. The downside of this method is that it is impossible to control listeners' prior experience, and hence what group-level distributions they may already be tracking. It can moreover difficult to manipulate listeners' beliefs about the socio-indexical properties of the talker whose speech they are classifying, especially for variables like dialect or class.

We take a different approach that avoids these problems (at the cost of certain well-understood assumptions, described below), based on patterns of socio-indexically condition variation in cue distributions themselves. One principled way quantify utility is in terms of probability of correct recognition of phonetic categories. By treating speech perception as an inference problem, the ideal adapter (Kleinschmidt \& Jaeger, 2015; and the ideal listener models that it draws on, e.g. Clayards et al., 2008) provides a link between group-specific cue distributions and the probability of correct recognition. We describe this link and how we actually calculate it, and then present the results for the datasets we consider here.

\subsubsection{Methods}

In the ideal adapter/listener, the link between cue distributions and recognition of phonetic categories is provided by Bayes Rule. ${ }^{7}$ Bayes Rule says that the posterior probability that a particular cue value came from a category is the likelihood of that cue value being generated by that category's cue distribution,

\footnotetext{
${ }^{7}$ Our approach is based on a generative model of categorization, which starts from cue distributions and derives classification boundaries. This contrasts with discriminative models which directly map cue values to categories, like the logistic regression model that McMurray and Jongman (2011) used to evaluate the utility of different cue encoding schemes for classifying fricatives. The main advantage to using a generative framework, overall, is that it provides a learning model, and does not require us to assume that listeners actually know the underlying distributions for each talker (Kleinschmidt \& Jaeger, 2015). But it remains a question for future work exactly how the predictions of our approach here differ from those of the discriminative modeling approach of McMurray and Jongman (2011).
} 
divided by the total likelihood of it being generated by any category. ${ }^{8}$ For instance, the posterior probability that a VOT of $20 \mathrm{~ms}$ is a /b/ is the likelihood of a $20 \mathrm{~ms}$ VOT under the /b/VOT distribution, divided by the total likelihood of $20 \mathrm{~ms}$ VOT under both /b/ and /p/.

If two categories' distributions overlap a lot, then they will frequently assign similar likelihood to cue values generated by the other, leading to confusion and recognition errors. Separating distributions based on a socio-indexical grouping variable can affect the overlap between different phonetic categories, and thus the rate of correct recognition is a measure of the utility of that grouping. If lumping together tokens from, for instance, both male and female talkers causes vowel distributions to overlap more, that will decrease the probability of correct recognition, relative to the male- and female-only distributions (that is, distributions conditioned on gender).

More broadly, by calculating the probability of correct recognition using, for instance, the cue distributions of each category produced by female talkers, we can estimate how well a listener would be able to recognize speech from an unfamiliar female talker if all they knew was the talker's sex. This measure is meaningfully different from the KL-based informativity measure from Study 1 , because a category might vary substantially between, e.g., male and female talkers, but if it is already highly distinguishable from other categories this variability may not impact correct recognition.

As we discuss in more detail in the general discussion, this measure is best considered a lower bound on how useful a particular grouping variable is for comprehending speech from an unfamiliar talker.

\footnotetext{
${ }^{8}$ This assumes-for the sake of convenience- that the prior probability of each category is equal. This is not a limitation of our approach or the ideal adapter/observer framework. Bayes rule naturally extends to situations where there is a prior expectation that some categories are more likely than others. It would be possible to incorporate, for instance, the base frequencies of the phonetic categories we examine into account, based on phonotactic corpora (e.g., Vitevitch \& Luce, 2004).
} 


\section{Technical details}

We want to determine the phonetic category $v_{i}{ }^{9}$ of each of the cues $x_{i}$ produced by a talker. If we assume that the listener knows that this talker belongs to group $g=j$, this inference is a straightforward application of Bayes Rule:

$$
p\left(v_{i} \mid x_{i}, g=j\right) \propto p\left(x_{i} \mid v_{i}, g=j\right) p\left(v_{i}\right)
$$

If, on the other hand, the listener does not know which group the talker belongs to, they have to marginalize out group. This amounts to taking a weighted average of the posterior probabilities under each group, weighted by the probability that the talker belongs to that group, $p(g \mid x)$ :

$$
p\left(v_{i} \mid x_{i}\right)=\sum_{j} p\left(v_{i} \mid x_{i}, g=j\right) p(g=j \mid x)
$$

(where $x$ refers to all the tokens produced by this talker). For the sake of brevity, we do not present results when group is unknown, limiting ourselves to the case where the listener knows the value of the relevant socio-indexical variable. This is not a completely unreasonable assumption, because listeners often have some information about the sex, regional origin, etc. of a talker, based on nonlinguistic cues.

For vowels, we classified vowel categories directly. For voicing, the only cue available is VOT, which does not (reliably) distinguish place of articulation. Thus, we classified voicing separately for each place of articulation, and then average the resulting accuracy.

To assess statistical significance, we bootstrap over talkers. This is a non-

\footnotetext{
${ }^{9} x_{i}$ refers to a single observed cue value (possibly multidimensional, in the case of vowel formants), and $x$ (without subscript) refers to a vector of multiple observations (from a single talker, unless otherwise specified). $v_{i}$ refers to observation $i^{\prime}$ s category, and $g$ to a talker's group. $\sum_{j}$ refer to a sum over all possible values of $j$.
} 
parametric approach that provides confidence intervals and $p$ values for effects at the population level, based on the limited sample of talkers in our datasets, without assuming a particular parametric form (like a normal distributions) for the variability across talkers.

\section{Avoiding anti-conservative estimates through cross-validation}

For classification, if test data is included in the training set, this artificially inflates accuracy at test (James, Witten, Tibshirani, \& Hastie, 2013, Section 5.1). Cross-validation controls for this by splitting data into training and test sets. For group-level models (sex, age, dialect, and dialect+sex), we use leave-one-talker-out cross-validation, training each group's models with test talker's observations held out. For the talker-specific models, we use 6-fold cross-validation (or leave-one-out when there were fewer than 6 tokens in a category for a talker), where each phonetic category is randomly split into 6 approximately equal subsets. Then, one subset of each category is selected for test, the models are trained on the remaining five, and the test data is classified as above.

Cross-validation is not only important because it provides an unbiased measure of classifier accuracy. It is also essential for testing the hypothesis that group-level cue distributions are useful to listeners. If the test talker is included in the training dataset, then the utility of that talker's own productions is confounded with any utility of the group itself.

\section{Avoiding biases from different group sizes}

For the vowel data, the different levels of grouping have very different group sizes, and this requires some caution. The broadest (sex) has 24 talkers per group (23 after holdout), while the most specific (dialect+sex) has only 4 ( 3 after 
holdout). This introduces a systematic bias in favor of broader groupings, because small sample sizes lead to noisier estimates of the underlying model, and hence lower accuracy (on average) at test (James et al., 2013, Section 2.2.2). This bias would make it impossible to meaningfully compare probability of correct recognition across grouping levels, defeating the purpose of this analysis.

To correct for this, we randomly subsampled three talkers (without replacement) within each group in the training set (that is, after holding out the test talker). This ensures that every group has is the same size as the smallest group across grouping levels. For the studies reported here, that corresponds to the test talker's Dialect+Sex group. ${ }^{10}$

We use 20 different random subsamples for each test talker, averaging accuracy over each resampled training set. A different subsampling is used for every talker, and thus any additional variance introduced by this procedure is accounted for by bootstrapping talkers. The estimates obtained in this way allow us to compare accuracy across groupings with different group sizes, but at the cost of underestimating the true group-level accuracy across the board. As such, they must be considered a useful lower bound on the utility of socioindexical groupings.

Subsampling was not necessary for the VOT data: the Buckeye Corpus is balanced by age and sex, the two socio-indexical variables we consider.

\subsubsection{Results}

Figure 5.5 shows the probability of correct recognition for stop voicing/vowel, based on the cue distributions at each level of grouping. As with informativity

\footnotetext{
${ }^{10} \mathrm{We}$ also ran the analyses resampling each group to 7 talkers, which corresponds to the Dialect-level group size after holdout (excluding the Dialect+Sex grouping from comparison, since there are only 4 talkers per group before holdout). Besides a small increase in overall accuracy (because of the reduced variance of the distribution estimates), this did not substantially change the results.
} 


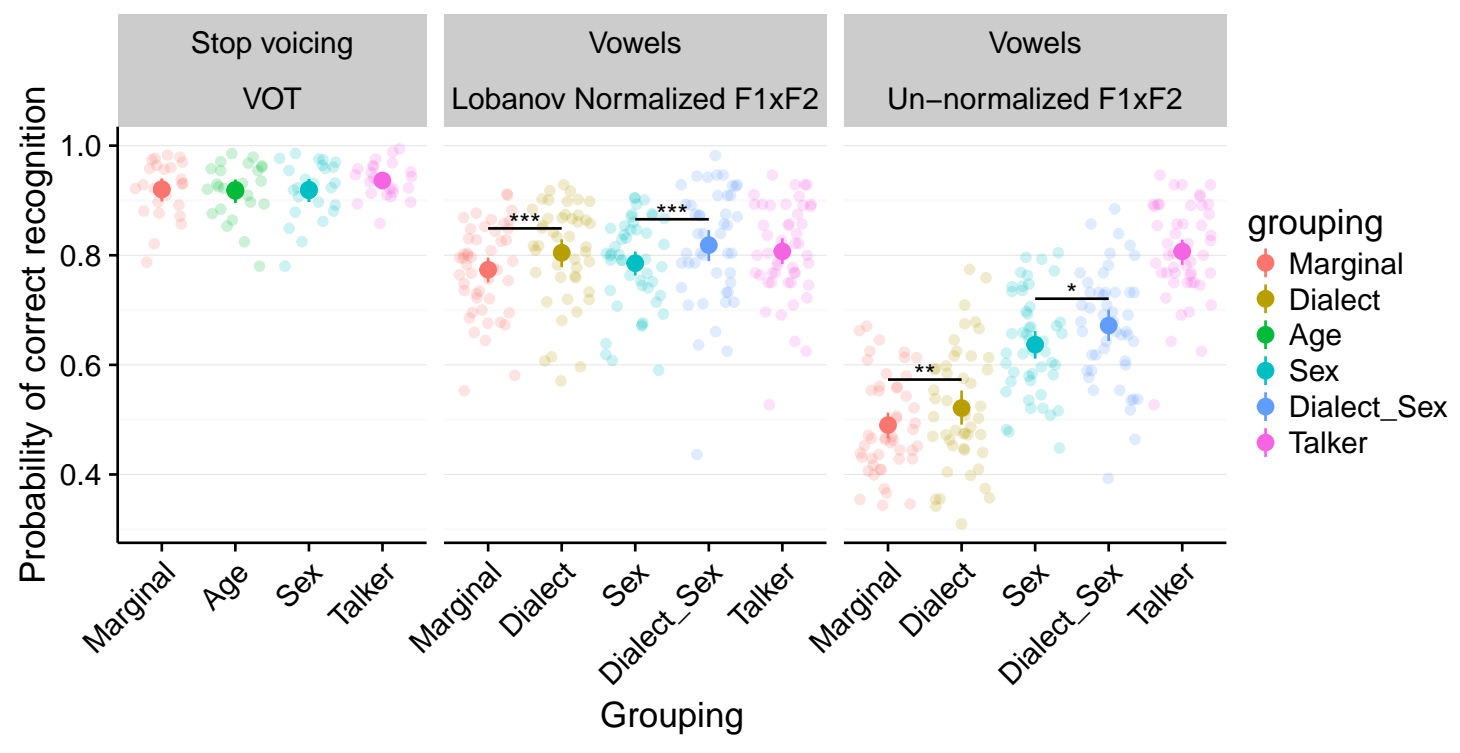

Figure 5.5: Speech recognition accuracy using for marginal, group-level, and talker-specific cue distributions. Small points show individual talkers, and large points and lines show mean and bootstrapped 95\% CIs over talkers. Marginal and group-level accuracy is based on leave-one-talker out crossvalidation, and talker-specific on 6-fold cross-validation (or leave-one-tokenper-category out if there are fewer than 6 tokens per category). Bars and stars show significant increases in accuracy when conditioning on dialect, alone or in addition to sex. For clarity, only some significant comparisons are shown. Here and elsewhere: $* p<0.05, * * p<0.01$, and $* * * p<0.001$. 
about the distributions themselves, there's an asymmetry between vowels and stop voicing in the overall utility of socio-indexical variables for speech recognition. Probability of correct recognition is overall higher for stop voicing than vowels. Voicing recognition is also less sensitive to the particular grouping variable: knowing whether a talker is male vs. female (or young vs. old) provides no advantage when classifying their VOTs as voiced or voiceless. This is consistent with the finding above that VOT distributions themselves do not differ across groups. Even using a talker's own distributions provides only a minimal advantage (on the order of $2 \%$ increase in accuracy over marginal, age-, and sex-conditioned distributions, all three $\mathrm{p}<0.01) .{ }^{11}$

For vowels, normalized input results in higher recognition accuracy across the board, again paralleling the findings about the cue distributions themselves. The one exception is at the level of talker-specific distributions, where recognition accuracy is unchanged (since Lobanov normalization is a linear transformation of the input, which leaves the structure of the categories within each talker unchanged).

Conditioning vowel cue distribution on sex-either alone, relative to marginal, or in addition to dialect-provides the biggest boost in recognition for un-normalized formant accuracy. For normalized input, none of the socioindexical grouping factors provide much of an advantage over the marginal distributions. In both cases, dialect provides a small but consistent advantage for recognition, both alone and in combination with sex (increasing accuracy by $3 \%$ on average, all $p<0.05)$.

The utility of socio-indexical grouping for recognizing individual vowels largely mirrors the overall pattern (Figure 5.6). As with informativity, the utility of dialect varies substantially from one vowel to the next. For most vowels,

\footnotetext{
${ }^{11}$ These comparisons are also significant when using log-odds of correct recognition, rather than raw probabilities.
} 


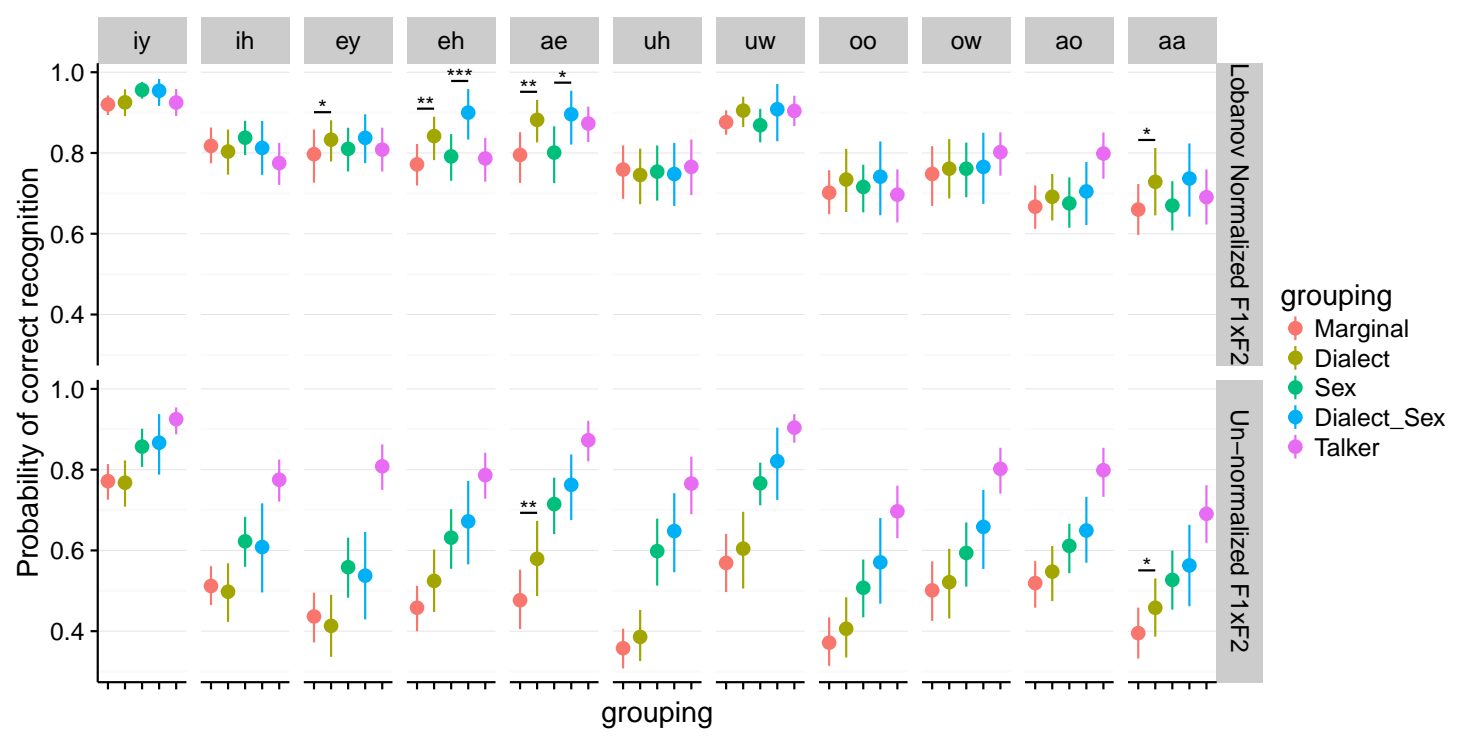

Figure 5.6: Probability of correct recognition varies across vowels, overall and according to the socio-indexical grouping variable. Bars and stars show significant improvement from conditioning on dialect, above marginal or in addition to sex alone.

conditioning on dialect makes little difference to correct recognition. But for a few-particularly ae and eh-conditioning on dialect increases accuracy by nearly $10 \%$. In the case of normalized formant input, accuracy using dialectconditioned distributions actually equals or surpasses the accuracy with talkerspecific distributions.

The overall utility of dialect also varies substantially based on the talker's actual dialect (Figure 5.7). This is consistent with the fact that dialects themselves vary in how much they deviate from both the norms of Standard American English (Clopper, Pisoni, \& de Jong, 2005) and from the marginal cue distributions in this dataset (Figure 5.4). Most notably, conditioning on dialect provides a consistent advantage for talkers from the North dialect region, on the order of $10 \%$.

As with the cue distributions themselves in Study 1, this suggests that there may be a differential advantage to tracking cue distributions for different vow- 
els, and hence listeners might be more sensitive to variation in some categories than others. On the other hand, it is possible that listeners' degree of flexibility depends on the cue (or phonetic feature, like \pm voicing) rather than the category specifically, in which case they would be predicted to track all cues sharing the same cue with the same level of flexibility.

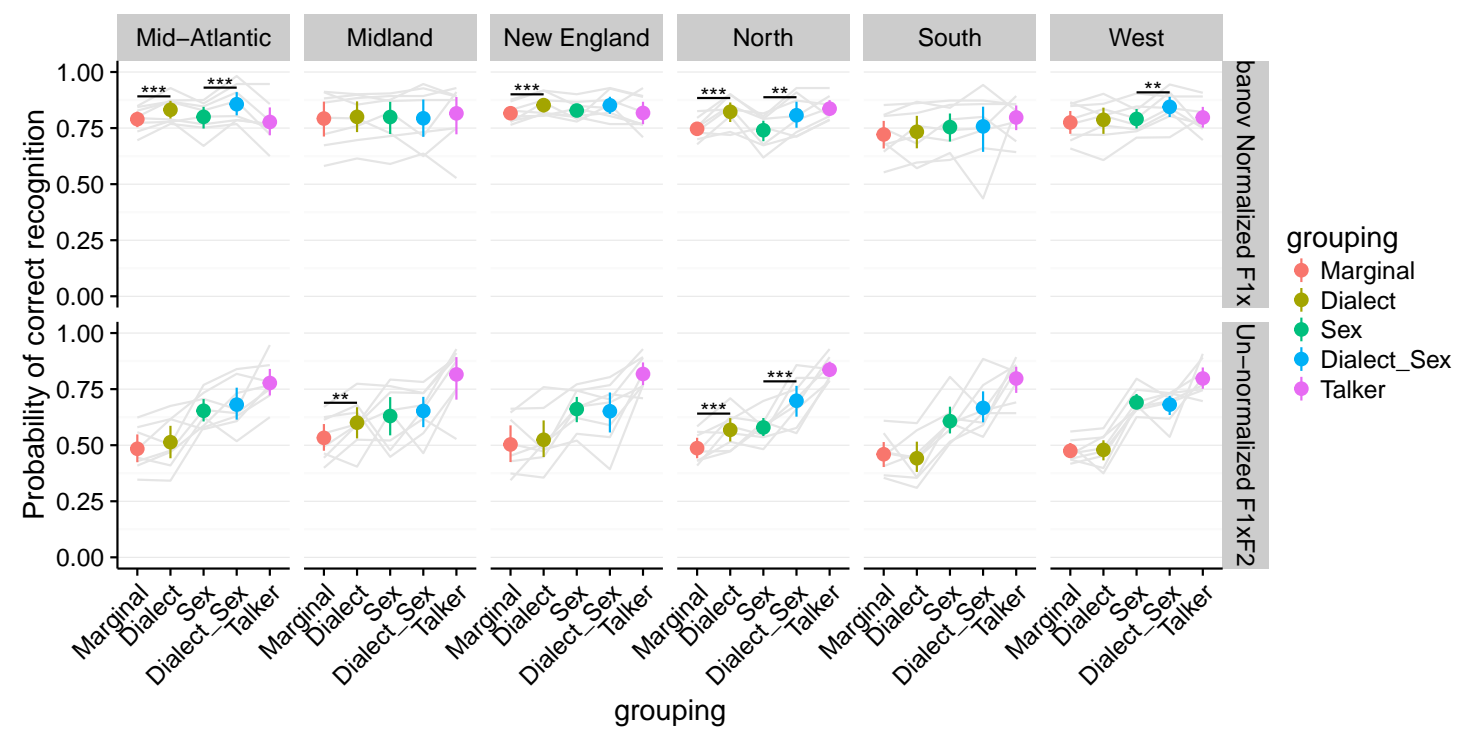

Figure 5.7: The utility of socio-indexical variables varies across dialects. Dialect itself is particularly informative only for talkers from the Mid-Atlantic and North regions. Each line shows a single talker, to emphasize within-talker changes in accuracy with grouping level, and large points and confidence intervals show mean accuracy and bootstrapped 95\% CIs over talkers.

\subsection{Study 3: Inferring socio-indexical variables from cue distributions}

Finally, we assess how well listeners would be able to determine values of socio-indexical variables based on cue distributions alone. Qualitatively, a listener should be able to determine a talker's socio-indexical group based on phonetic cues to the extent that the distributions of those cues differ across 
groups. Here we quantify this using the same computational tools that we used to quantify the utility of socio-indexical grouping for speech perception. Specifically, we ask how well a listener could infer a talker's group, based on the distributions of cues produced by other talkers in the same and different groups.

\subsubsection{Methods}

The process of inferring socio-indexical group parallels the process of inferring phonetic categories: the posterior probability that a talker belongs to a particular group is proportional to the likelihood of the cues they produce under that group's cue distributions, relative to the total likelihood under all groups' distributions.

We formalize this with the same kind of ideal observer model used for phonetic recognition. That is, we compute the posterior probability of each socioindexical group $g=j$, given all of the talker's observed cue values $x$ :

$$
p(g \mid x) \propto p(x \mid g) p(g)=\left(\prod_{i} p\left(x_{i} \mid g\right)\right) p(g)
$$

The only complication is that, without knowing the the phonetic category of each observation a priori, each observation may have been generated by any of the phonetic categories. Thus, to determine the overall likelihood of observing a cue value $x_{i}$ under group $g$, we first have to marginalize over categories $v_{i}$ :

$$
p\left(x_{i} \mid g\right)=\sum_{k} p\left(x_{i} \mid v_{i}=k, g\right) p\left(v_{i}=k \mid g\right)
$$

We perform this analysis separately for each level of socio-indexical grouping. For instance, for each talker we compute both $p(\operatorname{sex} \mid x)$ and $p($ dialect $\mid x)$. 
We assess whether accuracy is significantly above chance using a binomial test, rather than bootstrapping as in the previous studies, because accuracy is measured at the subject (rather than token) level and thus follows a (nonhierarchical) binomial distribution. We also computed the bootstrapped $p$ values and confidence intervals, and they did not differ substantially from the exact ones. Note that chance level accuracy depends on the number of groups, and this varies by socio-indexical variable.

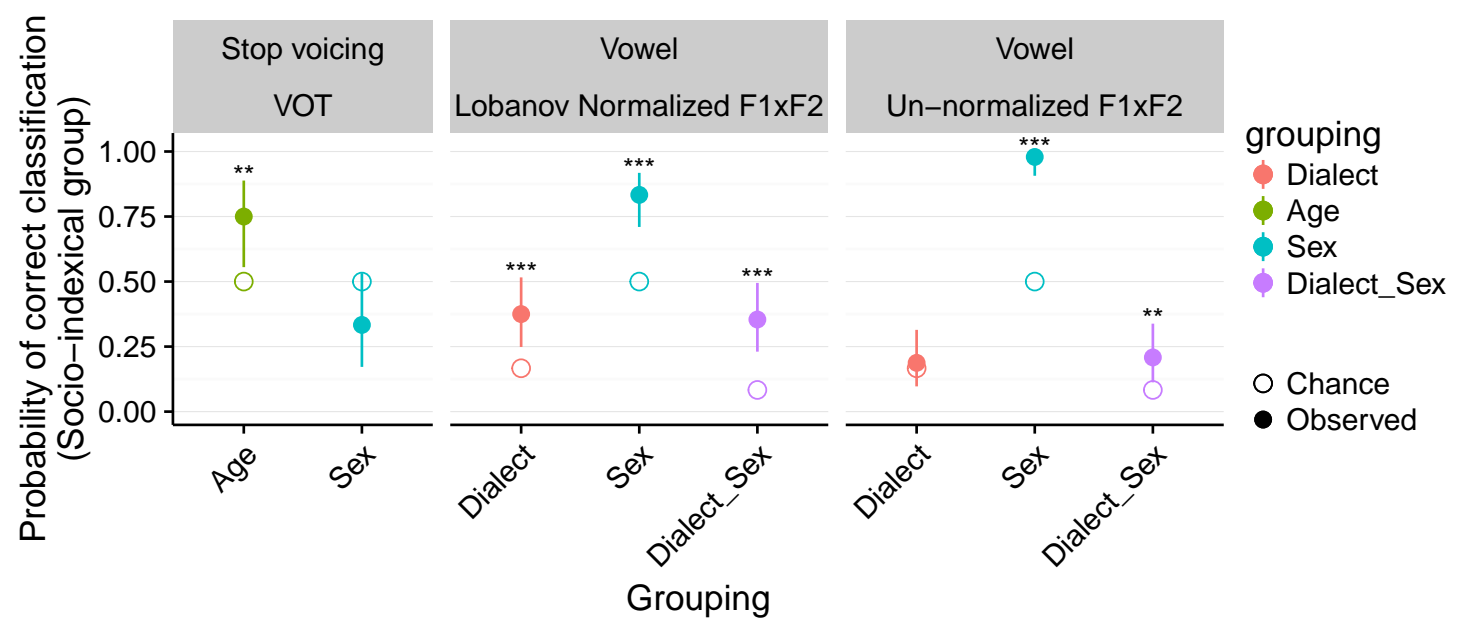

Figure 5.8: Probability of correctly classifying a talker's socio-indexical group varies with the grouping variable, contrast, and cues. A talker is correctly classified if the overall posterior probability of their actual group given their unlabeled productions is the highest of all groups.

\subsubsection{Results}

For many groupings, it is possible to infer each talker's group with above chance accuracy, given only that talker's unlabeled observed cues (Figure 5.8; all $p<0.01$, except for inferring a talker's sex based on their VOT distributions, and dialect from un-normalized F1xF2 distributions, both $p>0.15$ ). Surprisingly, despite the fact that VOT distributions do not differ markedly by age (cf. Figure 5.1), it is still possible to infer a talker's age based on VOT with 
above chance accuracy $(75 \%, 95 \%$ CI $58-92 \%$, p < 0.01).

In some respects, these results mirror the patterns of informativity about the cue distributions themselves (Figure 5.1). Vowel formant distributions vary more according to group than do stop VOT distributions, and likewise socioindexical group can, on the whole, be inferred with higher accuracy based on vowel formants than on VOT. For vowels specifically, much of the variability across talkers is driven by sex differences, and this is the grouping variable that's easiest to infer of the three we tested.

However, in other respects, these results do not simply mirror informativity. For instance, un-normalized F1xF2 distributions diverge from marginal more for dialect+sex than they do for sex alone, but accuracy at inferring a talker's sex is nearly at ceiling, while accuracy is barely above chance for inferring a talker's dialect+sex. Likewise, normalized F1xF2 distributions given dialect and dialect+sex diverge from marginal less than non-normalized, but accuracy at inferring these two grouping variables is higher for normalized than nonnormalized.

Why the discrepancy? The informativity measure we used was the average divergence of each category's cue distribution. For inferring indexical groups, we assumed that listeners do not know the intended category of each observation, and so the relevant likelihoods are each based on a mixture of the categoryspecific distributions. Even if there are some categories whose individual distributions diverge across groups, the overall mixture distribution of all categories may still be too similar to allow for the group to be reliably inferred.

\subsection{General Discussion}

Our results show that, on the whole, socio-indexical grouping variables are informative about phonetic cue distributions, useful for improving speech recogni- 
tion, and inferable from phonetic cues themselves. The extent to which these are true depends on the particular grouping variable and particular phonetic categories/cues involved. At least for our American English data, socio-indexical variables are more useful, more informative, and more easily inferred for vowels than for stop consonant voicing. Some variables are broadly useful (sex, talker identity) while others are useful only in certain, specific contexts (dialect for certain vowels/dialect combinations).

Our results also speak to the relationship between informativity, utility, and inferability themselves. In general, informativity and utility mirror each other: conditioning on a socio-indexical variable is more useful for speech recognition when the corresponding conditional cue distributions diverge more from the overall or marginal distributions. But being useful for speech recognition does not always mean that a socio-indexical variable can be easily inferred from phonetic cues alone, or vice-versa.

In the remainder of this paper, we discuss the implications of these results. First, the ideal adapter generally predicts that listeners should track conditional distributions for groups that are informative and useful for speech recognition. By directly quantifying the utility and informativity of a number of grouping variables, our results are an important step towards making more specific predictions about what group-level representations listeners should maintain if, as assumed by the ideal adapter, they are taking advantage of the structure that is actually present in cross-talker variability. Second, our results shed light on discrepancies between phonetic contrasts in listeners' willingness to generalize recalibration/perceptual learning from one talker to another. Third, this paper provides a proof of concept for the idea that, like phonetic judgments, sociolinguistic judgments can be productively viewed as a sort of inference under uncertainty. This suggests the potential for a tighter integration of sociolinguistic and psycholinguistic perspectives on speech perception. 


\subsubsection{What to track?}

Treating speech perception as a problem of inference under uncertainty-as the ideal adapter does-highlights the importance of listeners' knowledge about the distributions of cues that are produced for each linguistic unit. A major question that this perspective raises is what linguistic, socio-indexical, and acoustic/phonetic variables listeners are learning distributions for. The ideal adapter does not directly answer this question, but provides a set of conceptual and quantitative tools for addressing it. The studies reported here take these tools and apply them to data on how many different talkers produce two different sets of phonetic categories. We hope that by doing so we provide a proof of concept for the broad usefulness of these tools. One purpose they might be put to is to formulate hypotheses about what distributions listeners should track.

Even prior to taking into account cognitive limitations, the ideal adapter predicts that listeners should not track everything. Rather, listeners need only track the joint distributions of variables that are informative. At the level of phonetic categories themselves, this means that (for instance) there's no reason for listeners to track each vowel's distribution of preceding $\mathrm{VOT}^{12}$ (or more absurdly, completely unrelated physical quantities like temperature or barometric pressure). This also applies at the level of socio-indexical grouping variables: listeners get no benefit for tracking separate distributions for different groups of talkers for a cue that does not systematically vary between those groups.

In fact, it can actually hurt a listener to track cue distributions at a level that's not informative. The reason for this is related to the idea of bias-variance tradeoff from machine learning (James et al., 2013, Section 2.2.2). Given the same amount of data, tracking multiple, specific distributions will result in noisier,

\footnotetext{
${ }^{12}$ Barring, of course, the possibility that VOT is systematically affected by neighboring vowels, and hence informative about them.
} 
less accurate estimates than lumping together all the observations in a single distribution. This price may be worth paying for a listener when there are large enough differences between groups that treating all observations as coming from the same distribution biases the estimates of the underlying distribution (and hence the inferences that listeners make based on those distributions) far enough away from the true structure of the data. To take a concrete example, modeling each vowel as a single distribution of (un-normalized) formants across all talkers results in high-variance, overlapping distributions which have low recognition accuracy. But modeling them as two distributions-one for males, and one for females-provides more specific estimates and higher classification accuracy, as shown by Figures 5.1 and 5.5.

Thus, the ideal adapter predicts that listeners should learn separate cue distributions for levels of a socio-indexical grouping variable when that variable has high informativity about some categories' cue distributions and / or high utility for speech recognition. However, the notions of informativity and utility apply beyond better speech recognition per se. Listeners extract a lot of nonphonetic/linguistic information from speech signal. To properly define the informativity or utility of a particular grouping variable, we need to consider the goals of speech perception, which go beyond just recognizing phonetic categories (or linguistic representations at any particular level, cf. Kuperberg \& Jaeger, 2016). Sociolinguistics recognizes that, in many cases, the communication of social information is just as-if not more-important than the communication of linguistic information. Groupings that are socially meaningful can thus be informative and justify being tracked, even if ignoring them has a negligible effect on speech recognition, as long as the corresponding cue distributions carry some information about relevant social variables. In our results, dialect is a good example: on the whole, ignoring dialect doesn't have huge consequences on recognition accuracy. But it can be inferred based on vowel F1 and 
F2, and listeners are plausibly interested in determining a talker's regional origins for a variety of reasons.

An additional consideration is that listeners are not simply told which variables are informative and which are not. They must actually infer what distributions are actually worth tracking. Moreover, every listener's experience with talker variability will be different, and so a variable that is informative in one listener's experience may be irrelevant in another's. While the analyses we present here go a long way toward focusing the predictions of the ideal adapter framework, they must be combined with knowledge of each listener's own personal history—either assumed, or somehow measured, even approximatelyin order to make specific predictions for a particular subject or population of subjects. This same logic applies to which socio-indexical variables are of direct interest to a listener: social categories that are highly important in one person's social world may be completely meaningless in another's. An important aspect of the research program laid out by the ideal adapter framework is to probe listeners' prior beliefs directly (which the previous chapter is a first step towards).

It is important to note that listeners' associations between linguistic and socio-indexical variables do not always seem to be based on objective informativity of those variables. Rather, some variants can become disproportionately salient or enregistered, can further vary based on context (Eckert, 2012a; Podesva et al., 2001; Podesva, 2007; Foulkes \& Hay, 2015; Levon, 2014; Jaeger \& Weatherholtz, 2016). These deviations between objective informativity and subjective salience remain to be explained and specified in more detail. Our work here provides a set of tools for assessing objective informativity, a critical first step in understanding this relationship. More generally, by treating listeners' decisions about which socio-indexical and linguistic variables to jointly track as a form of inference (like linguistic and socio-indexical judgments themselves), 
this perspective provides a framework for testing different ways that listeners' experience and beliefs shape their subjective notions of socio-indexical associations with linguistic variables.

Finally, our results suggest that the input representation-the cue space over which categories are distributions-can affect which variables are informative or not. For vowels, using Lobanov-normalized formants as input substantially reduces the informativity and utility of sex as a grouping factor, but increases the utility of dialect in many cases. From a listener's perspective, dialect would appear to be relatively uninformative without normalization. This points to a complex interaction between normalization and adaptation/perceptual learning as strategies for coping with talker variability. Both strategies are, in fact, used by listeners, but the interaction between them is poorly understood (Weatherholtz \& Jaeger, 2016).

\subsubsection{Consequences for adapting to unfamiliar talkers}

The results of this study also tell us something about how listeners might adapt to an unfamiliar talker. The ideal adapter links informativity to adaptation, and the results here allow us to make more specific predictions based on the ideal adapter, in two ways.

First, the informativity of talker identity is a measure of the variability across talkers. When talker identity is highly informative, there's more variability across talkers, and the ideal adapter predicts that prior experience with other talkers will be less relevant, resulting in faster and more complete adaptation to an unfamiliar talker. We found here that talker identity is less informative about VOT distributions than it is for vowel formant distributions. Hence, the ideal adapter predicts that listeners will adapt to talker-specific VOT distributions more slowly, and be more constrained by prior experience 
with other talkers. The first prediction is borne out by Kraljic and Samuel (2007), who compared recalibration of a voicing contrast with a fricative place contrast. It's also borne out indirectly by the modeling work in Chapters 2 and 4 , which found that the effective prior sample size for $/ \mathrm{b} /-/ \mathrm{d} /$ (which, like vowels, is primarily cued by formant frequencies) is much lower than for /b/-/p/ (cued by VOT).

Second, the informativity of higher-level grouping variables is linked to generalization across talkers: if two talkers are from groups that tend to differ, listeners should treat them separately and not generalize from experience with one talker to the other. Likewise, if two talkers are from the same group, listeners should generalize. We found that talker sex is informative for vowel formant distributions, but not for VOT, which means that listeners should generalize from a male to a female talker (and vice-versa) for a voicing contrast, but not for a vowel contrast. Listeners do, in fact, tend to generalize voicing recalibration across talkers of different sexes (Kraljic \& Samuel, 2006; Kraljic \& Samuel, 2007). While there's (to our knowledge) no data on cross-talker generalization for vowel recalibration, listeners tend not to generalize across talkers for fricative recalibration (Eisner \& McQueen, 2005; Kraljic \& Samuel, 2007), which (like vowels) are cued by spectral cues that vary across talkers and by gender (Newman et al., 2001; Jongman et al., 2000; McMurray \& Jongman, 2011).

There is also evidence for the prediction of generalization within informative groups. In the absence of evidence that two talkers from the same group (e.g. two males) produce a contrast differently, experience with one provides an informative starting point for comprehending (and adapting to) the other. Along these lines, van der Zande et al. (2014) found that listeners generalize from experience with one male talker's pronunciation of a $/ b /-/ d /$ contrast to another, unfamiliar male. 
Finally, it's important to point out that these predictions are best thought of as biases that might be overcome with enough of the right kind of evidence (Kleinschmidt \& Jaeger, 2015). For instance, listeners can overcome their bias to generalize experience with VOT and learn talker-specific VOT distributions, but it requires hundreds of observations from talkers who produce very different VOT distributions (C. M. Munson, 2011). Likewise, listeners will generalize recalibration of a fricative contrast from a female to a male talker given the right kind of test stimuli (Reinisch \& Holt, 2014).

\subsubsection{Sociolinguistic inference}

Our approach characterizes socio-linguistic judgments-like linguistic judgments-as probabilistic inference. ${ }^{13}$ In this view, both social and linguistic judgments rely on knowledge of how different underlying categories-social and linguistic-are probabilistically realized as distributions of observable cues. Just like each vowel (for instance) is realized as a distribution of F1 and F2 values, each dialect is also realized as an F1xF2 distribution (along with many other cues). When a listener hears a talker produce particular cue values, they can use knowledge of these distributions to compare how well each possible underlying social variable explains the speech they've observed. We find that this kind of model can classify a talker's dialect at roughly the same accuracy $(10-40 \%)$ as human listeners in a forced-choice task based on sentences spoken by the same talkers (Clopper \& Pisoni, 2006).

The idea of socio-linguistic judgments of inference fits naturally within the ideal adapter framework, which holds that listeners are simultaneously making at least three kinds of inferences in the normal course of speech perception:

\footnotetext{
${ }^{13}$ It is ultimately an empirical question whether this perspective is productive in understanding how listeners process language. At the very least, we hope that the formal and computational tools are helpful in formulating and test precise hypotheses about linguistic and socioindexical judgments.
} 
1. What a talker is saying

2. How that talker says things

3. Who that talker is, in relation to other talkers

The third level of inference is essential for talker-invariant speech perception: knowing who a talker is allows listeners to take advantage of their prior experience with other, similar talkers (Kleinschmidt \& Jaeger, 2015). Of course, listeners likely also want to know who a talker is for reasons that have nothing to do with accurate speech recognition per se (for similar points, see Babel \& Benjamin Munson, 2014; Pardo \& Remez, 2006; Foulkes \& Hay, 2015; Eckert, 2012a; Labov, 1972). To the extent that a talker's way of realizing linguistic variables says anything about who they are their speech is informative about their identity, at the same time as their identity is informative about their speech. Thus both sociolingusitic and psycholinguistic considerations lead to the idea that social inferences may well be inextricable from linguistic inferences.

Realizing that socio-linguistic judgments can be treated as a kind of inference is a potentially powerful idea, but it is important to realize that it is not, per se, a complete model of socio-linguistic judgments. Rather, it is a framework for developing such a model. In this view, the particular inferences that a listener would draw based on particular linguistic input depends not only on the distributions of cues in the world but just as much on the listener's own, internal model of how social variables relate to each other. Or, as it's more commonly put, a listener's stereotypes or ideologies about language use and social identity.

Careful sociolinguistic work is required to tease these factors out. One example comes from Levon (2014). He finds that when UK listeners hear a male talker with high /s/ spectral center of gravity (COG), they infer that the talker is a gay man. But when they hear a male talker with high /s/ spectral COG 
and TH-fronting (i.e., /f/ for / TH/), they judge the talker to be a working class straight man. That is, the inference that the talker is working class blocks the inference that he is gay. These sorts of effects are perfectly compatible with an inference-based perspective, but they depend on the specific contents of the listeners internal model of how social variables are related to each other and to observable cues (for examples of similar blocking effects in other domains, see Jacobs \& Kruschke, 2010; and in speech perception, see Kraljic, Samuel, \& Brennan, 2008; Kraljic \& Samuel, 2011; Liu \& Jaeger, 2016). Such internal models are not directly derivable from production data like we analyze here, but rather require probing a listener's subjective, implicit beliefs (as in the previous chapter).

\subsubsection{A lower bound}

Finally, it is important to note that our results here constitute a lower bound on the informativity or utility of different levels of socio-indexical grouping. ${ }^{14}$ We model cue distributions for a particular group as a single normal distribution over observed cue values. In reality, a hierarchical model is more appropriate, since different levels of grouping can nest within each other. For instance, each dialect group is likely better modeled as a mixture of talker-specific distributions, which each exhibit dialect features to a varying degree. This is especially important for adaptation to an unfamiliar talker, since a group-level distribution conflates within and between talker variation, both of which have separate roles to play in belief updating.

The approach to group-level modeling that we take here is roughly equivalent to the posterior predictive distribution of a fully hierarchical model, which

\footnotetext{
${ }^{14}$ Even above and beyond the limitations imposed by unequal numbers of talkers in each group, which necessitates subsampling talkers in the larger groups in order to meaningfully compare accuracy.
} 
integrates over lower levels of grouping to provide a single distribution of cues given the group (and phonetic category). This corresponds to the best guess a listener would have before hearing anything from an unfamiliar talker, if the only information they had about that talker was their group membership. As the listener hears more cue values from the talker, the hierarchical nature of grouping structure becomes more important and can provide (in principle) a significant advantage over what we measured here. But modeling this process is quite a bit more complicated and we leave it for future work. Nevertheless, modeling each category as a single, "flat" distribution per group may well prove a useful approximation, or even a boundedly-rational model of how listeners take advantage of different levels of grouping structure (and similar approaches have been used in, e.g., motor control Körding, Beierholm, et al., 2007).

\subsection{Conclusion}

Socio-linguistic variables like age, sex, and regional origin have been identified by sociolinguistics as factors that systematically affect the realization of linguistic categories. Using an ideal observer framework, we quantified the extent to which a range of these variables are informative about the distributions of acoustic cues corresponding to linguistic categories, useful for recognizing those categories, and can themselves be inferred from unlabeled cues. Our results show that the utility and informativity of a particular socio-indexical variable are closely related but not identical, while inferability is distinct. Moreover, we demonstrate how this method for quantifying these factors allows them to be compared across phonetic categories as well as cues/contrasts (VOT vs. F1xF2).

Together, these results show that the idea of inference under uncertainty, when applied to speech perception, provides a unifying perspective on both 
linguistic and socio-linguistic perception. This perspective leads to conceptual and computational tools for addressing questions that are of interest to psycholinguistics and sociolinguistics, as well as developing new bridges between the two. 


\section{Conclusion}

Human speech perception is a remarkable feat of perceptual inference. Despite massive variability in the way that linguistic units are realized by acoustic signals-both within and across talkers-listeners manage to reliably infer talkers' intended messages. The apparent ease by which this is accomplished belies the substantial computational complexity of the problem, and goes to show how well the human speech perception system is shaped byand hence matched to-the challenges presented by speech. The work presented in this thesis develops a theoretical and computational framework-the ideal adapter-for understanding one of the most long-standing problems in research on speech perception: how listeners cope with cross-talker variability.

This framework starts from the idea that speech perception is a problem of inference under uncertainty about the phonetic category (or other linguistic unit) that a talker intended to produce (e.g., Clayards et al., 2008; Feldman, Griffiths, \& Morgan, 2009; Norris \& McQueen, 2008). The ideal adapter extends these models by treating speech perception as a problem of inference under uncertainty at multiple levels. Robust speech perception requires that listeners continuously draw inferences not only about what the talker is trying to communicate, but also about the cue-category mappings that the talker is using (i.e., the talker's generative model). Moreover, in order to determine what pre- 
vious experience is relevant in making these inferences, and how relevant it is, these inferences in turn depend on higher-level inferences about who the talker is. This ranges from specific talker identity-supporting recognition of familiar talkers-to more general groups like gender, accent, or dialect-supporting generalization across similar talkers. The proposed multi-level inference solution can capture a variety of otherwise puzzling properties of speech adaptation

More importantly, the ideal adapter provides a guiding framework and a set of theoretical and computational tools for future research on speech perception, adaptation, and generalization. The ideal adapter specifically highlights listeners' beliefs about how talkers vary in the cue distributions they produce as critically important for understanding how listeners cope with that variability. According to the ideal adapter, these beliefs are used to guide and constrain adaptation to unfamiliar talkers, and are learned from experience with actual variation across talkers. Later chapters take first steps at understanding the constraints on rapid adaptation to unfamiliar talkers and quantifying the structure of variability across talkers. Both of these are underexplored areas which the ideal adapter highlights as central to understanding human speech perception.

Finally, the challenges posed by variability are not unique to speech perception, but rather general to the problem of effective perception and action in a variable world. This problem has been explored in the context of motor control (e.g., Körding, Beierholm, et al., 2007) and reinforcement learning (e.g., Cho et al., 2002; Gallistel et al., 2001), where it is typically cast as a problem of detecting changes in the statistics of the local environment (change detection). Our proposal highlights the fact that in a world where substantial parts of cross-situation variability are not random, but rather structured, simply detecting changes is not enough. Rather, learners can benefit from inferring the 
underlying structure to cross-situation variation, in order to recognize familiar situations and generalize to similar situations. In speech perception, the major source of variation across situations is the talker, but the same logic can be applied to other domains (Qian et al., 2012; Qian et al., 2015). The ideal adapter highlights the potential of speech perception to serve as a model organism for understanding perception in a variable-but structured-world, and suggests that superficially unrelated phenomena from non-linguistic perceptual/motor domains might be informative about language processing and acquisition and vice-versa. 


\section{References}

Ainsworth, W. A. (1977). Mechanisms of selective feature adaptation. Perception $\mathcal{E}$ Psychophysics, 21(4), 365-370. doi:10.3758/BF03199488

Ajmera, J., McCowan, I., \& Bourlard, H. (2004). Robust speaker change detection. Signal Processing Letters, IEEE, 11(8), 649-651.

Allen, J. S. \& Miller, J. L. (2004). Listener sensitivity to individual talker differences in voice-onset-time. The Journal of the Acoustical Society of America, 115(6), 3171. doi:10.1121/1.1701898

Allen, J. S., Miller, J. L., \& DeSteno, D. (2003). Individual talker differences in voice-onset-time. The Journal of the Acoustical Society of America, 113(1), 544. doi:10.1121/1.1528172

Anderson, J. R. (1990). The adaptive character of thought. Studies in cognition. Hillsdale, NJ: Lawrence Erlbaum Associates.

Anderson, J. R. (1991). The adaptive nature of human categorization. Psychological Review, 98(3), 409-429. doi:10.1037//0033-295X.98.3.409

Andruski, J. E., Blumstein, S. E., \& Burton, M. (1994). The effect of subphonetic differences on lexical access. Cognition, 52(3), 163-87.

Ashby, F. G. \& Alfonso-Reese, L. A. (1995). Categorization as Probability Density Estimation. Journal of Mathematical Psychology, 39(2), 216-233. doi:10. 1006/jmps.1995.1021 
Aslin, R. N., Saffran, J. R., \& Newport, E. L. (1998). Computation of Conditional Probability Statistics by 8-Month-Old Infants. Psychological Science, 9(4), 321-324. doi:10.1111/1467-9280.00063

Atick, J. J. \& Redlich, A. N. (1990). Towards a Theory of Early Visual Processing. Neural Computation, 2(3), 308-320. doi:10.1162/neco.1990.2.3.308

Attneave, F. (1954). Some informational aspects of visual perception. Psychological Review, 61(3), 183-193. doi:10.1037/h0054663

Babel, M. \& Munson, B. [Benjamin]. (2014). Producing Socially Meaningful Linguistic Variation. In V. Ferreira, M. Goldrick, \& M. Miozzo (Eds.), The oxford handbook of language production (pp. 308-328). Oxford: Oxford University Press.

Baese-berk, M. M., Bradlow, A. R., \& Wright, B. A. (2013). Accent-independent adaptation to foreign accented speech. JASA Express Letters, 133(3), 174180. doi:10.1121/1.4789864

Baese-Berk, M. \& Goldrick, M. (2009). Mechanisms of interaction in speech production. Language and Cognitive Processes, 24(4), 527-554. doi:10.1080 / 01690960802299378

Bard, E. G., Shillcock, R. C., \& Altmann, G. T. M. (1988). The recognition of words after their acoustic offsets in spontaneous speech: Effects of subsequent context. Perception \& Psychophysics, 44(5), 395-408. doi:10.3758 / BF03210424

Barlow, H. P. \& Földiák, P. (1989). Adaptation and Decorrelation in the Cortex. In R. Durbin, C. Miall, \& G. J. Mitchison (Eds.), The computing neuron (1988, pp. 54-72). Workingham, England: Addison-Wesley.

Beck, J. M., Ma, W. J., Pitkow, X., Latham, P. E., \& Pouget, A. (2012). Not noisy, just wrong: the role of suboptimal inference in behavioral variability. Neuron, 74(1), 30-9. doi:10.1016/j.neuron.2012.03.016 
Beck, J. M., Pouget, A., \& Heller, K. (2012). Complex Inference in Neural Circuits with Probabilistic Population Codes and Topic Models. In P. Bartlett (Ed.), Advances in neural information processing systems 25 (pp. 3059-67). Red Hook, NY: Curran Associates, Inc.

Bejjanki, V. R., Beck, J. M., Lu, Z.-L., \& Pouget, A. (2011). Perceptual learning as improved probabilistic inference in early sensory areas. Nature Neuroscience, 14(5), 642-8. doi:10.1038/nn.2796

Bejjanki, V. R., Clayards, M. A., Knill, D. C., \& Aslin, R. N. (2011). Cue integration in categorical tasks: Insights from audio-visual speech perception. PLoS ONE, 6(5), e19812. doi:10.1371/journal.pone.0019812

Bertelson, P., Vroomen, J., \& de Gelder, B. (2003). Visual recalibration of auditory speech identification: a McGurk aftereffect. Psychological Science, 14(6), 592-597. doi:10.1046/j.0956-7976.2003.psci_1470.x

Blakemore, C. \& Campbell, F. W. (1969). On the existence of neurones in the human visual system selectively sensitive to the orientation and size of retinal images. The Journal of Physiology, 203(1), 237-260.

Botvinick, M. M. (2012). Hierarchical reinforcement learning and decision making. Current opinion in neurobiology, 22(6), 956-62. doi:10.1016/j.conb.2012. 05.008

Botvinick, M. M. \& Plaut, D. C. (2004). Doing without schema hierarchies: a recurrent connectionist approach to normal and impaired routine sequential action. Psychological Review, 111(2), 395-429. doi:10.1037 / 0033-295X. 111.2.395

Bouton, M. E. \& King, D. a. (1983). Contextual control of the extinction of conditioned fear: tests for the associative value of the context. Journal of experimental psychology. Animal behavior processes, 9(3), 248-65.

Bradlow, A. R. \& Bent, T. (2008). Perceptual adaptation to non-native speech. Cognition, 106(2), 707-29. doi:10.1016/j.cognition.2007.04.005 
Brady, T. F. \& Tenenbaum, J. B. (2013). A probabilistic model of visual working memory: Incorporating higher order regularities into working memory capacity estimates. Psychological review, 120(1), 85-109. doi:10.1037 / a0030779

Brenner, N., Bialek, W., \& de Ruyter Van Steveninck, R. R. (2000). Adaptive Rescaling Maximizes Information Transmission. Neuron, 26(3), 695-702. doi:10.1016/S0896-6273(00)81205-2

Bricker, P. D. \& Pruzansky, S. (1966). Effects of Stimulus Content and Duration on Talker Identification. The Journal of the Acoustical Society of America, 40(6), 1441. doi:10.1121/1.1910246

Bryant, J. S. (1978). Feature detection process in speech perception. Journal of experimental psychology. Human perception and performance, 4(4), 610-620. doi:10.1037/0096-1523.4.4.610

Chambers, K. E., Onishi, K. H., \& Fisher, C. (2010). A vowel is a vowel: generalizing newly learned phonotactic constraints to new contexts. Journal of Experimental Psychology: Learning, Memory, and Cognition, 36(3), 821-8. doi:10.1037/a0018991

Chang, F., Dell, G. S., \& Bock, K. (2006). Becoming syntactic. Psychological Review, 113(2), 234-72. doi:10.1037/0033-295X.113.2.234

Chen, S. \& Gopalakrishnan, P. (1998). Speaker, environment and channel change detection and clustering via the Bayesian Information Criterion. In Proc. darpa broadcast news transcription and understanding workshop (p. 8). Virginia, USA.

Cho, R. Y., Nystrom, L. E., Brown, E. T., Jones, A. J., Braver, T. S., Holmes, P. J., \& Cohen, J. D. (2002). Mechanisms underlying dependencies of performance on stimulus history in a two-alternative forced-choice task. Cognitive, Affective, \& Behavioral Neuroscience, 2(4), 283-299. doi:10.3758/CABN. 2.4.283 
Chodroff, E., Godfrey, J., Khudanpur, S., \& Wilson, C. (2015). Structured Variability in Acoustic Realization : A Corpus Study of Voice Onset Time in American English Stops. In The Scottish Consortium for ICPhS (Ed.), Proceedings of the 18th international congress of phonetic sciences. Glasgow, UK: the University of Glasgow.

Chopin, A. \& Mamassian, P. (2012). Predictive properties of visual adaptation. Current biology, 22(7), 622-6. doi:10.1016/j.cub.2012.02.021

Clark, A. (2013). Whatever next? Predictive brains, situated agents, and the future of cognitive science. Behavioral and Brain Sciences, 36(3), 181-204. doi:10.1017/S0140525X12000477

Clarke-Davidson, C. M. \& Garrett, M. F. (2004). Rapid adaptation to foreignaccented English. The Journal of the Acoustical Society of America, 116(6), 3647. doi:10.1121/1.1815131

Clarke-Davidson, C. M., Luce, P. A., \& Sawusch, J. R. (2008). Does perceptual learning in speech reflect changes in phonetic category representation or decision bias? Perception \& Psychophysics, 70(4), 604-618. doi:10.3758/PP. 70.4.604

Clayards, M. A., Tanenhaus, M. K., Aslin, R. N., \& Jacobs, R. a. (2008). Perception of speech reflects optimal use of probabilistic speech cues. Cognition, 108(3), 804-9. doi:10.1016/j.cognition.2008.04.004

Clopper, C. G. \& Pisoni, D. B. (2006). Effects of region of origin and geographic mobility on perceptual dialect categorization. Language variation and change, 18(2), 193-221. doi:10.1017/S0954394506060091

Clopper, C. G. \& Pisoni, D. B. (2007). Free classification of regional dialects of American English. Journal of Phonetics, 35(3), 421-438. doi:10.1016/j.wocn. 2006.06.001

Clopper, C. G., Pisoni, D. B., \& de Jong, K. J. (2005). Acoustic characteristics of the vowel systems of six regional varieties of American English. The 
Journal of the Acoustical Society of America, 118(3), 1661. doi:10.1121 / 1. 2000774

Cole, R. A. \& Cooper, W. E. (1977). Properties of friction analyzers for [j]. The Journal of the Acoustical Society of America, 62(1), 177. doi:10.1121/1.381479

Cole, R. A., Cooper, W. E., Singer, J., \& Allard, F. (1975). Selective adaptation of English consonants using real speech. Perception \& Psychophysics, 18(3), 227-244. doi:10.3758/BF03205973

Connine, C. M., Blasko, D. G., \& Hall, M. (1991). Effects of subsequent sentence context in auditory word recognition: Temporal and linguistic constrainst. Journal of Memory and Language, 30(2), 234-250. doi:10.1016/0749-596X(91) 90005-5

Courville, A. C., Daw, N. D., \& Touretzky, D. S. (2006). Bayesian theories of conditioning in a changing world. Trends in cognitive sciences, 10(7), 294300. doi:10.1016/j.tics.2006.05.004

Creel, S. C., Aslin, R. N., \& Tanenhaus, M. K. (2008). Heeding the voice of experience: the role of talker variation in lexical access. Cognition, 106(2), 63364. doi:10.1016/j.cognition.2007.03.013

Creel, S. C. \& Bregman, M. R. (2011). How Talker Identity Relates to Language Processing. Language and Linguistics Compass, 5(5), 190-204. doi:10.1111/j. 1749-818X.2011.00276.x

Creel, S. C. \& Tumlin, M. a. (2011). On-line acoustic and semantic interpretation of talker information. Journal of Memory and Language, 65(3), 264-285. doi:10.1016/j.jml.2011.06.005

Croft, W., Bhattacharya, T., Kleinschmidt, D. F., Smith, D. E., \& Jaeger, T. F. (2011). Greenbergian universals, diachrony, and statistical analyses. Linguistic Typology, 15(2), 433-453. doi:10.1515/LITY.2011.029

Cutler, A., Eisner, F., McQueen, J. M., \& Norris, D. (2010). How abstract phonemic categories are necessary for coping with speaker-related variation. 
In C. Fougeron, B. Kühnert, M. D'Imperio, \& N. Vallée (Eds.), Laboratory phonology 10 (pp. 91-111). Berlin: De Gruyter Mouton.

Dahan, D. (2010). The Time Course of Interpretation in Speech Comprehension. Current Directions in Psychological Science, 19(2), 121-126. doi:10.1177 / 0963721410364726

Dahan, D., Drucker, S. J., \& Scarborough, R. a. (2008). Talker adaptation in speech perception: adjusting the signal or the representations? Cognition, 108(3), 710-8. doi:10.1016/j.cognition.2008.06.003

Dahan, D., Magnuson, J. S. [James S.], Tanenhaus, M. K., \& Hogan, E. M. (2001). Subcategorical mismatches and the time course of lexical access: Evidence for lexical competition. Language and Cognitive Processes, 16(5-6), 507-534. doi:10.1080/01690960143000074

Davis, M. H., Johnsrude, I. S., Hervais-Adelman, A., Taylor, K., \& McGettigan, C. (2005). Lexical information drives perceptual learning of distorted speech: evidence from the comprehension of noise-vocoded sentences. Journal of Experimental Psychology: General, 134(2), 222-41. doi:10.1037 / 0096-3445.134.2.222

Dayan, P. \& Niv, Y. (2008). Reinforcement learning: the good, the bad and the ugly. Current opinion in neurobiology, 18(2), 185-96. doi:10.1016/ j.conb. 2008.08.003

Delattre, P. C., Liberman, A. M., \& Cooper, F. S. (1955). Acoustic Loci and Transitional Cues for Consonants. The Journal of the Acoustical Society of America, 27(4), 769. doi:10.1121/1.1908024

Dell, G. S. \& Chang, F. (2014). The P-chain: relating sentence production and its disorders to comprehension and acquisition. Philosophical transactions of the Royal Society of London. Series B, Biological sciences, 369(1634), 20120394. doi:10.1098/rstb.2012.0394 
Dell, G. S., Reed, K. D., Adams, D. R., \& Meyer, A. S. (2000). Speech errors, phonotactic constraints, and implicit learning: A study of the role of experience in language production. Journal of Experimental Psychology: Learning, Memory, and Cognition, 26(6), 1355-1367. doi:10.1037/0278-7393.26.6. 1355

Dell, G. S. \& Warker, J. A. (2004). The tongue slips into (recently learned) patterns. In H. Quene \& V. van Heuven (Eds.), On speech and language: studies for sieb g. nooteboom (1, pp. 45-56). Utrecht: Netherlands Graduate School of Linguistics.

Demberg, V. \& Keller, F. (2008). Data from eye-tracking corpora as evidence for theories of syntactic processing complexity. Cognition, 109(2), 193-210. doi:10.1016/j.cognition.2008.07.008

Dillon, B., Dunbar, E., \& Idsardi, W. (2013). A single-stage approach to learning phonological categories: insights from Inuktitut. Cognitive science, 37(2), 344-77. doi:10.1111/cogs.12008

Drager, K. (2010). Sociophonetic Variation in Speech Perception. Language and Linguistics Compass, 4(7), 473-480. doi:10.1111/j.1749-818X.2010.00210.x

Dragoi, V., Sharma, J., \& Sur, M. (2000). Adaptation-induced plasticity of orientation tuning in adult visual cortex. Neuron, 28(1), 287-98.

Eckert, P. (1989). Jocks and Burnouts. New York: Teachers College Press.

Eckert, P. (2010). Affect, sound symbolism, and variation. University of Pennsylvania Working Papers in Linguistics, 15(2 Selected Papers from NWAV 37), $70-80$.

Eckert, P. (2012a). Three Waves of Variation Study: The Emergence of Meaning in the Study of Sociolinguistic Variation. Annual Review of Anthropology, 41, 87-100. doi:10.1146/annurev-anthro-092611-145828 
Eckert, P. (2012b). Three waves of variation study: The emergence of meaning in the study of sociolinguistic variation. Annual Review of Anthropology, 41(June), 87-100. doi:10.1146/annurev-anthro-092611-145828

Eckert, P. \& McConnell-Ginet, S. (2013). Language and Gender (2nd). Cambridge University Press.

Eimas, P. D. \& Corbit, J. D. (1973). Selective adaptation of linguistic feature detectors. Cognitive Psychology, 4(1), 99-109. doi:10.1016/0010-0285(73) 90006-6

Eisner, F. \& McQueen, J. M. (2005). The specificity of perceptual learning in speech processing. Perception \& Psychophysics, 67(2), 224-38.

Eisner, F. \& McQueen, J. M. (2006). Perceptual learning in speech: Stability over time. The Journal of the Acoustical Society of America, 119(4), 1950-3. doi:10. $1121 / 1.2178721$

Elman, J. L. (1990). Finding structure in time. Cognitive Science, 14, 179-211.

Ernestus, M. (2014). Acoustic reduction and the roles of abstractions and exemplars in speech processing. Lingua, 142, 27-41. doi:10.1016/j.lingua.2012. 12.006

Ernst, M. O. \& Banks, M. S. (2002). Humans integrate visual and haptic information in a statistically optimal fashion. Nature, 415(6870), 429-33. doi:10. $1038 / 415429 a$

Ernst, M. O. \& Bülthoff, H. H. (2004). Merging the senses into a robust percept. Trends in Cognitive Sciences, 8(4), 162-9. doi:10.1016/j.tics.2004.02.002

Fairhall, A. L., Lewen, G. D., Bialek, W., \& de Ruyter Van Steveninck, R. R. (2001). Efficiency and ambiguity in an adaptive neural code. Nature, 412(6849), 787-92. doi:10.1038/35090500

Farmer, T. A., Brown, M., \& Tanenhaus, M. K. (2013). Prediction, explanation, and the role of generative models in language processing. Behavioral and Brain Sciences, 36(3), 211-2. doi:10.1017/S0140525X12002312 
Feldman, N. H., Griffiths, T. L., Goldwater, S., \& Morgan, J. L. (2013). A role for the developing lexicon in phonetic category acquisition. Psychological Review, 120(4), 751-778. doi:10.1037/a0034245

Feldman, N. H., Griffiths, T. L., \& Morgan, J. L. (2009). The influence of categories on perception: explaining the perceptual magnet effect as optimal statistical inference. Psychological Review, 116(4), 752-82. doi:10.1037/ a0017196

Feldman, N. H., Myers, E. B., White, K. S., Griffiths, T. L., \& Morgan, J. L. (2013). Word-level information influences phonetic learning in adults and infants. Cognition, 127(3), 427-438.

Field, D. J. (1987). Relations between the statistics of natural images and the response properties of cortical cells. Journal of the Optical Society of America. A, Optics and image science, 4(12), 2379-2394. doi:10.1364/JOSAA.4.002379

Fine, A. B., Jaeger, T. F., Farmer, T. A., \& Qian, T. (2013). Rapid Expectation Adaptation during Syntactic Comprehension. PLoS ONE, 8(10), e77661. doi:10.1371/journal.pone.0077661

Fine, A. B., Qian, T., Jaeger, T. F., \& Jacobs, R. A. (2010). Is there syntactic adaptation in language comprehension. In J. Hale (Ed.), Proceedings of the 1st acl workshop on cognitive modeling and computational linguistics (pp. 18-26). Stroudsburg, PA: Association for Computational Linguistics.

Foulkes, P. \& Hay, J. (2015). The Emergence of Sociophonetic Structure, 292313.

Frank, M. C. \& Goodman, N. D. (2012). Predicting pragmatic reasoning in language games. Science, 336(6084), 998. doi:10.1126/science.1218633

Frank, M. C., Goodman, N. D., \& Tenenbaum, J. B. (2009). Using speakers' referential intentions to model early cross-situational word learning. Psychological science, 20(5), 578-85. doi:10.1111/j.1467-9280.2009.02335.x 
Frank, S. L. \& Bod, R. (2011). Insensitivity of the human sentence-processing system to hierarchical structure. Psychological science, 22(6), 829-34. doi:10. 1177/0956797611409589

Frisby, J. (1979). Seeing: Illusion, Brain and Mind. Oxford: Oxford University Press.

Friston, K. J. (2005). A theory of cortical responses. Philosophical Transactions of the Royal Society of London. Series B, Biological Sciences, 360(1456), 815-36. doi:10.1098/rstb.2005.1622

Froyen, V., Feldman, J., \& Singh, M. (2015). Bayesian Hierarchical Grouping : Perceptual Grouping as Mixture Estimation. Psychological Review, 122(4), 575-597.

Gallistel, C. R., Mark, T. A., King, A. P., \& Latham, P. E. (2001). The rat approximates an ideal detector of changes in rates of reward: Implications for the law of effect. Journal of Experimental Psychology: Animal Behavior Processes, 27(4), 354.

Gardner, J. L., Tokiyama, S. N., \& Lisberger, S. G. (2004). A population decoding framework for motion aftereffects on smooth pursuit eye movements. The Journal of neuroscience : the official journal of the Society for Neuroscience, 24(41), 9035-48. doi:10.1523/JNEUROSCI.0337-04.2004

Garnsey, S., Pearlmutter, N., Myers, E., \& Lotocky, M. (1997). The Contributions of Verb Bias and Plausibility to the Comprehension of Temporarily Ambiguous Sentences. Journal of Memory and Language, 37(37), 58-93.

Gauvain, J.-L. \& Lee, C.-H. [Chin-Hui]. (1994). Maximum a Posteriori Estimation for Multivariate Gaussian Mixture Observations of Markov Chains. IEEE Transactions on Speech and Audio Processing, 2(2), 291-298.

Gelman, A., Carlin, J. B., Stern, H. S., \& Rubin, D. B. (2003). Bayesian Data Analysis (Second). Chapman \& Hall/CRC Texts in Statistical Science. Taylor \& Francis. 
Gelman, A. \& Rubin, D. B. (1992). Inference from Iterative Simulation Using Multiple Sequences. Statistical Science, 7(4), 457-472. doi:10.1214 / ss / 1177011136

Gibson, B. R., Rogers, T. T., \& Zhu, X. (2013). Human semi-supervised learning. Topics in Cognitive Science, 5(1), 132-72. doi:10.1111/tops.12010

Goldinger, S. D. (1996). Words and voices: Episodic traces in spoken word identification and recognition memory. Journal of Experimental Psychology: Learning, Memory, and Cognition, 22(5), 1166-1183. doi:10 . 1037 / 0278 7393.22.5.1166

Goldinger, S. D. (1998). Echoes of echoes? An episodic theory of lexical access. Psychological Review, 105(2), 251-79.

Goldinger, S. D. (2007). A complementary-systems approach to abstract and episodic speech perception. In Icphs xvi (August, pp. 49-54).

Goldrick, M. (2004). Phonological features and phonotactic constraints in speech production. Journal of Memory and Language, 51(4), 586-603.

Goldrick, M., Vaughn, C., \& Murphy, A. (2013). The effects of lexical neighbors on stop consonant articulation. The Journal of the Acoustical Society of America, 134(2), EL172-7. doi:10.1121/1.4812821

Goldwater, S., Griffiths, T. L., \& Johnson, M. (2009). A Bayesian framework for word segmentation: exploring the effects of context. Cognition, 112(1), 2154. doi:10.1016/j.cognition.2009.03.008

Gómez, R. \& Gerken, L. (2000). Infant artificial language learning and language acquisition. Trends in cognitive sciences, 4(5), 178-186.

Goodman, N. D. \& Stuhlmüller, A. (2013). Knowledge and implicature: modeling language understanding as social cognition. Topics in cognitive science, 5(1), 173-84. doi:10.1111/tops.12007

Grabski, K., Tremblay, P., Gracco, V. L., Girin, L., \& Sato, M. (2013). A mediating role of the auditory dorsal pathway in selective adaptation to speech: A 
state-dependent transcranial magnetic stimulation study. Brain Research, 1515, 55-65. doi:10.1016/j.brainres.2013.03.024

Griffiths, T. L., Chater, N., Kemp, C., Perfors, A., \& Tenenbaum, J. B. (2010). Probabilistic models of cognition: exploring representations and inductive biases. Trends in Cognitive Sciences, 14(8), 357-64. doi:10.1016/j.tics. 2010.05.004

Griffiths, T. L., Sanborn, A. N., Canini, K. R., \& Navarro, D. J. (2008). Categorization as nonparametric Bayesian density estimation. The probabilistic mind: Prospects for Bayesian cognitive science, 303-328.

Griffiths, T. L., Vul, E., \& Sanborn, a. N. (2012). Bridging Levels of Analysis for Probabilistic Models of Cognition. Current Directions in Psychological Science, 21(4), 263-268. doi:10.1177/0963721412447619

Grodner, D. \& Sedivy, J. C. (2011). The effect of speaker-specific information on pragmatic inferences. In E. A. Gibson \& N. J. Pearlmutter (Eds.), The processing and acquisition of reference (pp. 239-272). Cambridge, MA: MIT Press.

Grosjean, F. (1985). The recognition of words after their acoustic offset: Evidence and implications. Perception E Psychophysics, 38(4), 299-310. doi:10. 3758/BF03207159

Guediche, S., Blumstein, S. E., Fiez, J. a., \& Holt, L. L. (2014). Speech perception under adverse conditions: insights from behavioral, computational, and neuroscience research. Frontiers in systems neuroscience, 7(January), 126. doi:10.3389/fnsys.2013.00126

Gutfreund, Y. (2012). Stimulus-specific adaptation, habituation and change detection in the gaze control system. Biological cybernetics. doi:10.1007 / s00422-012-0497-3

Gutnisky, D. a. \& Dragoi, V. (2008). Adaptive coding of visual information in neural populations. Nature, 452(7184), 220-4. doi:10.1038/nature06563 
Hagiwara, R. (1997). Dialect variation and formant frequency: The American English vowels revisited. The Journal of the Acoustical Society of America, 102(1), 655. doi:10.1121/1.419712

Hale, J. (2001). A probabilistic earley parser as a psycholinguistic model. In K. Knight (Ed.), Second meeting of the north american chapter of the association for computational linguistics on language technologies (Vol. 2, pp. 18). Stroudsburg, PA: Association for Computational Linguistics. doi:10. 3115/1073336.1073357

Hanulíková, A., van Alphen, P. M., van Goch, M. M., \& Weber, A. (2012). When one person's mistake is another's standard usage: the effect of foreign accent on syntactic processing. Journal of Cognitive Neuroscience, 24(4), 878887. doi:10.1162/jocn_a_00103

Harris, H., Gliksberg, M., \& Sagi, D. (2012). Generalized perceptual learning in the absence of sensory adaptation. Current biology, 22(19), 1813-7. doi:10. 1016/j.cub.2012.07.059

Hay, J. \& Drager, K. (2010). Stuffed toys and speech perception. Linguistics, 48(4), 865-892. doi:10.1515/ling.2010.027

Hay, J., Nolan, A., \& Drager, K. (2006). From fush to feesh: Exemplar priming in speech perception. The Linguistic Review, 23(3), 351-379. doi:10.1515/ TLR.2006.014

Hay, J., Warren, P., \& Drager, K. (2006). Factors influencing speech perception in the context of a merger-in-progress. Journal of Phonetics, 34(4), 458-484. doi:10.1016/j.wocn.2005.10.001

He, D., Kersten, D., \& Fang, F. (2012). Opposite modulation of high- and low-level visual aftereffects by perceptual grouping. Current biology : $C B$, 22(11), 1040-5. doi:10.1016/j.cub.2012.04.026 
Heald, S. L. M. \& Nusbaum, H. C. (2015). Variability in vowel production within and between days. PLOS ONE, 10(9). doi:10.1371/journal. pone. 0136791

Heid, S. \& Hawkins, S. (2000). An acoustical study of long-domain /r/ and /1/ coarticulation. In P. Hoole (Ed.), Proceedings of the 5 th seminar on speech production: models and data (pp. 77-80). Munich, Germany: SPS5.

Hillenbrand, J., Getty, L. A., Clark, M. J., \& Wheeler, K. (1995). Acoustic characteristics of American English vowels. The Journal of the Acoustical Society of America, 97(5.1), 3099-111.

Hinton, G. E. (2007). Learning multiple layers of representation. Trends in Cognitive Sciences, 11(10), 428-34. doi:10.1016/j.tics.2007.09.004

Holt, L. L. (2006). The mean matters: effects of statistically defined nonspeech spectral distributions on speech categorization. The Journal of the Acoustical Society of America, 120(5 Pt 1), 2801-17. doi:10.1121/1.2354071

House, A. S. (1953). The Influence of Consonant Environment upon the Secondary Acoustical Characteristics of Vowels. The Journal of the Acoustical Society of America, 25(1), 105. doi:10.1121/1.1906982

Huang, J. \& Holt, L. L. (2012). Listening for the norm: adaptive coding in speech categorization. Frontiers in Psychology, 3(February), 10. doi:10.3389/fpsyg. 2012.00010

Huang, Y. \& Rao, R. P. N. (2011). Predictive coding. Wiley Interdisciplinary Reviews: Cognitive Science, 2(5), 580-593. doi:10.1002/wcs.142

Idemaru, K. \& Holt, L. L. (2011). Word recognition reflects dimension-based statistical learning. Journal of Experimental Psychology: Human Perception and Performance, 37(6), 1939-56. doi:10.1037/a0025641

Jacobs, R. A. (2002). What determines visual cue reliability? Trends in Cognitive Sciences, 6(8), 345-350. 
Jacobs, R. A. \& Kruschke, J. K. (2010). Bayesian learning theory applied to human cognition. Wiley Interdisciplinary Reviews: Cognitive Science, n/a-n/a. doi:10.1002/wcs.80

Jaeger, T. F. (2008). Categorical Data Analysis: Away from ANOVAs (transformation or not) and towards Logit Mixed Models. Journal of memory and language, 59(4), 434-446. doi:10.1016/j.jml.2007.11.007

Jaeger, T. F. (2013). Production preferences cannot be understood without reference to communication. Frontiers in Psychology, 4(April), 230. doi:10.3389/ fpsyg.2013.00230

Jaeger, T. F. \& Ferreira, V. S. (2013). Seeking predictions from a predictive framework. Behavioral and Brain Sciences, 36(4), 359-60. doi:10. 1017 / S0140525X12002762

Jaeger, T. F., Graff, P., Croft, W., \& Pontillo, D. (2011). Mixed effect models for genetic and areal dependencies in linguistic typology. Linguistic Typology, 15(2). doi:10.1515/lity.2011.021

Jaeger, T. F. \& Snider, N. E. (2013). Alignment as a consequence of expectation adaptation: syntactic priming is affected by the prime's prediction error given both prior and recent experience. Cognition, 127(1), 57-83. doi:10. 1016/j.cognition.2012.10.013

Jaeger, T. F. \& Weatherholtz, K. (2016). What the Heck Is Salience? How Predictive Language Processing Contributes to Sociolinguistic Perception. Frontiers in Psychology, 7(August), 1-5. doi:10.3389/fpsyg.2016.01115

James, G., Witten, D., Tibshirani, R., \& Hastie, T. (2013). An Introduction to Statistical Learning with Applications in R. Book, 431. doi:10.1007/978-14614-7138-7

Johnson, K. (1997a). Speech perception without speaker normalization: An exemplar model. In Johnson \& Mullennix (Eds.), Talker variability in speech processing (pp. 145-165). San Diego: Academic Press. 
Johnson, K. (1997b). The auditory/perceptual basis for speech segmentation. OSU Working Papers in Linguistics. OSU Working Papers in Linguistics, 50, 101-113.

Johnson, K. (2005). Speaker normalization in speech perception. In D. B. Pisoni \& R. E. Remez (Eds.), The handbook of speech perception (Figure 1, pp. 363389). Oxford: Blackwell Publishers.

Johnson, K. (2006). Resonance in an exemplar-based lexicon: The emergence of social identity and phonology. Journal of Phonetics, 34(4), 485-499. doi:10. 1016/j.wocn.2005.08.004

Johnson, K. (2013). Factors that affect phonetic adaptation: Exemplar filters and sound change. In Talk presented at current issues and methods in speaker adaptation.

Johnson, K., Strand, E. A., \& D'Imperio, M. (1999). Auditory-visual integration of talker gender in vowel perception. Journal of Phonetics, 27(4), 359-384. doi:10.1006/jpho.1999.0100

Jongman, A., Wayland, R., \& Wong, S. (2000). Acoustic characteristics of English fricatives. The Journal of the Acoustical Society of America, 108(3), 1252. doi:10.1121/1.1288413

Kamide, Y. (2012). Learning individual talkers' structural preferences. Cognition, 124(1), 66-71. doi:10.1016/j.cognition.2012.03.001

Kaschak, M. P. \& Glenberg, A. M. (2004). This construction needs learned. Journal of Experimental Psychology: General, 133(3), 450-67. doi:10.1037 / 00963445.133.3.450

Kersten, D., Mamassian, P., \& Yuille, A. (2004). Object perception as Bayesian inference. Annual Review of Psychology, 55, 271-304. doi:10.1146/annurev. psych.55.090902.142005 
Klatt, D. H. (1980). Software for a cascade/paralell formant synthesizer. Journal of the Acoustical Society of America, 67(3), 971-995. doi:10.3758/BRM.42.3. 863

Kleinschmidt, D. F., Emberson, L. L., Doko, D., Edelman, S., \& Raizada, R. D. S. (2015). How the brain transforms its representational space to learn to solve a linearly inseparable task. Manuscript in preparation.

Kleinschmidt, D. F., Fine, A. B., \& Jaeger, T. F. (2012). A belief-updating model of adaptation and cue combination in syntactic comprehension. In N. Miyake, D. Peebles, \& R. P. Cooper (Eds.), Proceedings of the 34th annual conference of the cognitive science society (pp. 599-604). Austin, TX: Cognitive Science Society.

Kleinschmidt, D. F. \& Jaeger, T. F. (2011). A Bayesian belief updating model of phonetic recalibration and selective adaptation. In Proceedings of the 2 nd acl workshop on cognitive modeling and computational linguistics. Stroudsburg, PA: Association for Computational Linguistics.

Kleinschmidt, D. F. \& Jaeger, T. F. (2012). A continuum of phonetic adaptation: Evaluating an incremental belief-updating model of recalibration and selective adaptation. In N. Miyake, D. Peebles, \& R. P. Cooper (Eds.), Proceedings of the 34th annual conference of the cognitive science society (pp. 60510). Austin, TX: Cognitive Science Society.

Kleinschmidt, D. F. \& Jaeger, T. F. (2015). Robust speech perception: Recognize the familiar, generalize to the similar, and adapt to the novel. Psychological Review, 122(2), 148-203. doi:10.1037/a0038695

Kleinschmidt, D. F. \& Jaeger, T. F. (2016a). Re-examining selective adaptation: Fatiguing feature detectors, or distributional learning? Psychonomic Bulletin \& Review, 23(3), 678-691. doi:10.3758/s13423-015-0943-z

Kleinschmidt, D. F. \& Jaeger, T. F. (2016b). What do you expect from an unfamiliar talker? In J. Trueswell, A. Papafragou, D. Grodner, \& D. Mirman 
(Eds.), Proceedings of the 38th annual meeting of the cognitive science society. Austin, TX: Cognitive Science Society.

Kleinschmidt, D. F., Raizada, R., \& Jaeger, T. F. (2015). Supervised and unsupervised learning in phonetic adaptation. In R. Dale, C. Jennings, P. Maglio, T. Matlock, D. Noelle, A. Warlaumont, \& J. Yoshimi (Eds.), Proceedings of the 37th annual conference of the cognitive science society. Austin, TX: Cognitive Science Society.

Knill, D. C. \& Richards, W. (Eds.). (1996). Perception as Bayesian Inference. Cambridge, UK: Cambridge University Press.

Knill, D. C. \& Saunders, J. A. (2003). Do humans optimally integrate stereo and texture information for judgments of surface slant? Vision Research, 43(24), 2539-2558. doi:10.1016/S0042-6989(03)00458-9

Knudsen, E. I. (1998). Capacity for Plasticity in the Adult Owl Auditory System Expanded by Juvenile Experience. Science, 279(5356), 1531-1533. doi:10. 1126/science.279.5356.1531

Kohler, K. J. (1982). F0 in the production of lenis and fortis plosives. Phonetica, 39(4-5), 199-218. doi:10.1159/000261663

Kohn, A. (2007). Visual adaptation: physiology, mechanisms, and functional benefits. Journal of Neurophysiology, 97(5), 3155-64. doi:10.1152/jn.00086. 2007

Kohn, A. \& Movshon, J. A. (2004). Adaptation changes the direction tuning of macaque MT neurons. Nature Neuroscience, 7(7), 764-72. doi:10.1038 / nn1267

Körding, K. P., Beierholm, U., Ma, W. J., Quartz, S., Tenenbaum, J. B., \& Shams, L. (2007). Causal inference in multisensory perception. PLoS ONE, 2(9), e943. doi:10.1371/journal.pone.0000943 
Körding, K. P., Tenenbaum, J. B., \& Shadmehr, R. (2007). The dynamics of memory as a consequence of optimal adaptation to a changing body. Nature Neuroscience, 10(6), 779-86. doi:10.1038/nn1901

Kraljic, T. \& Samuel, A. G. (2005). Perceptual learning for speech: Is there a return to normal? Cognitive Psychology, 51(2), 141-78. doi:10.1016 / j. cogpsych.2005.05.001

Kraljic, T. \& Samuel, A. G. (2006). Generalization in perceptual learning for speech. Psychonomic Bulletin E Review, 13(2), 262-8.

Kraljic, T. \& Samuel, A. G. (2007). Perceptual adjustments to multiple speakers. Journal of Memory and Language, 56(1), 1-15. doi:10.1016/j.jml.2006.07.010

Kraljic, T. \& Samuel, A. G. (2011). Perceptual learning evidence for contextuallyspecific representations. Cognition, 121(3), 459-65. doi:10 . 1016 / j . cognition.2011.08.015

Kraljic, T., Samuel, A. G., \& Brennan, S. E. (2008). First impressions and last resorts: how listeners adjust to speaker variability. Psychological Science, 19(4), 332-8. doi:10.1111/j.1467-9280.2008.02090.x

Kronrod, Y., Coppess, E., \& Feldman, N. H. (2012). A Unified Model of Categorical Effects in Consonant and Vowel Perception. In N. Miyake, D. Peebles, \& R. P. Cooper (Eds.), Proceedings of the 34th annual conference of the cognitive science society (pp. 629-634). Austin, TX: Cognitive Science Society.

Kuhl, P. K. \& Miller, J. D. (1975). Speech perception by the chinchilla: Voicevoiceless distinction in alveolar plosive consonants. Science, 190(4209), 6972.

Kuperberg, G. R. \& Jaeger, T. F. (2016). What do we mean by prediction in language comprehension? Language Cognition $\mathcal{E}$ Neuroscience, 31(1), 32-59. doi:10.1080/23273798.2015.1102299

Kurumada, C., Brown, M., Bibyk, S., Pontillo, D. F., \& Tanenhaus, M. K. (2013). Incremental processing in the pragmatic interpretation of contrastive 
prosody. In M. Knauff, M. Pauen, N. Sebanz, \& I. Wachsmuth (Eds.), Proceedings of the 35th annual conference of the cognitive science society (pp. 846-51). Austin, TX: Cognitive Science Society.

Kurumada, C., Brown, M., Bibyk, S., Pontillo, D. F., \& Tanenhaus, M. K. (2014). Rapid adaptation in online pragmatic interpretation of contrastive prosody. In P. Bello, M. Guarini, M. McShane, \& B. Scassellati (Eds.), Proceedings of the 36th annual meeting of the cognitive science society (pp. 791796). Austin, TX: Cognitive Science Society.

Kurumada, C., Brown, M., \& Tanenhaus, M. K. (2012). Pragmatic interpretation of contrastive prosody : It looks like speech adaptation. In N. Miyake, D. Peebles, \& R. P. Cooper (Eds.), Proceedings of the 34th annual conference of the cognitive science society (pp. 647-652). Austin, TX: Cognitive Science Society.

Labov, W. (1972). Sociolinguistic Patterns. Conduct \& Communication Series. University of Pennsylvania Press.

Labov, W., Ash, S., \& Boberg, C. (2005). The Atlas of North American English. doi:10.1515/9783110206838

Laing, E. J. C., Liu, R., Lotto, A. J., \& Holt, L. L. (2012). Tuned with a Tune: Talker Normalization via General Auditory Processes. Frontiers in Psychology, 3(June), 203. doi:10.3389/fpsyg.2012.00203

Lancia, L. \& Winter, B. (2013). The interaction between competition, learning, and habituation dynamics in speech perception. Laboratory Phonology, 4(1), 221-258. doi:10.1515/lp-2013-0009

Leggetter, C. \& Woodland, P. (1995). Maximum likelihood linear regression for speaker adaptation of continuous density hidden Markov models. Computer Speech \& Language, 9(2), 171-185. doi:10.1006/csla.1995.0010 
Lev-Ari, S. \& Peperkamp, S. (2013). Low inhibitory skill leads to non-native perception and production in bilinguals' native language. Journal of Phonetics, 41(5), 320-331. doi:10.1016/j.wocn.2013.06.002

Levon, E. (2014). Categories, stereotypes, and the linguistic perception of sexuality. Language in Society, 43(5), 539-566. doi:10.1017/S0047404514000554

Levy, R. (2008a). A noisy-channel model of rational human sentence comprehension under uncertain input. In S. Padó (Ed.), Proceedings of the 2008 conference on empirical methods in natural language processing (October, pp. 234243). Stroudsburg, PA: Association for Computational Linguistics.

Levy, R. (2008b). Expectation-based syntactic comprehension. Cognition, 106(3), 1126-77. doi:10.1016/j.cognition.2007.05.006

Levy, R., Bicknell, K., Slattery, T., \& Rayner, K. (2009). Eye movement evidence that readers maintain and act on uncertainty about past linguistic input. Proceedings of the National Academy of Sciences of the United States of America, 106(50), 21086-90. doi:10.1073/pnas.0907664106

Lewicki, M. S. (2002). Efficient coding of natural sounds. Nature Neuroscience, 5(4), 356-63. doi:10.1038/nn831

Liberman, A. M., Cooper, F. S., Shankweiler, D. P., \& Studdert-Kennedy, M. (1967). Perception of the speech code. Psychological Review, 74(6), 431-61.

Linkenhoker, B. A., von der Ohe, C. G., \& Knudsen, E. I. (2005). Anatomical traces of juvenile learning in the auditory system of adult barn owls. $\mathrm{Na}$ ture neuroscience, 8(1), 93-8. doi:10.1038/nn1367

Lisker, L. \& Abramson, A. (1964). A cross-language sudy of voicing in initial stops: Acoustical measurements. Word, 20(3), 384-422.

Liu, L. \& Jaeger, T. F. (2016). Speech perception in the face of uncertainty. Manuscript in preparation. 
Lobanov, B. M. (1971). Classification of Russian Vowels Spoken by Different Speakers. The Journal of the Acoustical Society of America, 49(2B), 606. doi:10. $1121 / 1.1912396$

Ma, W. J., Beck, J. M., Latham, P. E., \& Pouget, A. (2006). Bayesian inference with probabilistic population codes. Nature Neuroscience, 9(11), 1432-8. doi:10. $1038 / \mathrm{nn} 1790$

MacDonald, M. C. (2013). How language production shapes language form and comprehension. Frontiers in Psychology, 4, 226. doi:10.3389 / fpsyg.2013. 00226

MacDonald, M. C., Pearlmutter, N. J., \& Seidenberg, M. S. (1994). Lexical nature of syntactic ambiguity resolution. Psychological Review, 101(4), 676-703.

MacKay, D. J. C. (2003). Information Theory, Inference, and Learning Algorithms (3rd ed.). Cambridge, UK: Cambridge University Press.

Magnuson, J. S. [James S] \& Nusbaum, H. C. (2007). Acoustic differences, listener expectations, and the perceptual accommodation of talker variability. Journal of Experimental Psychology: Human Perception and Performance, 33(2), 391-409. doi:10.1037/0096-1523.33.2.391

Mahon, B. Z., Costa, A., Peterson, R., Vargas, K. a., \& Caramazza, A. (2007). Lexical selection is not by competition: a reinterpretation of semantic interference and facilitation effects in the picture-word interference paradigm. Journal of experimental psychology. Learning, memory, and cognition, 33(3), 503-535. doi:10.1037/0278-7393.33.3.503

Marr, D. (1982). Vision: A computational investigation into the human representation and processing of visual information. New York, NY, USA: Henry Holt and Co., Inc.

Marslen-Wilson, W. \& Warren, P. (1994). Levels of perceptual representation and process in lexical access: Words, phonemes, and features. Psychological Review, 101(4), 653-675. doi:10.1037/0033-295X.101.4.653 
Massaro, D. W. (1987). Speech Perception by Ear and Eye: A Paradigm for Psychological Inquiry. Hillsdale, NJ: Erlbaum Associates.

Massaro, D. W. (2004). From multisensory integration to talking heads and language learning. In G. Calvert, C. Spence, \& B. E. Stein (Eds.), The handbook of multisensory processes (pp. 153-176). Cambridge, MA: MIT Press.

Maye, J., Aslin, R. N., \& Tanenhaus, M. K. (2008). The Weckud Wetch of the Wast: Lexical Adaptation to a Novel Accent. Cognitive Science, 32(3), 543562. doi:10.1080/03640210802035357

Maye, J., Werker, J. F., \& Gerken, L. (2002). Infant sensitivity to distributional information can affect phonetic discrimination. Cognition, 82(3), B101-11.

McClelland, J. L. \& Elman, J. L. (1986). The TRACE model of speech perception. Cognitive Psychology, 18(1), 1-86. doi:10.1016/0010-0285(86)90015-0

McGurk, H. \& MacDonald, J. (1976). Hearing lips and seeing voices. Nature, 264(5588), 746-748. doi:10.1038/264746a0

McLennan, C. T., Luce, P. A., \& Charles-Luce, J. (2003). Representation of lexical form. Journal of Experimental Psychology: Learning, Memory, and Cognition, 29(4), 539-553. doi:10.1037/0278-7393.29.4.539

McMurray, B., Aslin, R. N., \& Toscano, J. C. (2009). Statistical learning of phonetic categories: insights from a computational approach. Developmental Science, 12(3), 369-78. doi:10.1111/j.1467-7687.2009.00822.x

McMurray, B. \& Jongman, A. (2011). What information is necessary for speech categorization? Harnessing variability in the speech signal by integrating cues computed relative to expectations. Psychological Review, 118(2), 21946. doi:10.1037/a0022325

McMurray, B., Tanenhaus, M. K., \& Aslin, R. N. (2002). Gradient effects of within-category phonetic variation on lexical access. Cognition, 86(2), B3342. 
McQueen, J. M., Cutler, A., \& Norris, D. (2006). Phonological Abstraction in the Mental Lexicon. Cognitive Science, 30(6), 1113-1126. doi:10.1207 / s15516709cog0000_79

McQueen, J. M., Norris, D., \& Cutler, A. (1999). Lexical influence in phonetic decision making: Evidence from subcategorical mismatches. Journal of Experimental Psychology: Human Perception and Performance, 25(5), 1363-1389. doi:10.1037/ / 0096-1523.25.5.1363

Miller, J. L., Connine, C. M., Schermer, T. M., \& Kluender, K. R. (1983). A possible auditory basis for internal structure of phonetic categories. The Journal of the Acoustical Society of America, 73(6), 2124-33.

Mirman, D., McClelland, J. L., \& Holt, L. L. (2006). An interactive Hebbian account of lexically guided tuning of speech perception. Psychonomic bulletin E review, 13(6), 958-65.

Mitterer, H. \& Reinisch, E. (2013). No delays in application of perceptual learning in speech recognition: Evidence from eye tracking. Journal of Memory and Language, 69(4), 527-545. doi:10.1016/j.jml.2013.07.002

Mollon, J. (1974). After-effects and the brain. New Scientist, 479-482.

Moulin-Frier, C., Diard, J., Schwartz, J. L., \& Bessière, P. (2015). COSMO ("Communicating about Objects using Sensory-Motor Operations"): A Bayesian modeling framework for studying speech communication and the emergence of phonological systems. Journal of Phonetics, 53, 5-41. doi:10.1016/ j.wocn.2015.06.001

Munson, B. [B.]. (2007). The Acoustic Correlates of Perceived Masculinity, Perceived Femininity, and Perceived Sexual Orientation. Language and Speech, 50(1), 125-142. doi:10.1177/00238309070500010601

Munson, B. [Benjamin]. (2011). The influence of actual and imputed talker gender on fricative perception, revisited (L). The Journal of the Acoustical Society of America, 130(5), 2631-4. doi:10.1121/1.3641410 
Munson, C. M. (2011). Perceptual learning in speech reveals pathways of processing (Doctoral dissertation, University of Iowa).

Murphy, K. P. (2007). Conjugate Bayesian analysis of the Gaussian distribution. Technical report, University of British Columbia.

Neal, R. M. (2003). Slice Sampling. Annals of statistics, 31(3), 705-741.

Nearey, T. M. (1997). Speech perception as pattern recognition. The Journal of the Acoustical Society of America, 101(6), 3241-3254.

Nearey, T. M. \& Assman, P. F. (1986). Modeling the role of inherent spectral change in vowel identification. The Journal of the Acoustical Society of America, 80(5), 1297. doi:10.1121/1.394433

Newman, R. S., Clouse, S. A., \& Burnham, J. L. (2001). The perceptual consequences of within-talker variability in fricative production. The Journal of the Acoustical Society of America, 109(3), 1181-1196. doi:10.1121/1.1348009

Niedzielski, N. (1999). The Effect of Social Information on the Perception of Sociolinguistic Variables. Journal of Language and Social Psychology, 18(1), 62-85. doi:10.1177/0261927X99018001005

Norris, D. (1994). Shortlist: a connectionist model of continuous speech recognition. Cognition, 52(3), 189-234. doi:10.1016/0010-0277(94)90043-4

Norris, D. \& McQueen, J. M. (2008). Shortlist B: A Bayesian model of continuous speech recognition. Psychological Review, 115(2), 357-95. doi:10.1037/ 0033-295X.115.2.357

Norris, D., McQueen, J. M., \& Cutler, A. (2000). Merging information in speech recognition: feedback is never necessary. The Behavioral and brain sciences, 23(3), 299-325, 299-325.

Norris, D., McQueen, J. M., \& Cutler, A. (2003). Perceptual learning in speech. Cognitive Psychology, 47(2), 204-238. doi:10.1016/S0010-0285(03)00006-9

Nygaard, L. C. \& Pisoni, D. B. (1998). Talker-specific learning in speech perception. Perception \& Psychophysics, 60(3), 355-76. 
Oden, G. C. \& Massaro, D. W. (1978). Integration of featural information in speech perception. Psychological Review, 85(3), 172-191. doi:10.1037/0033295X.85.3.172

O'Donnell, T. J., Snedeker, J., Tenenbaum, J. B., \& Goodman, N. D. (2011). Productivity and reuse in language. In L. Carlson, C. Hoelscher, \& T. F. Shipley (Eds.), Proceedings of the 33rd annual conference of the cognitive science society (pp. 1613-8). Austin, TX: Cognitive Science Society.

Olshausen, B. A. \& Field, D. J. (1996). Emergence of simple-cell receptive field properties by learning a sparse code for natural images. Nature, 381(6583), 607-9. doi:10.1038/381607a0

Oppenheim, G. M., Dell, G. S., \& Schwartz, M. F. (2010). The dark side of incremental learning: A model of cumulative semantic interference during lexical access in speech production. Cognition, 114(2), 227-252. doi:10.1016/ j.cognition.2009.09.007

Orhan, A. E. \& Jacobs, R. A. (2011). A Nonparametric Bayesian Model of Visual Short-Term Memory. In L. Carlson, C. Hoelscher, \& T. F. Shipley (Eds.), Proceedings of the 33rd annual conference of the cognitive science society (pp. 2451-2456). Austin, TX: Cognitive Science Society.

Pajak, B., Fine, A. B., Kleinschmidt, D. F., \& Jaeger, T. F. (2016). Learning Additional Languages as Hierarchical Probabilistic Inference: Insights From First Language Processing. Language Learning, e-pub ahea. doi:10.1111 / lang. 12168

Pajak, B. \& Levy, R. (2011). Phonological Generalization from Distributional Evidence. In L. Carlson, C. Hoelscher, \& T. F. Shipley (Eds.), Proceedings of the 33rd annual conference of the cognitive science society (pp. 2673-8). Austin, TX: Cognitive Science Society. 
Palmeri, T. J., Goldinger, S. D., \& Pisoni, D. B. (1993). Episodic encoding of voice attributes and recognition memory for spoken words. Journal of Experimental Psychology: Learning, Memory, and Cognition, 19(2), 309-28.

Pardo, J. S. \& Remez, R. E. (2006). The Perception of Speech. In M. Traxler \& M. A. Gernsbacher (Eds.), The handbook of psycholinguisitcs (2nd, pp. 201248). New York.

Perfors, A., Tenenbaum, J. B., \& Regier, T. (2011). The learnability of abstract syntactic principles. Cognition, 118(3), 306-338. doi:10.1016/j.cognition. 2010.11.001

Peterson, G. E. \& Barney, H. L. (1952). Control Methods Used in a Study of the Vowels. The Journal of the Acoustical Society of America, 24(2), 175-184. doi:10.1121/1.1906875

Pickering, M. J. \& Garrod, S. (2013). An integrated theory of language production and comprehension. Behavioral and Brain Sciences, 36(4), 329-47. doi:10.1017/S0140525X12001495

Pierrehumbert, J. B. (2001). Exemplar dynamics: Word frequency, lenition and contrast. In J. Bybee \& P. Hopper (Eds.), Frequency effects and the emergence of lexical structure (Hooper 1976, pp. 137-157). Amsterdam: John Benjamins.

Pierrehumbert, J. B. (2002). Word-specific phonetics. Laboratory Phonology, 1-24.

Pierrehumbert, J. B. (2003). Phonetic diversity, statistical learning, and acquisition of phonology. Language and Speech, 46(Pt 2-3), 115-54.

Pierrehumbert, J. B. (2006). The next toolkit. Journal of Phonetics, 34(4), 516-530. doi:10.1016/j.wocn.2006.06.003

Pisoni, D. B. \& Levi, S. V. (2007). Representations and representational specificity in speech perception and spoken word recognition. In M. G. Gaskell (Ed.), The oxford handbook of psycholinguistics (pp. 3-18). Oxford: Oxford University Press. 
Pisoni, D. B. \& Tash, J. (1974). Reaction times to comparisons within and across phonetic categories. Perception \& Psychophysics, 15(2), 285-290.

Pitt, M. A., Dilley, L. C., Johnson, K., Kiesling, S., Raymond, W., Hume, E., \& Fosler-Lussier, E. (2007). Buckeye Corpus of Conversational Speech (2nd release). Columbus, OH: Department of Psychlogy, Ohio State University.

Podesva, R. J. (2007). Phonation type as a stylistic variable: The use of falsetto in constructing a persona. Journal of Sociolinguistics, 11(4), 478-504. doi:10. $1111 / \mathrm{j} .1467-9841.2007 .00334 . x$

Podesva, R. J., Roberts, S. J., \& Campbell-Kibler, K. (2001). Sharing resources and indexing meanings in the production of gay styles. In K. CampbellKibler, R. J. Podesva, S. J. Roberts, \& A. Wong (Eds.), Language and sexuality: contesting meaning in theory and practice (pp. 175-189). CSLI Publications.

Qian, T., Jaeger, T. F., \& Aslin, R. N. (2012). Learning to represent a multicontext environment: more than detecting changes. Frontiers in Psychology, 3(July), 228. doi:10.3389/fpsyg.2012.00228

Qian, T., Jaeger, T. F., \& Aslin, R. N. (2015). Implicit Learning of Bundles of Statistical Patterns in an Incremental Task. Manuscript submitted for publication.

Rao, R. P. N. \& Ballard, D. H. (1999). Predictive coding in the visual cortex: a functional interpretation of some extra-classical receptive-field effects. Nature Neuroscience, 2(1), 79-87. doi:10.1038/4580

Reinisch, E. \& Holt, L. L. (2014). Lexically guided phonetic retuning of foreignaccented speech and its generalization. Journal of experimental psychology. Human perception and performance, 40(2), 539-55. doi:10.1037 / a0034409. arXiv: NIHMS150003 
Remez, R. E. (1979). Adaptation of the category boundary between speech and nonspeech: A case against feature detectors. Cognitive Psychology, 11(1), 38-57. doi:10.1016/0010-0285(79)90003-3

Remez, R. E. (1987). Neural models of speech perception: $\{A\}$ case history. In S. Harnad (Ed.), Categorical perception (Chap. 6, pp. 199-224). New York: Cambridge University Press.

Remez, R. E., Fellowes, J. M., \& Rubin, P. E. (1997). Talker identification based on phonetic information. Journal of Experimental Psychology: Human Perception and Performance, 23(3), 651-666. doi:10.1037/0096-1523.23.3.651

Remez, R. E., Rubin, P. E., Pisoni, D. B., \& Carrell, T. (1981). Speech perception without traditional speech cues. Science, 212(4497), 947.

Richter, C., Feldman, N. H., Salgado, H., \& Jansen, A. (2016). A Framework for Evaluating Speech Representations. In J. Trueswell, A. Papafragou, D. Grodner, \& D. Mirman (Eds.), Proceedings of the 38th annual meeting of the cognitive science society. Austin, TX: Cognitive Science Society.

Roberts, M. \& Summerfield, Q. (1981). Audiovisual presentation demonstrates that selective adaptation in speech perception is purely auditory. Perception \& Psychophysics, 30(4), 309-14.

Saffran, J. R., Aslin, R. N., \& Newport, E. L. (1996). Statistical learning by 8month-old infants. Science, 274(5294), 1926-8.

Saffran, J. R., Newport, E. L., \& Aslin, R. N. (1996). Word Segmentation: The Role of Distributional Cues. Journal of Memory and Language, 35(4), 606621. doi:10.1006/jmla.1996.0032

Saldaña, H. M. \& Rosenblum, L. D. (1994). Selective adaptation in speech perception using a compelling audiovisual adaptor. The Journal of the Acoustical Society of America, 95(6), 3658-61. 
Salverda, A. P., Kleinschmidt, D. F., \& Tanenhaus, M. K. (2014). Immediate effects of anticipatory coarticulation in spoken-word recognition. Journal of Memory and Language, 71(1), 145-163. doi:10.1016/j.jml.2013.11.002

Samuel, A. G. (1986). Red herring detectors and speech perception: in defense of selective adaptation. Cognitive Psychology, 18(4), 452-99.

Samuel, A. G. (2001). Knowing a Word Affects the Fundamental Perception of The Sounds Within it. Psychological Science, 12(4), 348-351. doi:10.1111 / $1467-9280.00364$

Samuel, A. G. \& Kat, D. (1996). Early levels of analysis of speech. Journal of Experimental Psychology: Human Perception and Performance, 22(3), 676.

Samuel, A. G. \& Newport, E. L. (1979). Adaptation of speech by nonspeech: evidence for complex acoustic cue detectors. Journal of Experimental Psychology: Human Perception and Performance, 5(3), 563-78.

Sanborn, A. N., Griffiths, T. L., \& Navarro, D. J. (2010). Rational approximations to rational models: Alternative algorithms for category learning. Psychological Review, 117(4), 1144-67. doi:10.1037/a0020511

Sawusch, J. R. (1977). Peripheral and central processes in selective adaptation of place of articulation in stop consonants. The Journal of the Acoustical Society of America, 62(3), 738-50.

Schouten, M. (1980). The case against a speech mode of perception. Acta Psychologica, 44(1), 71-98. doi:10.1016/0001-6918(80)90077-3

Schreiber, E., Onishi, K., \& Clayards, M. A. (2013). Manipulating phonological boundaries using distributional cues. In Proceedings of meetings on acoustics (Vol. 19, 060298). Acoustical Society of America. doi:10.1121/1.4801082

Seydell, A., Knill, D. C., \& Trommershäuser, J. (2010). Adapting internal statistical models for interpreting visual cues to depth. Journal of vision, 10(4), 1.1-27. doi:10.1167/10.4.1 
Sharpee, T. O., Sugihara, H., Kurgansky, A. V., Rebrik, S. P., Stryker, M. P., \& Miller, K. D. (2006). Adaptive filtering enhances information transmission in visual cortex. Nature, 439(7079), 936-42. doi:10.1038/nature04519

Sheffert, S. M., Pisoni, D. B., Fellowes, J. M., \& Remez, R. E. (2002). Learning to recognize talkers from natural, sinewave, and reversed speech samples. Journal of Experimental Psychology: Human Perception and Performance, 28(6), 1447-1469. doi:10.1037/ /0096-1523.28.6.1447

Shi, L., Griffiths, T. L., Feldman, N. H., \& Sanborn, A. N. (2010). Exemplar models as a mechanism for performing Bayesian inference. Psychonomic Bulletin \& Review, 17(4), 443-64. doi:10.3758/PBR.17.4.443

Shinoda, K. \& Lee, C.-H. [C.-H.]. (2001). A structural Bayes approach to speaker adaptation. IEEE Transactions on Speech and Audio Processing, 9(3), 276-287. doi:10.1109/89.906001

Sidaras, S. K., Alexander, J. E. D., \& Nygaard, L. C. (2009). Perceptual learning of systematic variation in Spanish-accented speech. The Journal of the Acoustical Society of America, 125(5), 3306-16. doi:10.1121/1.3101452

Sidaras, S. K. \& Nygaard, L. C. (2014). Illusory Vocal Accommodation as a Function of Expected Age of the Speaker. Manuscript submitted for publication.

Simmons, J. P., Nelson, L. D., \& Simonsohn, U. (2011). False-Positive Psychology: Undisclosed Flexibility in Data Collection and Analysis Allows Presenting Anything as Significant. Psychological Science. doi:10.1177 / 0956797611417632

Simoncelli, E. P. \& Olshausen, B. A. (2001). Natural image statistics and neural representation. Annual Review of Neuroscience, 24(1), 1193-216. doi:10. 1146/annurev.neuro.24.1.1193

Sissons, H. T. \& Miller, R. R. (2009). Spontaneous recovery of excitation and inhibition. Journal of experimental psychology. Animal behavior processes, 35(3), 419-26. doi:10.1037/a0014815 
Smith, E. C. \& Lewicki, M. S. (2006). Efficient auditory coding. Nature, 439(7079), 978-82. doi:10.1038/nature04485

Smith, N. J. \& Levy, R. (2013). The effect of word predictability on reading time is logarithmic. Cognition, 128(3), 302-19. doi:10.1016/j.cognition.2013.02. 013

Smits, R. (2001). Hierarchical categorization of coarticulated phonemes: A theoretical analysis. Perception \& Psychophysics, 63(7), 1109-1139. doi:10.3758/ BF03194529

Sonderegger, M. \& Yu, A. (2010). A rational account of perceptual compensation for coarticulation. In S. Ohlsson \& R. Catrambone (Eds.), Proceedings of the 32nd annual conference of the cognitive science society (pp. 375-380). Austin, TX: Cognitive Science Society.

Stan Development Team. (2015). Stan: A C++ Library for Probability and Sampling, Version 2.9.0.

Staub, A. \& Clifton, C. (2006). Syntactic prediction in language comprehension: evidence from either...or. Journal of Experimental Psychology: Learning, Memory, and Cognition, 32(2), 425-36. doi:10.1037/0278-7393.32.2.425

Staum Casasanto, L. (2008). Does Social Information Influence Sentence Processing ? In B. C. Love, K. McRae, \& V. M. Sloutsky (Eds.), Proceedings of the 30th annual conference of the cognitive science society (pp. 799-804). Austin, TX: Cognitive Science Society.

Steinschneider, M., Schroeder, C. E., Arezzo, J. C., \& Vaughan, H. G. (1994). Speech-evoked activity in prima auditory cortex : effects of voice onset time. Science, 92, 30-43.

Stocker, A. A. \& Simoncelli, E. P. (2006). Sensory Adaptation within a Bayesian Framework for Perception. In Y. Weiss, B. Schölkoph, \& J. Platt (Eds.), Advances in neural information processing systems (Vol. 18, pp. 1291-1298). Cambridge, MA: MIT Press. 
Strand, E. A. (1999). Uncovering the Role of Gender Stereotypes in Speech Perception. Journal of Language and Social Psychology, 18(1), 86-100. doi:10. 1177/0261927X99018001006

Strand, E. A. \& Johnson, K. (1996). Gradient and Visual Speaker Normalization in the Perception of Fricatives. In D. Gibbons (Ed.), Natural language processing and speech technology: results of the 3rd konvens conference, bielfelt (pp. 14-26). Berlin: Mouton de Gruyter.

Strange, W. (1989). Evolving theories of vowel perception. The Journal of the Acoustical Society of America, 85(5), 2081. doi:10.1121/1.397860

Sumner, M. (2011). The role of variation in the perception of accented speech. Cognition, 119(1), 131-6. doi:10.1016/j.cognition.2010.10.018

Sumner, M. \& Samuel, A. G. (2009). The effect of experience on the perception and representation of dialect variants. Journal of Memory and Language, 60(4), 487-501. doi:10.1016/j.jml.2009.01.001

Tanenhaus, M. K., Spivey-Knowlton, M. J., Eberhard, K. M., \& Sedivy, J. C. (1995). Integration of visual and linguistic information in spoken language comprehension. Science, 268(5217), 1632-4.

Tenenbaum, J. B. \& Griffiths, T. L. (2001). Generalization, similarity, and Bayesian inference. Behavioral and Brain Sciences, 24(4), 629-40, 629-40.

Thanellou, A. \& Green, J. T. (2011). Spontaneous recovery but not reinstatement of the extinguished conditioned eyeblink response in the rat. Behavioral neuroscience, 125(4), 613-25. doi:10.1037/a0023582

Thomas, E. R. (2002). Sociophonetic Applications of Speech Perception Experiments. American Speech, 77(2), 115. doi:10.1215/00031283-77-2-115

Toscano, J. C. \& McMurray, B. (2010). Cue integration with categories: Weighting acoustic cues in speech using unsupervised learning and distributional statistics. Cognitive Science, 34(3), 434-464. doi:10.1111/ j. 1551 6709.2009.01077.x 
Toscano, J. C., Munson, C. M., Kleinschmidt, D. F., \& Jaeger, T. F. (n.d.). A single mechanism for language learning across the lifespan. Manuscript submitted for publication.

Trueswell, J. C. \& Tanenhaus, M. K. (1994). Semantic influences on parsing: Use of thematic role information in syntactic ambiguity resolution. Journal of Memory and Language, 33, 285-318.

Tunley, A. (1999). Coarticulatory influences of liquids on vowels in English Alison (Doctoral dissertation, University of Cambridge).

Tzeng, C. Y., Alexander, J. E. D., Sidaras, S. K., \& Nygaard, L. C. (2014). The Role of Training Structure in Perceptual Learning of Accented Speech. Manuscript submitted for publication.

Vallabha, G. K., McClelland, J. L., Pons, F., Werker, J. F., \& Amano, S. (2007). Unsupervised learning of vowel categories from infant-directed speech. Proceedings of the National Academy of Sciences of the United States of America, 104(33), 13273-8. doi:10.1073/pnas.0705369104

van den Bosch, A. \& Daelemans, W. (2013). Implicit Schemata and Categories in Memory-based Language Processing. Language and Speech, 56(3), 309328. doi:10.1177/0023830913484902

van der Zande, P., Jesse, A., \& Cutler, A. (2014). Cross-speaker generalisation in two phoneme-level perceptual adaptation processes. Journal of Phonetics, 43, 38-46. doi:10.1016/j.wocn.2014.01.003

van Wassenhove, V., Grant, K. W., \& Poeppel, D. (2005). Visual speech speeds up the neural processing of auditory speech. Proceedings of the National Academy of Sciences of the United States of America, 102, 1181-1186. doi:10. 1073/pnas.0408949102

Vatakis, A. \& Spence, C. (2007). Crossmodal binding: evaluating the "unity assumption" using audiovisual speech stimuli. Perception \& psychophysics, 69(5), 744-56. 
Vitevitch, M. S. \& Luce, P. A. (2004). A web-based interface to calculate phonotactic probability for words and nonwords in English. Behavior Research Methods, Instruments, \& Computers, 36(3), 481-7.

von Helmholtz, H. (1924). Treatise On Physiological Optics. Rochester, NY: The Optical Society of America.

Vroomen, J., van Linden, S., de Gelder, B., \& Bertelson, P. (2007). Visual recalibration and selective adaptation in auditory-visual speech perception: Contrasting build-up courses. Neuropsychologia, 45(3), 572-7. doi:10.1016/ j.neuropsychologia.2006.01.031

Vroomen, J., van Linden, S., Keetels, M., de Gelder, B., \& Bertelson, P. (2004). Selective adaptation and recalibration of auditory speech by lipread information: dissipation. Speech Communication, 44(1-4), 55-61. doi:10.1016/ j.specom.2004.03.009

Wainwright, M. J. (1999). Visual adaptation as optimal information transmission. Vision Research, 39(23), 3960-3974. doi:10.1016/S0042-6989(99)001017

Warker, J. A. \& Dell, G. S. (2006). Speech errors reflect newly learned phonotactic constraints. Journal of Experimental Psychology: Learning, Memory, and Cognition, 32(2), 387-98. doi:10.1037/0278-7393.32.2.387

Warker, J. A., Xu, Y., Dell, G. S., \& Fisher, C. (2009). Speech errors reflect the phonotactic constraints in recently spoken syllables, but not in recently heard syllables. Cognition, 112(1), 81-96. doi:10.1016/j.cognition.2009.03. 009

Weatherholtz, K. \& Jaeger, T. F. (2016). Speech perception and generalization across talkers and accents. In Oxford research encyclopedia of linguistics.

Weatherholtz, K., Seifeldin, M., Kleinschmidt, D. F., Kurumada, C., \& Jaeger, T. F. (2016). Speech perception as probabilistic inference under uncertainty based on social-indexical knowledge. Manuscript submitted for publication. 
Webster, M. A., Werner, J. S., \& Field, D. J. (2005). Adaptation and the Phenomenology of Perception. In C. Clifford \& G. Rhodes (Eds.), Fitting the mind to the world: adaptation and after-effects in high-level vision (advances in visual cognition) (Vol. 2, pp. 241-277). Oxford University Press.

Wei, X. \& Stocker, A. A. (2012). Efficient coding provides a direct link between prior and likelihood in perceptual Bayesian inference. Advances in Neural Information Processing Systems, 25(May), 1-9.

Wells, J. B., Christiansen, M. H., Race, D. S., Acheson, D. J., \& MacDonald, M. C. (2009). Experience and sentence processing: statistical learning and relative clause comprehension. Cognitive Psychology, 58(2), 250-71. doi:10. 1016/j.cogpsych.2008.08.002

Whalen, D. H. (1984). Subcategorical phonetic mismatches slow phonetic judgments. Perception \& psychophysics, 35(1), 49-64.

White, K. S. \& Aslin, R. N. (2011). Adaptation to novel accents by toddlers. Developmental Science, 14(2), 372-384. doi:10.1111/j.1467-7687.2010.00986. $\mathrm{x}$

Wonnacott, E., Newport, E. L., \& Tanenhaus, M. K. (2008). Acquiring and processing verb argument structure: distributional learning in a miniature language. Cognitive psychology, 56(3), 165-209. doi:10.1016/j.cogpsych. 2007.04.002

Yildirim, I., Degen, J., Tanenhaus, M. K., \& Jaeger, T. F. (2013). Linguistic Variability and Adaptation in Quantifier Meanings. In M. Knauff, M. Pauen, N. Sebanz, \& I. Wachsmuth (Eds.), Proceedings of the 35th annual conference of the cognitive science society (pp. 3835-40). Austin, TX: Cognitive Science Society.

Yildirim, I., Degen, J., Tanenhaus, M. K., \& Jaeger, T. F. (2016). Talker-specificity and adaptation in quantifier interpretation. Journal of Memory and Language, 87, 128-143. doi:10.1016/j.jml.2015.08.003 
Zaki, S. R. \& Kleinschmidt, D. F. (2014). Procedural memory effects in categorization: Evidence for multiple systems or task complexity? Memory $\mathcal{E}$ cognition, 42(3), 508-24. doi:10.3758/s13421-013-0375-9

Zäske, R., Fritz, C., \& Schweinberger, S. R. (2013). Spatial inattention abolishes voice adaptation. Attention, perception \& psychophysics, 75(3), 603-13. doi:10.3758/s13414-012-0420-y

Zhu, X., Rogers, T., Qian, R., \& Kalish, C. (2007). Humans Perform SemiSupervised Classification Too. In A. Cohn (Ed.), Proceedings of the 22nd aaai conference on artificial intelligence (pp. 864-869). Menlo Park, CA: AAAI Press. 


\section{A Ideal adapter modeling methods and assumptions}

This appendix lays out the basic Bayesian belief updating model proposed as part of the ideal adapter framework. We first summarize the formal specification of the model. As we describe in the main text, the model assumes that adaptation takes place over a cue dimension that might integrate auditory and visual information. The arguments for this assumption, potential caveats, and our reply to those caveats are summarized next. We then describe how the model's parameters were fit to the behavioral data from Vroomen, van Linden, de Gelder, and Bertelson (2007) and our own study. Throughout these sections we state the assumptions made by our model and model fitting procedure. Crucially, none of these assumptions creates a bias in favor of our hypothesis. Finally, we provide a table that summarizes our assumptions.

\section{A.1 Model specification}

To quantify and test the qualitative predictions of the ideal adapter framework, we implemented a basic Bayesian belief updating model. This model makes a number of simplifying assumptions. The most notable is that we consider only two categories in this model, /b/ and /d/, and assume that the prior beliefs 
about their means and variances are independent

$$
p\left(\mu, \sigma^{2}\right)=\prod_{c} p\left(\mu_{c}, \sigma_{c}^{2}\right)=p\left(\mu_{\mathrm{b}}, \sigma_{\mathrm{b}}^{2}, \mu_{\mathrm{d}}, \sigma_{\mathrm{d}}^{2}\right)
$$

Combined with the fact that participants in these experiments reliably classify even the auditorily ambiguous audio-visual adaptors as the intended categories (Vroomen, van Linden, Keetels, et al., 2004), this simplifies belief updating (Equation 2.8).

The form of the individual category parameter priors was also chosen for reasons of computational convenience. We use the conjugate prior for the Normal distribution with unknown mean and variance, a Normal- $\chi^{-2}$ distribution (Gelman, Carlin, et al., 2003). This distribution factorizes the joint prior into two components:

$$
\begin{aligned}
p\left(\mu_{c}, \sigma_{c}^{2}\right) & =p\left(\mu_{c} \mid \sigma_{c}^{2}\right) p\left(\sigma_{c}^{2}\right) \\
& =\operatorname{Normal}\left(\mu_{c} \mid \mu_{0, c}, \sigma_{c}^{2} / \kappa_{0}\right) \chi^{-2}\left(\sigma_{c}^{2} \mid v_{0}, \sigma_{c, 0}^{2}\right)
\end{aligned}
$$

The prior on the variance is a $\chi^{-2}$ distribution, with two parameters, $v_{0}$ and $\sigma_{c, 0}^{2} . \sigma_{c, 0}^{2}$ is the expected value of the category variance $\sigma_{c}^{2}$, while $v_{0}$ represents the effective prior sample size for the mean (reflecting the uncertainty about $\sigma_{c, 0}^{2}$, see main text)The prior on the mean is conditioned on the value of the category variance, and is a Normal distribution. The expected value of that normal distribution is the prior mean parameter $\mu_{0, c}$. Its variance is $\sigma_{c}^{2} / \kappa_{0}$, i.e., the category variance divided by the effective prior sample size for the mean $\kappa_{0}$.

\section{A.1.1 Belief updating with a conjugate prior}

Using a conjugate prior is convenient because after updating with observations $X=x_{1}, \ldots, x_{n}$ (whose mean value is $\bar{x}$ and sample variance is $s^{2}=\frac{1}{n} \sum_{i}\left(x_{i}-\right.$ 
$\left.\bar{x})^{2}\right)$, the posterior is also a Normal- $\chi^{-2}$ distribution, with updated parameters (Gelman, Carlin, et al., 2003):

$$
\begin{aligned}
\kappa_{n} & =\kappa_{0}+n \\
\mu_{n, c} & =\frac{\kappa_{0}}{\kappa_{n}} \mu_{0, c}+\frac{n}{\kappa_{n}} \bar{x} \\
v_{n} & =v_{0}+n \\
\sigma_{n, c}^{2} & =\frac{1}{v_{n}}\left(v_{0} \sigma_{c, 0}^{2}+n s^{2}+\frac{n \kappa_{0}}{\kappa_{n}}\left(\mu_{0, c}-\bar{x}\right)^{2}\right)
\end{aligned}
$$

These parameter updates have intuitive interpretations: the $\kappa_{0}$ and $v_{0}$ parameters are 'pseudocounts', or the effective sample size of the prior. To update them from their prior to posterior values, they are both incremented by $n$, the number of observations. The updated mean $\mu_{n, c}$ is a weighted average of the sample mean (weighted by the actual sample size $n$ ) and the prior mean (weighted by the prior effective sample size of the mean $\kappa_{0}$ ). The updated expected variance $\sigma_{n, c}^{2}$ is also a weighted average, of the observed variance $n s^{2}$ (weighted by the actual sample size $n$ ), the prior expected variance $\sigma_{0, c}^{2}$ (weighted by the effective prior sample size, $v_{0}$ ), and a third term, which accounts for deviation of the observed mean from the expected mean $\mu_{c, 0}$. This last term is weighted by $n \kappa_{0} / \kappa_{n}$, which gets larger (relative to the weights of the other terms, $n$ and $v_{0}$ ) when $n \approx \kappa_{0}$.

What happens to the expected mean and variance when more and more observations are made? Specifically, what happens when $n$ becomes much larger than the prior effective sample sizes for the variance $v_{0}$ and mean $\kappa_{0}$ ? First, the posterior mean $\mu_{n}$ converges to the observed mean $\bar{x}$ (since $n / \kappa_{n}=$ $n /\left(\kappa_{0}+n\right) \approx 1$ when $\left.n \gg \kappa_{0}\right)$. Second, and similarly, the expected variance converges on the observed variance. That is, with a lot of data about the current situation the model's beliefs will converge against the actual statistics of that situation. In both cases, the stronger the prior beliefs (larger prior sample 
sizes $\kappa_{0}$ and $v_{0}$ ), the more observations it takes to overcome the listener's prior beliefs. These prior effective sample size parameters can thus be understood as controlling the strength and speed of adaptation effects.

\section{A.1.2 Incremental belief updating}

If all the individual, observed cue values for each category are assumed to be statistically independent (conditioned on the mean and variance), then the joint likelihood of all observed cues is equal to the product of the individual likelihoods, and thus

$$
\begin{aligned}
p\left(\mu_{c}, \sigma_{c}^{2} \mid x_{1} \ldots x_{N}\right) & \propto \underbrace{p\left(x_{1}, \ldots, x_{N} \mid \mu_{c}, \sigma_{c}^{2}\right)}_{\text {joint likelihood }} \underbrace{p\left(\mu_{c}, \sigma_{c}^{2}\right)}_{\text {prior }} \\
& \propto\left(\prod_{i=1}^{N} p\left(x_{i} \mid \mu_{c}, \sigma_{c}^{2}\right)\right) p\left(\mu_{c}, \sigma_{c}^{2}\right) \\
& \propto p\left(x_{N} \mid \mu_{c}, \sigma_{c}^{2}\right)\left(\prod_{i=1}^{N-1} p\left(x_{i} \mid \mu_{c}, \sigma_{c}^{2}\right)\right) p\left(\mu_{c}, \sigma_{c}^{2}\right) \\
& \propto \underbrace{p\left(x_{N} \mid \mu_{c}, \sigma_{c}^{2}\right)}_{\text {likelihood of } x_{N}} \underbrace{p\left(\mu_{c}, \sigma_{c}^{2} \mid x_{1}, \ldots, x_{N-1}\right)}_{\text {updated prior }}
\end{aligned}
$$

That is, the listener's beliefs after the Nth observation are a combination of their beliefs about the means and variances after all $N-1$ preceding observations, combined with the likelihood of the current observation given those beliefs.

One insight this provides is that it is not always necessary to maintain a full record of all observations made so far, or even their statistics. Rather, it is sufficient to just track the posterior distribution over means and variances after each token. This leads naturally to approximate inference methods like particle filters, which approximate beliefs after $N-1$ observations via a set of "particles", each a particular set of means and variances, with the particles collectively approximating the full distribution $p\left(\mu_{c}, \sigma_{c}^{2}\right)$. After observation $N$, each particle's 
estimate is updated, with particles failing to predict observation $N$ effectively being thrown out and particles which do effectively predict observation $N$ persisting or even being "cloned" to replace the rejected particles. Particle filters have been shown to be a reasonable approximation of Bayes-optimal inference in distributional learning categorization problems, and match human performance well even with a small number of particles (Sanborn et al., 2010).

\section{A.2 Audio-visual cue integration}

Next, we discuss the model's assumptions about the nature of the dimension over which adaptation takes place. This part of the model is not a consequences of the ideal adapter framework. Instead, it is motivated by evidence for crossmodal interactions in audio-visual speech processing (Bejjanki, Clayards, et al., 2011; McGurk \& J. MacDonald, 1976). Given evidence for such cross-modal interactions, we remain agnostic about the level at which listeners are adapting to the audio-visual stimuli. Specifically, we treat as a free parameter whether listeners adapt to a purely auditory representation of the perceived cues, or to some representation which integrates information from both auditory and visual cues. After describing how this was implemented in the model, we summarize possible objections to this aspect of the model and our reply to these objections.

\section{A.2.1 Linear cue combination}

Under reasonably general assumptions, information from auditory and visual cues to the same phonetic dimension can be optimally combined into a multimodal cue value by a weighted sum $x=w_{a} x^{(a)}+w_{v} x^{(v)}$, where the weights $w_{a}$ and $w_{v}$ sum to 1 and are proportional to the reliability of the auditory and 
visual cues (Bejjanki, Clayards, et al., 2011; Ernst \& Banks, 2002; Jacobs, 2002; Knill \& Saunders, 2003; Toscano \& McMurray, 2010).

We incorporate this into our model via treating the perceived cue values $x$ as a weighted sum of the continuum values for the auditory and visual tokens $x=w x^{(v)}+(1-w) x^{(a)}$, where the weight $w$ is a free parameter. For the audiovisual adaptors used in this experiment, in the /b/ condition the visual cue indicates a prototypical / b/, and so $x^{(v)}=1$, while the auditory cue indicates an ambiguous $/ \mathrm{b} /-/ \mathrm{d} /, x^{(a)}=x_{\mathrm{bd}} \approx 5$ (depending on the particular participant's most ambiguous stimulus).

The linear combination of these two cues results in an integrated cue estimate somewhere in between, not quite prototypical but not fully ambiguous. Note that for our model fits, the best-fitting cue weights are in general roughly equal $(w \approx 0.5)$, which suggests that the perceived cue value for the audiovisual adaptor is substantially less ambiguous than the auditory cue. This is hardly surprising given well-known cross-modal effects on speech perception with similar consequences, such as the McGurk effect (McGurk \& J. MacDonald, 1976). The assumption that the audio-visual stimulus is not really ambiguous is also consistent with our finding from pilot studies that participants can reliably classify the ambiguous audiovisual adaptor stimuli (which also justifies somewhat our assumption-made solely for the sake of convenience- that the category label of each adaptor stimulus is known with certainty).

\section{A.2.2 Is there evidence against audio-visual integration in adaptation?}

Our decision to include audio-visual cue integration in the model is supported by a wealth of evidence that audio and visual cues are processed together in speech perception (e.g., Bejjanki, Clayards, et al., 2011; Massaro, 2004; van 
Wassenhove, Grant, \& Poeppel, 2005; Vatakis \& Spence, 2007). There are, however, two studies that have looked specifically at selective adaptation to audio-visual adaptors, and contrary to what we propose here, have concluded that selective adaptation is driven entirely by the adaptor's audio component (Roberts \& Summerfield, 1981; Saldaña \& Rosenblum, 1994). Since these studies might be taken to argue that we introduce unnecessary complexity into the model, we briefly discuss them.

The audio-visual adaptors used by Roberts and Summerfield (1981) and Saldaña and Rosenblum (1994) differ from the adaptors used by Vroomen, van Linden, de Gelder, and Bertelson (2007) and our replication, in that the audio and visual components had large, categorical mismatches. Roberts and Summerfield (1981) used auditory-/b/, visual-/g/ adaptors, intended to evoke a /d/ percept (as in McGurk \& J. MacDonald, 1976), but found selective adaptation effects on $\mathrm{a} / \mathrm{b} /-/ \mathrm{d} /$ continuum that were indistinguishable from an audiovisual /b/ adaptor. However, their participants did not generally perceive the adaptor in this way, with only half reporting an alveolar $/ \mathrm{d} /$ or $/ ð /$, and the others reporting $/ \mathrm{kl} /, / \mathrm{m} /$, of $/ \mathrm{fl} /$. This contrasts with with the perception of the stimuli in Vroomen, van Linden, de Gelder, and Bertelson (2007) and our own studies, where participants reliably classified the audio-visual adaptor stimuli as 'labeled' by the visual component.

Saldaña and Rosenblum (1994) is more relevant for the current purpose. They used an audio-/ba/, visual-/va/ adaptor stimulus which was consistently identified as /va/ by participants. This produced a selective adaptation effect on a / ba/-/va/ continuum equivalent to that of its audio / ba/ component presented separately. However, it is not possible to tell whether the observed effect was due to selective adaptation of $/ \mathrm{b} /$, or recalibration of $/ \mathrm{v} /$, since both would produce a shift in the category boundary towards $/ \mathrm{b} /$. If the visual and auditory cues are integrated as we have tentatively proposed, and the visual cue 
weight is higher than the auditory weight, then the audio-visual integration would be an imperfect /v/, leading in our model to both a /v/ percept and recalibration of $/ \mathrm{v} /$ and fewer $/ \mathrm{b} /$ responses.

In sum, it is broadly accepted that speech perception involves cross-modal cue integration. Whether adaptation can take place over those integrated cues is an open question that previous literature does not speak to. Our own results suggest a positive answer (since the best-fitting visual cue weight $w \approx .5$ in all our studies).

\section{A.2.3 The (ir)relevance of audio-visual integration for the in- terpretation of our results}

Treating the adaptor cue value as a linear combination of the audio and visual cues has two main effects in our model. First, it causes recalibration to saturate somewhere below the maximum possible aftereffect of +1 (all adaptorcategory responses). Second, it causes recalibration to peak and then decrease with further exposure. Neither of these consequences comes from the combination of audio and visual cues in the model per se, but rather from the fact that such combination causes the adaptor percept to be perceived as not fully ambiguous.

There are other possible reasons this might occur besides cue integration, such as a perceptual magnet effect. Feldman, Griffiths, and Morgan (2009) explain the perceptual magnet effect as a result of the listener's attempt to infer what cue value the talker intended to produce based on an observed cue that is corrupted by noise variability or sensory uncertainty. ${ }^{1}$ In such a model, the lis-

\footnotetext{
${ }^{1}$ Here we use "noise" as a shorthand for noise and sensory uncertainty. Such uncertainty is not necessarily due to noise in the sense of random variability but also arises from, for instance, the limited resolution in the neural representation of particular stimulus parameters or a mismatch in the type of features encountered and those assumed by upstream neural decoding (Beck, Ma, Pitkow, Latham, \& Pouget, 2012).
} 
tener's knowledge of the category distributions acts as an additional cue, which is combined with the noisy percept. Many of the aspects of the perceptual magnet effect can be explained by assuming that what the listener perceives-for the purposes of making responses in a perceptual magnet experiment-is the best guess about the talker's intended production, which is a combination of the actual cue value perceived and the cue values expected based on the category structure. Furthermore, when the listener knows that the talker intended to produce a particular category, this framework predicts that the perceived cue is pulled towards that category mean, which is very similar to the effect of cue integration with a prototypical visual cue value.

Regardless of whether the perceptual magnet effect (through visual labeling of the auditory stimulus) or early audio-visual integration cause the audiovisual stimulus to be substantially less ambiguous than the auditory, the underlying (statistical) logic of the two approaches is very similar. The standard cue integration model (Ernst \& Banks, 2002) assumes that the perceiver is trying to get a good estimate of an (unobserved) quantity (like the talker's intended production) which is noisily approximated by multiple cues. When the two cues are corrupted by independent Gaussian noise, this gives rise to an optimal strategy of taking an average of the individual estimates yielded by the two cues, weighted according to their reliability. Similarly, in the perceptual magnet model of Feldman, Griffiths, and Morgan (2009), when the category and noise distributions are Gaussian, the best estimate of the intended cue value is the same, reliability- (inverse variance-) weighted average of the category mean and the observed cue value. ${ }^{2}$

\footnotetext{
${ }^{2}$ For the purposes of adaptation, the major practical difference between an explanation in terms of the perceptual magnet effect and an explanation in terms of cross-modal cue integration would be that as the listener updates their beliefs about the category mean, the value of the perceptual magnet cue would change with exposure, whereas the value of the visual cue (presumably) does not. Of relevance is evidence that prolonged repeated exposure can also erase lexically-driven recalibration (where there is no visual cue available): Vroomen, van Linden, de Gelder, and Bertelson (2007) re-analyzed data from Samuel (2001), and found that a
} 
Why would the ideal adapter combine information about the intended production from multiple cues before adaptation? Within-category variability is not only meaningless noise, but rather might additionally reflect factors like coarticulation, or other systematic changes in cue value. This means that the cue values we have treated as relevant only for making a single, categorical decision (e.g. /b/vs. /d/) are actually potentially informative about other nearby segments as well. Thus, the talker's intended cue value, in addition to their intended category, reflects nearby categories as well. This fact, combined with non-uniform transitional probabilities between different categories, means that when considered as a distribution over multiple cues, the distribution for each category can be thought of as highly structured, to the extent that meaningful variability in a cluster of cues is stronger than meaningless noise variability in those cues.

With multiple cues, within-category variability due to the influence of neighboring segments might thus lie a low-dimensional manifold, the shape and structure of which is determined in part by how possible contexts influence the cue values corresponding to each category. Treating the bottom-up sensory signal as a likelihood over intended productions (as do Feldman, Griffiths, \& Morgan, 2009) and combining it with a structured prior in essence filters the uncertain sensory estimates in such a way as to maximize information about meaningful within-category variation in cue values and minimize variability due to uninformative noise.

Using such an integrated cue value for belief updating would not be strictly optimal in a Bayesian sense. It could still, however, reflect constraints imposed by a system which is optimized for processing running speech, rather than es-

lexically disambiguated, auditorily ambiguous adaptor elicited the same pattern of initial positive and long-run negative aftereffects as their visually-disambiguated adaptor. This suggests that an explanation of long-run recalibration behavior which relies on adaptation to integration of audio-visual cues per se is inadequate. 
timating the distributions of raw acoustic cue values for each single category in isolation. Support for this idea comes from findings that listeners do use information about the onset of an upcoming noun contained in the vowel of the determiner 'the' to launch saccades to the corresponding target in a visual world task before the onset of the target noun itself (Salverda et al., 2014).

Next, we describe how the model was fit to the behavioral data from Vroomen, van Linden, de Gelder, and Bertelson (2007) and our own study.

\section{A.3 Model fitting and parameter estimation}

The updating rules of the conjugate prior (A.4) suggest natural ways of fitting the model to the data from Vroomen, van Linden, de Gelder, and Bertelson (2007). First, there is a natural separation between the expected value parameters, $\mu_{c, 0}$ and $\sigma_{c, 0}^{2}$, which determine the category means and variances the listener believes are most probable before the adaptation phase begins, and the effective prior sample size parameters, $\kappa_{0}$ and $v_{0}$, which determine how willing they are to update those beliefs. The expected means and variances can be set a priori, based on pre-test data. These are thus fixed by the data, rather than being free parameters. Only the effective prior sample size parameters must be fit to the actual adaptation data.

While it is in principle possible to fit each participant's data individually, the amount of data available from each participant is very small (only six trials per test block) and leads to unstable parameter estimates. We thus chose to use the aggregate data. Another possibility would be to fit a model with a linked prior on the hyperparameters $v_{0}, \kappa_{0}$, and $w$ that allows for systematically limited variability between listeners. While the insights into possible individual differences would be enlightening, the primary purpose of the current study is 
to demonstrate the mechanics of the proposed framework. We therefore leave additional modeling improvements to future work.

\section{A.3.1 Estimating prior expected means and variances from pre- test data}

We used the pre-test classification data collected by Vroomen, van Linden, de Gelder, and Bertelson (2007) to estimate the underlying means and variances of the corresponding Gaussian mixture (Feldman, Griffiths, \& Morgan, 2009).

For a mixture of two Gaussian distributions-/b/and /d/-with equal variance $\sigma^{2}$, the categorization function $p(C=\mathrm{b} \mid x)$ is a logistic function $(1+$ $\exp (-g x+b))^{-1}$, with slope $g$ and intercept $b$ related to the means $\mu_{\mathrm{b}}$ and $\mu_{\mathrm{d}}$ and the variance $\sigma^{2}$ :

$$
g=\frac{\mu_{\mathrm{b}}-\mu_{\mathrm{d}}}{\sigma^{2}} \quad \text { and } \quad b=\frac{\mu_{\mathrm{b}}^{2}-\mu_{\mathrm{d}}^{2}}{\sigma^{2}}=\frac{\left(\mu_{\mathrm{b}}+\mu_{\mathrm{d}}\right)\left(\mu_{\mathrm{b}}-\mu_{\mathrm{d}}\right)}{\sigma^{2}}
$$

To estimate $b$ and $g$ from the pre-test data, one additional degree of freedom in Equation A.12 needs to be held constant. We chose to fix the distance between the means, $\mu_{\mathrm{b}}-\mu_{\mathrm{d}}$. Given these values, the values for $\left(\mu_{\mathrm{b}}+\mu_{\mathrm{d}}\right) / 2$ (the middle of the participant's subjective continuum) and $\sigma^{2}$ can be calculated using

$$
\frac{\mu_{\mathrm{b}}+\mu_{\mathrm{d}}}{2}=\frac{b}{g} \quad \text { and } \quad \sigma^{2}=\frac{\mu_{\mathrm{b}}-\mu_{\mathrm{d}}}{g}
$$

The difference between the means sets the scale of the continuum, and we chose to use $\mu_{\mathrm{b}}-\mu_{\mathrm{d}}=8$, the length (in steps) of the acoustic continuum, which stretches from $x=1$ (derived from a natural /aba/) to $x=9$ (from a natural /ada/). This is roughly equivalent to assuming that all subjects would accept these tokens as good productions of /aba/ and /ada/, which indeed they do (Vroomen, van Linden, Keetels, et al., 2004). 
This method makes two assumptions. First, it assumes that participant's subjective prior probabilities of /b/ vs. /d/ (regardless of the cue value) are equal. This is not difficult to relax (it only shifts the boundary of the classification function by the log ratio of the prior probabilities; Feldman, Griffiths, \& Morgan, 2009), and the model's predictions are qualitatively unchanged when the prior probability of /b/vs. /d/ is included as a free parameter.

Second, it assumes that the prior variance of the two categories is equal. There are two reasons why this assumption (though probably false) is sufficient for our purpose. First, based on pilot simulations, asymmetric prior variance results in asymmetries between recalibration by $x_{\mathrm{bd}}^{\mathrm{b}}$ and $x_{\mathrm{bd}}^{\mathrm{d}}$ which appear as a overall bias towards more $/ \mathrm{b} /$ or $/ \mathrm{d} /$ responses with further exposure, regardless of the exposure category. Using the aftereffect difference score as the dependent measure largely removes any effect of this bias, because taking the difference between the $/ \mathrm{b} /$ and $/ \mathrm{d} /$ exposure conditions removes this positive correlation (see Figure 2.5). Second, and more importantly, asymmetric prior variance does not change the qualitative predictions about the build-up and decay of recalibration overall, and the purpose of setting prior parameters based on non-adaptation data is to reduce the flexibility of the model in order to more clearly evaluate the hypothesis that phonetic adaptation reflects incremental belief updating. ${ }^{3}$

Individual listeners' classification functions show a fair amount of variability, but all have comparable slopes and boundaries roughly in the middle of the continuum. For this reason, we fit a mixed effects logistic regression model (Jaeger, 2008) to the pre-test data, which allows for some variability in the

\footnotetext{
${ }^{3}$ Note that it would in theory be possible to estimate prior category variances directly from other data, removing the need for the simplifying assumption of equal prior variances. For example, prior variances could be estimated from aggregate or even individual production data, discriminability data (Kronrod et al., 2012), goodness-of-exemplar judgments (Pisoni \& Tash, 1974; Andruski, Blumstein, \& Burton, 1994) or any combination thereof. The exploration of these directions for model improvement are left for future work.
} 
slope and intercept of each subject, and so better estimates the slope and intercept most representative of the population. The prior parameters were set based on this slope and intercept as above, with $\mu_{\mathrm{b}, 0}=1.10, \mu_{\mathrm{d}, 0}=9.10$ and $\sigma_{\mathrm{b}, 0}^{2}=\sigma_{\mathrm{d}, 0}^{2}=3.74$.

\section{A.3.2 Generating predictions from prior confidence and visual cue weight hyperparameters}

The free parameters $-v_{0}, \kappa_{0}$, and $w-$ were fit to the listeners' responses during test trials, which occurred after exposure to $1,2,4, \ldots, 256$ adaptor stimuli.

Model predictions were generated for each test block in the following way. For a test block after $n$ cumulative exposures to the /b/ adaptor, model predictions are generated assuming that the observed values are $n$ repetitions of the most ambiguous cue value $\left(X=\left\{x_{1}, \ldots, x_{n}\right\}, x_{i}=x_{\mathrm{bd}}\right)$ which are labeled as /b/ with very high certainty $\left(C=\left\{c_{1}, \ldots, c_{n}\right\}, c_{i}=b\right)$ by the visual component (and vice-versa for /d/ exposure trials). The response of the ideal adapter to test stimulus $x_{\text {test }}$ depends on the posterior distribution over category parameters given the exposure to the adaptor thus far, $p\left(\mu_{\mathrm{b}}, \sigma_{\mathrm{b}}^{2} \mid X, C\right)$ :

$$
\begin{aligned}
p\left(c_{\text {test }}=\mathrm{b} \mid x_{\text {test }}, X, C\right) & =\iint p\left(c_{\text {test }}=\mathrm{b}, \mu_{\mathrm{b}}, \sigma_{\mathrm{b}}^{2} \mid x_{\text {test }}, X, C\right) \mathrm{d} \mu_{\mathrm{b}} \mathrm{d} \sigma_{\mathrm{b}}^{2} \\
& =\iint p\left(c_{\text {test }}=\mathrm{b} \mid x_{\text {test }}, \mu_{\mathrm{b}}, \sigma_{\mathrm{b}}^{2}\right) p\left(\mu_{\mathrm{b}}, \sigma_{\mathrm{b}}^{2} \mid X, C\right) \mathrm{d} \mu_{\mathrm{b}} \mathrm{d} \sigma_{\mathrm{b}}^{2}
\end{aligned}
$$

Because of the conjugate prior we used, the posterior $p\left(\mu_{\mathrm{b}}, \sigma_{\mathrm{b}}^{2} \mid X, C\right)$ is found analytically, by updating the hyperparameters $\left(\kappa_{0}, \nu_{0}, \mu_{\mathrm{b}, 0}, \sigma_{\mathrm{b}, 0}^{2}\right)$ as described above in equations (A.4)-(A.7). Specifically, they were updated with the sample statistics, which have count $n$, mean $\bar{x}=w x_{\text {visual }}+(1-w) x_{\mathrm{bd}}$ (where the visual cue value $x_{\text {visual }}=1$ for visual $/ \mathrm{b} /$ and $x_{\text {visual }}=9$ for visual 
$/ \mathrm{d} /)$, and sample variance $s^{2}=0$.

The particular choice of prior is also convenient in that the integral in (A.14) can be evaluated analytically, with the result that the marginal likelihood $p\left(x_{\text {test }} \mid c_{\text {test }}=\mathrm{b}, X, C\right)$ has a scaled $t$ distribution, with mean $\mu_{\mathrm{b}, n}$, variance (squared scale) $\frac{\left(1+\kappa_{n}\right) \sigma_{\mathrm{b}, n}^{2}}{\kappa_{n}}$, and degrees of freedom $v_{n}$ (Gelman, Carlin, et al., 2003). The marginal likelihood of the $x_{\text {test }}$ under the other, un-adapted category, is analogously found from the prior hyperparameters, $\kappa_{0}, \nu_{0}, \mu_{\mathrm{d}, 0}, \sigma_{\mathrm{d}, 0}^{2}$ and the marginal posterior probability $p\left(c_{\text {test }}=\mathrm{b} \mid x_{\text {test }}, X, C\right)$ can then be found for each test stimulus based on Equation A.15. The marginal posterior probabilities for the three types of test stimuli are averaged to produce the model-predicted probability of a /b/ or /d/ response on test block $n$ (depending on whether the visual cue indicated /b/ or /d/, respectively).

\section{A.3.3 Likelihood of test stimuli}

To fit to the overall data, the likelihood of the data was calculated based on the number of responses that were the same category as the adaptor. These adaptor-category response counts were summed in each block. That is, for a /b/-exposure block, /b/ responses were considered 'positive' responses, while /d/ responses were considered 'positive' responses for test blocks during /d/ exposure. This adaptor-category response rate is essentially equivalent to the aftereffect difference score: if $y_{b}$ and $y_{d}$ denote the proportion of $/ \mathrm{b} /$ responses after $/ \mathrm{b} /$ and $/ \mathrm{d} /$ exposure respectively, $z_{b}=y_{b}$ and $z_{d}=1-y_{d}$ denote the proportion of adaptor category responses after $/ \mathrm{b} /$ and $/ \mathrm{d} /$ exposure, and $z=$ $\frac{z_{b}+z_{d}}{2}$ is the average adaptor category response overall, then the aftereffect can be found via $2 z-1=z_{b}+z_{d}-1=z_{b}-\left(1-z_{d}\right)=y_{b}-y_{d}=y_{\mathrm{AE}}$.

The likelihood of the adaptor-category response counts given the total number of trials and the model predicted adaptor-category response probability for 
each block and condition (derived from particular values of the hyperparameters $v_{0}, \kappa_{0}$, and $w$ ) was evaluated by a binomial likelihood distribution. Specifically, if $z_{j}$ is the number of adaptor-category responses at test block $j$, out of $n_{j}$ test trials in that block (in both cases summing across participants), and $\theta_{j}$ is the model-predicted probability of adaptor-category response, then the likelihood of block $j$ is

$$
p\left(y_{j} \mid n_{j}, \theta_{j}\right)=\left(\begin{array}{l}
n_{j} \\
y_{j}
\end{array}\right) \theta_{j}^{y_{j}}\left(1-\theta_{j}\right)^{n_{j}-y_{j}}
$$

and the joint likelihood of the data is

$$
p(y \mid n, \theta)=\prod_{j} p\left(y_{j} \mid n_{j}, \theta_{j}\right)
$$

Because a binomial likelihood was used for fitting the model to the data, the error bars on the data show the confidence intervals for the rate parameter of a binomial distribution with the observed counts of adaptor-category responses and non-adaptor-category responses. These were calculated as the $2.5 \%$ and $97.5 \%$ quantiles of the posterior distribution for the adaptor-category response rate, which is $\operatorname{Beta}\left(z_{j}+\frac{1}{2}, n_{j}-z_{j}+\frac{1}{2}\right)$ assuming a non-informative (Jeffrey's) Beta $\left(\frac{1}{2}, \frac{1}{2}\right)$ prior (Gelman, Carlin, et al., 2003). These quantiles were transformed to the aftereffect scale for visualization in the same way as the data, and for the proportion-/b/ response plots were calculated based on the number of $/ \mathrm{b} /$ and $/ \mathrm{d} /$ responses instead of the number of adaptor-category responses.

\section{A.3.4 Sampling and hyperpriors}

This joint data likelihood was combined with a weak, regularizing prior,

$$
p\left(\log \kappa_{0}\right)=p\left(\log v_{0}\right)=\operatorname{Normal}(0,100)
$$


which has a $95 \%$ interval that stretches from about $v_{0}=10^{-9}$ to $10^{9}$, with a mode of 1 . Any prior sample size that is a few times larger than the maximum sample size (256) results in essentially no adaptation; in this range the prior is essentially constant (the prior probability of $v_{0}=1$, the value which maximizes the prior probability, is only 1.2 times greater than the prior probability of $v_{0}=$ 1000 , and likewise for $\kappa_{0}$ ), and thus this prior has essentially no influence on the fit of the model. For the visual cue weight $w$ the prior was uniform between 0 and 1 and thus uninformative.

The posterior distribution of the hyperparameters is not easy to find analytically, and so samples were drawn from this distribution using a hybrid Gibbs/slice sampler, where each hyperparameter is sampled in turn via slice sampling (Neal, 2003), given the last sampled values of the other parameters. The samples can be used to find the maximum a posteriori (MAP) estimate of the best-fitting parameter values, as well as the full joint posterior. The joint posteriors of the confidence parameters (mean prior pseudocount $\kappa_{0}$ and variance prior pseudocount $v_{0}$ ) for the fits to the build-up of recalibration, build-up of selective adaptation, long-term effects in both, and the Mechanical Turk replication data are shown in Figure A.1.

\section{A.4 Model assumptions}

In order to relate the qualitative predictions of the ideal adapter framework to the behavior of human listeners, it is necessary or convenient to make some simplifying assumptions. Table A.1 review these assumptions, whether they are justified, and if not, whether violating them leads to problems for the conclusions we reach from our modeling results. Many of them have already been introduced and discussed above. None of these assumptions bias the results of the modeling towards better fits. If anything many of them make it harder for 


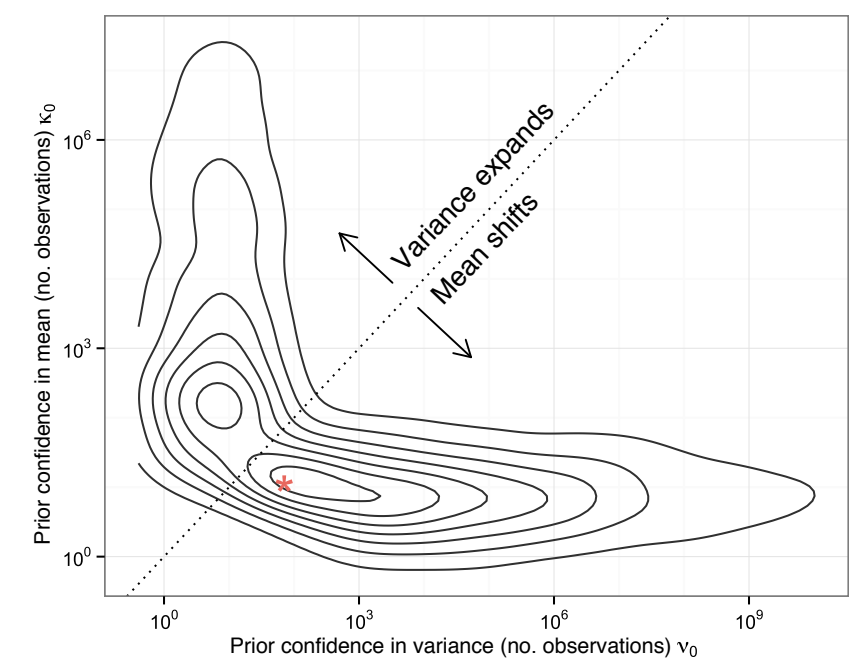

(a) Vroomen et al. (2007), recal. 64 exposures

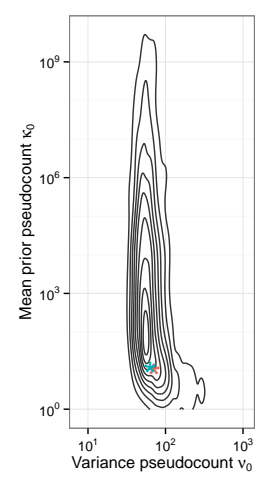

(b) V07, sel. ad., 64

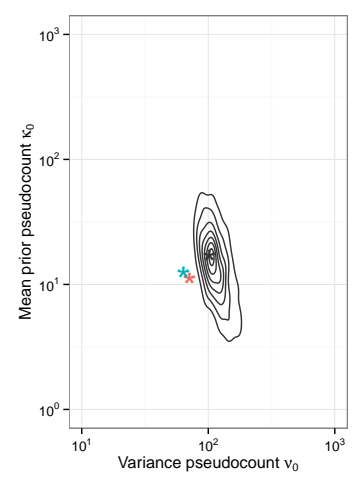

(c) V07, simult.

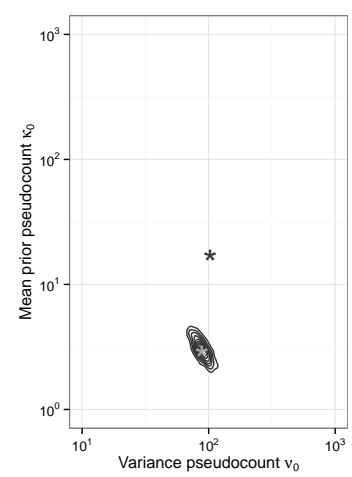

(d) MTurk data, simult.

Figure A.1: MCMC-estimated joint posterior density (contours) and MAPestimates (asterisks) of prior confidence parameters for all studies presented in the main text. Black asterisk shows MAP estimate for combined fit, and colored asterisks show earlier fits. Blue asterisk shows the MAP estimate for the selective adaptation condition, and red the recalibration condition. Panel (a): perceptual recalibration data from Vroomen et al. (2007) up 64 exposures (replotted for convenience). Panel (b): selective adaptation data from Vroomen et al. (2007) up to 64 exposures. Panel (c): data from both recalibration and selective adaptation Vroomen et al. (2007) up to 256 exposures. Panel (d): data from our web-based experiment. Light grey asterisk shows MAP estimate for these fits and dark asterisk shows MAP estimates from fit to Vroomen et al. (2007) data for comparison. 
the model to fit data which violates them.

All of these assumptions are assumptions of the model, and not the ideal adapter framework. In particular, the assumption that all observed cues (from one category) are identically distributed goes against the basic point of the ideal adapter analysis that cue distributions change from one situation to another, but it is a necessary simplification for specifically modeling beliefs about cues in the particular situation of a laboratory experiment. Also, a true ideal adapter would use their prior experience with category variance and base rate category probabilities to set these for each category, but we assume they are equal for $/ b /$ and $/ d$ / because this is a convenient simplification which reduces the number of hyperparameters that need to be estimate to fit the model without qualitatively changing the predictions. 
Table A.1: Assumptions of the belief updating model used to evaluate the ideal adapter framework predictions.

\begin{tabular}{|c|c|c|}
\hline Assumption & Simplification justified by data? & Problem? \\
\hline $\begin{array}{l}\text { Independently } \\
\text { and identically } \\
\text { distributed cues. }\end{array}$ & $\begin{array}{l}\text { No: non-stationarity/lack of invari- } \\
\text { ance means cue distributions are dif- } \\
\text { ferent. }\end{array}$ & $\begin{array}{l}\text { Not for modeling first block } \\
\text { adaptation in the lab (lis- } \\
\text { teners seem to assume that } \\
\text { they're in a totally new situ- } \\
\text { ation). }\end{array}$ \\
\hline $\begin{array}{l}\text { Equal prior vari- } \\
\text { ances }\end{array}$ & $\begin{array}{l}\text { Probably not, a priori. Also, pilot } \\
\text { simulations show that asymmetrical } \\
\text { prior variance leads to an overall in- } \\
\text { crease or decrease in the proportion } \\
\text { of /b/ responses regardless of the ex- } \\
\text { posure category, and the same pattern } \\
\text { shows up in the data. }\end{array}$ & $\begin{array}{l}\text { Not when using the after- } \\
\text { effect measure, which effec- } \\
\text { tively controls for changes } \\
\text { in overall rate of } / b / \text { re- } \\
\text { sponses. }\end{array}$ \\
\hline $\begin{array}{l}\text { Equal prior prob- } \\
\text { ability of /b/ vs. } \\
\text { /d/ }\end{array}$ & $\begin{array}{l}\text { No. /b/ is more than twice as likely } \\
\text { as /d/ in this context (Vitevitch \& } \\
\text { Luce, 2004). However, listeners make } \\
\text { roughly equal numbers of } / \mathrm{b} / \text { and } \\
\text { /d/ responses during pre-test so they } \\
\text { could infer that / b/ and /d/ are } \\
\text { equally likely in this task. }\end{array}$ & $\begin{array}{l}\text { No, based on pilot simula- } \\
\text { tions there's no qualitative } \\
\text { difference. }\end{array}$ \\
\hline $\begin{array}{l}\text { No change in be- } \\
\text { liefs about prior } \\
\text { probabilities }\end{array}$ & Unclear. & $\begin{array}{l}\text { No. When confidence in } \\
\text { prior probability is included } \\
\text { as a free hyperparameter, } \\
\text { it's always inferred to be } \\
\text { very high (no change). }\end{array}$ \\
\hline
\end{tabular}


Table A.1 - continued from previous page

\begin{tabular}{|c|c|c|}
\hline \multirow[b]{2}{*}{ Assumption } & \multirow{2}{*}{\multicolumn{2}{|c|}{ Cimnlifingtion inctifiod hud }} \\
\hline & & \\
\hline $\begin{array}{l}\text { Labeled data } \\
\text { (supervised adap- } \\
\text { tation) }\end{array}$ & $\begin{array}{l}\text { Yes. Listeners can classify the am- } \\
\text { biguous audio-visual stimuli nearly } \\
\text { perfectly }(98 \%) \text {, and they can't dis- } \\
\text { criminate between ambiguous and } \\
\text { prototypical audio-visual adaptor } \\
\text { from the same category ( } 52 \% \text { on an } \\
\text { ABX task; Vroomen, van Linden, } \\
\text { Keetels, et al., 2004). }\end{array}$ & \\
\hline $\begin{array}{l}\text { Only labeled data } \\
\text { counts for adapta- } \\
\text { tion }\end{array}$ & $\begin{array}{l}\text { Unclear. Listeners can adapt to } \\
\text { shifted distributions without addi- } \\
\text { tional information (C. M. Munson, } \\
\text { 2011). Fully optimal ideal adapter } \\
\text { predicts beliefs should be partially } \\
\text { updated (2.7), but depends on track- } \\
\text { ing the full posterior distribution for } \\
\text { all previous observations which may } \\
\text { be psychologically implausible. }\end{array}$ & $\begin{array}{l}\text { Probably not: doubling the } \\
\text { number of test trials doesn't } \\
\text { change adaptation. Other } \\
\text { category learning studies } \\
\text { suggest that when there are } \\
\text { many unlabeled training } \\
\text { items, they have little in- } \\
\text { fluence on later behavior } \\
\text { (Zhu et al., 2007), but it's a } \\
\text { question for future work. }\end{array}$ \\
\hline $\begin{array}{l}\text { Adaptation to in- } \\
\text { tegrated audio and } \\
\text { visual cues }\end{array}$ & Maybe, see main text for discussion. & $\begin{array}{l}\text { Probably not. There are } \\
\text { other reasons why the am- } \\
\text { biguous audio-visual adap- } \\
\text { tor might not be perceived } \\
\text { as ambiguous for the pur- } \\
\text { poses of adaptation as dis- } \\
\text { cussed above. }\end{array}$ \\
\hline $\begin{array}{l}\text { Normal dis- } \\
\text { tributions for } \\
\text { cues; Normal- } \\
\chi^{-2} \quad \text { parameter } \\
\text { distributions }\end{array}$ & $\begin{array}{l}\text { Normal cue distributions are a com- } \\
\text { mon assumption in computational } \\
\text { modeling, and using a conjugate prior } \\
\text { is a natural, convenient choice. }\end{array}$ & $\begin{array}{l}\text { No: any distribution that } \\
\text { is informationally efficient } \\
\text { (has few enough effec- } \\
\text { tive parameters) would } \\
\text { predict the same kind of } \\
\text { rapid/stable adaptation. }\end{array}$ \\
\hline
\end{tabular}


Table A.1 - continued from previous page

\begin{tabular}{|c|c|c|}
\hline Assumption & Simplification justified by data? & Problem? \\
\hline $\begin{array}{l}\text { Order of trials } \\
\text { doesn't matter } \\
\text { (exchangeability) }\end{array}$ & $\begin{array}{l}\text { No: Kraljic, Samuel, and Brennan } \\
\text { (2008) and carry over effects between } \\
\text { blocks observed in our own data and } \\
\text { Vroomen, van Linden, de Gelder, and } \\
\text { Bertelson (2007) (see Supplementary } \\
\text { Material for discussion). }\end{array}$ & $\begin{array}{l}\text { No: when modeling just } \\
\text { the first block of expo- } \\
\text { sure to simple cue statistics, } \\
\text { exchangeability is probably } \\
\text { reasonable. }\end{array}$ \\
\hline $\begin{array}{l}\text { Independence } \\
\text { of prior beliefs } \\
\text { about different } \\
\text { categories }\end{array}$ & $\begin{array}{l}\text { No. For instance: vowel F1 means are } \\
\text { all higher for female vs. male talk- } \\
\text { ers, which introduces positive corre- } \\
\text { lations between the means across talk- } \\
\text { ers (Hillenbrand et al., 1995). }\end{array}$ & $\begin{array}{l}\text { Probably not, especially } \\
\text { when prior beliefs are weak, } \\
\text { as we have argued is ex- } \\
\text { pected in laboratory studies } \\
\text { with unusual speech (and is } \\
\text { supported by the estimates } \\
\text { of weak prior confidence). }\end{array}$ \\
\hline
\end{tabular}

